\title{
NTP TECHNICAL REPORT ON THE TOXICITY STUDIES OF \\ O-CHLOROPYRIDINE (CASRN 109-09-1) ADMinistered DerMally AND IN DRINKING WATER TO F344/N RATS AND B6C3F1/N MICE
}

NTP TOX 83

FEBRUARY 2017 


\section{NTP Technical Report on the Toxicity Studies of o-Chloropyridine (CASRN 109-09-1) Administered Dermally and in Drinking Water to F344/N Rats and B6C3F1/N Mice}

Toxicity Report 83

February 2017

National Toxicology Program

Public Health Service

U.S. Department of Health and Human Services

ISSN: $2378-8992$

Research Triangle Park, North Carolina, USA 


\section{Foreword}

The National Toxicology Program (NTP) is an interagency program within the Public Health Service (PHS) of the Department of Health and Human Services (HHS) and is headquartered at the National Institute of Environmental Health Sciences of the National Institutes of Health (NIEHS/NIH). Three agencies contribute resources to the program: NIEHS/NIH, the National Institute for Occupational Safety and Health of the Centers for Disease Control and Prevention (NIOSH/CDC), and the National Center for Toxicological Research of the Food and Drug Administration (NCTR/FDA). Established in 1978, NTP is charged with coordinating toxicological testing activities, strengthening the science base in toxicology, developing and validating improved testing methods, and providing information about potentially toxic substances to health regulatory and research agencies, scientific and medical communities, and the public.

The Toxicity Study Report series began in 1991. The studies described in the Toxicity Study Report series are designed and conducted to characterize and evaluate the toxicologic potential of selected substances in laboratory animals (usually two species, rats and mice). Substances selected for NTP toxicity studies are chosen primarily on the basis of human exposure, level of production, and chemical structure. The interpretive conclusions presented in the Toxicity Study Reports are based only on the results of these NTP studies. Extrapolation of these results to other species, including characterization of hazards and risks to humans, requires analyses beyond the intent of these reports. Selection per se is not an indicator of a substance's toxic potential.

NTP conducts its studies in compliance with its laboratory health and safety guidelines and FDA Good Laboratory Practice Regulations and must meet or exceed all applicable federal, state, and local health and safety regulations. Animal care and use are in accordance with the Public Health Service Policy on Humane Care and Use of Animals. Studies are subjected to retrospective quality assurance audits before being presented for public review.

NTP Toxicity Study Reports are indexed in the National Center for Biotechnology Information (NCBI) Bookshelf and are available free of charge electronically on the NTP website (http://ntp.niehs.nih.gov). Additional information regarding this study may be requested through Central Data Management (CDM) at cdm@niehs.nih.gov. Toxicity data are available through NTP's Chemical Effects in Biological Systems (CEBS) database. 


\section{Table of Contents}

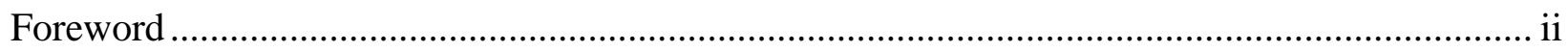

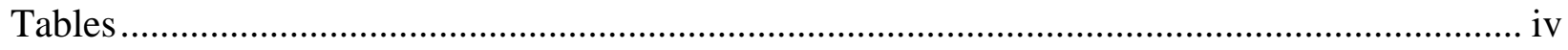

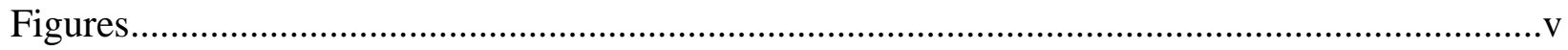

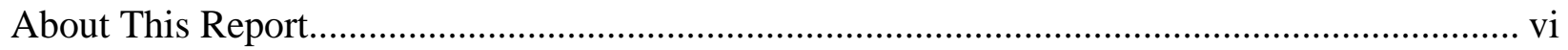

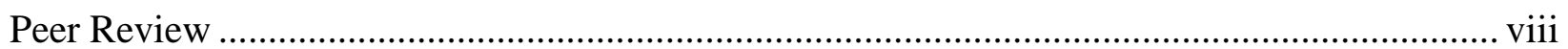

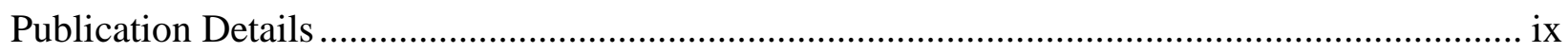

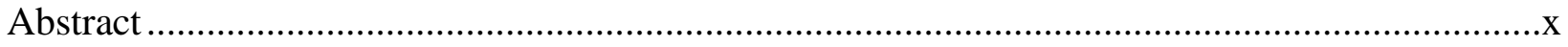

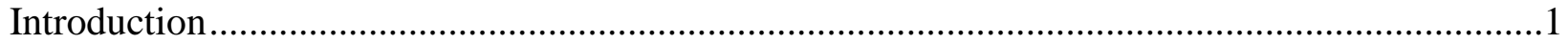

Chemical and Physical Properties ...................................................................................

Production, Use, and Human Exposure …………….......................................................

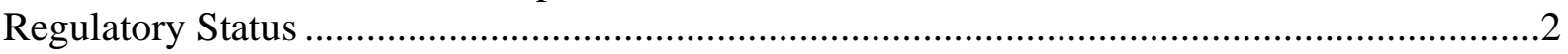

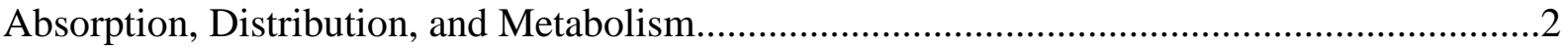

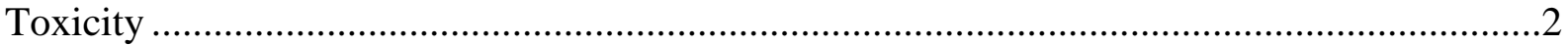

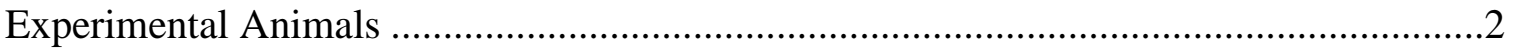

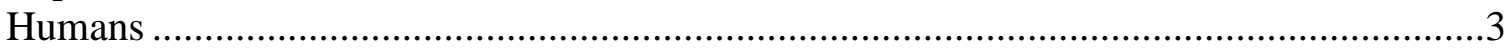

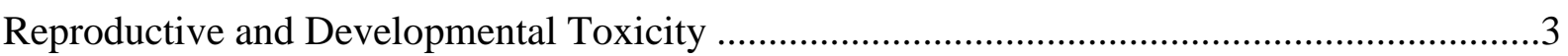

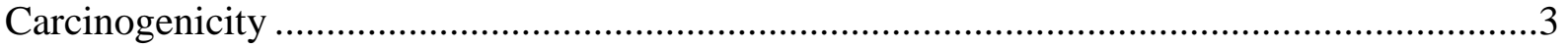

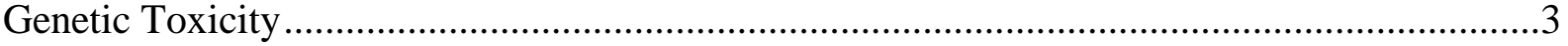

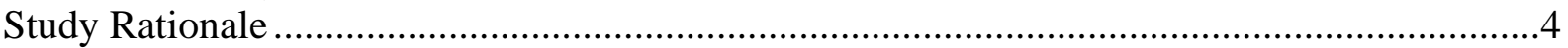

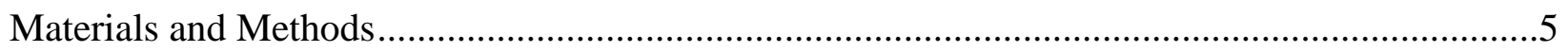

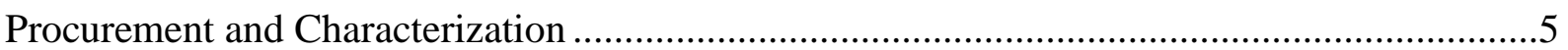

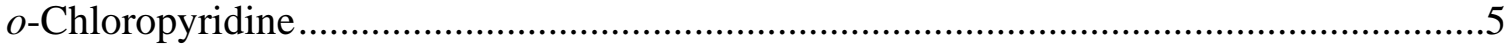

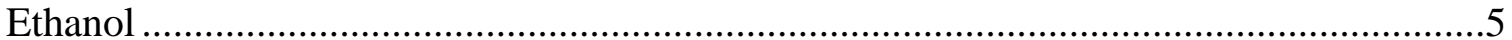

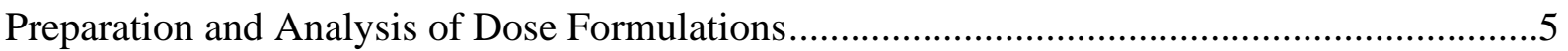

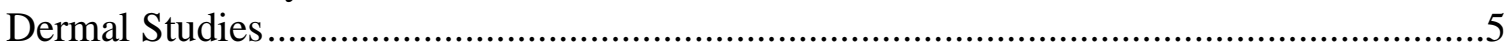

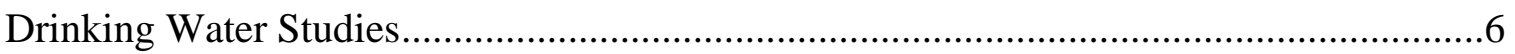

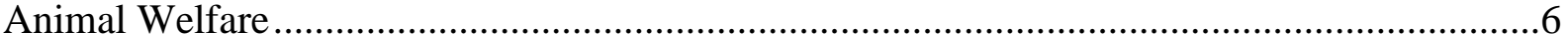

Two-week Dermal Studies .......................................................................................6

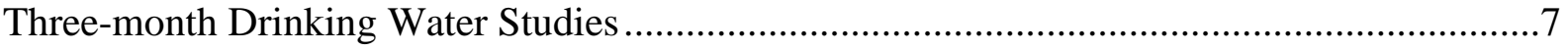

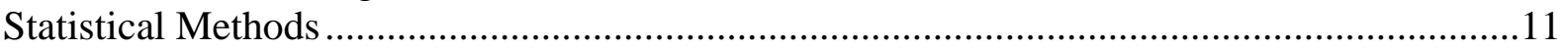

Calculation and Analysis of Lesion Incidences ..............................................................11

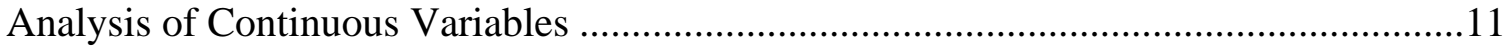

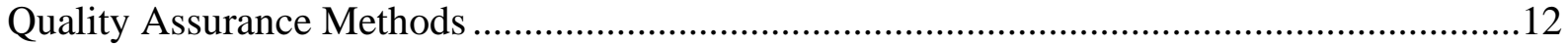

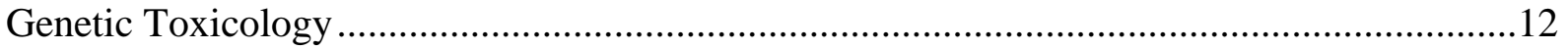

Salmonella typhimurium Mutagenicity Test Protocol ......................................................12

Mouse Peripheral Blood Micronucleus Test Protocol .........................................................13

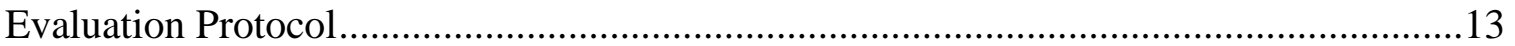

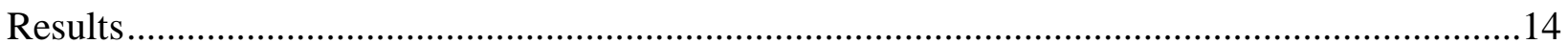

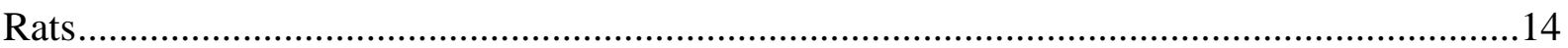

Two-week Dermal Study ………………………………........................................14 
Three-month Drinking Water Study …………………................................................15 Mice.

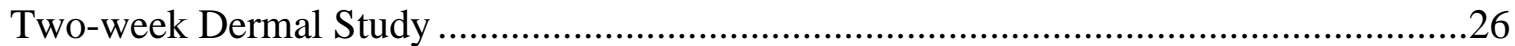

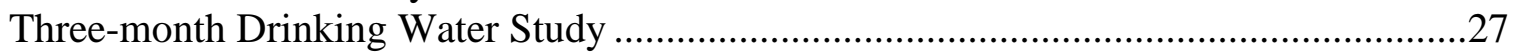

Absorption, Distribution, Metabolism, Excretion, and Toxicokinetics ......................................32

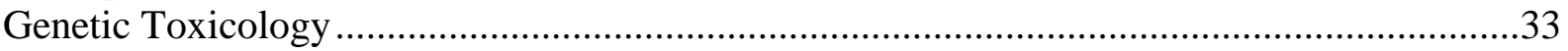

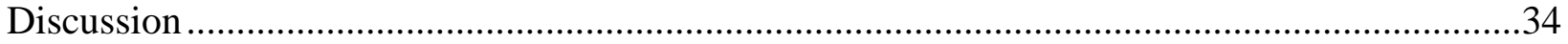

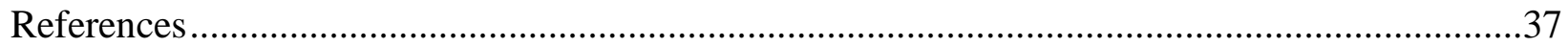

Appendix A. Summary of Lesions in Rats and Mice ………............................................ A-1

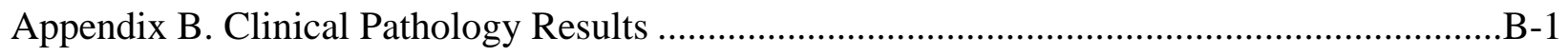

Appendix C. Organ Weights and Organ-Weight-to-Body-Weight Ratios ....................................-1

Appendix D. Reproductive Tissue Evaluations and Estrous Cycle Characterization ................ D-1

Appendix E. Genetic Toxicology …………………......................................................

Appendix F. Chemical Characterization and Dose Formulation Studies ……………...............F-1

Appendix G. Water and Compound Consumption in the Three-month Drinking Water

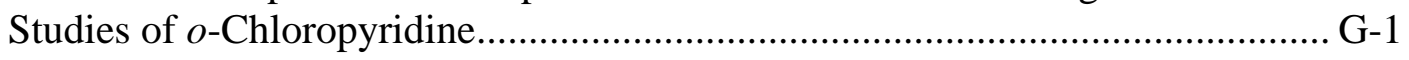

Appendix H. Ingredients, Nutrient Composition, and Contaminant Levels in NTP-2000 Rat and Mouse Ration.............................................................................. H-1

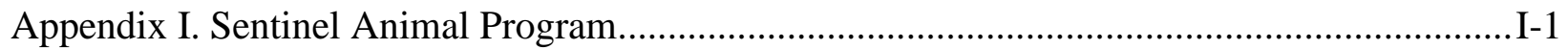

Appendix J. Absorption, Distribution, Metabolism, Excretion, and Toxicokinetics...................... J-1

\section{Tables}

Table 1. Experimental Design and Materials and Methods in the Studies of

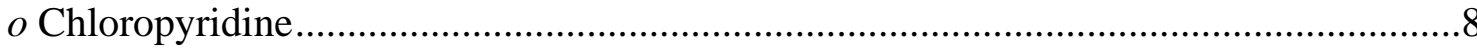

Table 2. Survival and Body Weights of Rats in the Two-week Dermal Study of $o$-Chloropyridine

Table 3. Liver Weights and Liver-Weight-to-Body-Weight Ratios for Male Rats in the Two-week Dermal Study of $o$-Chloropyridine.

Table 4. Survival, Body Weights, and Water Consumption of Rats in the Three-month Drinking Water Study of $o$-Chloropyridine

Table 5. Selected Clinical Pathology Data for Rats in the Three-month Drinking Water Study of $o$-Chloropyridine.

Table 6. Selected Organ Weights and Organ-Weight-to-Body-Weight Ratios for Rats in the Three-month Drinking Water Study of $o$-Chloropyridine

Table 7. Summary of Reproductive Tissue Evaluations for Male Rats in the Three-month Drinking Water Study of $o$-Chloropyridine

Table 8. Incidences of Selected Nonneoplastic Lesions in Rats in the Three-month Drinking Water Study of $o$-Chloropyridine

Table 9. Survival and Body Weights of Mice in the Two-week Dermal Study of $o$-Chloropyridine 
Table 10. Kidney Weights and Kidney-Weight-to-Body-Weight Ratios for Mice in the Two-week Dermal Study of $o$-Chloropyridine

Table 11. Survival, Body Weights, and Water Consumption of Mice in the Three-month Drinking Water Study of $o$-Chloropyridine

Table 12. Selected Hematology Data for Mice in the Three-month Drinking Water Study of $o$-Chloropyridine.

Table 13. Selected Organ Weights and Organ-Weight-to-Body-Weight Ratios for Mice in the Three-month Drinking Water Study of $o$-Chloropyridine

Table 14. Incidences of Hepatocyte Centrilobular Hypertrophy in the Liver of Mice in the Three-month Drinking Water Study of $o$-Chloropyridine

\section{Figures}

Figure 1. $o$-Chloropyridine (CASRN 109-09-1; Chemical Formula: $\mathrm{C}_{5} \mathrm{H}_{4} \mathrm{ClN}$; Molecular Weight: 113.55)

Figure 2. Growth Curves for Rats Exposed to $o$-Chloropyridine in Drinking Water for Three Months

Figure 3. Growth Curves for Mice Exposed to $o$-Chloropyridine in Drinking Water for Three Months

This report has been reformatted to meet new NTP publishing requirements; its content has not changed. 


\section{About This Report}

National Toxicology Program ${ }^{1}$

${ }^{1}$ Division of the National Toxicology Program, National Institute of Environmental Health

Sciences, Research Triangle Park, North Carolina, USA

\section{Collaborators}

G.K. Roberts; S.A. Elmore, N. Allison, B. Atkinson, P.E. Blackshear, C.R. Blystone, R.S. Chhabra, P.M. Foster, D.R. Germolec, M.J. Hooth, A.P. King-Herbert, G.E. Kissling, L.L. Lanning, D.E. Malarkey, B.S. McIntyre, S.D. Peddada, D. Ragland, G.B.J. Smith, S. Thakur, G.S. Travlos, M.K. Vallant, S. Waidyanatha, N.J. Walker, M.L. Wenk, K.L. Witt, G.W. Wolfe

Division of the National Toxicology Program, National Institute of Environmental Health Sciences, Research Triangle Park, North Carolina, USA

Evaluated and interpreted results and reported findings

G.K. Roberts, Ph.D., Study Scientist

S.A. Elmore, D.V.M., Study Pathologist

C.R. Blystone, Ph.D.

R.S. Chhabra, Ph.D.

P.M. Foster, Ph.D.

D.R. Germolec, Ph.D.

M.J. Hooth, Ph.D.

A.P. King-Herbert, D.V.M.

G.E. Kissling, Ph.D.

B.S. McIntyre, Ph.D.

D.E. Malarkey, D.V.M., Ph.D.

S.D. Peddada, Ph.D.

S. Thakur, Ph.D.

G.S. Travlos, D.V.M.

M.K. Vallant, M.S., MT

S. Waidyanatha, Ph.D.

N.J. Walker, Ph.D.

K.L. Witt, M.S.

BioReliance Corporation, Rockville, Maryland, USA

Conducted studies and evaluated pathology findings

M.L. Wenk, Ph.D., Principal Investigator

L.L. Lanning, D.V.M.

G.B.J. Smith, Ph.D.

D. Ragland, D.V.M.

Experimental Pathology Laboratories, Inc., Research Triangle Park, North Carolina, USA Conducted pathology review

N. Allison, D.V.M., Ph.D. 
ILS, Inc., Research Triangle Park, North Carolina, USA

Coordinated NTP Pathology Working Group (October 6, 2005)

P.E. Blackshear, D.V.M., Ph.D.

TherImmune Research Corporation, Gaithersburg, Maryland, USA

Provided SMVCE analysis

G.W. Wolfe, Ph.D., Principal Investigator

B. Atkinson, M.Sc.

\section{Contributors}

NTP Pathology Working Group, National Institute of Environmental Health Sciences, Research Triangle Park, North Carolina, USA

Participated in NTP Pathology Working Group (October 6, 2005)

R.A. Herbert, D.V.M., Ph.D., National Toxicology Program

G. Pearse, B.V.M.S., National Toxicology Program

Experimental Pathology Laboratories, Inc., Research Triangle Park, North Carolina, USA

Supervised pathology review

M.H. Hamlin, II, D.V.M., Principal Investigator

Dynamac Corporation, Research Triangle Park, North Carolina, USA

Prepared quality assessment audits

S. Brecher, Ph.D., Principal Investigator

S. Iyer, B.S.

V.S. Tharakan, D.V.M.

Social \& Scientific Systems, Inc., Research Triangle Park, North Carolina, USA

Provided statistical analyses

M.V. Smith, Ph.D., Principal Investigator

L.J. Betz, M.S.

S.F. Harris, B.S.

Biotechnical Services, Inc., Little Rock, Arkansas, USA

Prepared Toxicity Study Report

S.R. Gunnels, M.A., Principal Investigator

L.M. Harper, B.S.

J.I. Irving, M.A.P.

D.C. Serbus, Ph.D. 


\section{Peer Review}

The draft NTP Technical Report on the Toxicity Studies of o-Chloropyridine (CASRN 109-09-1) Administered Dermally and in Drinking Water to F344/N Rats and B6C3F1/N Mice was evaluated by the reviewers listed below. These reviewers served as independent scientists, not as representatives of any institution, company, or governmental agency. In this capacity, reviewers determined if the design and conditions of these NTP studies were appropriate and ensured that this NTP Toxicity Study Report presented the experimental results and conclusions fully and clearly.

\section{Peer Reviewers}

\section{William J. Brock, Ph.D.}

Brock Scientific Consulting, LLC

Montgomery Village, Maryland, USA

Laura A. Hansen, Ph.D.

Department of Biomedical Sciences

Creighton University School of Medicine

Omaha, Nebraska, USA

Sandra A. James-Yi, D.V.M., Ph.D.

Department of Biomedical Sciences and Pathobiology

Virginia-Maryland College of Veterinary Medicine

Virginia Tech

Blacksburg, Virginia, USA 


\section{Publication Details}

Publisher: National Toxicology Program

Publishing Location: Research Triangle Park, NC

ISSN: 2378-8992

DOI: https://doi.org/10.22427/NTP-TOX-83

Report Series: NTP Toxicity Report Series

Report Series Number: 83

Official citation: National Toxicology Program (NTP). 2017. NTP technical report on the toxicity studies of $o$-chloropyridine (CASRN 109-09-1) administered dermally and in drinking water to F344/N rats and B6C3F1/N mice. Research Triangle Park, NC: National Toxicology Program. Toxicity Report 83. 


\section{Abstract}

$o$-Chloropyridine is used as an intermediate in synthetic organic, pharmaceutical, and agricultural chemical (fungicides, herbicides) manufacture. It is also used as a catalyst for phase transfer and is a key intermediate in the manufacture of pyrithione-based biocides for use in cosmetics and various pharmaceutical products. $O$-Chloropyridine is available in purified (99\%), technical $(95 \%)$, or crude $(80 \%)$ grades. $o$-Chloropyridine was nominated for testing by NTP based on increasing production and use as a site-limited pharmaceutical and agrochemical intermediate, the potential for occupational and environmental exposures during its manufacture, its persistence in the environment (lasting longer than 6 months), evidence of mutagenicity based on results of several short-term test systems, and suspicion of carcinogenicity based on effects associated with structurally related chemicals. Male and female F344/N rats and B6C3F1/N mice received $o$-chloropyridine (99\% pure) dermally for 2 weeks or in drinking water for 3 months. Genetic toxicology studies were conducted in Salmonella typhimurium and mouse peripheral blood erythrocytes.

In the 2-week dermal studies, groups of five male and five female rats and mice were administered $o$-chloropyridine in ethanol 5 days per week over a 16-day period (12 dose days) at doses of $0,6.25,12.5,25,50$, or $100 \mathrm{mg} o$-chloropyridine/kg body weight. Vehicle control animals were administered ethanol alone. A constant concentration of test chemical per dose concentration was administered to each animal at volumes of $0.5 \mathrm{~mL} / \mathrm{kg}$ body weight for rats and $2 \mathrm{~mL} / \mathrm{kg}$ for mice. All dosed rats and mice survived to the end of the studies. The mean body weights of all dosed groups of rats and mice were similar to those of the vehicle control groups. Liver weights of 50 and $100 \mathrm{mg} / \mathrm{kg}$ male rats were significantly greater than those of the vehicle controls. No gross or microscopic lesions were considered related to $o$-chloropyridine administration.

In the 3-month toxicity studies, groups of 10 male and 10 female $\mathrm{F} 344 / \mathrm{N}$ rats and B6C3F1/N mice were exposed to $o$-chloropyridine in drinking water at concentrations of $0,10,30,100,300$, or 1,000 ppm (equal to average daily doses of approximately 1, 3, 9, 25, and $65 \mathrm{mg}$ $o$-chloropyridine $/ \mathrm{kg}$ body weight to male rats, $1,3,9,27$, and $70 \mathrm{mg} / \mathrm{kg}$ to female rats, $1.5,4.5$, 15,41 , and $110 \mathrm{mg} / \mathrm{kg}$ to male mice, and $1.3,4,12,38$, and $92 \mathrm{mg} / \mathrm{kg}$ to female mice) for 3 months. Additional groups of 10 male and 10 female rats designated for clinical pathology testing were exposed to the same concentrations for 22 days.

All rats survived to the end of the study. Mean body weights of male and female rats exposed to 1,000 ppm were significantly less than those of the controls. Water consumption by the $1,000 \mathrm{ppm}$ rats was less than that by the control groups during the first week of the study. In the $1,000 \mathrm{ppm}$ groups, thinness was noted in seven of 10 male rats and all female rats on day 8 , likely due to dehydration, and also in five of 10 male rats on day 64. The absolute and relative (except $100 \mathrm{ppm}$ ) right kidney weights of all exposed groups of male rats and of groups of female rats $(\geq 30 \mathrm{ppm})$ were greater than controls. Absolute and relative liver weights of male rats $(\geq 100 \mathrm{ppm})$ and female rats $(\geq 30 \mathrm{ppm})$ were significantly greater than those of the control groups. Epididymal sperm counts were significantly lower in male rats exposed to 1,000 ppm, indicating that $o$-chloropyridine exhibits the potential to be a reproductive toxicant. In the liver of male rats, the incidence of clear cell focus $(1,000 \mathrm{ppm})$ and the incidences and severities of hepatocyte cytoplasmic vacuolization $(\geq 300 \mathrm{ppm})$ were significantly higher. The incidence of hepatocyte cytoplasmic vacuolization was significantly greater in female rats also (1,000 ppm). 
There were also several low-magnitude histologic and hematologic responses in male and female rats suggesting an erythron effect characterized by a decreased erythron $(\geq 300 \mathrm{ppm}$ males and females) with a compensatory erythropoietic response: increased reticulocyte counts ( $\geq 300 \mathrm{ppm}$ males, $1,000 \mathrm{ppm}$ females) and hematopoietic cell proliferation in the spleen ( $\geq 300 \mathrm{ppm}$ males, $1,000 \mathrm{ppm}$ females). Splenic congestion (1,000 ppm males, $10 \mathrm{ppm}$ and $\geq 100 \mathrm{ppm}$ females) was also observed and may have been related to the erythron effect.

There were no treatment-related deaths in either sex of mice. The final mean body weight and mean body weight gain of $1,000 \mathrm{ppm}$ male mice were significantly less than those of the control group; the mean body weight gain of $300 \mathrm{ppm}$ female mice was significantly greater than that of the controls. Water consumption by the 1,000 ppm groups was less than that of the control groups during the first week of the study. The liver weights of all exposed groups of male mice and of 300 and 1,000 ppm female mice and the kidney weights of 1,000 ppm males (relative) and females (absolute and relative) were significantly greater than those of the controls. The incidences of hepatocyte centrilobular hypertrophy were significantly increased in the 300 and $1,000 \mathrm{ppm}$ groups of male and female mice, with exposure concentration-related increases in severity. An erythron effect, similar to that observed in rats, was observed in 1,000 ppm female mice only and was characterized by a small decrease in hematocrit value, hemoglobin concentration, and erythrocyte count; no accompanying erythropoietic response was observed in mice.

$o$-Chloropyridine was mutagenic in S. typhimurium strains TA98 and TA100 when tested with exogenous metabolic activation enzymes from rat or hamster liver; no mutagenic activity was observed in the absence of metabolic activation. In vivo, no increases in the frequencies of micronucleated erythrocytes were observed in peripheral blood of male or female mice exposed to $o$-chloropyridine for 3 months in drinking water.

Under the conditions of these 3-month drinking water studies, there were treatment-related organ weight changes and lesions in male and female rats and mice. The major target tissues in rats affected by $o$-chloropyridine exposure included the kidney, spleen, bone marrow, and liver; the major target organ in mice was the liver. The measurement most sensitive to $o$-chloropyridine exposure in male rats was increased absolute (all exposure groups) and relative (all exposure groups except $100 \mathrm{ppm}$ ) kidney weights in the absence of histopathologic changes [lowestobserved-effect level $($ LOEL $)=10 \mathrm{ppm}]$. In female rats, a LOEL of $10 \mathrm{ppm}$ was based on splenic congestion, observed in all treated groups (except $30 \mathrm{ppm}$ ), with hematopoietic cell proliferation and pigmentation in the spleen, bone marrow hyperplasia, and hematological changes at higher exposure concentrations. This pattern of erythropoietic responses in the spleen and bone marrow and hematologic changes was also observed in male rats at $300 \mathrm{ppm}$ or greater doses. In male mice, absolute and relative liver weights were significantly higher than controls in all treated groups (LOEL $10 \mathrm{ppm}$ ), with histologic changes (centrilobular hepatocyte hypertrophy) occurring at $300 \mathrm{ppm}$ and 1,000 ppm. In female mice, absolute and relative liver weights were significantly higher than controls, with increased incidences of centrilobular hepatocyte hypertrophy occurring at similar exposure concentrations (LOEL 300 ppm).

Synonyms: Alpha-chloropyridine; 2-chloro-(9Cl); 2-chloropyridine (8Cl); pyridines 
Summary of Findings Considered to be Toxicologically Relevant in Rats and Mice Exposed to oChloropyridine in Drinking Water for Three Months

\begin{tabular}{|c|c|c|c|c|}
\hline & $\begin{array}{c}\text { Male } \\
\text { F344/N Rats }\end{array}$ & $\begin{array}{c}\text { Female } \\
\text { F344/N Rats }\end{array}$ & $\begin{array}{c}\text { Male } \\
\text { B6C3F1/N Mice }\end{array}$ & $\begin{array}{c}\text { Female } \\
\text { B6C3F1/N Mice }\end{array}$ \\
\hline $\begin{array}{l}\text { Concentrations } \\
\text { in drinking } \\
\text { water }\end{array}$ & $\begin{array}{l}0,10,30,100,300 \text { or } \\
1,000 \mathrm{ppm}\end{array}$ & $\begin{array}{l}0,10,30,100,300 \text { or } \\
1,000 \mathrm{ppm}\end{array}$ & $\begin{array}{l}0,10,30,100,300 \\
\text { or } 1,000 \mathrm{ppm}\end{array}$ & $\begin{array}{l}0,10,30,100,300 \\
\text { or } 1,000 \mathrm{ppm}\end{array}$ \\
\hline $\begin{array}{l}\text { Average daily } \\
\text { doses }\end{array}$ & $0,1,3,9,25,65 \mathrm{mg} / \mathrm{kg}$ & $0,1,3,9,27,70 \mathrm{mg} / \mathrm{kg}$ & $\begin{array}{l}0,1.5,4.5,15,41 \\
110 \mathrm{mg} / \mathrm{kg}\end{array}$ & $\begin{array}{l}0,1.3,4,12,38 \\
92 \mathrm{mg} / \mathrm{kg}\end{array}$ \\
\hline Survival rates & $\begin{array}{l}10 / 10,10 / 10,10 / 10,10 / 10 \\
10 / 10,10 / 10\end{array}$ & $\begin{array}{l}10 / 10,10 / 10,10 / 10 \\
10 / 10,10 / 10,10 / 10\end{array}$ & $\begin{array}{l}10 / 10,10 / 10,10 / 10 \\
10 / 10,10 / 10,10 / 10\end{array}$ & $\begin{array}{l}9 / 10,10 / 10,10 / 10 \\
10 / 10,10 / 10,10 / 10\end{array}$ \\
\hline Body weights & $\begin{array}{l}\downarrow 16 \% \text { compared to } \\
\text { controls }(1,000 \mathrm{ppm})\end{array}$ & $\begin{array}{l}\downarrow 11 \% \text { compared to } \\
\text { controls }(1,000 \mathrm{ppm})\end{array}$ & $\begin{array}{l}\downarrow 19 \% \text { compared to } \\
\text { controls }(1,000 \mathrm{ppm})\end{array}$ & $\begin{array}{l}\uparrow 12 \% \text { compared to } \\
\text { controls }(300 \mathrm{ppm})\end{array}$ \\
\hline Clinical findings & $\begin{array}{l}\text { Thinness }(1,000 \text { ppm }) \text { in } \\
7 / 10 \text { on day } 8 \text { and } 5 / 10 \text { on } \\
\text { day } 64\end{array}$ & $\begin{array}{l}\text { Thinness }(1,000 \mathrm{ppm}) \text { in } \\
10 / 10 \text { on day } 8\end{array}$ & None & None \\
\hline Organ weights & $\begin{array}{l}\uparrow \text { Absolute and relative } \\
\text { kidney weights } \\
\uparrow \text { Absolute and relative } \\
\text { liver weights }\end{array}$ & $\begin{array}{l}\uparrow \text { Absolute and relative } \\
\text { kidney weights } \\
\uparrow \text { Absolute and relative } \\
\text { liver weights }\end{array}$ & $\begin{array}{l}\uparrow \text { Absolute and } \\
\text { relative liver } \\
\text { weights }\end{array}$ & $\begin{array}{l}\uparrow \text { Absolute and } \\
\text { relative liver } \\
\text { weights } \\
\uparrow \text { Absolute and } \\
\text { relative kidney } \\
\text { weights }\end{array}$ \\
\hline $\begin{array}{l}\text { Clinical } \\
\text { pathology } \\
\text { (week 14) }\end{array}$ & $\begin{array}{l}\downarrow \text { Erythrocyte count } \\
\downarrow \text { Hematocrit } \\
\downarrow \text { Hemoglobin } \\
\uparrow \text { Reticulocyte count }\end{array}$ & $\begin{array}{l}\downarrow \text { Erythrocyte count } \\
\downarrow \text { Hematocrit } \\
\downarrow \text { Hemoglobin } \\
\uparrow \text { Reticulocyte count }\end{array}$ & None & $\begin{array}{l}\downarrow \text { Erythrocyte count } \\
\downarrow \text { Hematocrit } \\
\downarrow \text { Hemoglobin }\end{array}$ \\
\hline $\begin{array}{l}\text { Reproductive } \\
\text { findings }\end{array}$ & $\downarrow$ Epididymal sperm count & None & None & None \\
\hline $\begin{array}{l}\text { Nonneoplastic } \\
\text { effects }\end{array}$ & $\begin{array}{l}\text { Liver: clear cell focus } \\
(0 / 10,0 / 10,0 / 10,0 / 10, \\
0 / 10,6 / 10) ; \text { hepatocyte } \\
\text { cytoplasmic vacuolization } \\
(0 / 10,0 / 10,0 / 10,0 / 10, \\
6 / 10,9 / 10) \\
\text { Spleen: hematopoietic cell } \\
\text { proliferation }(4 / 10,8 / 10, \\
6 / 10,6 / 10,10 / 10,10 / 10) \\
\text { congestion }(4 / 10,1 / 10, \\
\text { 2/10, 0/10, 7/10, 10/10) } \\
\text { Bone marrow: hyperplasia } \\
(1 / 10,0 / 10,2 / 10,1 / 10, \\
9 / 10,10 / 10)\end{array}$ & $\begin{array}{l}\text { Liver: hepatocyte } \\
\text { cytoplasmic } \\
\text { vacuolization }(0 / 10, \\
0 / 10,0 / 10,0 / 10,0 / 10, \\
9 / 10) \\
\text { Spleen: hematopoietic } \\
\text { cell proliferation }(3 / 10, \\
\text { 3/10, 6/10, 3/10, } 7 / 10, \\
\text { 10/10); congestion }(1 / 10, \\
7 / 10,5 / 10,6 / 10,6 / 10, \\
\text { 10/10) } \\
\text { Bone marrow: } \\
\text { hyperplasia }(0 / 10,0 / 10, \\
0 / 10,0 / 10,3 / 10,9 / 10)\end{array}$ & $\begin{array}{l}\text { Liver: centrilobular } \\
\text { hepatocyte } \\
\text { hypertrophy }(0 / 10 \\
0 / 10,0 / 10,1 / 10 \\
6 / 10,9 / 10)\end{array}$ & $\begin{array}{l}\text { Liver: centrilobular } \\
\text { hepatocyte } \\
\text { hypertrophy }(0 / 10, \\
0 / 10,0 / 10,0 / 10 \\
4 / 10,10 / 10)\end{array}$ \\
\hline
\end{tabular}

\section{Genetic toxicology}

Bacterial gene mutations:

Positive in Salmonella typhimurium strains TA98 and TA100 in the presence of rat or hamster liver S9 activation enzymes; negative in the absence of S9

Micronucleated erythrocytes

Mouse peripheral blood in vivo:

Negative in males and females 


\section{Introduction}

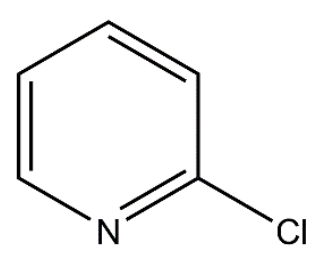

Figure 1. o-Chloropyridine (CASRN 109-09-1; Chemical Formula: $\mathrm{C}_{5} \mathrm{H}_{4} \mathrm{CIN}$; Molecular Weight: 113.55)

Synonyms: Alpha-chloropyridine; 2-chloro-(9Cl); 2-chloropyridine (8Cl); pyridines.

\section{Chemical and Physical Properties}

$o$-Chloropyridine is a colorless, oily liquid with a density of $1.205 \mathrm{~g} / \mathrm{cm}^{3}$ at $15^{\circ} \mathrm{C}^{1 ; 2}$ and a boiling point of $170^{\circ} \mathrm{C}^{1}$. The water solubility of $o$-chloropyridine is $2.5 \mathrm{~g} / 100 \mathrm{~g}$ at $25^{\circ} \mathrm{C}$; $o$-chloropyridine is soluble in alcohol and ether ${ }^{2 ; 3} . o$-Chloropyridine poses a slight fire hazard, and when exposed to heat can release toxic phosgene gas ${ }^{1 ; 4}$.

\section{Production, Use, and Human Exposure}

$o$-Chloropyridine can be prepared by direct chlorination of pyridine in the vapor phase $\left(>300^{\circ} \mathrm{C}\right)$ in the presence of a diluent ${ }^{5}$. One patented process (Reilly Industries, Inc.) involves selective chlorination of pyridine with chlorine in nitrogen in the presence of water vapor; synthesis occurs in two stages at $470^{\circ}$ and $290^{\circ} \mathrm{C}^{6}$. o-Chloropyridine is prepared for use as an intermediate by heating potassium pyrrole with chloroform. Another patented process involves the reaction of alpha-picoline with chlorine in the gas phase in the presence of water and a catalyst, such as pyrophyllite; this process yields a mix of chlorinated pyridines ${ }^{7}$. A third patented process reacts 2-hydroxypyridine with phosgene in the presence of an amide, such as N,N-dimethylformamide ${ }^{8}$.

$o$-Chloropyridine is listed as a chemical in commerce in the United States; no production or sales quantities were listed to avoid disclosure of the single producer's operations ${ }^{9}$. Companies are required to report production of 10,000 pounds or more or sales of $\$ 10,000$ or more. The United States Environmental Protection Agency ${ }^{10}$ lists $o$-chloropyridine in its Toxic Substances Control Act inventory. In 2006, the production and import of $o$-chloropyridine in the United States was reported to range from 1 million to 10 million pounds ${ }^{11}$.

$o$-Chloropyridine is used as an intermediate in synthetic organic, pharmaceutical, and agricultural chemical (fungicides, herbicides) manufacturing. It is also used as a catalyst for phase transfer ${ }^{1 ; 12}$. It is a key intermediate in the manufacture of pyrithione-based biocides for use in cosmetics and various pharmaceutical products ${ }^{13}$. $o$-Chloropyridine is used as a starting material in the production of the antihistamine, pheniramine, and the antiarrhythmic, diisopyramide 5 -Chloropyridine is available in purified (99\%), technical $(95 \%)$, or crude $(80 \%)$ grades $^{3 ; 12 ; 14-16}$.

There is potential for occupational exposure to $o$-chloropyridine during its production and use as an industrial chemical intermediate. A survey of the Olin Corporation plant in Rochester, NY, noted that significant exposures to chemicals in the $o$-chloropyridine processing area existed and 
that high vapor concentrations resulting from liquid spills and minor leaks were detected in the closed processing area, and quarterly sampling of personnel to detect exposures was recommended $^{17} . o$-Chloropyridine is not listed in the National Occupational Exposure Survey ${ }^{18}$.

Gehring et al. ${ }^{19}$ noted that the solubility of $o$-chloropyridine in organic solvents suggests that it might have high dermal absorption and subsequently, Gehring ${ }^{4}$ reported experimental evidence that chlorinated pyridines are rapidly absorbed through intact skin.

$o$-Chloropyridine has not been reported to occur naturally; however, it is reported to be an environmental contaminant. The Dow Chemical Company has identified it as a trace organic chemical in process streams and wastewater ${ }^{20}$. It has also been identified as a Rhine River pollutant in the Netherlands $(0.023 \mu \mathrm{g} / \mathrm{L})$ and a trace organic contaminant (detected, not quantified) in drinking water derived from river water in Barcelona, Spain ${ }^{21 ; 22}$.

$o$-Chloropyridine was detected as an intermediate product in amended freshwater sediment slurries; it arose from the biotransformation of 2,3-dichloropyridine under anaerobic (methanogenic) conditions. It was reported to be persistent and not to be further metabolized during a 6-month incubation period ${ }^{23}$. Adrian and Suflita ${ }^{24}$ reported that $o$-chloropyridine resisted biodegradation in anoxic aquifer slurries incubated for 11 months.

\section{Regulatory Status}

No standards or guidelines have been set by the National Institute for Occupational Safety and Health or the Occupational Safety and Health Administration for occupational exposure or workplace maximum allowable levels of $o$-chloropyridine. The American Conference of Governmental Industrial Hygienists has not recommended a threshold limit value or biological exposure index for this compound ${ }^{25} . o$-Chloropyridine is classified as a poisonous material by the U.S. Department of Transportation ${ }^{26}$. The USEPA ${ }^{17}$ has issued a requirement for health and safety data reporting on pyridine and pyridine derivatives, including $o$-chloropyridine.

\section{Absorption, Distribution, and Metabolism}

Incubation of $o$-chloropyridine with liver homogenate and cofactors yielded $o$-chloropyridine-Noxide and pyridine-N-oxide ${ }^{27}$. No disposition or metabolism studies of $o$-chloropyridine in experimental animals or humans could be found in the literature.

\section{Toxicity}

\section{Experimental Animals}

The acute toxicity of $o$-chloropyridine has been studied in mice, rats, and rabbits ${ }^{19}$. The mouse oral LD50 is $100 \mathrm{mg} / \mathrm{kg}$, and the intraperitoneal LD50 is $130 \mathrm{mg} / \mathrm{kg}$. Gross lesions included swollen and fatty livers as well as hemorrhage and necrosis at higher doses and swollen, edematous kidneys in some animals. Concurrent administration of methionine had a protective effect against toxicity, while cysteine and nicotinamide enhanced the toxicity of $o$-chloropyridine. In rabbits, $o$-chloropyridine was essentially as toxic when applied to the skin as when given by intraperitoneal injection. The rabbit dermal LD50 is $64 \mathrm{mg} / \mathrm{kg}$ and the intraperitoneal LD50 is $48 \mathrm{mg} / \mathrm{kg}$. The primary gross lesion, regardless of route of administration, was hemorrhagic necrosis of the liver. Instillation of undiluted or 
$10 \%$ o-chloropyridine solution in propylene glycol in the eyes of rabbits caused severe inflammation of the conjunctiva and moderate clouding of the cornea that persisted for 48 hours.

\section{Humans}

$o$-Chloropyridine was reported to be a skin irritant and toxic by ingestion ${ }^{1 ; 14}$.

\section{Reproductive and Developmental Toxicity}

No reproductive or developmental toxicity studies of $o$-chloropyridine in animals or humans were identified in the literature.

\section{Carcinogenicity}

No 2-year carcinogenicity studies of $o$-chloropyridine in animals or epidemiological studies or case reports investigating the association of exposure to $o$-chloropyridine and cancer risk in humans were identified in the literature.

\section{Genetic Toxicity}

$o$-Chloropyridine was mutagenic in Salmonella typhimurium strains TA97, TA98, TA100, and TA102 in tests conducted with exogenous metabolic activation (S9 liver enzymes), but no mutagenicity was detected in any of the strains without activation ${ }^{27 ; 28}$. Zimmerman et al. ${ }^{29}$ reported weak induction of aneuploidy in the yeast Saccharomyces cerevisiae treated with $o$-chloropyridine concentrations up to $0.50 \%$.

Tests in mammalian cells showed no induction of chromosomal aberrations by $o$-chloropyridine in African green monkey kidney cells (V3) in the absence of S9 enzymes ${ }^{30}$. However, when $\mathrm{V} 3$ cells were treated with $1,600 \mu \mathrm{g} / \mathrm{mL} o$-chloropyridine in the presence of pyridine $\mathrm{N}$-oxide, $25 \%$ of the cells were chromosomally aberrant; pyridine $\mathrm{N}$-oxide $(200 \mu \mathrm{g} / \mathrm{mL})$ alone was not clastogenic in V3 cells ${ }^{30}$.

In contrast to the results in $\mathrm{V} 3$ cells, $o$-chloropyridine was reported to induce gene mutations, chromosomal aberrations, and micronuclei in L5178Y mouse lymphoma cells with and without metabolic activation (rat liver S9 enzymes); the gene mutation and chromosomal aberration responses were stronger in the presence of $S 9^{31}$. These results led Dearfield et al. ${ }^{31}$ to postulate that substitution at the $o$-position promotes genotoxic effects of the halogenated pyridines through $\mathrm{N}$-oxidation by microsomal enzymes. This mechanism was also explored in experiments conducted by Chłopkiewicz et al. ${ }^{27}$ and designed to investigate the effects of $\mathrm{N}$-oxidation and $\mathrm{OH}$ radicals on the mutagenicity of $o$-chloropyridine in S. typhimurium TA100; they reported mutagenicity in a plate incorporation method in TA100 only with S9 and no, or greatly reduced, mutagenicity when glutathione, thiourea, D-mannitol, or pyridine N-oxide was added to the test mixture.

The metabolite $o$-chloropyridine N-oxide was not mutagenic in S. typhimurium strain TA100, with or without $\mathrm{S} 9$, when tested using a plate incorporation protocol ${ }^{27}$.

The structurally related compound, 3-chloropyridine, was not mutagenic in S. typhimurium strains TA97, TA98, TA100, or TA102, with or without S9 metabolic activation ${ }^{28 ; 32}$. 3-Chloropyridine induced a dose-related increase in chromosomal aberrations in V3 cells in the 
absence of S9 $9^{30}$; clastogenicity was eliminated when cultures were incubated in the presence of pyridine $\mathrm{N}$-oxide. 3-Chloropyridine induced small increases in gene mutations and chromosomal aberrations in mouse lymphoma L5178Y cells ${ }^{31}$, with and without S9 metabolic activation; micronuclei were induced by 3-chloropyridine only in the absence of S9.

\section{Study Rationale}

$o$-Chloropyridine was nominated for testing by NTP based on increasing production and use as a site-limited pharmaceutical and agrochemical intermediate, the potential for occupational and environmental exposures during its manufacture, its persistence in the environment (lasting longer than 6 months), evidence of mutagenicity based on results of several short-term test systems, and suspicion of carcinogenicity based on structure and evidence of mutagenic or carcinogenic effects associated with structurally related chemicals. Dermal exposure was selected for the 2-week studies based on the likely route of occupational exposure. The route of exposure was changed to oral, via drinking water, in the 3-month studies due to concerns regarding skin irritation following longer dermal exposure and to allow comparison of $o$-chloropyridine toxicity with the results of the NTP 3-month drinking water studies of a structurally similar compound, pyridine ${ }^{33}$. 


\section{Materials and Methods}

\section{Procurement and Characterization}

\section{o-Chloropyridine}

$o$-Chloropyridine was obtained from Aldrich Chemical Company (Milwaukee, WI) in one lot $(15306 \mathrm{CN})$ that was used in the 2-week dermal and 3-month drinking water studies. Identity and purity analyses were conducted by the analytical chemistry laboratory at Battelle (Columbus, $\mathrm{OH}$, and Richland, WA), Chemir/Polytech Laboratories, Inc. (Maryland Heights, MO), Galbraith Laboratories, Inc. (Knoxville, TN), and the study laboratory, BioReliance Corporation (Rockville, MD) (Appendix F). Reports on analyses performed in support of the $o$-chloropyridine studies are on file at the National Institute of Environmental Health Sciences.

The chemical, a clear colorless liquid, was identified as $o$-chloropyridine by infrared (IR) and proton and carbon-13 nuclear magnetic resonance spectroscopy. The purity of lot 15306CN was determined by elemental analyses and two gas chromatography (GC) systems. Elemental analyses for carbon, hydrogen, nitrogen, and chlorine were in agreement with the theoretical values for $o$-chloropyridine. GC by both systems indicated one major peak and two impurities with areas at least $0.1 \%$ relative to the major peak area. The overall purity of lot $15306 \mathrm{CN}$ was determined to be $99 \%$ or greater.

To ensure stability, the bulk chemical was stored at room temperature in sealed amber glass bottles under a headspace of inert gas, protected from light and moisture. Periodic reanalyses of the bulk chemical were performed during the 2-week and 3-month studies using GC, and no degradation of the bulk chemical was detected.

\section{Ethanol}

95\% Ethanol was obtained from Clear Spring Distilling Company (Clearwater, KY) in one lot (21049312) that was used as the dosing vehicle in the 2-week dermal studies. Lot 21049312, a clear liquid, was identified as ethanol by IR spectroscopy. The purity of lot 21049312 was determined using GC. Analysis indicated one major peak and three impurities each with a relative concentration of less than or equal to $0.0001 \%$.

\section{Preparation and Analysis of Dose Formulations}

\section{Dermal Studies}

The dose formulations were prepared by mixing $o$-chloropyridine with $95 \%$ ethanol (Table F-2). Stability studies of a $3.125 \mathrm{mg} / \mathrm{mL}$ dose formulation were performed by the analytical chemistry laboratory with GC. Stability was confirmed for at least 43 days for dose formulations stored in sealed amber glass vials at room temperature, $5^{\circ} \mathrm{C}$, or $-20^{\circ} \mathrm{C}$. Data from a simulated animal room stability study indicated that $o$-chloropyridine is stable dissolved in $95 \%$ ethanol when exposed to light for up to 3 hours at room temperature. The dose formulations were stored under a headspace of inert gas in refrigerated amber glass vials with Teflon ${ }^{\circledR}$-lined lids for up to 23 days. 
The dose formulations were analyzed on the day they were prepared by the study laboratory using GC. All seven dose formulations analyzed for rats and mice were within $10 \%$ of the target concentrations. Animal room samples of these dose formulations were also analyzed; one of five formulations for rats and all five formulations for mice were within $10 \%$ of the target concentrations. High concentrations measured for some of the rat animal room samples were attributed to improper sealing of the vials after dosing and possible solvent evaporation.

\section{Drinking Water Studies}

The dose formulations were prepared by mixing $o$-chloropyridine with tap water (Table F-2). Stability studies of the $10 \mathrm{ppm}$ dose formulation were performed by the analytical chemistry laboratory with high-performance liquid chromatography (HPLC). Stability was confirmed for at least 43 days for dose formulations stored in sealed polyethylene bottles protected from light at $5^{\circ} \mathrm{C}$ and for at least 8 days under simulated animal room conditions. The dose formulations were stored refrigerated in Cubitainers ${ }^{\circledR}$ with taps, protected from light, for up to 21 days.

The dose formulations were analyzed at the beginning, midpoint, and end of the studies by the study laboratory using HPLC; animal room samples of these dose formulations were also analyzed. All 15 dose formulations analyzed and used for rats and mice were within $10 \%$ of the target concentrations. Of the animal room samples analyzed, all 15 for rats and 12 of 15 for mice were within $10 \%$ of the target concentrations, and the remaining three were less than $12 \%$ of the target concentration.

\section{Animal Welfare}

Animal care and use are in accordance with the Public Health Service Policy on Humane Care and Use of Animals. All animal studies were conducted in an animal facility accredited by the Association for the Assessment and Accreditation of Laboratory Animal Care International. Studies were approved by the BioReliance Corporation Animal Care and Use Committee and conducted in accordance with all relevant NIH and NTP animal care and use policies and applicable federal, state, and local regulations and guidelines.

\section{Two-week Dermal Studies}

Male and female F344/N rats and B6C3F1/N mice were obtained from the NTP colony maintained at Taconic Farms, Inc. (Germantown, NY). On receipt, the rats and mice were 4 weeks old. Animals were quarantined for 12 (rats) or 13 (mice) days and were 5 to 6 weeks old on the first day of the studies. $o$-Chloropyridine in $95 \%$ ethanol was applied using automatic (adjustable volume) pipettes to the center of a shaved area of skin on the dorsal surface from just posterior to the scapulae to the base of the tail; the shaved area was larger than the application site. A constant concentration of test chemical per dose concentration was administered to each animal at volumes of $0.5 \mathrm{~mL} / \mathrm{kg}$ body weight for rats and $2 \mathrm{~mL} / \mathrm{kg}$ for mice. Five male and five female rats and mice per dose group were administered $o$-chloropyridine in ethanol 5 days per week over a 16-day period (12 dose days). Rats and mice were administered 0, 6.25, 12.5, 25, 50 , or $100 \mathrm{mg} o$-chloropyridine/kg body weight. Vehicle control animals were administered ethanol alone. Feed and water were available ad libitum. Rats and mice were housed individually. Clinical findings were recorded daily. The animals were weighed initially, on day 8 , and at the end of the studies. Prior to dosing and at the start of the studies, five male and five 
female rats and mice were selected for parasite evaluation and gross observation for disease presence. Details of the study design and animal maintenance are summarized in Table 1.

Necropsies were performed on all rats and mice. The heart, right kidney, liver, lung, right testis, and thymus were weighed. Histopathologic examinations were performed on gross lesions in rats; no gross lesions were observed in mice.

\section{Three-month Drinking Water Studies}

For the 3-month studies, the route of exposure was changed from dermal administration to drinking water, allowing for comparison of $o$-chloropyridine toxicity with the results of the NTP 3 -month drinking water studies of a structurally similar compound, pyridine ${ }^{33}$. Male and female F344/N rats and B6C3F1/N mice were obtained from the NTP colony maintained at Taconic Farms, Inc. (Germantown, NY). On receipt, the rats and mice were 3 weeks old. Animals were quarantined for 11 to 12 days (rats) or 14 to 15 days (mice); rats were 5 to 6 weeks old and mice were 6 to 7 weeks old on the first day of the studies. Upon arrival at the facility, five male and five female rats and mice were randomly selected for parasite evaluation and gross observation for evidence of disease. Additionally, the health of the animals was monitored during the studies according to the protocols of the NTP Sentinel Animal Program (Appendix I). All test results were negative.

The core study animals consisted of groups of 10 male and 10 female rats and mice that were exposed to $o$-chloropyridine in drinking water at concentrations of $0,10,30,100,300$, or $1,000 \mathrm{ppm}$ for 14 weeks. Exposure concentrations for the 3-month drinking water studies were selected for comparison to the 3-month pyridine study results ${ }^{33}$. Additional groups of 10 male and 10 female rats designated as clinical pathology study rats were exposed to the same concentrations for 22 days for evaluation of clinical pathology endpoints at days 4 and 22; these animals were not assessed for other endpoints. Clinical pathology results presented for week 14 are from core study animals. Feed and water were available ad libitum. Rats and female mice were housed five per cage and male mice were housed individually. Core study animals were weighed and clinical findings were recorded initially, weekly, and the end of the studies. Water consumption by core study animals was recorded weekly by cage. Details of the study design and animal maintenance are summarized in Table 1.

Animals were anesthetized with a 70\% carbon dioxide:30\% oxygen mixture, and blood was collected from the retroorbital sinus of clinical pathology study rats on days 4 and 22 and from core study rats and mice at the end of the 3-month studies for hematology and clinical chemistry (rats only) analyses (Appendix B). Hematology and clinical chemistry parameters were measured using a ABX Pentra C+ Analyzer (Horiba Instruments Corporation, Irvine, CA) and a Hitachi 717 Analyzer (Boehringer Mannheim, Indianapolis, IN), respectively, using reagents supplied by the manufacturers. The parameters measured are listed in Table 1.

At the end of the 3-month drinking water studies, samples were collected for sperm motility and vaginal cytology evaluations on rats and mice exposed to $0,100,300$, or 1,000 ppm. The parameters evaluated are listed in Table 1. For 12 consecutive days prior to scheduled terminal sacrifice, the vaginal vaults of the females were moistened with saline, if necessary, and samples of vaginal fluid and cells were stained. Relative numbers of leukocytes, nucleated epithelial cells, and large squamous epithelial cells were determined and used to ascertain estrous cycle stage 
(i.e., diestrus, proestrus, estrus, and metestrus). Male animals were evaluated for sperm count and motility. The left testis and left epididymis were isolated and weighed. The tail of the epididymis (cauda epididymis) was then removed from the epididymal body (corpus epididymis) and weighed. Test yolk (rats) or modified Tyrode's buffer (mice) was applied to slides and a small incision was made at the distal border of the cauda epididymis. The sperm effluxing from the incision were dispersed in the buffer on the slides, and the numbers of motile and nonmotile spermatozoa were counted for five fields per slide by two observers. Following completion of sperm motility estimates, each left cauda epididymis was placed in buffered saline solution. Caudae were finely minced, and the tissue was incubated in the saline solution and then heat fixed at $65^{\circ} \mathrm{C}$. Sperm density was then determined microscopically with the aid of a hemacytometer. To quantify spermatogenesis, the testicular spermatid head count was determined by removing the tunica albuginea and homogenizing the left testis in phosphatebuffered saline containing 10\% dimethyl sulfoxide. Homogenization-resistant spermatid nuclei were counted with a hemacytometer.

Necropsies were performed on all core study rats and mice. The heart, right kidney, liver, lung, right testis, and thymus were weighed. Tissues for microscopic examination were fixed and preserved in 10\% neutral buffered formalin (eyes were first fixed in Davidson's solution), processed and trimmed, embedded in paraffin, sectioned to a thickness of 4 to $6 \mu \mathrm{m}$, and stained with hematoxylin and eosin. Complete histopathologic examinations were performed by the study laboratory pathologist on core study rats and mice in the 0 and 1,000 ppm groups; the bone marrow, kidney, liver, and spleen of rats and the kidney and liver of mice were examined in the remaining core study groups. If findings were observed in the 1,000 ppm group, a read down was performed in which lower exposure groups were examined until a no-effect level was determined for a given endpoint. Table 1 lists the tissues and organs routinely examined.

After a review of the laboratory reports and selected histopathology slides by a quality assessment (QA) pathologist, the findings and reviewed slides were submitted to a NTP Pathology Working Group (PWG) coordinator for a second independent review. Any inconsistencies in the diagnoses made by the laboratory and QA pathologists were resolved by the NTP pathology peer review process. Final diagnoses for reviewed lesions represent a consensus of the PWG or a consensus between the study laboratory pathologist, NTP pathologist, QA pathologist(s), and the PWG coordinator. Details of these review procedures have been described, in part, by Maronpot and Boorman ${ }^{34}$ and Boorman et al. ${ }^{35}$.

Table 1. Experimental Design and Materials and Methods in the Studies of $o$-Chloropyridine

\section{Two-week Dermal Studies Three-month Drinking Water Studies}

\section{Study Laboratory}

BioReliance Corp. (Rockville, MD)

Strain and Species

F344/N rats

B6C3F1/N mice

\section{Animal Source}

Taconic Farms, Inc. (Germantown, NY)
BioReliance Corp. (Rockville, MD)

F344/N rats

$\mathrm{B} 6 \mathrm{C} 3 \mathrm{~F} 1 / \mathrm{N}$ mice

Taconic Farms, Inc. (Germantown, NY) 


\section{Time Held Before Studies}

Rats: 12 days

Mice: 13 days

Average Age When Studies Began

5-6 weeks

\section{Date of First Dose or Exposure}

Rats: September 19, 2000

Mice: September 20, 2000

\section{Duration of Dosing or Exposure}

5 exposures per week for 16 days

\section{Date of Last Dose or Exposure}

Rats: October 4, 2000

Mice: October 5, 2000

\section{Necropsy Dates}

Rats: October 5, 2000

Mice: October 6, 2000

\section{Average Age at Necropsy}

8-9 weeks

\section{Size of Study Groups}

5 males and 5 females

\section{Method of Distribution}

Animals were distributed randomly into groups of approximately equal initial mean body weights.

\section{Animals per Cage}

1 animal

\section{Method of Animal Identification}

Tail tattoo

\section{Diet}

NTP-2000 open formula pelleted diet (Zeigler Brothers, Inc., Gardners, PA), available ad libitum, changed once weekly

\section{Water}

Tap water (Washington, D.C., Suburban Sanitary Commission Potomac Plant) via automatic watering system (Edstrom Industries, Inc., Waterford, WI), available ad libitum
Rats: 11 (males) or 12 (females) days

Mice: 14 (males) or 15 (females) days

Rats: 5-6 weeks

Mice: 6-7 weeks

Rats: July 28, 2003

Mice: July 31, 2003

14 weeks (core study)

Rats: October 27 (males) or 28 (females), 2003

Mice: October 30 (males) or 31 (females), 2003

Rats: October 27 (males) or 28 (females), 2003

Mice: October 30 (males) or 31 (females), 2003

Rats: $18-19$ weeks

Mice: 19-20 weeks

10 males and 10 females

Same as 2-week studies

Rats: 5

Mice: 1 (males) or 5 (females)

Tail tattoo

Same as 2-week studies

Same as 2-week studies, except via amber glass bottles equipped with stainless steel sipper tubes and neoprene stoppers with screw on polypropylene lids 


\section{Cages}

Polycarbonate (Lab Products, Inc., Seaford, DE), changed once weekly

\section{Bedding}

Irradiated, heat-treated Sani-Chips ${ }^{\circledR}$ (P.J. Murphy Forest Products Corp., Montville, NJ), changed once weekly

\section{Cage Filters}

Remay $^{\circledR} 2016$ (Snow Filtration, Cincinnati, OH)

\section{Racks}

Stainless steel (Lab Products, Inc., Seaford, DE), changed once every 2 weeks

\section{Animal Room Environment}

Temperature: $72^{\circ} \pm 3^{\circ} \mathrm{F}$

Relative humidity: $50 \% \pm 15 \%$

Room fluorescent light: 12 hours/day

Room air changes: 10/hour

\section{Doses or Exposure}

$0,6.25,12.5,25,50$, or $100 \mathrm{mg} / \mathrm{kg}$

\section{Type and Frequency of Observation}

Observed twice daily; animals were weighed initially, on day 8 , and at the end of the studies; clinical findings were recorded daily.

\section{Method of Sacrifice}

Carbon dioxide asphyxiation

\section{Necropsy}

Necropsies were performed on all animals. Organs weighed were heart, right kidney, liver, lung, right testis, and thymus.

\section{Clinical Pathology}

None
Same as 2-week studies; changed twice weekly for rats and female mice and once weekly for male mice; rotated every 2 weeks

Same as 2-week studies; changed twice weekly for rats and female mice and once weekly for male mice

Same as 2-week studies

Same as 2-week studies; changed and rotated once every 2 weeks

Same as 2-week studies

$0,10,30,100,300$, or $1,000 \mathrm{ppm}$
Observed twice daily; core study animals were weighed and clinical findings were recorded initially, weekly, and at the end of the studies. Water consumption by core study animals was recorded weekly by cage.

Same as 2-week studies

Necropsies were performed on all core study animals. Organs weighed were heart, right kidney, liver, lung, right testis, and thymus.

Blood was collected from the retroorbital sinus of clinical pathology study animals on days 4 and 22 and from core study animals at the end of the studies for hematology and clinical chemistry (rats only).

Hematology: hematocrit; hemoglobin; erythrocyte, reticulocyte, nucleated erythrocyte, and platelet counts; mean cell volume; mean cell hemoglobin; mean cell hemoglobin concentration; and leukocyte count and differentials.

Clinical chemistry: urea nitrogen, creatinine, total protein, albumin, alanine aminotransferase, alkaline phosphatase, creatine kinase, sorbitol dehydrogenase, and bile acids 


\section{Histopathology}

Gross lesions were examined.

Sperm Motility and Vaginal Cytology

None

\begin{abstract}
Complete histopathology was performed on 0 and $1,000 \mathrm{ppm}$ core study rats and mice and a read down was performed when needed. In addition to gross lesions and tissue masses, the following tissues were examined: adrenal gland, bone with marrow, brain, clitoral gland, esophagus, eye, gallbladder (mice), Harderian gland, heart and aorta, large intestine (cecum, colon, rectum), small intestine (duodenum, jejunum, ileum), kidney, liver, lung (with mainstem bronchus), lymph nodes (mandibular and mesenteric), mammary gland, nose, ovary, pancreas, parathyroid gland, pituitary gland, preputial gland, prostate gland, salivary gland, seminal vesicle, skin, spleen, stomach (forestomach and glandular), testis (with epididymis), thymus, thyroid gland, trachea, urinary bladder, and uterus. The bone marrow, kidney, liver, and spleen were examined in the remaining groups of core study rats, and the kidney and liver were examined in the remaining groups of mice.
\end{abstract}

\section{Statistical Methods}

\section{Calculation and Analysis of Lesion Incidences}

The incidences of lesions are presented in Appendix A as the numbers of animals bearing such lesions at a specific anatomic site and the numbers of animals with that site examined microscopically. The Fisher exact test ${ }^{36}$, a procedure based on the overall proportion of affected animals, was used to determine significance.

\section{Analysis of Continuous Variables}

Two approaches were employed to assess the significance of pairwise comparisons between dosed or exposed and control groups in the analysis of continuous variables. Organ and body weight data, which historically have approximately normal distributions, were analyzed with the parametric multiple comparison procedures of Dunnett ${ }^{37}$ and Williams ${ }^{38 ; 39}$. Hematology, clinical chemistry, spermatid, and epididymal spermatozoal data, which have typically skewed distributions, were analyzed using the nonparametric multiple comparison methods of Shirley ${ }^{40}$ (as modified by Williams ${ }^{41}$ ), and Dunn ${ }^{42}$. Jonckheere's test ${ }^{43}$ was used to assess the significance 
of the dose-related trends and to determine whether a trend-sensitive test (Williams' or Shirley's test) was more appropriate for pairwise comparisons than a test that does not assume a monotonic dose-related trend (Dunnett's or Dunn's test). Prior to statistical analysis, extreme values identified by the outlier test of Dixon and Massey ${ }^{44}$ were examined by NTP personnel, and implausible values were eliminated from the analysis. Proportions of regular cycling females in each exposed group were compared to the control group using the Fisher exact test ${ }^{36}$. Tests for extended periods of estrus, diestrus, metestrus, and proestrus, as well as skipped estrus and skipped diestrus were constructed based on a Markov chain model proposed by Girard and Sager ${ }^{45}$. For each exposure group, a transition probability matrix was estimated for transitions among the proestrus, estrus, metestrus, and diestrus stages, with provision for extended stays within each stage as well as for skipping estrus or diestrus within a cycle. Equality of transition matrices among exposure groups and between the control group and each exposed group was tested using chi-square statistics.

\section{Quality Assurance Methods}

The 2-week and 3-month studies were conducted in compliance with Food and Drug Administration Good Laboratory Practice Regulations ${ }^{46}$. In addition, as records from the 3-month studies were submitted to the NTP Archives, these studies were audited retrospectively by an independent QA contractor. Separate audits covered completeness and accuracy of the pathology data, pathology specimens, final pathology tables, and a draft of this NTP Toxicity Study Report. Audit procedures and findings are presented in the reports and are on file at NIEHS. The audit findings were reviewed and assessed by NTP staff, and all comments were resolved or otherwise addressed during the preparation of this Toxicity Study Report.

\section{Genetic Toxicology}

\section{Salmonella typhimurium Mutagenicity Test Protocol}

Testing was performed as reported by Zeiger et al. ${ }^{47} . o$-Chloropyridine was sent to the laboratory as a coded aliquot. It was incubated with the Salmonella typhimurium tester strains TA98 and TA100 either in buffer or S9 mix (metabolic activation enzymes and cofactors from Aroclor 1254-induced male Sprague-Dawley rat or Syrian hamster liver) for 20 minutes at $37^{\circ} \mathrm{C}$. Top agar supplemented with 1-histidine and d-biotin was added, and the contents of the tubes were mixed and poured onto the surfaces of minimal glucose agar plates. Histidine-independent mutant colonies arising on these plates were counted following incubation for 2 days at $37^{\circ} \mathrm{C}$.

Each trial consisted of triplicate plates of concurrent positive and negative controls and five doses of $o$-chloropyridine. The assay limit dose was $10,000 \mu \mathrm{g} /$ plate. All positive trials were repeated under the conditions that elicited the positive response.

In this assay, a positive response is defined as a reproducible, dose-related increase in histidineindependent (revertant) colonies in any one strain/activation combination. An equivocal response is defined as an increase in revertants that is not dose related, is not reproducible, or is not of sufficient magnitude to support a determination of mutagenicity. A negative response is obtained when no increase in revertant colonies is observed following chemical treatment. There is no minimum percentage or fold increase required for a chemical to be judged positive or weakly 
positive, although positive calls are typically reserved for increases in mutant colonies that are at least twofold over background.

\section{Mouse Peripheral Blood Micronucleus Test Protocol}

A detailed discussion of this assay is presented by MacGregor et al. ${ }^{48}$. At the end of the 3-month toxicity study, peripheral blood samples were obtained from male and female mice. Smears were immediately prepared and fixed in absolute methanol. The methanol-fixed slides were stained with acridine orange and coded. Slides were scanned to determine the frequency of micronuclei in 2,000 normochromatic (mature) erythrocytes (NCEs) in each of five animals per exposure group. In addition, the percentage of polychromatic erythrocytes (PCEs; reticulocytes) in a population of 1,000 erythrocytes was scored for each dose group as a measure of bone marrow toxicity.

The results were tabulated as the mean of the pooled results from all animals within a treatment group plus or minus the standard error of the mean. The frequency of micronucleated cells among NCEs was analyzed by a statistical software package that tested for increasing trend over exposure groups with a one-tailed Cochran-Armitage trend test, followed by pairwise comparisons between each exposed group and the control group. In the presence of excess binomial variation, as detected by a binomial dispersion test, the binomial variance of the Cochran-Armitage test was adjusted upward in proportion to the excess variation. In the micronucleus test, an individual trial is considered positive if the trend test $\mathrm{P}$ value is less than or equal to 0.025 or if the $\mathrm{P}$ value for any single exposed group is less than or equal to 0.025 divided by the number of exposed groups. Ultimately, the final call is determined by the scientific staff after considering the results of statistical analyses, the reproducibility of any effects observed, and the magnitudes of those effects.

\section{Evaluation Protocol}

These are the basic guidelines for arriving at an overall assay result for assays performed by the National Toxicology Program. Statistical as well as biological factors are considered. For an individual assay, the statistical procedures for data analysis have been described in the preceding protocols. There have been instances, however, in which multiple samples of a chemical were tested in the same assay, and different results were obtained among these samples and/or among laboratories. Results from more than one aliquot or from more than one laboratory are not simply combined into an overall result. Rather, all the data are critically evaluated, particularly with regard to pertinent protocol variations, in determining the weight of evidence for an overall conclusion of chemical activity in an assay. In addition to multiple aliquots, the in vitro assays have another variable that must be considered in arriving at an overall test result. In vitro assays are conducted with and without exogenous metabolic activation. Results obtained in the absence of activation are not combined with results obtained in the presence of activation; each testing condition is evaluated separately. The summary table in the Abstract of this Toxicity Study Report presents a result that represents a scientific judgment of the overall evidence for activity of the chemical in an assay. 


\section{Results}

\section{Rats}

\section{Two-week Dermal Study}

All rats survived to the end of the study (Table 2). The final mean body weights and body weight gains of all dosed groups were similar to those of the vehicle control groups. There were no clinical findings related to $o$-chloropyridine administration.

The absolute and relative liver weights of 50 (absolute, 10.6\%) and $100 \mathrm{mg} / \mathrm{kg}$ (absolute, 9.6\%) males were significantly greater than those of the vehicle controls (Table 3 and Table $\mathrm{C}-1$ ). Absolute and relative organ weights of all dosed groups of females were similar to those of the vehicle controls (Table C-1). No gross or microscopic lesions were considered related to $o$-chloropyridine administration.

Table 2. Survival and Body Weights of Rats in the Two-week Dermal Study of $o$-Chloropyridine ${ }^{\text {a }}$

\begin{tabular}{cccccc}
\hline $\begin{array}{c}\text { Dose } \\
(\mathbf{m g} / \mathbf{k g})\end{array}$ & Survival $^{\mathbf{b}}$ & $\begin{array}{c}\text { Initial Body } \\
\text { Weight (g) }\end{array}$ & $\begin{array}{c}\text { Final Body } \\
\text { Weight (g) }\end{array}$ & $\begin{array}{c}\text { Change in Body } \\
\text { Weight (g) }\end{array}$ & $\begin{array}{c}\text { Final Weight } \\
\text { Relative to } \\
\text { Controls (\%) }\end{array}$ \\
\hline Male & & & & & \\
0 & $5 / 5$ & $90 \pm 3$ & $157 \pm 6$ & $68 \pm 3$ & 101 \\
6.25 & $5 / 5$ & $88 \pm 4$ & $159 \pm 6$ & $71 \pm 3$ & 100 \\
12.5 & $5 / 5$ & $87 \pm 3$ & $157 \pm 4$ & $70 \pm 2$ & 97 \\
25 & $5 / 5$ & $85 \pm 3$ & $153 \pm 3$ & $68 \pm 2$ & 101 \\
50 & $5 / 5$ & $88 \pm 2$ & $158 \pm 5$ & $71 \pm 3$ & 101 \\
100 & $5 / 5$ & $87 \pm 3$ & $158 \pm 4$ & $71 \pm 2$ & 101 \\
\hline Female & & & & & 100 \\
0 & $5 / 5$ & $82 \pm 3$ & $120 \pm 2$ & $39 \pm 2$ & 102 \\
6.25 & $5 / 5$ & $80 \pm 2$ & $121 \pm 1$ & $41 \pm 2$ & 100 \\
12.5 & $5 / 5$ & $81 \pm 3$ & $120 \pm 3$ & $40 \pm 1$ & 100 \\
25 & $5 / 5$ & $81 \pm 4$ & $122 \pm 4$ & $41 \pm 2$ & \\
50 & $5 / 5$ & $80 \pm 2$ & $120 \pm 2$ & $40 \pm 2$ & $40 \pm 1$ \\
100 & $5 / 5$ & $80 \pm 2$ & $120 \pm 2$ & & \\
\hline
\end{tabular}

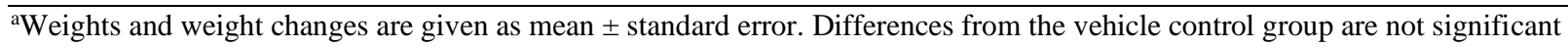
by Dunnett's test.

${ }^{b}$ Number of animals surviving at 17 days/number initially in group. 
Table 3. Liver Weights and Liver-Weight-to-Body-Weight Ratios for Male Rats in the Two-week Dermal Study of $o$-Chloropyridine ${ }^{a}$

\begin{tabular}{|c|c|c|c|c|c|c|}
\hline & $\begin{array}{l}\text { Vehicle } \\
\text { Control }\end{array}$ & $6.25 \mathrm{mg} / \mathrm{kg}$ & $12.5 \mathrm{mg} / \mathrm{kg}$ & $25 \mathrm{mg} / \mathrm{kg}$ & $50 \mathrm{mg} / \mathrm{kg}$ & $100 \mathrm{mg} / \mathrm{kg}$ \\
\hline n & 5 & 5 & 5 & 5 & 5 & 5 \\
\hline $\begin{array}{l}\text { Necropsy } \\
\text { body wt }\end{array}$ & $157 \pm 6$ & $159 \pm 6$ & $157 \pm 4$ & $153 \pm 3$ & $158 \pm 5$ & $158 \pm 4$ \\
\hline \multicolumn{7}{|l|}{ Liver } \\
\hline Absolute & $8.02 \pm 0.28$ & $8.13 \pm 0.18$ & $8.25 \pm 0.23$ & $8.03 \pm 0.16$ & $8.87 \pm 0.35 *$ & $8.79 \pm 0.11^{*}$ \\
\hline Relative & $51.083 \pm 0.188$ & $51.251 \pm 0.857$ & $52.473 \pm 1.018$ & $52.428 \pm 0.422$ & $55.961 \pm 0.567 * *$ & $55.732 \pm 0.777 * *$ \\
\hline
\end{tabular}

Exposure concentration selection rationale: Exposure concentrations in the 3-month drinking water study were selected for comparison to the 3-month pyridine study results ${ }^{33}$.

\section{Three-month Drinking Water Study}

All rats survived to the end of the study (Table 4). Final mean body weights and body weight gains of male and female rats exposed to $1,000 \mathrm{ppm}$ were significantly less than those of the controls (Table 4 and Figure 2). Water consumption was lower in the 1,000 ppm groups, compared to controls, particularly during week 1 of the study. In male and female rats, water consumption in the 1,000 ppm groups was approximately half that of controls in the first week, increasing in week 2 . By week 14, water consumption by female rats had completely recovered while water consumption by male rats was only slightly less than that of controls (Table 4, Table G-1, and Table G-2). Drinking water concentrations of 10, 30, 100, 300, and 1,000 ppm resulted in average daily doses of approximately $1,3,9,25$, and $65 \mathrm{mg} o$-chloropyridine/kg body weight to males and 1,3,9,27, and $70 \mathrm{mg} / \mathrm{kg}$ to females. In the $1,000 \mathrm{ppm}$ groups, thinness was noted in seven of 10 males and all females on day 8 and also in five of 10 males on day 64; thinness noted on day 8 was likely due to dehydration from reduction in water consumption. Thinness was diagnosed based on the degree of visibility of the vertebrae and pelvic bones. 
Table 4. Survival, Body Weights, and Water Consumption of Rats in the Three-month Drinking Water Study of $o$-Chloropyridine ${ }^{\text {a }}$

\begin{tabular}{cccccccc}
\hline $\begin{array}{c}\text { Concentration } \\
\text { (ppm) }\end{array}$ & Survival $^{\mathbf{b}}$ & $\begin{array}{c}\text { Initial } \\
\text { Body } \\
\text { Weight (g) }\end{array}$ & $\begin{array}{c}\text { Final Body } \\
\text { Weight (g) }\end{array}$ & $\begin{array}{c}\text { Change in } \\
\text { Body } \\
\text { Weight (g) }\end{array}$ & $\begin{array}{c}\text { Final Weight } \\
\text { Relative to } \\
\text { Controls (\%) }\end{array}$ & $\begin{array}{c}\text { Water } \\
\text { Consumption } \\
\text { Week 1 }\end{array}$ & $\begin{array}{c}\text { Water } \\
\text { Consumption } \\
\text { Week 14 }\end{array}$ \\
\hline Male & & & & & & & \\
0 & $10 / 10$ & $90 \pm 2$ & $320 \pm 6$ & $230 \pm 5$ & & 14.7 & 17.3 \\
10 & $10 / 10$ & $93 \pm 2$ & $326 \pm 7$ & $233 \pm 6$ & 100 & 15.5 & 17.7 \\
30 & $10 / 10$ & $92 \pm 2$ & $326 \pm 8$ & $234 \pm 7$ & 102 & 15.1 & 13.6 \\
100 & $10 / 10$ & $91 \pm 2$ & $326 \pm 8$ & $235 \pm 9$ & 102 & 15.4 & 18.1 \\
300 & $10 / 10$ & $91 \pm 2$ & $309 \pm 7$ & $218 \pm 7$ & 97 & 13.6 & 17.5 \\
1,000 & $10 / 10$ & $94 \pm 3$ & $268 \pm 6^{* *}$ & $173 \pm 4^{* *}$ & 84 & 6.5 & 15.1 \\
\hline Female & & & & & & & \\
0 & $10 / 10$ & $86 \pm 2$ & $195 \pm 4$ & $109 \pm 3$ & & 12.5 & 13.4 \\
10 & $10 / 10$ & $88 \pm 2$ & $200 \pm 2$ & $112 \pm 3$ & 103 & 13.8 & 17.7 \\
30 & $10 / 10$ & $87 \pm 1$ & $200 \pm 4$ & $113 \pm 4$ & 103 & 13.0 & 14.8 \\
100 & $10 / 10$ & $88 \pm 2$ & $196 \pm 2$ & $108 \pm 3$ & 101 & 12.6 & 17.4 \\
300 & $10 / 10$ & $86 \pm 2$ & $190 \pm 3$ & $104 \pm 3$ & 97 & 12.2 & 12.7 \\
1,000 & $10 / 10$ & $87 \pm 2$ & $174 \pm 2^{* *}$ & $87 \pm 3^{* *}$ & 89 & 6.1 & 15.8 \\
\hline
\end{tabular}

**Significantly different $(\mathrm{P} \leq 0.01)$ from the control group by Williams' test.

aWeights and weight changes are given as mean \pm standard error. Water consumption is expressed as grams per animal per day.

bumber of animals surviving at 14 weeks/number initially in group. 

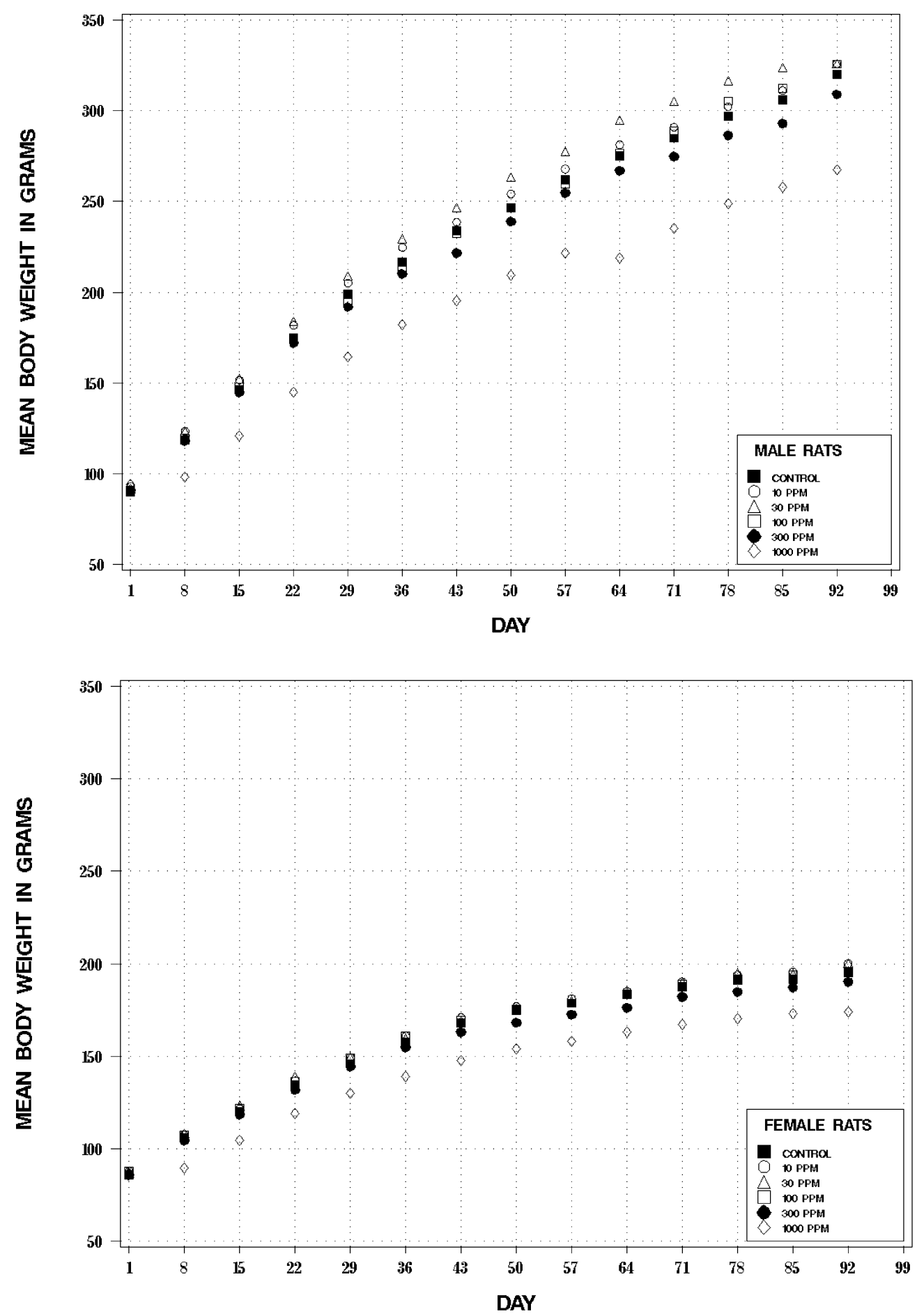

Figure 2. Growth Curves for Rats Exposed to o-Chloropyridine in Drinking Water for Three Months

On day 4, the hematology findings demonstrated a small (approximately 10\%) increase in the circulating erythron (i.e., an erythrocytosis). The erythrocytosis was evidenced by increases in 
hematocrit values, hemoglobin concentrations, and erythrocyte counts of the 1,000 ppm males (Table 5 and Table B-1). Additionally, urea nitrogen concentrations increased in the 1,000 ppm males on day 4 (Table B-1). These early changes may be the result of dehydration; there was an approximate $50 \%$ reduction in water intake during week 1 of the study (Table 4). The increases in the erythron and serum urea nitrogen were transient and disappeared by day 22, being replaced by an apparent dose-related erythron decrease in male and female rats exposed to $100 \mathrm{ppm}$ or greater. The erythron decrease was small $(\leq 10 \%)$ and was demonstrated by decreased hematocrit values and hemoglobin concentrations. The erythrocyte counts were unaffected in this case; however, there were small reductions $(\leq 5 \%)$ in erythrocyte size (microcytosis), evidenced by decreases in the mean cell volume. By week 14, the erythron decrease had progressed to include decreases in the erythrocyte count and increases in reticulocyte count in the $300 \mathrm{ppm}$ male and $1,000 \mathrm{ppm}$ male and female rats. While the magnitude of these changes were small, they were supported by histologic changes in the bone marrow and spleen.

An exposure concentration-related hyperalbuminemia, demonstrated by increased serum albumin concentrations, occurred in the $30 \mathrm{ppm}$ or greater males and females at week 14; the 1,000 ppm groups demonstrated a $10 \%$ to $15 \%$ increase (Table 5 and Table B-1). The hyperalbuminemia was progressive and, on day 4, involved a minimal 5\% increase in the 1,000 ppm males only. By day 22, the 100,300, and 1,000 ppm males and females were affected. At week 14, the increased serum albumin was accompanied by proportional increases in the serum total protein concentration. There was also evidence of a potential alteration in hepatic function demonstrated by increased serum bile acid concentrations. The most consistent change occurred on day 4 , involving the 300 and 1,000 ppm males and females. Though the mechanism was unknown, the effect leading to the bile acid increase was transient in males, and bile acid concentrations demonstrated amelioration by day 22 and were essentially resolved at week 14 . Bile acid concentrations remained higher in female mice at week 14 compared to controls. 
Table 5. Selected Clinical Pathology Data for Rats in the Three-month Drinking Water Study of $o$-Chloropyridine ${ }^{\text {a }}$

\begin{tabular}{|c|c|c|c|c|c|c|}
\hline & 0 ppm & $10 \mathrm{ppm}$ & 30 ppm & $100 \mathrm{ppm}$ & 300 ppm & 1,000 ppm \\
\hline \multicolumn{7}{|l|}{ Male } \\
\hline \multicolumn{7}{|c|}{ Hematology } \\
\hline \multicolumn{7}{|l|}{$\mathbf{n}$} \\
\hline Day 4 & 10 & 10 & 10 & 9 & 9 & 10 \\
\hline Day 22 & 10 & 10 & 10 & 10 & 10 & 10 \\
\hline Week 14 & 9 & 10 & 10 & 10 & 10 & 10 \\
\hline \multicolumn{7}{|c|}{ Hematocrit (\%) } \\
\hline Day 4 & $40.0 \pm 0.4$ & $39.8 \pm 0.4$ & $40.5 \pm 0.6$ & $38.8 \pm 0.5$ & $39.6 \pm 0.5$ & $43.3 \pm 0.9^{*}$ \\
\hline Day 22 & $43.4 \pm 0.4$ & $42.7 \pm 0.4$ & $42.6 \pm 0.4$ & $41.2 \pm 0.6^{* *}$ & $41.5 \pm 0.5^{* *}$ & $39.6 \pm 0.4 * *$ \\
\hline Week 14 & $45.5 \pm 0.6$ & $45.2 \pm 0.3$ & $46.3 \pm 0.3$ & $44.5 \pm 0.9$ & $42.9 \pm 0.4^{* *}$ & $41.7 \pm 0.4^{* *}$ \\
\hline \multicolumn{7}{|c|}{ Hemoglobin (g/dL) } \\
\hline Day 4 & $13.4 \pm 0.1$ & $13.4 \pm 0.1$ & $13.6 \pm 0.2$ & $13.1 \pm 0.2$ & $13.3 \pm 0.2$ & $14.5 \pm 0.3^{*}$ \\
\hline Day 22 & $14.8 \pm 0.1$ & $14.5 \pm 0.1$ & $14.5 \pm 0.1$ & $14.1 \pm 0.2 * *$ & $14.1 \pm 0.2^{* *}$ & $13.5 \pm 0.1^{* *}$ \\
\hline Week 14 & $15.2 \pm 0.2$ & $15.1 \pm 0.1$ & $15.4 \pm 0.1$ & $15.0 \pm 0.3$ & $14.4 \pm 0.1 * *$ & $13.9 \pm 0.1 * *$ \\
\hline \multicolumn{7}{|c|}{ Erythrocytes $\left(10^{6} / \mu \mathrm{L}\right)$} \\
\hline Day 4 & $6.80 \pm 0.08$ & $6.85 \pm 0.10$ & $6.92 \pm 0.10$ & $6.72 \pm 0.09$ & $6.95 \pm 0.10$ & $7.53 \pm 0.14 * *$ \\
\hline Day 22 & $7.50 \pm 0.07$ & $7.35 \pm 0.09$ & $7.37 \pm 0.08$ & $7.18 \pm 0.11^{*}$ & $7.30 \pm 0.08$ & $7.25 \pm 0.08$ \\
\hline Week 14 & $8.91 \pm 0.11$ & $8.75 \pm 0.07$ & $9.04 \pm 0.08$ & $8.76 \pm 0.15$ & $8.51 \pm 0.06^{* *}$ & $8.42 \pm 0.09 * *$ \\
\hline \multicolumn{7}{|c|}{ Reticulocytes $\left(10^{6} / \mu \mathrm{L}\right)$} \\
\hline Day 4 & $0.46 \pm 0.04$ & $0.45 \pm 0.04$ & $0.54 \pm 0.02$ & $0.50 \pm 0.05$ & $0.46 \pm 0.03$ & $0.39 \pm 0.02$ \\
\hline Day 22 & $0.33 \pm 0.04$ & $0.33 \pm 0.02$ & $0.28 \pm 0.02$ & $0.30 \pm 0.02$ & $0.36 \pm 0.04$ & $0.41 \pm 0.02$ \\
\hline Week 14 & $0.26 \pm 0.01$ & $0.27 \pm 0.02$ & $0.27 \pm 0.02$ & $0.25 \pm 0.02$ & $0.37 \pm 0.02 * *$ & $0.32 \pm 0.03^{*}$ \\
\hline \multicolumn{7}{|c|}{ Mean cell volume (fL) } \\
\hline Day 4 & $58.8 \pm 0.3$ & $58.1 \pm 0.2$ & $58.5 \pm 0.3$ & $57.8 \pm 0.3^{*}$ & $56.9 \pm 0.2 * *$ & $57.5 \pm 0.2 * *$ \\
\hline Day 22 & $57.9 \pm 0.3$ & $58.0 \pm 0.3$ & $57.7 \pm 0.2$ & $57.3 \pm 0.3$ & $56.8 \pm 0.3 * *$ & $54.7 \pm 0.2 * *$ \\
\hline Week 14 & $51.2 \pm 0.2$ & $51.6 \pm 0.2$ & $51.3 \pm 0.2$ & $50.8 \pm 0.3$ & $50.5 \pm 0.2^{*}$ & $49.3 \pm 0.3 * *$ \\
\hline \multicolumn{7}{|c|}{ Mean cell hemoglobin $(\mathrm{pg})$} \\
\hline Day 4 & $19.7 \pm 0.1$ & $19.5 \pm 0.1$ & $19.6 \pm 0.1$ & $19.5 \pm 0.1$ & $19.1 \pm 0.1 * *$ & $19.3 \pm 0.1 * *$ \\
\hline Day 22 & $19.8 \pm 0.1$ & $19.8 \pm 0.1$ & $19.7 \pm 0.1$ & $19.6 \pm 0.1$ & $19.4 \pm 0.0^{* *}$ & $18.7 \pm 0.1 * *$ \\
\hline Week 14 & $17.0 \pm 0.0$ & $17.2 \pm 0.1$ & $17.1 \pm 0.1$ & $17.1 \pm 0.1$ & $16.9 \pm 0.1$ & $16.5 \pm 0.1 * *$ \\
\hline \multicolumn{7}{|c|}{ Mean cell hemoglobin concentration $(\mathrm{g} / \mathrm{dL})$} \\
\hline Day 4 & $33.6 \pm 0.1$ & $33.6 \pm 0.1$ & $33.5 \pm 0.1$ & $33.7 \pm 0.1$ & $33.6 \pm 0.1$ & $33.5 \pm 0.1$ \\
\hline Day 22 & $34.1 \pm 0.1$ & $34.1 \pm 0.1$ & $34.0 \pm 0.1$ & $34.2 \pm 0.1$ & $34.1 \pm 0.1$ & $34.1 \pm 0.1$ \\
\hline Week 14 & $33.3 \pm 0.1$ & $33.3 \pm 0.1$ & $33.3 \pm 0.1$ & $33.6 \pm 0.1$ & $33.5 \pm 0.1$ & $33.4 \pm 0.1$ \\
\hline
\end{tabular}


o-Chloropyridine, NTP TOX 83

\begin{tabular}{|c|c|c|c|c|c|c|}
\hline & 0 ppm & 10 ppm & 30 ppm & 100 ppm & 300 ppm & 1,000 ppm \\
\hline \multicolumn{7}{|c|}{ Clinical Chemistry } \\
\hline n & 10 & 10 & 10 & 10 & 10 & 10 \\
\hline \multicolumn{7}{|c|}{ Total protein $(\mathrm{g} / \mathrm{dL})$} \\
\hline Day 4 & $5.7 \pm 0.1$ & $5.7 \pm 0.0$ & $5.8 \pm 0.1$ & $5.8 \pm 0.1$ & $5.9 \pm 0.1$ & $5.9 \pm 0.1$ \\
\hline Day 22 & $6.4 \pm 0.0$ & $6.4 \pm 0.1$ & $6.5 \pm 0.1$ & $6.5 \pm 0.1$ & $6.8 \pm 0.1 *$ & $6.6 \pm 0.1$ \\
\hline Week 14 & $7.3 \pm 0.1$ & $7.3 \pm 0.1$ & $7.5 \pm 0.1$ & $7.8 \pm 0.1 * *$ & $8.0 \pm 0.1^{* *}$ & $8.3 \pm 0.1 * *$ \\
\hline \multicolumn{7}{|c|}{ Albumin (g/dL) } \\
\hline Day 4 & $4.1 \pm 0.0$ & $4.1 \pm 0.0$ & $4.1 \pm 0.0$ & $4.1 \pm 0.0$ & $4.2 \pm 0.0$ & $4.3 \pm 0.1^{*}$ \\
\hline Day 22 & $4.4 \pm 0.0$ & $4.4 \pm 0.1$ & $4.5 \pm 0.1$ & $4.6 \pm 0.0 * *$ & $4.7 \pm 0.1^{* *}$ & $4.7 \pm 0.1 * *$ \\
\hline Week 14 & $4.9 \pm 0.0$ & $4.9 \pm 0.0$ & $5.0 \pm 0.0^{*}$ & $5.2 \pm 0.1 * *$ & $5.4 \pm 0.1^{* *}$ & $5.7 \pm 0.0^{* *}$ \\
\hline \multicolumn{7}{|c|}{ Bile acids $(\mu \mathrm{mol} / \mathrm{L})$} \\
\hline Day 4 & $3.8 \pm 1.4$ & $26.5 \pm 2.1$ & $26.4 \pm 1.3$ & $33.8 \pm 2.2 * *$ & $49.8 \pm 6.4^{* *}$ & $41.0 \pm 5.4^{* *}$ \\
\hline Day 22 & $25.7 \pm 1.1$ & $25.5 \pm 1.2$ & $31.0 \pm 2.6$ & $28.0 \pm 2.0$ & $34.9 \pm 2.9 * *$ & $39.1 \pm 2.8^{* *}$ \\
\hline Week 14 & $31.1 \pm 3.4$ & $26.0 \pm 1.0$ & $29.0 \pm 1.9$ & $26.1 \pm 0.8$ & $33.1 \pm 3.1$ & $39.2 \pm 2.9$ \\
\hline
\end{tabular}

\section{Female}

$\mathbf{n}$

\begin{tabular}{lcccccc} 
Day 4 & 10 & 9 & 10 & 10 & 10 & 9 \\
Day 22 & 10 & 10 & 10 & 10 & 10 & 10 \\
Week 14 & 10 & 10 & 10 & 10 & 10 & 10 \\
\hline
\end{tabular}

Hematocrit (\%)

Day 4

Day 22

Week 14

Hemoglobin $(\mathrm{g} / \mathrm{dL})$

Day 4

Day 22

Week 14

Erythrocytes $\left(10^{6} / \mu \mathrm{L}\right)$

Day 4

Day 22

Week 14

Reticulocytes $\left(10^{6} / \mu \mathrm{L}\right)$

Day 4

Day 22

Week 14
$42.6 \pm 0.5 \quad 41.4 \pm 0.5 \quad 42.6 \pm 0.4 \quad 40.6 \pm 1.1$

$44.9 \pm 0.3$

$44.3 \pm 0.8$

$45.9 \pm 0.4$

$44.4 \pm 0.4$

$43.5 \pm 0.3^{*}$

$43.7 \pm 0.7$

$45.1 \pm 0.3$

$14.4 \pm 0.2 \quad 14.1 \pm 0.2 \quad 14.4 \pm 0.2$

$13.8 \pm 0.4$

$15.2 \pm 0.1$

$15.0 \pm 0.2$

$15.1 \pm 0.2 \quad 15.4 \pm 0.1 \quad 15.4 \pm 0.1$

$7.33 \pm 0.09 \quad 7.16 \pm 0.09 \quad 7.35 \pm 0.07$

$7.80 \pm 0.07 \quad 7.95 \pm 0.08 \quad 7.75 \pm 0.08$

$7.02 \pm 0.19$

$7.70 \pm 0.06$

$8.27 \pm 0.12$

$8.30 \pm 0.13 \quad 8.50 \pm 0.05 \quad 8.48 \pm 0.07$

$0.36 \pm 0.02$

$0.19 \pm 0.01$

$0.23 \pm 0.02$

$0.36 \pm 0.01 \quad 0.35 \pm 0.03 \quad 0.35 \pm 0.01$

$\begin{array}{lll}0.20 \pm 0.01 & 0.20 \pm 0.01 & 0.20 \pm 0.01\end{array}$

$0.23 \pm 0.02 \quad 0.26 \pm 0.02 \quad 0.21 \pm 0.02$

\section{$39.8 \pm 0.8^{*}$}

$42.4 \pm 0.6^{* *}$

$42.6 \pm 0.2 * *$

$13.6 \pm 0.3$

$14.8 \pm 0.2 *$

$14.7 \pm 0.1^{*}$

$6.93 \pm 0.15$

$7.51 \pm 0.13$

$8.17 \pm 0.03$

$44.9 \pm 0.8$

$42.4 \pm 1.1^{* *}$

$40.7 \pm 0.4 * *$

$0.32 \pm 0.02$

$0.28 \pm 0.01 * *$

$0.22 \pm 0.03$

$0.29 \pm 0.02 *$

$0.26 \pm 0.03$

$0.33 \pm 0.02 * *$ 
$o$-Chloropyridine, NTP TOX 83

\begin{tabular}{lcccccc}
\hline \multicolumn{1}{c}{$\mathbf{0 ~} \mathbf{~ p p m}$} & $\mathbf{1 0} \mathbf{~ p p m}$ & $\mathbf{3 0} \mathbf{~ p p m}$ & $\mathbf{1 0 0} \mathbf{~ p p m}$ & $\mathbf{3 0 0} \mathbf{~ p p m}$ & $\mathbf{1 , 0 0 0} \mathbf{~ p p m}$ \\
\hline Mean cell volume (fL) & & & & & & \\
Day 4 & $58.0 \pm 0.2$ & $57.8 \pm 0.2$ & $57.8 \pm 0.3$ & $57.8 \pm 0.3$ & $57.6 \pm 0.2$ & $57.1 \pm 0.2^{* *}$ \\
Day 22 & $57.5 \pm 0.2$ & $57.7 \pm 0.2$ & $57.4 \pm 0.3$ & $56.6 \pm 0.2^{*}$ & $56.6 \pm 0.4^{*}$ & $54.6 \pm 0.2^{* *}$ \\
Week 14 & $53.5 \pm 0.2$ & $53.2 \pm 0.1$ & $53.2 \pm 0.1$ & $52.8 \pm 0.1^{* *}$ & $52.1 \pm 0.2^{* *}$ & $51.5 \pm 0.2^{* *}$ \\
Mean cell hemoglobin (pg) & & & & & \\
Day 4 & $19.7 \pm 0.1$ & $19.7 \pm 0.1$ & $19.6 \pm 0.1$ & $19.7 \pm 0.1$ & $19.7 \pm 0.1$ & $19.4 \pm 0.1$ \\
Day 22 & $19.9 \pm 0.1$ & $20.0 \pm 0.1$ & $19.9 \pm 0.1$ & $19.8 \pm 0.1$ & $19.7 \pm 0.1$ & $19.0 \pm 0.1^{* *}$ \\
Week 14 & $18.2 \pm 0.1$ & $18.1 \pm 0.1$ & $18.1 \pm 0.1$ & $18.1 \pm 0.1$ & $18.0 \pm 0.1$ & $17.6 \pm 0.1^{* *}$ \\
Mean cell hemoglobin concentration (g/dL) & & & & \\
Day 4 & $33.8 \pm 0.1$ & $34.0 \pm 0.1$ & $33.8 \pm 0.1$ & $34.1 \pm 0.1$ & $34.3 \pm 0.1 *$ & $34.0 \pm 0.1$ \\
Day 22 & $34.5 \pm 0.1$ & $34.6 \pm 0.1$ & $34.7 \pm 0.1$ & $35.0 \pm 0.1 *$ & $34.8 \pm 0.1$ & $34.7 \pm 0.1$ \\
Week 14 & $34.1 \pm 0.2$ & $34.1 \pm 0.1$ & $34.1 \pm 0.1$ & $34.4 \pm 0.1$ & $34.5 \pm 0.2$ & $34.0 \pm 0.1$ \\
\hline
\end{tabular}

\section{Clinical Chemistry}

\begin{tabular}{lcccccc}
$\mathbf{n}$ & 10 & 10 & 10 & 10 & 10 & 10 \\
\hline Total protein $(\mathrm{g} / \mathrm{dL})$ & & & & & & \\
$\quad$ Day 4 & $5.9 \pm 0.1$ & $5.8 \pm 0.0$ & $6.0 \pm 0.1$ & $5.9 \pm 0.0$ & $5.9 \pm 0.1$ & $5.8 \pm 0.1$ \\
Day 22 & $6.3 \pm 0.1$ & $6.6 \pm 0.1$ & $6.5 \pm 0.2$ & $6.5 \pm 0.1$ & $6.5 \pm 0.1$ & $6.6 \pm 0.1$ \\
$\quad$ Week 14 & $6.9 \pm 0.1$ & $7.2 \pm 0.1$ & $7.5 \pm 0.1^{* *}$ & $7.4 \pm 0.1^{* *}$ & $7.5 \pm 0.1^{* *}$ & $7.8 \pm 0.1^{* *}$ \\
Albumin (g/dL) & & & & & & \\
Day 4 & $4.3 \pm 0.0$ & $4.3 \pm 0.0$ & $4.5 \pm 0.0^{*}$ & $4.3 \pm 0.0$ & $4.3 \pm 0.0$ & $4.4 \pm 0.1$ \\
Day 22 & $4.6 \pm 0.1$ & $4.7 \pm 0.0$ & $4.7 \pm 0.1$ & $4.8 \pm 0.1^{* *}$ & $4.8 \pm 0.1^{*}$ & $4.9 \pm 0.1^{* *}$ \\
Week 14 & $5.1 \pm 0.2$ & $5.1 \pm 0.1$ & $5.3 \pm 0.1^{*}$ & $5.4 \pm 0.1^{* *}$ & $5.4 \pm 0.1^{* *}$ & $5.7 \pm 0.1^{* *}$ \\
Bile acids $(\mu \mathrm{mol} / \mathrm{L})$ & & & & & & \\
Day 4 & $26.4 \pm 1.3$ & $24.6 \pm 0.8$ & $27.3 \pm 2.2$ & $26.9 \pm 1.6$ & $40.3 \pm 3.8^{* *}$ & $31.7 \pm 2.8^{*}$ \\
Day 22 & $24.0 \pm 0.8$ & $30.1 \pm 1.5^{*}$ & $27.0 \pm 1.4$ & $23.1 \pm 1.1$ & $29.1 \pm 1.7$ & $27.2 \pm 1.0$ \\
Week 14 & $27.0 \pm 1.7$ & $34.3 \pm 2.9$ & $32.6 \pm 1.4$ & $34.8 \pm 3.3$ & $30.8 \pm 1.5$ & $36.4 \pm 3.2^{*}$ \\
\hline
\end{tabular}

*Significantly different $(\mathrm{P} \leq 0.05)$ from the control group by Dunn's or Shirley's test.

$* * \mathrm{P} \leq 0.01$.

${ }^{a}$ Data are presented as mean \pm standard error. Statistical tests were performed on unrounded data. 
The absolute and relative (except $100 \mathrm{ppm}$ ) right kidney weights of all exposed groups of males and of female groups exposed to $30 \mathrm{ppm}$ or greater were significantly greater than those of the control groups (Table 6 and Table C-2). Absolute and relative liver weights of males exposed to $100 \mathrm{ppm}$ or greater and females exposed to $30 \mathrm{ppm}$ or greater were significantly greater than those of the controls.

Epididymal sperm counts (total and sperm/mg cauda) were significantly lower in males exposed to 1,000 ppm (Table 7 and Table D-1). There were no test article-related changes in the estrous cyclicity of rats exposed to $o$-chloropyridine (Table D-2, Table D-3, and Figure D-1).

Table 6. Selected Organ Weights and Organ-Weight-to-Body-Weight Ratios for Rats in the Threemonth Drinking Water Study of $o$-Chloropyridine ${ }^{a}$

\begin{tabular}{|c|c|c|c|c|c|c|}
\hline & O ppm & $10 \mathrm{ppm}$ & $30 \mathrm{ppm}$ & 100 ppm & 300 ppm & 1,000 ppm \\
\hline n & 1 & 10 & 10 & 10 & 10 & 10 \\
\hline \multicolumn{7}{|l|}{ Male } \\
\hline Necropsy body wt & $320 \pm 6$ & $326 \pm 7$ & $326 \pm 8$ & $326 \pm 8$ & $309 \pm 7$ & $268 \pm 6^{* *}$ \\
\hline \multicolumn{7}{|l|}{ R. Kidney } \\
\hline Absolute & $0.95 \pm 0.01$ & $1.02 \pm 0.02 *$ & $1.04 \pm 0.03 *$ & $1.01 \pm 0.02 *$ & $1.02 \pm 0.03 *$ & $1.07 \pm 0.02 * *$ \\
\hline Relative & $2.978 \pm 0.065$ & $3.144 \pm 0.036^{*}$ & $3.172 \pm 0.052 *$ & $3.110 \pm 0.081$ & $3.304 \pm 0.030^{* *}$ & $4.012 \pm 0.052 * *$ \\
\hline \multicolumn{7}{|l|}{ Liver } \\
\hline Absolute & $10.91 \pm 0.29$ & $11.76 \pm 0.29$ & $10.87 \pm 0.78$ & $13.17 \pm 0.35^{* *}$ & $13.51 \pm 0.40^{* *}$ & $14.33 \pm 0.36^{* *}$ \\
\hline Relative & $34.101 \pm 0.737$ & $36.114 \pm 0.615$ & $33.092 \pm 1.788$ & $40.530 \pm 1.053 * *$ & $43.672 \pm 0.620 * *$ & $53.553 \pm 0.653 * *$ \\
\hline
\end{tabular}

Female

Necropsy body wt

$195+4$

$200 \pm 2$

$200 \pm 4$

$196 \pm 2$

$190 \pm 3$

$174 \pm 2 * *$

R. Kidney

$\begin{array}{lcccccc}\text { Absolute } & 0.65 \pm 0.02 & 0.67 \pm 0.01 & 0.70 \pm 0.01 * & 0.69 \pm 0.02 * & 0.71 \pm 0.01 * * & 0.74 \pm 0.01 * * \\ \text { Relative } & 3.317 \pm 0.047 & 3.347 \pm 0.032 & 3.472 \pm 0.050 * & 3.530 \pm 0.051 * * & 3.714 \pm 0.058 * * & 4.259 \pm 0.040 * *\end{array}$

Liver

\begin{tabular}{lcccccc} 
Absolute & $5.80 \pm 0.22$ & $6.14 \pm 0.14$ & $6.77 \pm 0.17 * *$ & $6.89 \pm 0.12 * *$ & $7.94 \pm 0.34 * *$ & $9.06 \pm 0.21 * *$ \\
Relative & $29.632 \pm 0.735$ & $30.698 \pm 0.619$ & $33.784 \pm 0.410 * *$ & $35.152 \pm 0.373 * *$ & $41.684 \pm 1.375 * *$ & $52.086 \pm 0.597 * *$ \\
\hline
\end{tabular}

*Significantly different $(\mathrm{P} \leq 0.05)$ from the control group by Williams' test.

$* * \mathrm{P} \leq 0.01$.

${ }^{\mathrm{a}}$ Organ weights (absolute weights) and body weights are given in grams; organ-weight-to-body-weight ratios (relative weights) are given as mg organ weight/g body weight (mean \pm standard error). 
Table 7. Summary of Reproductive Tissue Evaluations for Male Rats in the Three-month Drinking Water Study of $o$-Chloropyridine ${ }^{a}$

\begin{tabular}{lcccc}
\hline & $\mathbf{0 ~} \mathbf{~ p m}$ & $\mathbf{1 0 0} \mathbf{~ p p m}$ & $\mathbf{3 0 0} \mathbf{~ p p m}$ & $\mathbf{1 , 0 0 0} \mathbf{~ p p m}$ \\
\hline $\mathbf{n}$ & 10 & 10 & 10 & 10 \\
\hline Weights (g) & & & & \\
$\quad$ Necropsy body wt & $320 \pm 6$ & $326 \pm 8$ & $309 \pm 7$ & $268 \pm 6^{* *}$ \\
L. Cauda epididymis & $0.1510 \pm 0.0067$ & $0.1563 \pm 0.0040$ & $0.1548 \pm 0.0041$ & $0.1503 \pm 0.0037$ \\
L. Epididymis & $0.4239 \pm 0.0177$ & $0.4579 \pm 0.0097$ & $0.4457 \pm 0.0063$ & $0.4222 \pm 0.0079$ \\
L. Testis & $1.4547 \pm 0.0387$ & $1.5359 \pm 0.0227$ & $1.4795 \pm 0.0269$ & $1.4671 \pm 0.0212$ \\
Spermatid measurements & & & & \\
$\quad$ Spermatid heads (106/testis) & $169.75 \pm 7.20$ & $182.13 \pm 5.88$ & $170.88 \pm 4.14$ & $166.75 \pm 4.25$ \\
$\quad$ Spermatid heads (10\%/g testis) & $129.7 \pm 3.6$ & $131.9 \pm 4.6$ & $126.8 \pm 2.8$ & $125.6 \pm 3.0$ \\
Epididymal spermatozoal measurements & & & & \\
$\quad$ Sperm motility (\%) & $77.6 \pm 1.0$ & $71.7 \pm 8.0$ & $76.9 \pm 1.4$ & $77.4 \pm 1.0$ \\
Sperm (10\%(cauda epididymis) & $60.00 \pm 4.43$ & $68.75 \pm 6.84$ & $62.15 \pm 5.53$ & $42.40 \pm 2.02^{*}$ \\
$\quad$ Sperm (10\%/g cauda epididymis) & $402 \pm 29$ & $443 \pm 46$ & $400 \pm 32$ & $283 \pm 13^{*}$ \\
\hline
\end{tabular}

*Significantly different $(\mathrm{P} \leq 0.05)$ from the control group by Shirley's or Dunn's test.

**Significantly different $(\mathrm{P} \leq 0.01)$ from the control group by Williams' test.

${ }^{\text {a }}$ Data are presented as mean \pm standard error. Differences from the control group are not significant by Dunnett's test (tissue weights) or Dunn's test (spermatid measurements and sperm motility).

Incidences of clear cell focus in the liver were significantly increased in male rats exposed to $1,000 \mathrm{ppm}$; a slight increase was also observed in females at 1,000 ppm (Table 8, Table A-1, and Table A-2). The incidences of hepatocyte cytoplasmic vacuolization were significantly increased in 300 and 1,000 ppm males and in 1,000 ppm females; the severity of this lesion was increased in 1,000 ppm males.

Clear cell foci were scattered throughout the section and were characterized by enlarged hepatocytes with clear cytoplasm, particularly in the perinuclear region, but often involving the entire cell. The increased size of the hepatocytes occasionally resulted in minimal compression at the margin of the lesion. Hepatocyte cytoplasmic vacuolization was characterized by individual or clusters of large hepatocytes with pale cytoplasm and indistinct or microvesicular cytoplasmic vacuoles scattered throughout the section.

Incidences of hematopoietic cell proliferation in the spleen were significantly increased in 300 and 1,000 ppm males and in 1,000 ppm females (Table 8, Table A-1, and Table A-2). Incidences of splenic congestion were significantly increased in $1,000 \mathrm{ppm}$ males and in most exposed groups of females. Pigmentation occurred in the spleen of every rat in the study, but the severity of the lesion was increased in 300 and 1,000 ppm males and all exposed groups of females. 
Table 8. Incidences of Selected Nonneoplastic Lesions in Rats in the Three-month Drinking Water Study of $o$-Chloropyridine

\begin{tabular}{|c|c|c|c|c|c|c|}
\hline & 0 ppm & 10 ppm & 30 ppm & 100 ppm & 300 ppm & $1,000 \mathrm{ppm}$ \\
\hline \multicolumn{7}{|l|}{ Male } \\
\hline Liver $^{\mathrm{a}}$ & 10 & 10 & 10 & 10 & 10 & 10 \\
\hline Clear Cell Focus ${ }^{\mathrm{b}}$ & 0 & 0 & 0 & 0 & 0 & $6^{* *}$ \\
\hline $\begin{array}{l}\text { Hepatocyte, Vacuolization } \\
\text { Cytoplasmic }\end{array}$ & 0 & 0 & 0 & 0 & $6^{* *}(1.0)^{\mathrm{c}}$ & $9 * *(1.8)$ \\
\hline Spleen & 10 & 10 & 10 & 10 & 10 & 10 \\
\hline $\begin{array}{l}\text { Hematopoietic Cell } \\
\text { Proliferation }\end{array}$ & $4(1.8)$ & $8(2.0)$ & $6(1.8)$ & $6(1.7)$ & $10 * *(2.0)$ & $10 * *(2.0)$ \\
\hline Congestion & $4(1.0)$ & $1(1.0)$ & $2(1.0)$ & $0 *$ & $7(1.0)$ & $10 * *(1.0)$ \\
\hline Pigmentation & $10(1.0)$ & $10(1.0)$ & $10(1.3)$ & $10(1.0)$ & $10(2.0)$ & $10(2.0)$ \\
\hline Bone Marrow & 10 & 10 & 10 & 10 & 10 & 10 \\
\hline Hyperplasia & $1(1.0)$ & 0 & $2(1.0)$ & $1(1.0)$ & $9 * *(1.0)$ & $10 * *(1.9)$ \\
\hline Kidney & 10 & 10 & 10 & 10 & 10 & 10 \\
\hline $\begin{array}{l}\text { Renal Tubule, } \\
\text { Accumulation, Hyaline } \\
\text { Droplet }\end{array}$ & $10(2.1)$ & $10(2.4)$ & $10(2.3)$ & $9(1.8)$ & $5^{*}(1.2)$ & $0 * *$ \\
\hline \multicolumn{7}{|l|}{ Female } \\
\hline Liver & 10 & 10 & 10 & 10 & 10 & 10 \\
\hline Clear Cell Focus & 0 & 0 & 0 & 0 & 0 & 2 \\
\hline $\begin{array}{l}\text { Hepatocyte, Vacuolization } \\
\text { Cytoplasmic }\end{array}$ & 0 & 0 & 0 & 0 & 0 & $9 * *(1.3)$ \\
\hline Spleen & 10 & 10 & 10 & 10 & 10 & 10 \\
\hline $\begin{array}{l}\text { Hematopoietic Cell } \\
\text { Proliferation }\end{array}$ & $3(2.0)$ & $3(1.3)$ & $6(1.5)$ & $3(1.7)$ & $7(1.7)$ & $10 * *(1.9)$ \\
\hline Congestion & $1(2.0)$ & $7 * *(1.3)$ & $5(1.6)$ & $6^{*}(1.3)$ & $6^{*}(1.0)$ & $10 * *(1.8)$ \\
\hline Pigmentation & $10(2.0)$ & $10(2.2)$ & $10(2.3)$ & $10(2.4)$ & $10(2.2)$ & $10(3.0)$ \\
\hline Bone Marrow & 10 & 10 & 10 & 10 & 10 & 10 \\
\hline Hyperplasia & 0 & 0 & 0 & 0 & $3(1.0)$ & $9 * *(1.1)$ \\
\hline $\begin{array}{l}{ }^{*} \text { Significantly different }(\mathrm{P} \leq 0.0 \\
{ }^{* *} \mathrm{P} \leq 0.01 \text {. } \\
\text { aNumber of animals with tissue } \\
\text { bNumber of animals with lesion } \\
{ }^{\mathrm{c}} \text { Average severity grade of lesio }\end{array}$ & $\begin{array}{l}\text { from the co } \\
\text { amined mic } \\
\text { in affected }\end{array}$ & $\begin{array}{l}1 \text { group by th } \\
\text { opically. } \\
\text { nals: } 1=\text { min }\end{array}$ & \multicolumn{3}{|c|}{$\begin{array}{l}\text { aNumber of animals with tissue examined microscopically. } \\
\text { bNumber of animals with lesion. }\end{array}$} & \\
\hline \multicolumn{7}{|c|}{$\begin{array}{l}\text { Splenic hematopoietic cell proliferation was characterized by an increase of primarily erythroid } \\
\text { precursors in the sinusoids of the spleen above background levels. Severity grades were } \\
\text { evaluated semiquantitatively and ranged from a minimal to mild increase in cellularity. } \\
\text { Background hematopoietic cell presence in control animals was fairly minimal although all } \\
\text { animals had at least some hematopoietic precursors in the spleen characterized by scattered } \\
\text { individual and small clusters of hematopoietic cells throughout the red pulp. The control males } \\
\text { had a slightly higher background level of hematopoietic cells than the control females and } \\
\text { therefore the severity grade of hematopoietic cell proliferation was judged relative to the }\end{array}$} \\
\hline
\end{tabular}


appropriate sex-matched control. Minimal hematopoietic proliferation consisted of $10 \%$ to $20 \%$ increased numbers of small clusters of hematopoietic cells in the red pulp compared to the majority of the controls. Mild hematopoietic cell proliferation was characterized by an approximately $21 \%$ to $50 \%$ increase in numbers of small clusters throughout the red pulp, but without confluence of clusters of hematopoietic cells. As stated above, background hematopoiesis in controls was not particularly prominent and therefore a minimal to mild increase in hematopoietic cells still did not result in loss of architecture or impingement on the white pulp elements.

Splenic congestion was characterized by an increased amount of blood in the red pulp of the spleen compared to controls. Severity of congestion was assessed semiquantitatively by the numbers (density) of red cells in the red pulp and varied from minimal to mild. As the numbers of red cells increased, less space was noted around individual red blood cells and the overall color of the spleen became more brightly eosinophilic. Minimal congestion had approximately $10 \%$ to $20 \%$ more red blood cells than controls and was best appreciated in the subcapsular red pulp. Mild congestion had $21 \%$ to $35 \%$ more red cells in sinusoids than controls, particularly in the subcapsular areas at the lateral edges of the spleen as seen on cross section, and also in red pulp in the central areas of the spleen. However, with both minimal and mild congestion, the overall architecture of the spleen was retained.

Pigmentation in the spleen was characterized by an increase in gold-colored pigment within macrophages. It is suspected that this pigmentation represents hemosiderin. Severity of pigmentation was assessed semiquantitatively and a threshold was not utilized. All spleens had at least some pigmentation with increasing severity in both the number of macrophages that contained pigment and in the amount of pigment within the macrophages. Minimal pigmentation was characterized by a faint gold pigment in $20 \%$ to $40 \%$ of individual macrophages scattered throughout the red pulp. Mild pigmentation was characterized by an approximately $50 \%$ increase in the amount of pigment in individual macrophages so that the cytoplasm was a more intense gold color. Also, mild pigmentation was characterized by an increased number of pigmentcontaining macrophages with at least $50 \%$ of macrophages containing pigment; occasional clusters of macrophages with distended cytoplasm containing pigment were noted. Moderate pigmentation had increased numbers of macrophages containing pigment and greater than $80 \%$ of macrophages had at least some pigmented material in the cytoplasm. Clusters of macrophages, containing abundant pigment distending the cytoplasm, were more numerous in spleens with moderate pigmentation than in spleens with mild pigmentation.

The incidences of bone marrow hyperplasia were significantly increased in 300 and 1,000 ppm males and 1,000 ppm females (Table 8, Table A-1, and Table A-2). Bone marrow hyperplasia was evaluated for overall cellularity of hematopoietic cells (myeloid, erythroid, and megakaryocytic cell lines). The cellularity was assessed semiquantitatively as a percentage of hematopoietic cells to fat in the marrow of the femur. In control males, the bone marrow was approximately $20 \%$ hematopoietic cells and $80 \%$ fat. In males, minimal hyperplasia was characterized by $21 \%$ to $40 \%$ hematopoietic cells and mild hyperplasia was diagnosed when $41 \%$ to $60 \%$ of the marrow was composed of hematopoietic cells. Control females had a higher percentage of fat in the marrow than male controls with approximately $10 \%$ hematopoietic cells and $90 \%$ fat. Minimal hyperplasia was characterized by approximately $11 \%$ to $20 \%$ hematopoietic cells and $80 \%$ fat. Mild hyperplasia was diagnosed when the marrow contained approximately $21 \%$ to $50 \%$ hematopoietic cells. Bone marrow hyperplasia was characterized by 
an increase in erythroid and myeloid cell lines with all maturation stages represented. Numbers of megakaryocytes also increased with increasing bone marrow cellularity, and mast cells tended to increase with increasing cellularity. The myeloid/erythroid ratio was maintained between 1:1 and 2.5:1 across all groups, although there was a slight relative increase in the erythroid cells.

Significant decreases in the incidences of renal tubule hyaline droplet accumulation in the kidney occurred in 300 and 1,000 ppm males (Table 8 and Table A-1). Hyaline droplets are normal lysosomal accumulations of $\alpha 2 \mathrm{u}$-globulin, a protein specific to male rats, from the glomerular filtrate. This eosinophilic accumulation normally occurs within the cytoplasm of proximal convoluted tubules in the renal cortex.

\section{Mice}

\section{Two-week Dermal Study}

All mice survived to the end of the study (Table 9). The final mean body weights and body weight gains of all dosed groups were similar to those of the vehicle control groups. There were no clinical findings related to $o$-chloropyridine administration.

Relative right kidney weights of 50 and $100 \mathrm{mg} / \mathrm{kg}$ males and $25 \mathrm{mg} / \mathrm{kg}$ females were significantly greater than those of the vehicle controls (Table 10 and Table C-3). No gross or microscopic lesions were considered related to $o$-chloropyridine administration.

Exposure concentration selection rationale: Exposure concentrations for the 3-month drinking water study were selected for comparison to the 3 -month pyridine study results ${ }^{33}$.

Table 9. Survival and Body Weights of Mice in the Two-week Dermal Study of $o$-Chloropyridine ${ }^{\text {a }}$

\begin{tabular}{cccccc}
\hline Dose (mg/kg) & Survival $^{\mathbf{b}}$ & $\begin{array}{c}\text { Initial Body } \\
\text { Weight }(\mathbf{g})\end{array}$ & $\begin{array}{c}\text { Final Body } \\
\text { Weight }(\mathbf{g})\end{array}$ & $\begin{array}{c}\text { Change in Body } \\
\text { Weight (g) }\end{array}$ & $\begin{array}{c}\text { Final Weight } \\
\text { Relative to } \\
\text { Controls (\%) }\end{array}$ \\
\hline Male & & & & & \\
0 & $5 / 5$ & $23.7 \pm 0.3$ & $25.7 \pm 0.3$ & $2.0 \pm 0.3$ & \\
6.25 & $5 / 5$ & $23.3 \pm 0.7$ & $26.4 \pm 0.5$ & $3.1 \pm 0.4$ & 103 \\
12.5 & $5 / 5$ & $23.5 \pm 0.6$ & $26.8 \pm 0.4$ & $3.3 \pm 0.6$ & 104 \\
25 & $5 / 5$ & $23.6 \pm 0.7$ & $26.1 \pm 0.5$ & $2.5 \pm 0.4$ & 102 \\
50 & $5 / 5$ & $23.5 \pm 0.5$ & $25.7 \pm 0.3$ & $2.2 \pm 0.2$ & 100 \\
100 & $5 / 5$ & $23.1 \pm 0.9$ & $25.6 \pm 0.7$ & $2.5 \pm 0.3$ & 100 \\
\hline Female & & & & & \\
0 & $5 / 5$ & $19.9 \pm 0.7$ & $23.2 \pm 0.6$ & $3.3 \pm 0.2$ & 100 \\
6.25 & $5 / 5$ & $19.5 \pm 0.6$ & $23.1 \pm 0.4$ & $3.6 \pm 0.3$ & 98 \\
12.5 & $5 / 5$ & $19.8 \pm 0.6$ & $22.8 \pm 0.6$ & $3.0 \pm 0.3$ & 99 \\
25 & $5 / 5$ & $19.8 \pm 0.6$ & $23.0 \pm 0.6$ & $3.2 \pm 0.2$ & 98 \\
50 & $5 / 5$ & $19.9 \pm 0.7$ & $22.7 \pm 0.6$ & $2.8 \pm 0.4$ & 100 \\
\hline 100 & $5 / 5$ & $20.0 \pm 0.4$ & $23.3 \pm 0.4$ & $3.3 \pm 0.2$ & \\
\hline
\end{tabular}

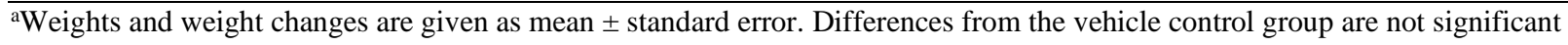
by Dunnett's test.

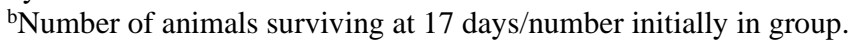


Table 10. Kidney Weights and Kidney-Weight-to-Body-Weight Ratios for Mice in the Two-week Dermal Study of $o$-Chloropyridine ${ }^{a}$

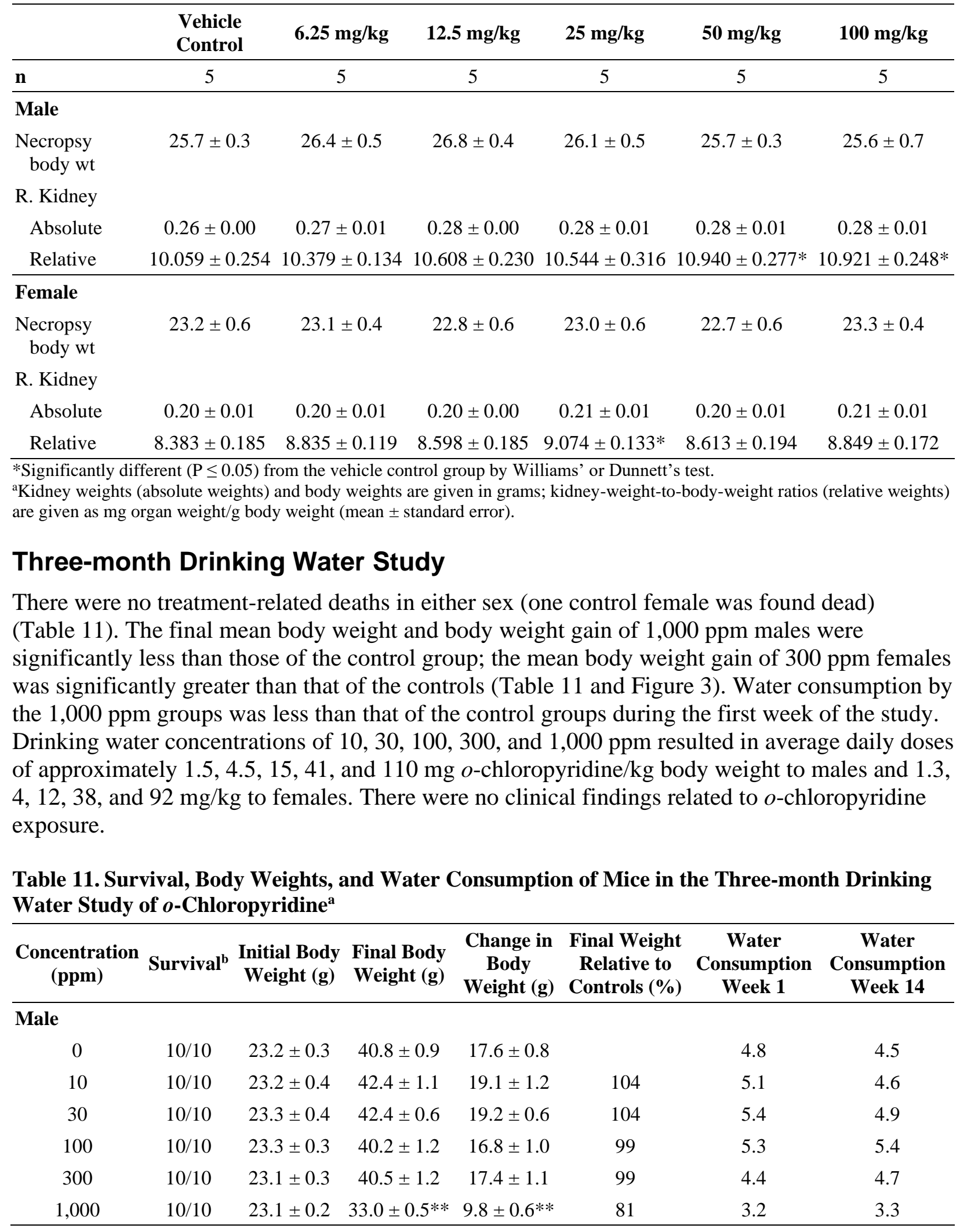




\begin{tabular}{cccccccc}
\hline $\begin{array}{c}\text { Concentration } \\
(\mathbf{p p m})\end{array}$ & Survival $^{\mathbf{b}}$ & $\begin{array}{c}\text { Initial Body Final Body } \\
\text { Weight (g) }\end{array}$ & $\begin{array}{c}\text { Change in } \\
\text { Wody }(\mathbf{g})\end{array}$ & $\begin{array}{c}\text { Final Weight } \\
\text { Weight (g) }\end{array}$ & $\begin{array}{c}\text { Water } \\
\text { Controls (\%) }\end{array}$ & $\begin{array}{c}\text { Water } \\
\text { Consumption } \\
\text { Week 1 }\end{array}$ & $\begin{array}{c}\text { Consumption } \\
\text { Week 14 }\end{array}$ \\
\hline Female & & & & & & & \\
0 & $9 / 10^{\mathrm{c}}$ & $18.6 \pm 0.4$ & $28.5 \pm 1.4$ & $9.7 \pm 1.2$ & & 2.8 & 3.5 \\
10 & $10 / 10$ & $19.1 \pm 0.3$ & $31.8 \pm 1.0$ & $12.8 \pm 1.0$ & 112 & 3.0 & 3.3 \\
30 & $10 / 10$ & $18.8 \pm 0.3$ & $31.2 \pm 1.3$ & $12.4 \pm 1.1$ & 109 & 2.7 & 3.4 \\
100 & $10 / 10$ & $19.0 \pm 0.3$ & $30.3 \pm 1.1$ & $11.3 \pm 0.9$ & 106 & 2.3 & 3.4 \\
300 & $10 / 10$ & $18.1 \pm 0.5$ & $31.9 \pm 0.8$ & $13.8 \pm 0.7 *$ & 112 & 3.3 & 3.4 \\
1,000 & $10 / 10$ & $18.6 \pm 0.4$ & $28.4 \pm 0.6$ & $9.9 \pm 0.5$ & 100 & 1.6 & 2.8 \\
\hline
\end{tabular}

*Significantly different $(\mathrm{P} \leq 0.05)$ from the control group by Dunnett's test.

**Significantly different $(\mathrm{P} \leq 0.01)$ from the control group by Williams' test.

${ }^{a}$ Weights and weight changes are given as mean \pm standard error. Water consumption is expressed as grams per animal per day.

${ }^{b}$ Number of animals surviving at 14 weeks/number initially in group.

${ }^{\mathrm{c}}$ Week of death: 11 . 

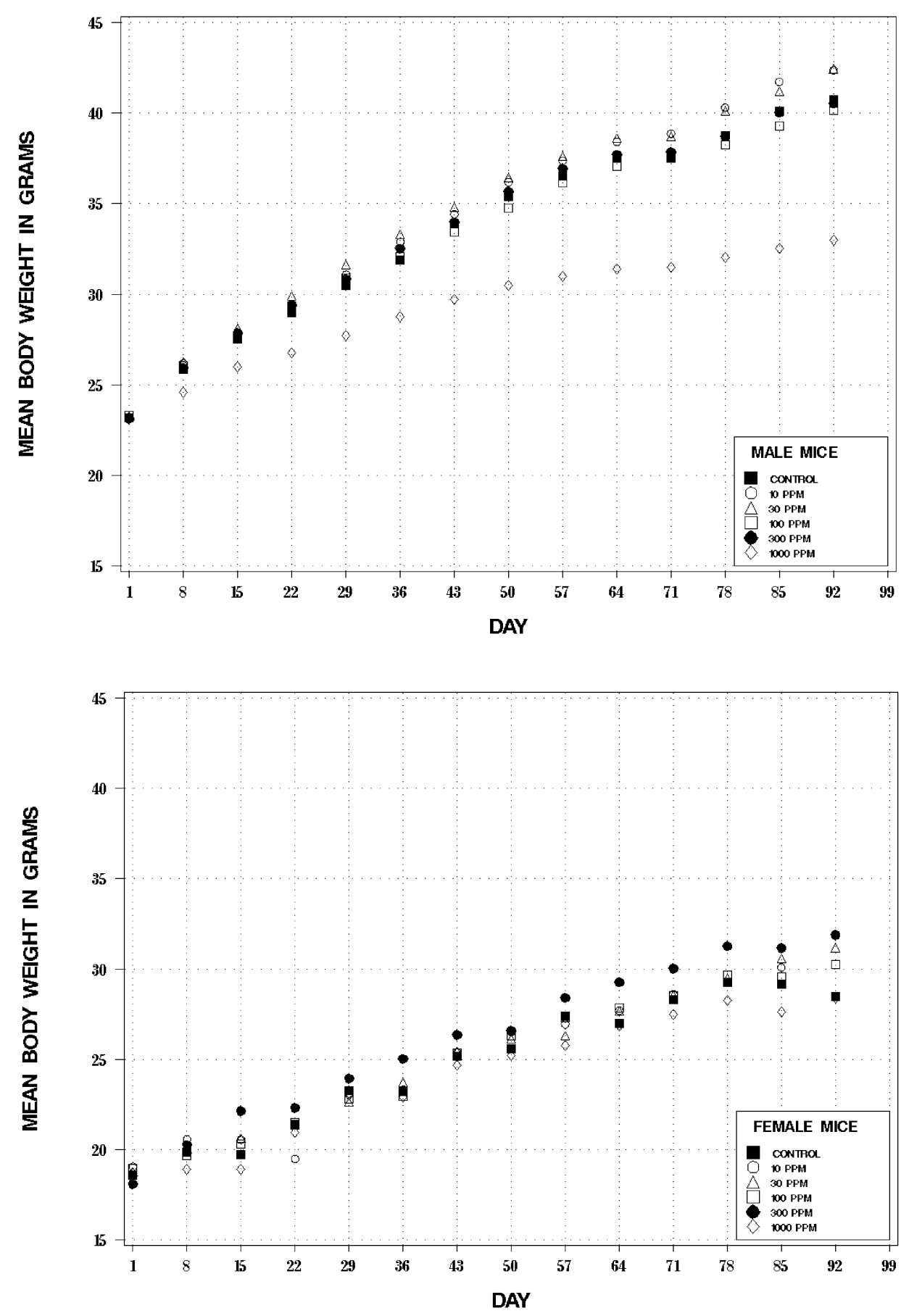

Figure 3. Growth Curves for Mice Exposed to o-Chloropyridine in Drinking Water for Three Months

The hematology data for mice are presented in Table 12 and Table B-2. Similar to what occurred in the rat study, at week 14, a decreased erythron occurred; however, this was only observed in the $1,000 \mathrm{ppm}$ females. The erythron decrease was small $(<10 \%)$ and was demonstrated by decreases in hematocrit value, hemoglobin concentration and erythrocyte count. As with the rats, 
the erythron decrease was accompanied by a small reduction (approximately $3 \%$ ) in erythrocyte size, evidenced by a decrease in the mean cell volume.

The absolute and relative liver weights of all exposed groups of males and of 300 (absolute, $32.8 \%$ ) and 1,000 ppm (absolute, 59\%) females were significantly greater than those of the control groups (Table 13 and Table $\mathrm{C}-4)$. The absolute (15.8\%) and relative right kidney weights of $1,000 \mathrm{ppm}$ females were significantly greater than those of the controls. There were no significant differences in any of the reproductive organ weights or sperm parameters of males, or in the estrous cyclicity of females, at any exposure concentration tested when compared to the control groups (Table D-4, Table D-5, and Table D-6 and Figure D-2).

Table 12. Selected Hematology Data for Mice in the Three-month Drinking Water Study of $o$-Chloropyridine ${ }^{\mathrm{a}}$

\begin{tabular}{lcccccc}
\hline & $\mathbf{0 ~} \mathbf{~ p p m}$ & $\mathbf{1 0} \mathbf{~ p p m}$ & $\mathbf{3 0} \mathbf{~ p p m}$ & $\mathbf{1 0 0} \mathbf{~ p p m}$ & $\mathbf{3 0 0} \mathbf{~ p p m}$ & $\mathbf{1 , 0 0 0} \mathbf{~ p p m}$ \\
\hline Male & & & & & & \\
$\mathbf{n}$ & 10 & 10 & 10 & 10 & 10 & 10 \\
\hline Hematocrit $(\%)$ & $51.2 \pm 0.4$ & $51.4 \pm 0.5$ & $50.4 \pm 0.7$ & $50.2 \pm 0.4$ & $50.3 \pm 0.6$ & $50.5 \pm 0.6$ \\
Hemoglobin $(\mathrm{g} / \mathrm{dL})$ & $16.4 \pm 0.1$ & $16.5 \pm 0.1$ & $16.1 \pm 0.2$ & $16.1 \pm 0.1$ & $16.2 \pm 0.2$ & $16.2 \pm 0.2$ \\
Erythrocytes $\left(10^{6} / \mu \mathrm{L}\right)$ & $10.63 \pm 0.09$ & $10.78 \pm 0.10$ & $10.46 \pm 0.13$ & $10.65 \pm 0.07$ & $10.67 \pm 0.11$ & $10.69 \pm 0.08$ \\
Reticulocytes $\left(10^{6} / \mu \mathrm{L}\right)$ & $0.25 \pm 0.02$ & $0.27 \pm 0.02$ & $0.29 \pm 0.03$ & $0.28 \pm 0.02$ & $0.28 \pm 0.03$ & $0.26 \pm 0.03$ \\
Mean cell volume $(\mathrm{fL})$ & $47.3 \pm 0.3$ & $47.3 \pm 0.3$ & $47.5 \pm 0.2$ & $46.8 \pm 0.1$ & $47.0 \pm 0.4$ & $46.7 \pm 0.2$ \\
Mean cell hemoglobin $(\mathrm{pg})$ & $15.2 \pm 0.1$ & $15.2 \pm 0.1$ & $15.2 \pm 0.1$ & $15.1 \pm 0.1$ & $15.1 \pm 0.1$ & $14.9 \pm 0.1$ \\
Mean cell hemoglobin & $32.1 \pm 0.2$ & $32.1 \pm 0.1$ & $32.0 \pm 0.1$ & $32.2 \pm 0.1$ & $32.2 \pm 0.1$ & $32.1 \pm 0.1$ \\
$\quad$ concentration $(\mathrm{g} / \mathrm{dL})$ & & & & & & \\
\hline
\end{tabular}

\section{Female}

\begin{tabular}{lcccccc}
$\mathbf{n}$ & 9 & 10 & 10 & 10 & 10 & 10 \\
\hline Hematocrit $(\%)$ & $50.9 \pm 1.1$ & $50.5 \pm 0.7$ & $48.9 \pm 0.4$ & $50.5 \pm 0.4$ & $48.8 \pm 0.6$ & $46.6 \pm 0.4^{* *}$ \\
Hemoglobin $(\mathrm{g} / \mathrm{dL})$ & $16.2 \pm 0.3$ & $16.0 \pm 0.2$ & $15.7 \pm 0.1$ & $16.2 \pm 0.1$ & $15.7 \pm 0.2$ & $15.1 \pm 0.1^{* *}$ \\
Erythrocytes $\left(10^{6} / \mu \mathrm{L}\right)$ & $10.56 \pm 0.20$ & $10.42 \pm 0.12$ & $10.23 \pm 0.09$ & $10.54 \pm 0.07$ & $10.22 \pm 0.13$ & $9.96 \pm 0.09 * *$ \\
Reticulocytes $\left(10^{6} / \mu \mathrm{L}\right)$ & $0.40 \pm 0.03$ & $0.33 \pm 0.03$ & $0.32 \pm 0.03$ & $0.37 \pm 0.03$ & $0.37 \pm 0.03$ & $0.31 \pm 0.03$ \\
Mean cell volume $(\mathrm{fL})$ & $48.2 \pm 0.2$ & $48.2 \pm 0.1$ & $47.9 \pm 0.2$ & $47.9 \pm 0.2$ & $47.7 \pm 0.2$ & $46.9 \pm 0.2^{* *}$ \\
Mean cell hemoglobin $(\mathrm{pg})$ & $15.3 \pm 0.1$ & $15.3 \pm 0.0$ & $15.3 \pm 0.1$ & $15.4 \pm 0.1$ & $15.4 \pm 0.0$ & $15.2 \pm 0.1$ \\
$\begin{array}{l}\text { Mean cell hemoglobin } \\
\text { concentration }(\mathrm{g} / \mathrm{dL})\end{array}$ & $31.8 \pm 0.1$ & $31.7 \pm 0.1$ & $32.0 \pm 0.1$ & $32.0 \pm 0.1$ & $32.2 \pm 0.1$ & $32.4 \pm 0.1^{* *}$ \\
\hline
\end{tabular}

**Significantly different $(\mathrm{P} \leq 0.01)$ from the control group by Shirley's test.

a Data are presented as mean \pm standard error. Statistical tests were performed on unrounded data. 
Table 13. Selected Organ Weights and Organ-Weight-to-Body-Weight Ratios for Mice in the Three-month Drinking Water Study of $o$-Chloropyridine ${ }^{a}$

\begin{tabular}{lcccccc}
\hline & $\mathbf{0 ~} \mathbf{~ p m}$ & $\mathbf{1 0} \mathbf{~ p p m}$ & $\mathbf{3 0} \mathbf{~ p p m}$ & $\mathbf{1 0 0} \mathbf{~ p p m}$ & $\mathbf{3 0 0} \mathbf{~ p p m}$ & $\mathbf{1 , 0 0 0} \mathbf{~ p p m}$ \\
\hline $\begin{array}{l}\text { Male } \\
\mathbf{n}\end{array}$ & 10 & 10 & 10 & 10 & 10 & 10 \\
\hline $\begin{array}{l}\text { Necropsy } \\
\text { body wt }\end{array}$ & $40.8 \pm 0.9$ & $42.4 \pm 1.1$ & $42.4 \pm 0.6$ & $40.2 \pm 1.2$ & $40.5 \pm 1.2$ & $33.0 \pm 0.5^{* *}$ \\
R. Kidney & & & & & & \\
$\quad$ Absolute & $0.34 \pm 0.01$ & $0.35 \pm 0.01$ & $0.35 \pm 0.01$ & $0.35 \pm 0.01$ & $0.35 \pm 0.01$ & $0.31 \pm 0.01$ \\
$\quad$ Relative & $8.237 \pm 0.218$ & $8.203 \pm 0.090$ & $8.193 \pm 0.244$ & $8.631 \pm 0.217$ & $8.556 \pm 0.229$ & $9.242 \pm 0.369^{*}$ \\
Liver & & & & & \\
$\quad$ Absolute & $1.70 \pm 0.06$ & $1.89 \pm 0.07 *$ & $1.95 \pm 0.04 *$ & $1.84 \pm 0.07 *$ & $2.01 \pm 0.08^{* *}$ & $1.95 \pm 0.05^{* *}$ \\
Relative & $41.624 \pm 0.778$ & $44.531 \pm 0.718^{*}$ & $45.851 \pm 0.819^{* *}$ & $45.766 \pm 0.974 * *$ & $49.467 \pm 0.796^{* *}$ & $59.121 \pm 0.830^{* *}$ \\
\hline
\end{tabular}

Female

\begin{tabular}{ccccccc}
$\mathbf{n}$ & 9 & 10 & 10 & 10 & 10 & 10 \\
\hline $\begin{array}{c}\text { Necropsy } \\
\text { body wt }\end{array}$ & $28.5 \pm 1.4$ & $31.8 \pm 1.0$ & $31.2 \pm 1.3$ & $30.3 \pm 1.1$ & $31.9 \pm 0.8$ & $28.4 \pm 0.6$ \\
$\begin{array}{c}\text { R. Kidney } \\
\text { Absolute }\end{array}$ & $0.19 \pm 0.01$ & $0.20 \pm 0.00$ & $0.19 \pm 0.01$ & $0.19 \pm 0.01$ & $0.20 \pm 0.00$ & $0.22 \pm 0.01^{* *}$ \\
Relative & $6.866 \pm 0.352$ & $6.203 \pm 0.206$ & $6.212 \pm 0.170$ & $6.232 \pm 0.174$ & $6.321 \pm 0.154$ & $7.676 \pm 0.156^{*}$
\end{tabular}

Liver

$\begin{array}{lcccccc}\text { Absolute } & 1.22 \pm 0.09 & 1.37 \pm 0.04 & 1.34 \pm 0.05 & 1.35 \pm 0.07 & 1.62 \pm 0.05 * * & 1.94 \pm 0.0 .05^{* *} \\ \text { Relative } & 42.778 \pm 1.560 & 43.065 \pm 1.066 & 43.266 \pm 0.645 & 44.466 \pm 1.122 & 50.818 \pm 1.290^{* *} & 68.154 \pm 1.026^{* *}\end{array}$

*Significantly different $(\mathrm{P} \leq 0.05)$ from the control group by Williams' or Dunnett's test.

$* * \mathrm{P} \leq 0.01$

${ }^{\text {a } O r g a n ~ w e i g h t s ~(a b s o l u t e ~ w e i g h t s) ~ a n d ~ b o d y ~ w e i g h t s ~ a r e ~ g i v e n ~ i n ~ g r a m s ; ~ o r g a n-w e i g h t-t o-b o d y-w e i g h t ~ r a t i o s ~(r e l a t i v e ~ w e i g h t s) ~}$ are given as $\mathrm{mg}$ organ weight/g body weight (mean \pm standard error).

Incidences of hepatocyte centrilobular hypertrophy occurred with positive trends in exposed groups of males and females, and the incidences were significantly increased in the 300 and 1,000 ppm groups compared to those in the controls (Table 14, Table A-3, and Table A-4); the severity of this lesion also increased with exposure concentration. The lesion was characterized by minimal to mild enlargement (hypertrophy) of hepatocytes that were centrilobular in distribution. Hypertrophic hepatocytes had homogeneous or slightly granular eosinophilic cytoplasm. The nuclei were often enlarged and contained prominent basophilic chromatin aggregates. 
Table 14. Incidences of Hepatocyte Centrilobular Hypertrophy in the Liver of Mice in the Three-month Drinking Water Study of $o$-Chloropyridine

\begin{tabular}{|c|c|c|c|c|c|c|}
\hline & 0 ppm & 10 ppm & 30 ppm & 100 ppm & 300 ppm & 1,000 ppm \\
\hline \multicolumn{7}{|l|}{ Male } \\
\hline $\begin{array}{l}\text { Number Examined } \\
\text { Microscopically }\end{array}$ & 10 & 10 & 10 & 10 & 10 & 10 \\
\hline $\begin{array}{l}\text { Centrilobular, Hepatocyte, } \\
\text { Hypertrophy }\end{array}$ & 0 & 0 & 0 & $1(1.0)^{\mathrm{b}}$ & $6^{* *}(1.5)$ & $9 * *(2.1)$ \\
\hline \multicolumn{7}{|l|}{ Female } \\
\hline $\begin{array}{l}\text { Number Examined } \\
\text { Microscopically }\end{array}$ & 10 & 10 & 10 & 10 & 10 & 10 \\
\hline $\begin{array}{l}\text { Centrilobular, Hepatocyte, } \\
\text { Hypertrophy }\end{array}$ & 0 & 0 & 0 & 0 & $4 *(1.0)$ & $10 * *(1.5)$ \\
\hline
\end{tabular}

\section{Absorption, Distribution, Metabolism, Excretion, and Toxicokinetics}

The metabolism and disposition of $o$-chloropyridine was investigated following a single oral, intravenous, or intraperitoneal dose in male F344 rats (Appendix J). For all studies, formulations were prepared by addition of Alkamuls ${ }^{\circledR}$ EL-620/L (no more than $15 \%$ of the dose) (Rhodia, Inc., Cranbury, NJ) to known amounts of $o$-chloropyridine following which distilled deionized water and the appropriate amount of $\left[{ }^{14} \mathrm{C}\right]$-o-chloropyridine were added. Dosing volumes were either $5 \mathrm{~mL} / \mathrm{kg}$ (oral gavage and intraperitoneal injection) or $1 \mathrm{~mL} / \mathrm{kg}$ (intravenous injection). Oral doses were administered by intragastric gavage using a syringe equipped with a 16-gauge gavage needle. Intravenous doses were injected into a lateral tail vein using a syringe equipped with a 27 -gauge needle.

Following gavage administration of 0.1 or $10 \mathrm{mg}\left[{ }^{14} \mathrm{C}\right]-O$-chloropyridine $/ \mathrm{kg}$, there was no dose-related difference in the disposition. Approximately $82 \%$ of the administered dose was recovered by 72 hours following administration. The absorption was rapid with maximum blood concentration being reached within 30 minutes following administration. The distribution $\left(\mathrm{t}_{1 / 2 \alpha}\right)$ and elimination $\left(\mathrm{t}_{1 / 2 \beta}\right)$ half-lives, estimated based on the measurement of the total radioactivity in blood were 1.14 hours and 46 hours, respectively. Excretion in urine accounted for approximately $36 \%$ to $39 \%$ and that in feces about $28 \%$. The observation that $27 \%$ of a $50 \mathrm{mg} / \mathrm{kg}$ intraperitoneal dose was excreted in bile in rats by 4 hours after administration suggests that the high fecal excretion following gavage administration was likely due to biliary excretion and not due to poor absorption. Approximately $11 \%$ to $13 \%$ of the gavage dose was recovered as $\mathrm{CO}_{2}$ and approximately $1 \%$ was eliminated in breath as volatile organics. The total radioactivity in tissue at 72 hours following administration was approximately 3\% to $4 \%$ with the highest tissue:blood ratios observed in the liver and kidney. Profiling of urine showed that the compound is readily metabolized. Three major metabolites carrying most of the radioactivity were found to contain disubstituted pyridine structures, one of which was identified as 2-chloro-5-hydroxypyridine. 
Following intravenous administration of $1 \mathrm{mg} / \mathrm{kg}\left[{ }^{14} \mathrm{C}\right]-o$-chloropyridine in male $\mathrm{F} 344 / \mathrm{N}$ rats, the distribution $\left(\mathrm{T}_{1 / 2 \alpha}\right)$ and elimination $\left(\mathrm{T}_{1 / 2 \beta}\right)$ half-lives were 0.103 hours and 1.04 hours, respectively, based on the measurement of parent compound (Appendix J).

\section{Genetic Toxicology}

$o$-Chloropyridine (10 to10,000 $\mu \mathrm{g} / \mathrm{plate}$ ) was mutagenic in Salmonella typhimurium strains TA98 and TA100 when tested with induced rat or hamster liver S9 metabolic activation enzymes; no mutagenic activity was observed in either strain in the absence of metabolic activation (Table E-1). In both TA98 and TA100, the mutagenic activity observed with $o$-chloropyridine was stronger with hamster S9 (higher magnitude response observed at lower concentrations of $o$-chloropyridine). In vivo, no increases in the frequencies of micronucleated normochromatic erythrocytes were observed in peripheral blood samples from male or female mice exposed to $o$-chloropyridine (10 to 1,000 ppm) in drinking water for 3 months (Table E-2). In addition, no significant exposure concentration-related changes in the percentage of immature (polychromatic) erythrocytes were observed, suggesting that exposure to $o$-chloropyridine did not alter the process of erythropoiesis in the bone marrow of mice. 


\section{Discussion}

$o$-Chloropyridine is used as a key intermediate for the manufacturing of pyrithione-based chemicals used in cosmetics and agriculture. It is also a starting material in the production of certain antihistamines and antiarrhythmic pharmaceuticals. In 2006, the production and import volume of $o$-chloropyridine in the United States was reported to range from 1 million to 10 million pounds ${ }^{11}$. Occupational exposure can occur during the synthesis of $o$-chloropyridine and during its use as an industrial chemical intermediate. High vapor concentrations due to liquid spills have been detected in some industrial processing areas, suggesting the potential for significant exposure ${ }^{17}$. Environmental exposure to $o$-chloropyridine is also possible; it has been found as a trace organic contaminant in process streams and wastewater as well as some drinking water sources $^{20 ; 21}$. Despite the high production volume and potential for occupational and environmental exposure, there is little toxicity data available for this compound and no guidelines have been set by the National Institute for Occupational Safety and Health or the Occupational Safety and Health Administration. The current report describes the 2-week dermal and 3-month drinking water studies for $o$-chloropyridine administered to F344/N rats and $\mathrm{B} 6 \mathrm{C} 3 \mathrm{~F} 1 / \mathrm{N}$ mice. For the 3-month studies, the route of exposure was changed to drinking water, allowing for comparison of $o$-chloropyridine toxicity with the results of the NTP 3-month drinking water study of a structurally similar compound, pyridine ${ }^{33}$. Toxicity targets of $o$-chloropyridine were similar across species and routes of exposure, including increased liver weight with accompanying histologic changes in the liver for the 3-month studies.

In the 2-week dermal studies, all rats and mice survived until the end of the study with no significant changes in body weights. Some significant changes in liver and kidney weights were observed, with no supporting histologic changes.

In the 3-month drinking water studies with $o$-chloropyridine, there was no effect on survival in rats or mice. The major findings in rats related to a possible inefficient erythropoiesis, evidenced by changes in erythron, bone marrow, and spleen. In mice, the major finding was greater liver weights, compared to controls, accompanied by centrilobular hepatocyte hypertrophy.

In the current 3-month drinking water study, significant changes, both increases and decreases, in hematology values were observed in male rats. On day 4, hematology results demonstrated an increase in circulating erythron (indicated by hematocrit, hemoglobin concentration, and erythrocytes) in 1,000 ppm male rats. This change was likely a physiologic response secondary to dehydration, due to a $50 \%$ decrease in water consumption. In addition, no changes in renal function were observed, indicated by creatinine concentration, supporting a dehydration hypothesis. Observations of exposure concentration-related and progressive hyperalbuminemia and hyperproteinemia should be considered a physiological response to dehydration and are unlikely to be a toxicologic response to $o$-chloropyridine.

While early changes in erythron were likely due to dehydration, by day 22, changes in hematocrit, hemoglobin, and erythrocytes were reversed, demonstrating a decrease in erythron that persisted until the end of the study. At week 14, an increase in reticulocyte count was also observed; these results together indicate an erythropoietic response that is further supported by increased incidences of hematopoietic cell proliferation and congestion in the spleen and hyperplasia of the bone marrow. The minimal microcytic, normochromic, and responsive 
erythron decreases observed on day 22 and at week 14 involved production of smaller erythrocytes and would indicate some mild exposure-related ineffective erythropoiesis potentially involving an alteration in iron availability/metabolism or the production or availability of heme or hemoglobin. Classically, microcytic erythron decreases have been associated with an iron deficiency ${ }^{49}$. Because there was no clinical evidence of hemorrhage or blood loss, however, the microcytic change would suggest an ineffective erythropoiesis related to some alteration in iron and/or heme/hemoglobin metabolism. Such microcytic-type erythron decreases have been observed in rats with multiple agent types [e.g., lead ${ }^{50}$; cupric sulfate ${ }^{51}$; estragole $^{52}$ ]. In fact, in the 3-month study of pyridine, a similar iron deficiency-like response occurred in rats ${ }^{33}$. Mice demonstrated a similar decrease in erythron, although they appeared to be less sensitive, with effects seen only in high-dose females and without an observable erythropoietic response.

A decrease in the incidence and severity of hyaline droplet accumulation was observed in male rats in the 300 and 1,000 ppm groups. Hyaline droplets are normal lysosomal accumulations of $\alpha 2 \mathrm{u}$-globulin, a protein specific to male rats that is synthesized in the liver. Decreased synthesis of this protein by the liver may explain the decreased hyaline droplet accumulation that was observed. A study in rats treated with diethylnitrosamine reported a decrease in $\alpha 2 \mathrm{u}$-globulin mRNA in the liver, demonstrating that some chemicals are capable of affecting $\alpha 2 \mathrm{u}$-globulin synthesis ${ }^{53}$. In the present study, $\alpha 2 \mathrm{u}$-globulin mRNA was not measured, however decreased synthesis of the $\alpha 2 \mathrm{u}$-globulin protein is a potential explanation for the lower hyaline droplet accumulation that was observed in male rat kidney.

In humans, it has been reported that the pathology associated with $o$-chloropyridine is similar to that of pyridine exposure. Acute, low-dose exposures, that do not cause clinical symptoms, have been associated with centrilobular fatty degeneration, congestion, and cellular infiltration in the liver; low-dose chronic exposures may lead to cirrhosis ${ }^{4}$. In the current study, $o$-chloropyridine exposure in rats resulted in histopathologic lesions of the liver that may indicate a metabolic, adaptive response to treatment, including clear cell foci and hepatocyte cytoplasmic vacuolization. These changes were also observed in the 2-week dermal study with $o$-chloropyridine. While the liver was also a target in the pyridine 3-month drinking water study ${ }^{33}$, the lesions produced in the F344/N rat were of a different nature, suggesting direct toxicity (centrilobular degeneration, hypertrophy, inflammation, and pigmentation). In addition to pyridine, NTP also performed a 3-month drinking water study in F344/N rats with a structurally similar compound, $\beta$-picoline, which targeted the kidney, resulting in increased severity of $\alpha 2 \mathrm{u}$-globulin-mediated nephropathy and produced no liver lesions ${ }^{54}$. Large differences also exist in the bacterial mutagenicity of these three structurally similar compounds; $o$-chloropyridine is a potent bacterial mutagen while neither $\beta$-picoline nor pyridine is mutagenic.

Under the conditions of these 3-month drinking water studies, there were treatment-related organ weight changes and lesions in male and female rats and mice. The major target tissues in rats affected by $o$-chloropyridine exposure included the kidney, spleen, bone marrow, and liver; the major target organ in mice was the liver. The measurement most sensitive to $o$-chloropyridine exposure in male rats was increased absolute (all exposure groups) and relative (all exposure groups except $100 \mathrm{ppm}$ ) kidney weights in the absence of histopathologic changes [lowestobserved-effect level $(\mathrm{LOEL})=10 \mathrm{ppm}]$. In female rats, a LOEL of $10 \mathrm{ppm}$ was based on splenic congestion, observed in all treated groups (except $30 \mathrm{ppm}$ ); with hematopoietic cell 
proliferation and pigmentation in the spleen, bone marrow hyperplasia, and hematological changes at higher exposure concentrations. This pattern of erythropoietic responses in the spleen and bone marrow and hematologic changes was also observed in male rats at $300 \mathrm{ppm}$ or greater. In male mice, absolute and relative liver weights were significantly higher than controls in all exposed groups (LOEL $10 \mathrm{ppm}$ ), with histologic changes (centrilobular hepatocyte hypertrophy) occurring at 300 and 1,000 ppm. In female mice, absolute and relative liver weights were significantly greater than controls, with increased incidences of centrilobular hepatocyte hypertrophy occurring at similar exposure concentrations (LOEL 300 ppm). 


\section{References}

1. Hawley's condensed chemical dictionary, 13th ed. Lewis R, editor. New York, NY: Van Nostrand Reinhold; 1997.

2. Lide D. CRC handbook of chemistry and physics, 76th ed. Boca Raton, FL: CRC Press. 1995.

3. Reilly 5th edition product index. Indianapolis, IN: Reilly Industries, Inc; 1990.

4. Gehring P. Pyridine, homologues and derivatives In: Parneggiani L, editor. Encyclopedia of Occupational Health and Safety, 3rd revised ed. Geneva, Switzerland: International Labor Office; 1983. p. 1810-1812.

5. Goe G. Pyridine and pyridine derivatives. In: Kirk-Othmer Encyclopedia of Chemical Technology, 3rd ed. New York, NY: John Wiley and Sons; 1982. p. 470.

6. Toomey J, inventor; Reilly Industries Inc, assignee. Pyridine chlorination process. United States patent no. WO 9413640 A1; 1994

7. Sharvit J, Lubetzky D, Pereferkovichm A, inventors; Makhteshim Chemical Works Limited, assignee. Process and catalysts for manufacture of chlorinated pyridines from alpha-picoline. Israel patent no. EP 239905 A1; 1987

8. Tamura M, Kasuga J, Watanabe N, inventors; Mitsubishi Kagaku Kk, assignee. Preparation of chloropyridines. Japan; 1995

9. United States International Trade Commission (USITC). Synthetic organic chemicals, U.S. production and sales. Washington, DC: Government Printing Office (GPO); 1994. USITC Publication No. 2810.

10. United States Environmental Protection Agency (USEPA). Toxic Substance Control Act chemical substances inventory. 2015. https://www.epa.gov/tsca-inventory [Accessed: July 29, 2015]

11. United States Environmental Protection Agency (USEPA). Initial risk-based prioritization of high production volume (HPV) chemicals. Washington, DC: United States Environmental Protection Agency (USEPA); 2009. http://www.epa.gov/hpvis/rbp/109-09-1_2-

Chloropyridine_Web_April\%202009.pdf

12. Kuney J. Chemcyclopedia 95 - The manual of commercially available chemicals.

Washington, DC: The American Chemical Society. 1994; p. 194, 410.

13. Anonymous. Olin doubles biocide precursor. Chem Mark Rep. 1996; 250:4.

14. Aldrich Catalog/Handbook of Fine Chemicals 1996-1997. Milwaukee, WI: Aldrich Chemical Co., Inc; 1996. p. 365.

15. Eastman Laboratory chemicals, catalog no. 55, 93-94 edition. Rochester, NY: Eastman Chemical Company; 1993. 
16. Acros organics 1995-1996 handbook of fine chemicals. Pittsburgh, PA: Fisher Scientific; 1995.

17. United States Environmental Protection Agency (USEPA). Industrial hygiene survey of OlinRochester with cover letter. Washington, DC: United States Environmental Protection Agency (USEPA); 1983. EPA/OTS: Doc \#878220191.

18. National Institute for Occupational Safety and Health (NIOSH). National Occupational Exposure Survey (1981-1983) [unpublished provisional data]. Cincinnati, OH. 1990.

19. Gehring P, Torkelson T, Oyen F. A comparison of the lethality of chlorinated pyridines and a study of the acute toxicity of 2-chloropyridine. Toxicol Appl Pharmacol. 1967; 11(2):361-371. http://dx.doi.org/10.1016/0041-008X(67)90079-8

20. Melcher R, Bouyoucos S. Membrane interface for automatic extraction and liquid chromatographic determination of trace organics in aqueous streams. Process Control Qual. 1990; 1:63-74.

21. Guardiola A, Ventura F, Matia L, Caixach J, Rivera J. Gas chromatographic - mass spectrometric characterization of volatile organic compounds in Barcelona tap water. J Chromatogr. 1991; 562(1-2):481-492. http://dx.doi.org/10.1016/0378-4347(91)80601-8

22. Hendriks A, Maas-Diepeveen J, Noordsij A, Van der Gaag M. Monitoring response of XADconcentrated water in the Rhine delta: A major part of the toxic compounds remains unidentified. Water Res. 1994; 28(3):581-598. http://dx.doi.org/10.1016/0043-1354(94)90009-4

23. Liu SM. Anaerobic dechlorination of chlorinated pyridines in anoxic freshwater sediment slurries. J Environ Sci Health Part A. 1995; 30(3):485-503.

http://dx.doi.org/10.1080/10934529509376213

24. Adrian NR, Suflita JM. Anaerobic biodegradation of halogenated and nonhalogenated N-, S-, and O-heterocyclic compounds in aquifer slurries. Environ Toxicol Chem. 1994; 13(10):15511557.

25. American Conference of Governmental Industrial Hygienists (ACGIH). 2015 TLVs® and BEIs ${ }^{\circledR}$ based on the documentation of the threshold limit values for chemical substances and physical agents \& biological exposure indices. Cincinnati, OH; 2015.

26. The book of chemical lists, vol. 1. Madison, CT: Business and Legal Reports, Inc.; 1995.

27. Chlopkiewicz B, Wojtowicz M, Marczewska J, Prokopczyk D, Koziorowska J. Contribution of N-oxidation and $\mathrm{OH}$ radicals to mutagenesis of 2-chloropyridine in Salmonella typhimurium. Acta Biochim Pol. 1993; 40(1):57-59.

28. Claxton L, Dearfield K, Spanggord R, Riccio E, Mortelmans K. Comparative mutagenicity of halogenated pyridines in the Salmonella typhimurium/mammalian microsome test. Mutat Res. 1987; 176(2):185-198. http://dx.doi.org/10.1016/0027-5107(87)90049-2

29. Zimmermann F, Henning J, Scheel I, Oehler M. Genetic and anti-tubulin effects induced by pyridine derivatives. Mutat Res. 1986; 163(1):23-31. http://dx.doi.org/10.1016/0027-

$\underline{5107(86) 90054-0}$ 
30. Anuszewska E, Koziorowska J. Role of pyridine N-oxide in the cytotoxicity and genotoxicity of chloropyridines. Toxicol In Vitro. 1995; 9(2):91-94. http://dx.doi.org/10.1016/0887$\underline{2333(94) 00199-5}$

31. Dearfield KL, Harrington-Brock K, Doerr CL, Parker L, Moore MM. Genotoxicity of three pyridine compounds to L5178Y mouse lymphoma cells. Mutat Res. 1993; 301(1):57-63. http://dx.doi.org/10.1016/0165-7992(93)90057-3

32. Simmon V, Kauhanen K, Tardiff R, Mortelmans K. Mutagenic activity of chemicals identified in drinking water. Mutat Res. 1978; 53(2):262. https://doi.org/10.1016/0165$\underline{1161(78) 90337-0}$

33. National Toxicology Program (NTP). Toxicology and carcinogenesis studies of pyridine (CAS No. 110-86-1) in F344/N rats, Wistar rats, and B6C3F1 mice (drinking water studies). Research Triangle Park, NC: U.S. Department of Health and Human Services, Public Health Service, National Institutes of Health; 2000. Technical Report Series No. 470. NIH Publication No. 00-3960.

34. Maronpot R, Boorman G. Interpretation of rodent hepatocellular proliferative alterations and hepatocellular tumors in chemical safety assessment. Toxicol Pathol. 1982; 10(2):71-78. http://dx.doi.org/10.1177/019262338201000210

35. Boorman GA, Montgomery CA, Jr., Eustis SL, Wolfe MJ, McConnell EE, Hardisty JF. Quality assurance in pathology for rodent carcinogenicity studies. In: Milman HA, Weisburger EK, editors. Handbook of Carcinogen Testing. Park Ridge, NJ: Noyes Publications; 1985. p. 345-357.

36. Gart JJ, Chu KC, Tarone RE. Statistical issues in interpretation of chronic bioassay tests for carcinogenicity. J Natl Cancer Inst. 1979; 62(4):957-974.

37. Dunnett CW. A multiple comparison procedure for comparing several treatments with a control. J Am Stat Assoc. 1955; 50(272):1096-1121.

http://dx.doi.org/10.1080/01621459.1955.10501294

38. Williams D. The comparison of several dose levels with a zero dose control. Biometrics. 1972; 28(2):519-531. http://dx.doi.org/10.2307/2556164

39. Williams D. A test for differences between treatment means when several dose levels are compared with a zero dose control. Biometrics. 1971; 27(1):103-117.

http://dx.doi.org/10.2307/2528930

40. Shirley E. A non-parametric equivalent of Williams' test for contrasting increasing dose levels of a treatment. Biometrics. 1977; 33(2):386-389. http://dx.doi.org/10.2307/2529789

41. Williams D. A note on Shirley's nonparametric test for comparing several dose levels with a zero-dose control. Biometrics. 1986; 42(1):183-186. http://dx.doi.org/10.2307/2531254

42. Dunn OJ. Multiple comparisons using rank sums. Technometrics. 1964; 6(3):241-252.

http://dx.doi.org/10.1080/00401706.1964.10490181 
43. Jonckheere A. A distribution-free k-sample test against ordered alternatives. Biometrika. $1954 ; 41: 133-145$.

44. Dixon W, Massey F. Introduction to statistical analysis. New York, NY: McGraw Hill Book Company Inc; 1957. http://dx.doi.org/10.2307/2332898

45. Girard D, Sager D. The use of Markov chains to detect subtle variation in reproductive cycling. Biometrics. 1987; 43(1):225-234. http://dx.doi.org/10.2307/2531963

46. Code of Federal Regulations (CFR). 21:Part 58.

47. Zeiger E, Anderson B, Haworth S, Lawlor T, Mortelmans K. Salmonella mutagenicity tests. V. Results from the testing of 311 chemicals. Environ Mol Mutagen. 1992; 19(S21):2-141. http://dx.doi.org/10.1002/em.2850190603

48. MacGregor JT, Wehr CM, Henika PR, Shelby MD. The in vivo erythrocyte micronucleus test: Measurement at steady state increases assay efficiency and permits integration with toxicity studies. Fundam Appl Toxicol. 1990; 14(3):513-522. http://dx.doi.org/10.1016/0272$\underline{0590(90) 90255-\mathrm{I}}$

49. Stockham S, Scott M. Erythrocytes. Fundamentals of veterinary clinical pathology, 2nd ed. Ames, IA: Blackwell Publishing; 2008. p. 107-221.

50. Klauder DS, Petering HG. Anemia of lead intoxication: A role for copper. J Nutr. 1977; 107(10):1779-1785. http://dx.doi.org/10.1093/jn/107.10.1779

51. National Toxicology Program (NTP). Toxicity studies of cupric sulfate (CAS No. 7758-99-8) administered in drinking water and feed to F344/N rats and B6C3F1 mice. Research Triangle Park, NC: U.S. Department of Health and Human Services, Public Health Service, National Institutes of Health; 1993. Toxicity Report Series No. 29. NIH Publication No. 93-3352.

52. National Toxicology Program (NTP). Toxicity studies of estragole (CAS No. 140-67-0) administered by gavage to F344/N rats and B6C3F1 mice. Research Triangle Park, NC: U.S. Department of Health and Human Services, Public Health Service, National Institutes of Health; 2011. Toxicity Report Series No. 82. NIH Publication No. 11-5966.

53. Matuoka K, Markus I, Wong A, Smith GJ. Diethylnitrosamine-and partial hepatectomyinduced decrease in a 2u-globulin mRNA level in the rat liver. J Cancer Res Clin Oncol. 1993; 119(10):572-575. http://dx.doi.org/10.1007/BF01372719

54. National Toxicology Program (NTP). Toxicology and carcinogenesis studies of $\beta$-picoline (CAS No. 108 99-6) in F344/N rats and B6C3F1/N mice (drinking water studies). U.S. Department of Health and Human Services, Public Health Service, National Institutes of Health: Research Triangle Park, NC; 2014. Technical Report Series No. 580. NIH Publication No. 145922.

55. The Aldrich Library of $13 \mathrm{C}$ and $1 \mathrm{H}$ FT-NMR Spectra. 1st ed. Vol. 3. Milwaukee, WI: Aldrich Chemical Co., Inc.; 1992. p. 260C.

56. The Aldrich Library of FT-IR Spectra. 1st ed. Vol. 2, Spectrum 2. Milwaukee, WI: SigmaAldrich Chemical Company; 1985. p. 746A. 
57. The Aldrich library of infrared spectra. 3rd ed. Vol. 3, Spectrum 66B. Pouchert CJ, editor. Milwaukee, WI: Aldrich Chemical Company Inc.; 1981.

58. Talik Z. Chem. Abstr. Roczniki Chemii. 1962; 36(59):1313-1320.

59. Beak P, Lee Jr JT. Equilibration studies. 2-(Methylthio) pyridine 1-methyl-2 (1H)pyridinethione. J Org Chem. 1969; 34(7):2125-2128. http://dx.doi.org/10.1021/jo01259a021

60. Westland RD, Cooley Jr RA, Holmes JL, Hong JS, Lin MH, Zwiesler ML. Antiradiation agents. Substituted 2-pyridyloxy and 2-quinolyloxy derivatives of S-2-(alkylamino) ethyl hydrogen thiosulfates and 3-alkylthiazolidines and substituted 2-pyridyloxy derivatives of 2(alkylamino) ethanethiols and corresponding disulfides. J Med Chem. 1973; 16(4):319-327. http://dx.doi.org/10.1021/jm00262a003

61. Kohrman R, West D, Little M. The thermal deoxygenation of 2-alkylthiopyridine 1-oxides. J Heterocycl Chem. 1974; 11(1):101-102. http://dx.doi.org/10.1002/jhet.5570110126

62. McKenna M, Bieri J. Multilayer cannula for long-term infusion of unrestrained rats. Lab Anim Sci. 1984; 34(3):308-310.

63. Gorrod J, Damani L. The metabolic N-oxidation of 3-substituted pyridines in various animal species in vivo. Eur J Drug Metab Pharmacokinet. 1980; 5(1):53-57.

http://dx.doi.org/10.1007/BF03189445

64. Damani L, Crooks P, Shaker M, Caldwell J, D'souza J, Smith R. Species differences in the metabolic C-and N-oxidation, and N-methylation of [14C] pyridine in vivo. Xenobiotica. 1982; 12(8):527-534. http://dx.doi.org/10.3109/00498258209038931

65. Shaker M, Crooks P, Damani L. High-performance liquid chromatographic analysis of the in vivo metabolites of [14C] pyridine. J Chromatogr A. 1982; 237(3):489-495.

http://dx.doi.org/10.1016/S0021-9673(00)97638-6

66. D'souza J, Caldwell J, Smith R. Species variations in the N-methylation and quaternization of [14C] pyridine. Xenobiotica. 1980; 10(2):151-157.

http://dx.doi.org/10.3109/00498258009033741

67. Donaldson HH, Conrow S. Quantitative studies on the growth of the skeleton of the albino rat. Am J Anat. 1919; 26(2):236-314. http://dx.doi.org/10.1002/aja.1000260204

68. Adolph EF. Quantitative relations in the physiological constitutions of mammals. Science. 1949; 109(2841):579-585. http://dx.doi.org/10.1126/science.109.2841.579

69. Supplee H, Hauschildt JD, Entenman C. Plasma proteins and plasma volume in rats following total-body X-irradiation. Am J Physiol. 1952; 169(2):483-490. http://dx.doi.org/10.1152/ajplegacy.1952.169.2.483

70. Caster W, Poncelet J, Simon AB, Armstrong W. Tissue weights of the rat. I. Normal values determined by dissection and chemical methods. Proc Soc Exp Biol Med. 1956; 91(1):122-126. http://dx.doi.org/10.3181/00379727-91-22186 
71. Bischoff K, Dedrick R, Zaharko D, Longstreth J. Methotrexate pharmacokinetics. J Pharm Sci. 1971; 60(8):1128-1133. http://dx.doi.org/10.1002/jps.2600600803

72. Lutz R, Dedrick R, Matthews H, Eling T, Anderson M. A preliminary pharmacokinetic model for several chlorinated biphenyls in the rat. Drug Metab Disposition. 1977; 5(4):386-396. 


\section{Appendix A. Summary of Lesions in Rats and Mice}

\section{Tables}

Table A-1. Summary of the Incidence of Nonneoplastic Lesions in Male Rats in the

Three-month Drinking Water Study of $o$-Chloropyridine.

Table A-2. Summary of the Incidence of Nonneoplastic Lesions in Female Rats in the Three-month Drinking Water Study of $o$-Chloropyridine.

Table A-3. Summary of the Incidence of Neoplasms and Nonneoplastic Lesions in Male Mice in the Three-month Drinking Water Study of $o$-Chloropyridine

Table A-4. Summary of the Incidence of Neoplasms and Nonneoplastic Lesions in

Female Mice in the Three-month Drinking Water Study of $o$-Chloropyridine A-8 
Table A-1. Summary of the Incidence of Nonneoplastic Lesions in Male Rats in the Three-month Drinking Water Study of $o$-Chloropyridine ${ }^{a}$

\begin{tabular}{|c|c|c|c|c|c|c|}
\hline & O ppm & $10 \mathrm{ppm}$ & 30 ppm & 100 ppm & 300 ppm & $1,000 \mathrm{ppm}$ \\
\hline \multicolumn{7}{|l|}{ Disposition Summary } \\
\hline Animals initially in study & 10 & 10 & 10 & 10 & 10 & 10 \\
\hline \multicolumn{7}{|l|}{ Survivors } \\
\hline Terminal sacrifice & 10 & 10 & 10 & 10 & 10 & 10 \\
\hline $\begin{array}{l}\text { Animals examined } \\
\text { microscopically }\end{array}$ & 10 & 10 & 10 & 10 & 10 & 10 \\
\hline \multicolumn{7}{|l|}{ Alimentary System } \\
\hline Liver & (10) & (10) & (10) & $(10)$ & (10) & (10) \\
\hline Basophilic focus & 0 & 0 & 0 & 0 & 0 & $2(20 \%)$ \\
\hline Clear cell focus & 0 & 0 & 0 & 0 & 0 & $6(60 \%)$ \\
\hline Eosinophilic focus & 0 & 0 & 0 & 0 & 0 & $1(10 \%)$ \\
\hline $\begin{array}{l}\text { Hepatodiaphragmatic } \\
\text { nodule }\end{array}$ & 0 & 0 & 0 & 0 & $1(10 \%)$ & 0 \\
\hline Bile duct, hyperplasia & $1(10 \%)$ & 0 & 0 & 0 & 0 & 0 \\
\hline $\begin{array}{l}\text { Hepatocyte, } \\
\text { vacuolization cytoplasmic }\end{array}$ & 0 & 0 & 0 & 0 & $6(60 \%)$ & $9(90 \%)$ \\
\hline Salivary glands & (9) & (0) & (0) & (0) & (0) & (10) \\
\hline $\begin{array}{l}\text { Parotid gland, } \\
\text { basophilic focus }\end{array}$ & $2(22 \%)$ & - & - & - & - & $5(50 \%)$ \\
\hline \multicolumn{7}{|l|}{ Cardiovascular System } \\
\hline Heart & $(10)$ & (0) & (0) & (0) & (0) & (10) \\
\hline Cardiomyopathy & $4(40 \%)$ & - & - & - & - & $2(20 \%)$ \\
\hline Inflammation, chronic & $1(10 \%)$ & - & - & - & - & 0 \\
\hline \multicolumn{7}{|l|}{ Endocrine System } \\
\hline Adrenal cortex & (10) & (0) & (0) & (0) & (0) & (10) \\
\hline $\begin{array}{l}\text { Zona fasciculata, } \\
\text { vacuolization cytoplasmic }\end{array}$ & $9(90 \%)$ & - & - & - & - & $5(50 \%)$ \\
\hline $\begin{array}{l}\text { Zona reticularis, } \\
\text { vacuolization cytoplasmic }\end{array}$ & 0 & - & - & - & - & $2(20 \%)$ \\
\hline Thyroid gland & (10) & (0) & (0) & (0) & (0) & (10) \\
\hline Cyst & $1(10 \%)$ & - & - & - & - & 0 \\
\hline Ectopic thymus & $1(10 \%)$ & - & - & - & - & $1(10 \%)$ \\
\hline
\end{tabular}

\section{General Body System}

None

\section{Genital System}

None

\section{Hematopoietic System}

Bone marrow

(10)

(10)

(10)

(10)

(10)

(10)

Hyperplasia

$1(10 \%)$

$0 \quad 2(20 \%)$

$1(10 \%)$

$9(90 \%)$

$6(60 \%)$ 
$o$-Chloropyridine, NTP TOX 83

\begin{tabular}{lcccccc}
\hline & $\mathbf{0 ~ p p m}$ & $\mathbf{1 0} \mathbf{~ p p m}$ & $\mathbf{3 0} \mathbf{~ p p m}$ & $\mathbf{1 0 0} \mathbf{p p m}$ & $\mathbf{3 0 0} \mathbf{p p m}$ & $\mathbf{1 , 0 0 0} \mathbf{~ p p m}$ \\
\hline Lymph node, mesenteric & $(10)$ & $(0)$ & $(0)$ & $(0)$ & $(0)$ & $(10)$ \\
$\quad$ Sinus, histiocytosis & 0 & - & - & - & - & $2(20 \%)$ \\
Spleen & $(10)$ & $(10)$ & $(10)$ & $(10)$ & $(10)$ & $(10)$ \\
$\quad$ Congestion & $4(40 \%)$ & $1(10 \%)$ & $2(20 \%)$ & 0 & $7(70 \%)$ & $10(100 \%)$ \\
Hematopoietic cell & $4(40 \%)$ & $8(80 \%)$ & $6(60 \%)$ & $6(60 \%)$ & $10(100 \%)$ & $10(100 \%)$ \\
$\quad$ proliferation & & & & & & \\
$\quad$ Pigmentation & $10(100 \%)$ & $10(100 \%)$ & $10(100 \%)$ & $10(100 \%)$ & $10(100 \%)$ & $10(100 \%)$ \\
\hline
\end{tabular}

\section{Integumentary System}

None

\section{Musculoskeletal System}

None

\section{Nervous System}

None

\section{Respiratory System}

\begin{tabular}{|c|c|c|c|c|c|c|}
\hline Lung & (10) & (0) & (0) & $(0)$ & (0) & (10) \\
\hline $\begin{array}{l}\text { Infiltration cellular, } \\
\text { mononuclear cell, focal }\end{array}$ & $1(10 \%)$ & - & - & - & - & 0 \\
\hline Metaplasia, osseous & $2(20 \%)$ & - & - & - & - & 0 \\
\hline Alveolus, hemorrhage, focal & $1(10 \%)$ & - & - & - & - & $1(10 \%)$ \\
\hline \multicolumn{7}{|l|}{ Special Senses System } \\
\hline Eye & (10) & (0) & (0) & $(0)$ & $(0)$ & $(10)$ \\
\hline $\begin{array}{l}\text { Posterior chamber, } \\
\text { developmental } \\
\text { malformation }\end{array}$ & $1(10 \%)$ & - & - & - & - & 0 \\
\hline \multicolumn{7}{|l|}{ Urinary System } \\
\hline Kidney & (10) & $(10)$ & $(10)$ & $(10)$ & (10) & (10) \\
\hline Mineralization & 0 & $1(10 \%)$ & 0 & 0 & 0 & 0 \\
\hline Nephropathy & $3(30 \%)$ & $2(20 \%)$ & $5(50 \%)$ & $6(60 \%)$ & $3(30 \%)$ & $4(40 \%)$ \\
\hline $\begin{array}{l}\text { Renal tubule, accumulation, } \\
\text { hyaline droplet }\end{array}$ & $10(100 \%)$ & $10(100 \%)$ & $10(100 \%)$ & $9(90 \%)$ & $5(50 \%)$ & 0 \\
\hline
\end{tabular}

${ }^{a}$ Number of animals examined microscopically at the site and the number of animals with lesion. 
Table A-2. Summary of the Incidence of Nonneoplastic Lesions in Female Rats in the Three-month Drinking Water Study of $o$-Chloropyridine ${ }^{\mathrm{a}}$

\begin{tabular}{|c|c|c|c|c|c|c|}
\hline & O ppm & 10 ppm & 30 ppm & 100 ppm & 300 ppm & 1,000 ppm \\
\hline \multicolumn{7}{|l|}{ Disposition Summary } \\
\hline Animals initially in study & 10 & 10 & 10 & 10 & 10 & 10 \\
\hline \multicolumn{7}{|l|}{ Survivors } \\
\hline Terminal sacrifice & 10 & 10 & 10 & 10 & 10 & 10 \\
\hline $\begin{array}{l}\text { Animals examined } \\
\text { microscopically }\end{array}$ & 10 & 10 & 10 & 10 & 10 & 10 \\
\hline \multicolumn{7}{|l|}{ Alimentary System } \\
\hline Liver & (10) & (10) & (10) & $(10)$ & (10) & $(10)$ \\
\hline Clear cell focus & 0 & 0 & 0 & 0 & 0 & $2(20 \%)$ \\
\hline $\begin{array}{l}\text { Hepatocyte, vacuolization } \\
\text { cytoplasmic }\end{array}$ & 0 & 0 & 0 & 0 & 0 & $9(90 \%)$ \\
\hline Pancreas & (10) & $(0)$ & (0) & (0) & (0) & $(10)$ \\
\hline Duct, hyperplasia & $1(10 \%)$ & - & - & - & - & 0 \\
\hline Salivary glands & (10) & (0) & (0) & (0) & (0) & (10) \\
\hline $\begin{array}{l}\text { Parotid gland, } \\
\text { basophilic focus }\end{array}$ & $3(30 \%)$ & - & - & - & - & $2(20 \%)$ \\
\hline \multicolumn{7}{|l|}{ Cardiovascular System } \\
\hline Heart & (10) & (0) & (0) & (0) & (0) & (10) \\
\hline Cardiomyopathy & $1(10 \%)$ & - & - & - & - & 0 \\
\hline Epicardium, inflammation & 0 & - & - & - & - & $1(10 \%)$ \\
\hline \multicolumn{7}{|l|}{ Endocrine System } \\
\hline Adrenal cortex & (10) & (0) & (0) & (0) & (0) & $(10)$ \\
\hline $\begin{array}{l}\text { Infiltration cellular, } \\
\text { mononuclear cell, focal }\end{array}$ & 0 & - & - & - & - & $1(10 \%)$ \\
\hline Pituitary gland & (9) & (0) & (0) & (0) & (0) & $(10)$ \\
\hline Pars distalis, cyst & $1(11 \%)$ & - & - & - & - & 0 \\
\hline Thyroid gland & $(10)$ & (0) & (0) & (0) & (0) & $(10)$ \\
\hline Cyst & $2(20 \%)$ & - & - & - & - & $1(10 \%)$ \\
\hline Ectopic thymus & $1(10 \%)$ & - & - & - & - & 0 \\
\hline \multicolumn{7}{|l|}{ General Body System } \\
\hline None & - & - & - & - & - & - \\
\hline \multicolumn{7}{|l|}{ Genital System } \\
\hline Uterus & $(10)$ & $(0)$ & (0) & (0) & (0) & $(10)$ \\
\hline Dilatation & $1(10 \%)$ & - & - & - & - & $3(30 \%)$ \\
\hline Hydrometra & $1(10 \%)$ & - & - & - & - & $2(20 \%)$ \\
\hline \multicolumn{7}{|l|}{ Hematopoietic System } \\
\hline Bone marrow & (10) & $(10)$ & $(10)$ & (10) & (10) & $(10)$ \\
\hline Hyperplasia & 0 & 0 & 0 & 0 & $3(30 \%)$ & $9(90 \%)$ \\
\hline
\end{tabular}


$o$-Chloropyridine, NTP TOX 83

\begin{tabular}{|c|c|c|c|c|c|c|}
\hline & 0 ppm & 10 ppm & 30 ppm & 100 ppm & 300 ppm & 1,000 ppm \\
\hline Lymph node, mesenteric & $(10)$ & $(0)$ & $(0)$ & $(0)$ & (0) & $(10)$ \\
\hline Sinus, histiocytosis & $6(60 \%)$ & - & - & - & - & $4(40 \%)$ \\
\hline Spleen & $(10)$ & $(10)$ & (10) & $(10)$ & (10) & $(10)$ \\
\hline Congestion & $1(10 \%)$ & $7(70 \%)$ & $5(50 \%)$ & $6(60 \%)$ & $6(60 \%)$ & $10(100 \%)$ \\
\hline $\begin{array}{l}\text { Hematopoietic } \\
\text { cell proliferation }\end{array}$ & $3(30 \%)$ & $3(30 \%)$ & $6(60 \%)$ & $3(30 \%)$ & $7(70 \%)$ & $10(100 \%)$ \\
\hline Pigmentation & $10(100 \%)$ & $10(100 \%)$ & $10(100 \%)$ & $10(100 \%)$ & $10(100 \%)$ & $10(100 \%)$ \\
\hline Thymus & $(10)$ & $(0)$ & $(0)$ & $(0)$ & $(0)$ & $(10)$ \\
\hline Thymocyte, atrophy & $1(10 \%)$ & - & - & - & - & 0 \\
\hline \multicolumn{7}{|l|}{ Integumentary System } \\
\hline None & - & - & - & - & - & - \\
\hline \multicolumn{7}{|l|}{ Musculoskeletal System } \\
\hline None & - & - & - & - & - & - \\
\hline \multicolumn{7}{|l|}{ Nervous System } \\
\hline None & - & - & - & - & - & - \\
\hline \multicolumn{7}{|l|}{ Respiratory System } \\
\hline Lung & $(10)$ & $(0)$ & $(0)$ & $(0)$ & $(0)$ & $(10)$ \\
\hline $\begin{array}{l}\text { Infiltration cellular, } \\
\text { mononuclear cell, focal }\end{array}$ & $4(40 \%)$ & - & - & - & - & $3(30 \%)$ \\
\hline $\begin{array}{l}\text { Alveolus, hemorrhage, } \\
\text { focal }\end{array}$ & $1(10 \%)$ & - & - & - & - & 0 \\
\hline \multicolumn{7}{|l|}{ Special Senses System } \\
\hline Eye & $(10)$ & $(0)$ & $(0)$ & $(0)$ & $(0)$ & $(10)$ \\
\hline Cornea, inflammation & 0 & - & - & - & - & $1(10 \%)$ \\
\hline Sclera, inflammation & 0 & - & - & - & - & $2(20 \%)$ \\
\hline Harderian gland & $(10)$ & (0) & $(0)$ & $(0)$ & $(0)$ & $(10)$ \\
\hline $\begin{array}{l}\text { Infiltration cellular } \\
\text { developmental } \\
\text { malformation }\end{array}$ & 0 & - & - & - & - & $1(10 \%)$ \\
\hline \multicolumn{7}{|l|}{ Urinary System } \\
\hline Kidney & $(10)$ & $(10)$ & $(10)$ & $(10)$ & $(10)$ & $(10)$ \\
\hline Mineralization & 0 & 0 & $1(10 \%)$ & 0 & 0 & 0 \\
\hline Nephropathy & $2(20 \%)$ & 0 & $1(10 \%)$ & $1(10 \%)$ & $2(20 \%)$ & $5(50 \%)$ \\
\hline
\end{tabular}


Table A-3. Summary of the Incidence of Neoplasms and Nonneoplastic Lesions in Male Mice in the Three-month Drinking Water Study of $o$-Chloropyridine ${ }^{a}$

\begin{tabular}{|c|c|c|c|c|c|c|}
\hline & O ppm & $10 \mathrm{ppm}$ & $30 \mathrm{ppm}$ & $100 \mathrm{ppm}$ & 300 ppm & $1,000 \mathrm{ppm}$ \\
\hline \multicolumn{7}{|l|}{ Disposition Summary } \\
\hline Animals initially in study & 10 & 10 & 10 & 10 & 10 & 10 \\
\hline \multicolumn{7}{|l|}{ Survivors } \\
\hline Terminal sacrifice & 10 & 10 & 10 & 10 & 10 & 10 \\
\hline $\begin{array}{c}\text { Animals examined } \\
\text { microscopically }\end{array}$ & 10 & 10 & 10 & 10 & 10 & 10 \\
\hline \multicolumn{7}{|l|}{ Alimentary System } \\
\hline Liver & (10) & $(10)$ & (10) & $(10)$ & $(10)$ & (10) \\
\hline $\begin{array}{l}\text { Infiltration cellular, } \\
\text { mononuclear cell, focal }\end{array}$ & $1(10 \%)$ & 0 & $1(10 \%)$ & 0 & $1(10 \%)$ & $1(10 \%)$ \\
\hline Necrosis, focal & $1(10 \%)$ & 0 & $2(20 \%)$ & 0 & 0 & $2(20 \%)$ \\
\hline $\begin{array}{l}\text { Centrilobular, hepatocyte, } \\
\text { hypertrophy }\end{array}$ & 0 & 0 & 0 & $1(10 \%)$ & $6(60 \%)$ & $9(90 \%)$ \\
\hline Stomach, glandular & (10) & (0) & (0) & (0) & (0) & (10) \\
\hline Cyst & 0 & - & - & - & - & $1(10 \%)$ \\
\hline Cyst epithelial inclusion & $2(20 \%)$ & - & - & - & - & 0 \\
\hline \multicolumn{7}{|l|}{ Cardiovascular System } \\
\hline None & - & - & - & - & - & - \\
\hline \multicolumn{7}{|l|}{ Endocrine System } \\
\hline Adrenal cortex & (9) & (0) & (0) & (0) & (0) & (10) \\
\hline $\begin{array}{l}\text { Subcapsular, hyperplasia } \\
\text { focal }\end{array}$ & 0 & - & - & - & - & $2(20 \%)$ \\
\hline Parathyroid gland & (7) & (0) & (0) & (0) & (0) & (7) \\
\hline Cyst & $1(14 \%)$ & - & - & - & - & 0 \\
\hline \multicolumn{7}{|l|}{ General Body System } \\
\hline None & - & - & - & - & - & - \\
\hline \multicolumn{7}{|l|}{ Genital System } \\
\hline None & - & - & - & - & - & - \\
\hline \multicolumn{7}{|l|}{ Hematopoietic System } \\
\hline Spleen & (10) & (0) & (0) & (0) & (0) & (10) \\
\hline $\begin{array}{l}\text { Hematopoietic cell } \\
\text { proliferation }\end{array}$ & $10(100 \%)$ & - & - & - & - & $10(100 \%)$ \\
\hline \multicolumn{7}{|l|}{ Integumentary System } \\
\hline None & - & - & - & - & - & - \\
\hline \multicolumn{7}{|l|}{ Musculoskeletal System } \\
\hline None & - & - & - & - & - & - \\
\hline
\end{tabular}


$o$-Chloropyridine, NTP TOX 83

\begin{tabular}{|c|c|c|c|c|c|c|}
\hline & O ppm & $10 \mathrm{ppm}$ & 30 ppm & 100 ppm & 300 ppm & 1,000 ppm \\
\hline \multicolumn{7}{|l|}{ Nervous System } \\
\hline None & - & - & - & - & - & - \\
\hline \multicolumn{7}{|l|}{ Respiratory System } \\
\hline None & - & - & - & - & - & - \\
\hline \multicolumn{7}{|l|}{ Special Senses System } \\
\hline None & - & - & - & - & - & - \\
\hline \multicolumn{7}{|l|}{ Urinary System } \\
\hline Kidney & (10) & (10) & $(10)$ & (10) & (10) & (10) \\
\hline Mineralization & 0 & 0 & 0 & 0 & $2(20 \%)$ & 0 \\
\hline Nephropathy & 0 & 0 & 0 & 0 & 0 & $1(10 \%)$ \\
\hline $\begin{array}{l}\text { Interstitium, } \\
\text { infiltration cellular, } \\
\text { mononuclear cell }\end{array}$ & 0 & 0 & $1(10 \%)$ & 0 & 0 & $1(10 \%)$ \\
\hline Renal tubule, adenoma & 0 & 0 & $1(10 \%)$ & 0 & 0 & 0 \\
\hline Renal tubule, casts protein & 0 & 0 & 0 & $1(10 \%)$ & 0 & 0 \\
\hline $\begin{array}{l}\text { Renal tubule, } \\
\text { vacuolization cytoplasmic }\end{array}$ & $10(100 \%)$ & $10(100 \%)$ & $10(100 \%)$ & $10(100 \%)$ & $10(100 \%)$ & $10(100 \%)$ \\
\hline
\end{tabular}

${ }^{a}$ Number of animals examined microscopically at the site and the number of animals with lesion. 
Table A-4. Summary of the Incidence of Neoplasms and Nonneoplastic Lesions in Female Mice in the Three-month Drinking Water Study of $o$-Chloropyridine ${ }^{a}$

\begin{tabular}{lcccccc}
\hline & $\mathbf{0 ~ p p m}$ & $\mathbf{1 0} \mathbf{~ p p m}$ & $\mathbf{3 0} \mathbf{~ p p m}$ & $\mathbf{1 0 0} \mathbf{~ p p m}$ & $\mathbf{3 0 0} \mathbf{~ p p m}$ & $\mathbf{1 , 0 0 0} \mathbf{~ p p m}$ \\
\hline $\begin{array}{l}\text { Disposition Summary } \\
\begin{array}{l}\text { Animals initially in study } \\
\text { Early deaths }\end{array}\end{array}$ & 10 & 10 & 10 & 10 & 10 & 10 \\
$\quad$ Natural death & 1 & - & - & - & - & - \\
$\begin{array}{l}\text { Survivors } \\
\quad \text { Terminal sacrifice }\end{array}$ & 9 & 10 & 10 & 10 & 10 & 10 \\
$\begin{array}{l}\text { Animals examined } \\
\text { microscopically }\end{array}$ & 10 & 10 & 10 & 10 & 10 & 10 \\
\hline $\begin{array}{l}\text { Alimentary System } \\
\text { Liver }\end{array}$ & & & & & & \\
$\quad \begin{array}{l}\text { Infiltration cellular, } \\
\text { mononuclear cell, focal }\end{array}$ & 0 & $1(10 \%)$ & $1(10 \%)$ & 0 & $1(10 \%)$ & 0 \\
$\quad \begin{array}{l}\text { Necrosis, focal } \\
\quad \text { Centrilobular, hepatocyte, } \\
\text { hypertrophy }\end{array}$ & 0 & 0 & 0 & 0 & 0 & $1(10 \%)$ \\
\hline
\end{tabular}

\section{Cardiovascular System}

None

\section{Endocrine System}

Adrenal cortex

(10)

(0)

(0)

(0)

(0)

(10)

Subcapsular, hyperplasia, $\quad 10(100 \%)$

$-$

$-$

$10(100 \%)$ focal

\section{General Body System}

None

\section{Genital System}

Ovary

(0)

(0)

(0)

(0)

(10)

Teratoma, benign

$1(10 \%)$

Uterus

(0)

(0)

$-$

Deciduoma, benign

0

Endometrium, hyperplasia

$4(40 \%)$

$-$

(0)

(0) cystic

\section{Hematopoietic System}

Spleen

(9)

(0)

(0)

(0)

(0)

Hematopoietic

$9(100 \%)$ cell proliferation

\section{Integumentary System}

Skin

(10)

(0)

(0)

(0)

(0) 
$o$-Chloropyridine, NTP TOX 83

\begin{tabular}{|c|c|c|c|c|c|c|}
\hline & 0 ppm & 10 ppm & 30 ppm & 100 ppm & 300 ppm & 1,000 ppm \\
\hline Dermis, inflammation & $1(10 \%)$ & - & - & - & - & 0 \\
\hline \multicolumn{7}{|l|}{ Musculoskeletal System } \\
\hline None & - & - & - & - & - & - \\
\hline \multicolumn{7}{|l|}{ Nervous System } \\
\hline None & - & - & - & - & - & - \\
\hline Respiratory System & (10) & (0) & (0) & (0) & (0) & (10) \\
\hline \multicolumn{7}{|l|}{ Lung } \\
\hline Hemorrhage, focal & 0 & - & - & - & - & $1(10 \%)$ \\
\hline \multicolumn{7}{|l|}{ Special Senses System } \\
\hline None & - & - & - & - & - & - \\
\hline \multicolumn{7}{|l|}{ Urinary System } \\
\hline Kidney & (10) & (10) & (10) & (10) & (10) & (10) \\
\hline $\begin{array}{l}\text { Infiltration cellular, } \\
\text { mononuclear cell }\end{array}$ & 0 & 0 & $1(10 \%)$ & 0 & 0 & 0 \\
\hline Mineralization & 0 & 0 & $1(10 \%)$ & 0 & 0 & 0 \\
\hline Nephropathy & 0 & $2(20 \%)$ & 0 & 0 & 0 & 0 \\
\hline $\begin{array}{l}\text { Interstitium, } \\
\text { infiltration cellular, } \\
\text { mononuclear cell }\end{array}$ & $1(10 \%)$ & 0 & 0 & 0 & 0 & 0 \\
\hline
\end{tabular}




\section{Appendix B. Clinical Pathology Results}

\section{Tables}

Table B-1. Hematology and Clinical Chemistry Data for Rats in the Three-month Drinking Water Study of $o$-Chloropyridine .

Table B-2. Hematology Data for Mice in the Three-month Drinking Water Study of $o$-Chloropyridine 
$o$-Chloropyridine, NTP TOX 83

Table B-1. Hematology and Clinical Chemistry Data for Rats in the Three-month Drinking Water Study of $o$-Chloropyridine ${ }^{a}$

\begin{tabular}{|c|c|c|c|c|c|c|}
\hline & O ppm & $10 \mathrm{ppm}$ & $30 \mathrm{ppm}$ & 100 ppm & 300 ppm & 1,000 ppm \\
\hline \multicolumn{7}{|l|}{ Male } \\
\hline \multicolumn{7}{|c|}{ Hematology } \\
\hline \multicolumn{7}{|l|}{$\mathbf{n}$} \\
\hline Day 4 & 10 & 10 & 10 & 9 & 9 & 10 \\
\hline Day 22 & 10 & 10 & 10 & 10 & 10 & 10 \\
\hline Week 14 & 9 & 10 & 10 & 10 & 10 & 10 \\
\hline \multicolumn{7}{|c|}{ Hematocrit (\%) } \\
\hline Day 4 & $40.0 \pm 0.4$ & $39.8 \pm 0.4$ & $40.5 \pm 0.6$ & $38.8 \pm 0.5$ & $39.6 \pm 0.5$ & $43.3 \pm 0.9 *$ \\
\hline Day 22 & $43.4 \pm 0.4$ & $42.7 \pm 0.4$ & $42.6 \pm 0.4$ & $41.2 \pm 0.6^{* *}$ & $41.5 \pm 0.5^{* *}$ & $39.6 \pm 0.4^{* *}$ \\
\hline Week 14 & $45.5 \pm 0.6$ & $45.2 \pm 0.3$ & $46.3 \pm 0.3$ & $44.5 \pm 0.9$ & $42.9 \pm 0.4 * *$ & $41.7 \pm 0.4 * *$ \\
\hline \multicolumn{7}{|c|}{ Hemoglobin (g/dL) } \\
\hline Day 4 & $13.4 \pm 0.1$ & $13.4 \pm 0.1$ & $13.6 \pm 0.2$ & $13.1 \pm 0.2$ & $13.3 \pm 0.2$ & $14.5 \pm 0.3 *$ \\
\hline Day 22 & $14.8 \pm 0.1$ & $14.5 \pm 0.1$ & $14.5 \pm 0.1$ & $14.1 \pm 0.2 * *$ & $14.1 \pm 0.2 * *$ & $13.5 \pm 0.1 * *$ \\
\hline Week 14 & $15.2 \pm 0.2$ & $15.1 \pm 0.1$ & $15.4 \pm 0.1$ & $15.0 \pm 0.3$ & $14.4 \pm 0.1^{* *}$ & $13.9 \pm 0.1 * *$ \\
\hline \multicolumn{7}{|c|}{ Erythrocytes $\left(10^{6} / \mu \mathrm{L}\right)$} \\
\hline Day 4 & $6.80 \pm 0.08$ & $6.85 \pm 0.10$ & $6.92 \pm 0.10$ & $6.72 \pm 0.09$ & $6.95 \pm 0.10$ & $7.53 \pm 0.14^{* *}$ \\
\hline Day 22 & $7.50 \pm 0.07$ & $7.35 \pm 0.09$ & $7.37 \pm 0.08$ & $7.18 \pm 0.11^{*}$ & $7.30 \pm 0.08$ & $7.25 \pm 0.08$ \\
\hline Week 14 & $8.91 \pm 0.11$ & $8.75 \pm 0.07$ & $9.04 \pm 0.08$ & $8.76 \pm 0.15$ & $8.51 \pm 0.06^{* *}$ & $8.42 \pm 0.09 * *$ \\
\hline \multicolumn{7}{|c|}{ Reticulocytes $\left(10^{6} / \mu \mathrm{L}\right)$} \\
\hline Day 4 & $0.46 \pm 0.04$ & $0.45 \pm 0.04$ & $0.54 \pm 0.02$ & $0.50 \pm 0.05$ & $0.46 \pm 0.03$ & $0.39 \pm 0.02$ \\
\hline Day 22 & $0.33 \pm 0.04$ & $0.33 \pm 0.02$ & $0.28 \pm 0.02$ & $0.30 \pm 0.02$ & $0.36 \pm 0.04$ & $0.41 \pm 0.02$ \\
\hline Week 14 & $0.26 \pm 0.01$ & $0.27 \pm 0.02$ & $0.27 \pm 0.02$ & $0.25 \pm 0.02$ & $0.37 \pm 0.02 * *$ & $0.32 \pm 0.03^{*}$ \\
\hline \multicolumn{7}{|c|}{ Mean cell volume (fL) } \\
\hline Day 4 & $58.8 \pm 0.3$ & $58.1 \pm 0.2$ & $58.5 \pm 0.3$ & $57.8 \pm 0.3^{*}$ & $56.9 \pm 0.2 * *$ & $57.5 \pm 0.2 * *$ \\
\hline Day 22 & $57.9 \pm 0.3$ & $58.0 \pm 0.3$ & $57.7 \pm 0.2$ & $57.3 \pm 0.3$ & $56.8 \pm 0.3^{* *}$ & $54.7 \pm 0.2 * *$ \\
\hline Week 14 & $51.2 \pm 0.2$ & $51.6 \pm 0.2$ & $51.3 \pm 0.2$ & $50.8 \pm 0.3$ & $50.5 \pm 0.2 *$ & $49.3 \pm 0.3 * *$ \\
\hline \multicolumn{7}{|c|}{ Mean cell hemoglobin (pg) } \\
\hline Day 4 & $19.7 \pm 0.1$ & $19.5 \pm 0.1$ & $19.6 \pm 0.1$ & $19.5 \pm 0.1$ & $19.1 \pm 0.1 * *$ & $19.3 \pm 0.1 * *$ \\
\hline Day 22 & $19.8 \pm 0.1$ & $19.8 \pm 0.1$ & $19.7 \pm 0.1$ & $19.6 \pm 0.1$ & $19.4 \pm 0.0^{* *}$ & $18.7 \pm 0.1 * *$ \\
\hline Week 14 & $17.0 \pm 0.0$ & $17.2 \pm 0.1$ & $17.1 \pm 0.1$ & $17.1 \pm 0.1$ & $16.9 \pm 0.1$ & $16.5 \pm 0.1 * *$ \\
\hline \multicolumn{7}{|c|}{ Mean cell hemoglobin concentration $(\mathrm{g} / \mathrm{dL})$} \\
\hline Day 4 & $33.6 \pm 0.1$ & $33.6 \pm 0.1$ & $33.5 \pm 0.1$ & $33.7 \pm 0.1$ & $33.6 \pm 0.1$ & $33.5 \pm 0.1$ \\
\hline Day 22 & $34.1 \pm 0.1$ & $34.1 \pm 0.1$ & $34.0 \pm 0.1$ & $34.2 \pm 0.1$ & $34.1 \pm 0.1$ & $34.1 \pm 0.1$ \\
\hline Week 14 & $33.3 \pm 0.1$ & $33.3 \pm 0.1$ & $33.3 \pm 0.1$ & $33.6 \pm 0.1$ & $33.5 \pm 0.1$ & $33.4 \pm 0.1$ \\
\hline \multicolumn{7}{|c|}{ Platelets $\left(10^{3} / \mu \mathrm{L}\right)$} \\
\hline Day 4 & $640.9 \pm 8.6$ & $625.4 \pm 25.0$ & $613.4 \pm 26.7$ & $606.3 \pm 18.3$ & $605.2 \pm 28.6$ & $608.0 \pm 29.7$ \\
\hline Day 22 & $527.2 \pm 21.0$ & $518.5 \pm 21.1$ & $586.0 \pm 19.3$ & $550.9 \pm 18.4$ & $542.0 \pm 22.3$ & $501.9 \pm 22.7$ \\
\hline Week 14 & $418.8 \pm 34.9$ & $443.3 \pm 20.3$ & $501.6 \pm 13.6$ & $419.0 \pm 29.3$ & $485.4 \pm 23.6$ & $503.6 \pm 15.8$ \\
\hline
\end{tabular}


$o$-Chloropyridine, NTP TOX 83

\begin{tabular}{lcccccc}
\hline & $\mathbf{0 ~ p p m}$ & $\mathbf{1 0} \mathbf{~ p p m}$ & $\mathbf{3 0} \mathbf{~ p p m}$ & $\mathbf{1 0 0} \mathbf{~ p p m}$ & $\mathbf{3 0 0} \mathbf{~ p p m}$ & $\mathbf{1 , 0 0 0} \mathbf{~ p p m}$ \\
\hline Leukocytes $\left(10^{3} / \mu \mathrm{L}\right)$ & & & & & & \\
$\quad$ Day 4 & $9.40 \pm 0.21$ & $9.02 \pm 0.25$ & $10.36 \pm 0.29$ & $9.82 \pm 0.50$ & $8.56 \pm 0.36$ & $11.62 \pm 0.37 * *$ \\
Day 22 & $11.68 \pm 0.43$ & $12.03 \pm 0.27$ & $11.85 \pm 0.29$ & $11.26 \pm 0.65$ & $11.83 \pm 0.44$ & $10.68 \pm 0.44$ \\
Week 14 & $10.30 \pm 0.82$ & $11.75 \pm 0.50$ & $12.26 \pm 0.55$ & $10.05 \pm 0.74$ & $10.88 \pm 0.38$ & $11.10 \pm 0.48$ \\
Segmented neutrophils $\left(10^{3} / \mu \mathrm{L}\right)$ & & & & & \\
Day 4 & $0.58 \pm 0.05$ & $0.57 \pm 0.06$ & $0.66 \pm 0.04$ & $0.59 \pm 0.08$ & $0.61 \pm 0.08$ & $0.62 \pm 0.03$ \\
Day 22 & $0.61 \pm 0.05$ & $0.63 \pm 0.03$ & $0.62 \pm 0.05$ & $0.50 \pm 0.04$ & $0.62 \pm 0.03$ & $0.47 \pm 0.03$ \\
Week 14 & $0.56 \pm 0.06$ & $0.76 \pm 0.11$ & $0.72 \pm 0.05$ & $0.56 \pm 0.06$ & $0.55 \pm 0.04$ & $0.49 \pm 0.03$ \\
Lymphocytes $\left(10^{3} / \mu \mathrm{L}\right)$ & & & & & & \\
Day 4 & $8.58 \pm 0.21$ & $8.23 \pm 0.24$ & $9.44 \pm 0.27$ & $8.96 \pm 0.46$ & $7.70 \pm 0.32$ & $10.71 \pm 0.35 * *$ \\
Day 22 & $10.80 \pm 0.37$ & $11.11 \pm 0.23$ & $10.94 \pm 0.24$ & $10.53 \pm 0.58$ & $10.94 \pm 0.43$ & $9.98 \pm 0.42$ \\
Week 14 & $9.43 \pm 0.73$ & $10.51 \pm 0.41$ & $11.17 \pm 0.51^{*}$ & $9.17 \pm 0.68$ & $10.05 \pm 0.33$ & $10.33 \pm 0.43$ \\
Monocytes $\left(10^{3} / \mu \mathrm{L}\right)$ & & & & & & \\
Day 4 & $0.13 \pm 0.01$ & $0.12 \pm 0.01$ & $0.14 \pm 0.02$ & $0.16 \pm 0.02$ & $0.12 \pm 0.01$ & $0.15 \pm 0.01$ \\
Day 22 & $0.13 \pm 0.02$ & $0.13 \pm 0.01$ & $0.14 \pm 0.02$ & $0.10 \pm 0.02$ & $0.13 \pm 0.01$ & $0.12 \pm 0.01$ \\
Week 14 & $0.18 \pm 0.03$ & $0.23 \pm 0.04$ & $0.20 \pm 0.03$ & $0.15 \pm 0.02$ & $0.16 \pm 0.02$ & $0.19 \pm 0.03$ \\
Basophils $\left(10^{3} / \mu \mathrm{L}\right)$ & & & & & & \\
Day 4 & $0.104 \pm 0.007$ & $0.087 \pm 0.006$ & $0.116 \pm 0.013$ & $0.096 \pm 0.006$ & $0.094 \pm 0.008$ & $0.129 \pm 0.010$ \\
Day 22 & $0.134 \pm 0.020$ & $0.144 \pm 0.017$ & $0.132 \pm 0.008$ & $0.106 \pm 0.015$ & $0.125 \pm 0.008$ & $0.102 \pm 0.009$ \\
Week 14 & $0.098 \pm 0.013$ & $0.165 \pm 0.060$ & $0.124 \pm 0.008$ & $0.102 \pm 0.014$ & $0.104 \pm 0.014$ & $0.086 \pm 0.011$ \\
Eosinophils $\left(10^{3} / \mu \mathrm{L}\right)$ & & & & & & \\
Day 4 & $0.01 \pm 0.00$ & $0.02 \pm 0.00$ & $0.02 \pm 0.01$ & $0.02 \pm 0.01$ & $0.03 \pm 0.01$ & $0.01 \pm 0.00$ \\
Day 22 & $0.02 \pm 0.01$ & $0.02 \pm 0.00$ & $0.02 \pm 0.01$ & $0.01 \pm 0.00$ & $0.02 \pm 0.01$ & $0.01 \pm 0.00$ \\
Week 14 & $0.02 \pm 0.00$ & $0.08 \pm 0.04$ & $0.04 \pm 0.01$ & $0.06 \pm 0.03$ & $0.02 \pm 0.00$ & $0.01 \pm 0.00$ \\
\hline Clical Che & & & & & & \\
\hline
\end{tabular}

\section{Clinical Chemistry}

\begin{tabular}{|c|c|c|c|c|c|c|}
\hline $\mathbf{n}$ & 10 & 10 & 10 & 10 & 10 & 10 \\
\hline \multicolumn{7}{|c|}{ Urea nitrogen $(\mathrm{mg} / \mathrm{dL})$} \\
\hline Day 4 & $14.9 \pm 0.6$ & $15.7 \pm 0.7$ & $14.3 \pm 0.4$ & $15.4 \pm 0.6$ & $17.0 \pm 0.7 *$ & $19.6 \pm 0.6^{* *}$ \\
\hline Day 22 & $16.1 \pm 0.5$ & $14.6 \pm 0.4$ & $14.1 \pm 0.5$ & $14.2 \pm 0.5$ & $16.0 \pm 0.7$ & $15.8 \pm 0.6$ \\
\hline Week 14 & $18.5 \pm 0.5$ & $16.7 \pm 0.5$ & $17.5 \pm 0.5$ & $17.2 \pm 0.4$ & $16.6 \pm 0.4$ & $18.4 \pm 0.5$ \\
\hline \multicolumn{7}{|c|}{ Creatinine (mg/dL) } \\
\hline Day 4 & $0.29 \pm 0.01$ & $0.28 \pm 0.01$ & $0.28 \pm 0.01$ & $0.29 \pm 0.01$ & $0.27 \pm 0.02$ & $0.28 \pm 0.01$ \\
\hline Day 22 & $0.33 \pm 0.02$ & $0.31 \pm 0.01$ & $0.34 \pm 0.02$ & $0.34 \pm 0.02$ & $0.31 \pm 0.01$ & $0.28 \pm 0.01$ \\
\hline Week 14 & $0.52 \pm 0.02$ & $0.53 \pm 0.02$ & $0.53 \pm 0.02$ & $0.49 \pm 0.02$ & $0.50 \pm 0.01$ & $0.49 \pm 0.01$ \\
\hline \multicolumn{7}{|c|}{ Total protein $(\mathrm{g} / \mathrm{dL})$} \\
\hline Day 4 & $5.7 \pm 0.1$ & $5.7 \pm 0.0$ & $5.8 \pm 0.1$ & $5.8 \pm 0.1$ & $5.9 \pm 0.1$ & $5.9 \pm 0.1$ \\
\hline Day 22 & $6.4 \pm 0.0$ & $6.4 \pm 0.1$ & $6.5 \pm 0.1$ & $6.5 \pm 0.1$ & $6.8 \pm 0.1^{*}$ & $6.6 \pm 0.1$ \\
\hline Week 14 & $7.3 \pm 0.1$ & $7.3 \pm 0.1$ & $7.5 \pm 0.1$ & $7.8 \pm 0.1^{* *}$ & $8.0 \pm 0.1^{* *}$ & $8.3 \pm 0.1^{* *}$ \\
\hline
\end{tabular}


$o$-Chloropyridine, NTP TOX 83

\begin{tabular}{|c|c|c|c|c|c|c|}
\hline & 0 ppm & $10 \mathrm{ppm}$ & 30 ppm & 100 ppm & 300 ppm & 1,000 ppm \\
\hline \multicolumn{7}{|c|}{ Albumin (g/dL) } \\
\hline Day 4 & $4.1 \pm 0.0$ & $4.1 \pm 0.0$ & $4.1 \pm 0.0$ & $4.1 \pm 0.0$ & $4.2 \pm 0.0$ & $4.3 \pm 0.1 *$ \\
\hline Day 22 & $4.4 \pm 0.0$ & $4.4 \pm 0.1$ & $4.5 \pm 0.1$ & $4.6 \pm 0.0 * *$ & $4.7 \pm 0.1 * *$ & $4.7 \pm 0.1 * *$ \\
\hline Week 14 & $4.9 \pm 0.0$ & $4.9 \pm 0.0$ & $5.0 \pm 0.0^{*}$ & $5.2 \pm 0.1 * *$ & $5.4 \pm 0.1 * *$ & $5.7 \pm 0.0 * *$ \\
\hline \multicolumn{7}{|c|}{ Alanine aminotransferase (IU/L) } \\
\hline Day 4 & $51 \pm 2$ & $50 \pm 1$ & $53 \pm 1$ & $50 \pm 2$ & $55 \pm 2$ & $46 \pm 2$ \\
\hline Day 22 & $44 \pm 1$ & $41 \pm 1$ & $41 \pm 1$ & $39 \pm 1 * *$ & $38 \pm 1 * *$ & $44 \pm 1$ \\
\hline Week 14 & $76 \pm 6$ & $72 \pm 5$ & $70 \pm 4$ & $56 \pm 3 * *$ & $40 \pm 2 * *$ & $42 \pm 2 * *$ \\
\hline \multicolumn{7}{|c|}{ Alkaline phosphatase (IU/L) } \\
\hline Day 4 & $626 \pm 13$ & $622 \pm 8$ & $626 \pm 12$ & $622 \pm 9$ & $657 \pm 10$ & $701 \pm 15^{* *}$ \\
\hline Day 22 & $476 \pm 8$ & $486 \pm 11$ & $476 \pm 9$ & $441 \pm 10^{*}$ & $392 \pm 8 * *$ & $392 \pm 10 * *$ \\
\hline Week 14 & $251 \pm 9$ & $234 \pm 5$ & $186 \pm 16^{* *}$ & $218 \pm 4^{* *}$ & $198 \pm 4^{* *}$ & $195 \pm 3 * *$ \\
\hline \multicolumn{7}{|c|}{ Creatine kinase (IU/L) } \\
\hline Day 4 & $305 \pm 39$ & $270 \pm 28$ & $268 \pm 20$ & $553 \pm 211$ & $440 \pm 51$ & $386 \pm 33$ \\
\hline Day 22 & $324 \pm 74$ & $214 \pm 35$ & $235 \pm 31$ & $209 \pm 31$ & $190 \pm 27$ & $247 \pm 33$ \\
\hline Week 14 & $219 \pm 36$ & $196 \pm 18^{\mathrm{b}}$ & $177 \pm 18$ & $262 \pm 52$ & $199 \pm 31$ & $174 \pm 16$ \\
\hline \multicolumn{7}{|c|}{ Sorbitol dehydrogenase (IU/L) } \\
\hline Day 4 & $13 \pm 1$ & $11 \pm 1$ & $11 \pm 1$ & $10 \pm 2$ & $13 \pm 1$ & $16 \pm 1$ \\
\hline Day 22 & $15 \pm 1$ & $13 \pm 1$ & $17 \pm 1$ & $14 \pm 1$ & $16 \pm 1$ & $23 \pm 1 * *$ \\
\hline Week 14 & $33 \pm 2$ & $32 \pm 2$ & $30 \pm 1$ & $32 \pm 2$ & $30 \pm 2$ & $33 \pm 2$ \\
\hline \multicolumn{7}{|c|}{ Bile acids $(\mu \mathrm{mol} / \mathrm{L})$} \\
\hline Day 4 & $23.8 \pm 1.4$ & $26.5 \pm 2.1$ & $26.4 \pm 1.3$ & $33.8 \pm 2.2 * *$ & $49.8 \pm 6.4^{* *}$ & $41.0 \pm 5.4 * *$ \\
\hline Day 22 & $25.7 \pm 1.1$ & $25.5 \pm 1.2$ & $31.0 \pm 2.6$ & $28.0 \pm 2.0$ & $34.9 \pm 2.9 * *$ & $39.1 \pm 2.8 * *$ \\
\hline Week 14 & $31.1 \pm 3.4$ & $26.0 \pm 1.0$ & $29.0 \pm 1.9$ & $26.1 \pm 0.8$ & $33.1 \pm 3.1$ & $39.2 \pm 2.9$ \\
\hline
\end{tabular}

\section{Female}

\section{Hematology}

n

\begin{tabular}{lcccccc} 
Day 4 & 10 & 9 & 10 & 10 & 10 & 9 \\
Day 22 & 10 & 10 & 10 & 10 & 10 & 10 \\
Week 14 & 10 & 10 & 10 & 10 & 10 & 10 \\
\hline
\end{tabular}

Hematocrit (\%)

Day 4

Day 22

Week 14

Hemoglobin (g/dL)

Day 4

Day 22

Week 14
$42.6 \pm 0.5$

$44.9 \pm 0.3$

$44.3 \pm 0.8$

$14.4 \pm 0.2$

$14.1 \pm 0.2$

$15.9 \pm 0.1$

$15.4 \pm 0.1$

$45.9 \pm 0.4$

$45.2 \pm 0.3$

$15.5 \pm 0.1$

$15.1 \pm 0.2$
$42.6 \pm 0.4$

$44.4 \pm 0.4$

$45.1 \pm 0.3$

$40.6 \pm 1.1$

$43.5 \pm 0.3^{*}$

$43.7 \pm 0.7$

$39.8 \pm 0.8^{*}$

$44.9 \pm 0.8$

$42.4 \pm 0.6^{* *}$

$42.4 \pm 1.1^{* *}$

$14.4 \pm 0.2$

$13.8 \pm 0.4$

$13.6 \pm 0.3$

$15.3 \pm 0.3$

$15.4 \pm 0.1$

$15.2 \pm 0.1$

$14.8 \pm 0.2^{*}$

$14.7 \pm 0.4 * *$

$15.4 \pm 0.1$

$15.0 \pm 0.2$

$14.7 \pm 0.1 *$

$13.9 \pm 0.2^{* *}$ 
$o$-Chloropyridine, NTP TOX 83

\begin{tabular}{|c|c|c|c|c|c|c|}
\hline & 0 ppm & $10 \mathrm{ppm}$ & 30 ppm & 100 ppm & 300 ppm & 1,000 ppm \\
\hline \multicolumn{7}{|c|}{ Erythrocytes $\left(10^{6} / \mu \mathrm{L}\right)$} \\
\hline Day 4 & $7.33 \pm 0.09$ & $7.16 \pm 0.09$ & $7.35 \pm 0.07$ & $7.02 \pm 0.19$ & $6.93 \pm 0.15$ & $7.87 \pm 0.13$ \\
\hline Day 22 & $7.80 \pm 0.07$ & $7.95 \pm 0.08$ & $7.75 \pm 0.08$ & $7.70 \pm 0.06$ & $7.51 \pm 0.13$ & $7.74 \pm 0.17$ \\
\hline Week 14 & $8.30 \pm 0.13$ & $8.50 \pm 0.05$ & $8.48 \pm 0.07$ & $8.27 \pm 0.12$ & $8.17 \pm 0.03$ & $7.90 \pm 0.07 * *$ \\
\hline \multicolumn{7}{|c|}{ Reticulocytes $\left(10^{6} / \mu \mathrm{L}\right)$} \\
\hline Day 4 & $0.36 \pm 0.01$ & $0.35 \pm 0.03$ & $0.35 \pm 0.01$ & $0.36 \pm 0.02$ & $0.32 \pm 0.02$ & $0.28 \pm 0.01^{* *}$ \\
\hline Day 22 & $0.20 \pm 0.01$ & $0.20 \pm 0.01$ & $0.20 \pm 0.01$ & $0.19 \pm 0.01$ & $0.22 \pm 0.03$ & $0.29 \pm 0.02 *$ \\
\hline Week 14 & $0.23 \pm 0.02$ & $0.26 \pm 0.02$ & $0.21 \pm 0.02$ & $0.23 \pm 0.02$ & $0.26 \pm 0.03$ & $0.33 \pm 0.02 * *$ \\
\hline \multicolumn{7}{|c|}{ Mean cell volume (fL) } \\
\hline Day 4 & $58.0 \pm 0.2$ & $57.8 \pm 0.2$ & $57.8 \pm 0.3$ & $57.8 \pm 0.3$ & $57.6 \pm 0.2$ & $57.1 \pm 0.2 * *$ \\
\hline Day 22 & $57.5 \pm 0.2$ & $57.7 \pm 0.2$ & $57.4 \pm 0.3$ & $56.6 \pm 0.2 *$ & $56.6 \pm 0.4^{*}$ & $54.6 \pm 0.2 * *$ \\
\hline Week 14 & $53.5 \pm 0.2$ & $53.2 \pm 0.1$ & $53.2 \pm 0.1$ & $52.8 \pm 0.1 * *$ & $52.1 \pm 0.2 * *$ & $51.5 \pm 0.2 * *$ \\
\hline \multicolumn{7}{|c|}{ Mean cell hemoglobin (pg) } \\
\hline Day 4 & $19.7 \pm 0.1$ & $19.7 \pm 0.1$ & $19.6 \pm 0.1$ & $19.7 \pm 0.1$ & $19.7 \pm 0.1$ & $19.4 \pm 0.1$ \\
\hline Day 22 & $19.9 \pm 0.1$ & $20.0 \pm 0.1$ & $19.9 \pm 0.1$ & $19.8 \pm 0.1$ & $19.7 \pm 0.1$ & $19.0 \pm 0.1 * *$ \\
\hline Week 14 & $18.2 \pm 0.1$ & $18.1 \pm 0.1$ & $18.1 \pm 0.1$ & $18.1 \pm 0.1$ & $18.0 \pm 0.1$ & $17.6 \pm 0.1 * *$ \\
\hline \multicolumn{7}{|c|}{ Mean cell hemoglobin concentration $(\mathrm{g} / \mathrm{dL})$} \\
\hline Day 4 & $33.8 \pm 0.1$ & $34.0 \pm 0.1$ & $33.8 \pm 0.1$ & $34.1 \pm 0.1$ & $34.3 \pm 0.1^{*}$ & $34.0 \pm 0.1$ \\
\hline Day 22 & $34.5 \pm 0.1$ & $34.6 \pm 0.1$ & $34.7 \pm 0.1$ & $35.0 \pm 0.1^{*}$ & $34.8 \pm 0.1$ & $34.7 \pm 0.1$ \\
\hline Week 14 & $34.1 \pm 0.2$ & $34.1 \pm 0.1$ & $34.1 \pm 0.1$ & $34.4 \pm 0.1$ & $34.5 \pm 0.2$ & $34.0 \pm 0.1$ \\
\hline \multicolumn{7}{|c|}{ Platelets $\left(10^{3} / \mu \mathrm{L}\right)$} \\
\hline Day 4 & $491.3 \pm 17.4$ & $493.4 \pm 26.1$ & $474.1 \pm 18.2$ & $530.4 \pm 19.1$ & $477.7 \pm 22.1$ & $520.8 \pm 31.1$ \\
\hline Day 22 & $457.9 \pm 30.4$ & $519.9 \pm 15.7$ & $494.0 \pm 22.7$ & $493.1 \pm 13.2$ & $466.1 \pm 20.9$ & $450.5 \pm 25.3$ \\
\hline Week 14 & $464.9 \pm 19.1^{\mathrm{b}}$ & $460.9 \pm 10.7$ & $519.1 \pm 23.4$ & $467.5 \pm 25.0$ & $481.8 \pm 27.0$ & $513.1 \pm 21.5$ \\
\hline \multicolumn{7}{|c|}{ Leukocytes $\left(10^{3} / \mu \mathrm{L}\right)$} \\
\hline Day 4 & $10.23 \pm 0.27$ & $9.24 \pm 0.57$ & $9.40 \pm 0.62$ & $9.09 \pm 0.79$ & $9.68 \pm 0.68$ & $12.54 \pm 0.43 *$ \\
\hline Day 22 & $12.19 \pm 0.36$ & $12.38 \pm 0.32$ & $11.45 \pm 0.38$ & $11.81 \pm 0.41$ & $11.51 \pm 0.44$ & $11.40 \pm 0.55$ \\
\hline Week 14 & $9.17 \pm 0.53$ & $9.29 \pm 0.29$ & $9.15 \pm 0.52$ & $9.65 \pm 0.47$ & $9.08 \pm 0.69$ & $9.74 \pm 0.43$ \\
\hline \multicolumn{7}{|c|}{ Segmented neutrophils $\left(10^{3} / \mu \mathrm{L}\right)$} \\
\hline Day 4 & $0.61 \pm 0.03$ & $0.55 \pm 0.05$ & $0.60 \pm 0.06$ & $0.49 \pm 0.05$ & $0.52 \pm 0.05$ & $0.68 \pm 0.05$ \\
\hline Day 22 & $0.66 \pm 0.03$ & $0.63 \pm 0.03$ & $0.69 \pm 0.06$ & $0.56 \pm 0.04$ & $0.51 \pm 0.03^{* *}$ & $0.52 \pm 0.06^{*}$ \\
\hline Week 14 & $0.54 \pm 0.03$ & $0.67 \pm 0.06$ & $0.74 \pm 0.09$ & $0.52 \pm 0.03$ & $0.61 \pm 0.08$ & $0.58 \pm 0.07$ \\
\hline \multicolumn{7}{|c|}{ Lymphocytes $\left(10^{3} / \mu \mathrm{L}\right)$} \\
\hline Day 4 & $9.37 \pm 0.24$ & $8.49 \pm 0.50$ & $8.52 \pm 0.55$ & $8.36 \pm 0.72$ & $8.91 \pm 0.62$ & $11.49 \pm 0.38$ \\
\hline Day 22 & $11.23 \pm 0.32$ & $11.47 \pm 0.28$ & $10.45 \pm 0.31$ & $11.03 \pm 0.36$ & $10.75 \pm 0.40$ & $10.66 \pm 0.49$ \\
\hline Week 14 & $8.42 \pm 0.51$ & $8.40 \pm 0.28$ & $8.12 \pm 0.42$ & $8.89 \pm 0.45$ & $8.23 \pm 0.63$ & $8.96 \pm 0.39$ \\
\hline \multicolumn{7}{|c|}{ Monocytes $\left(10^{3} / \mu \mathrm{L}\right)$} \\
\hline Day 4 & $0.12 \pm 0.01$ & $0.11 \pm 0.02$ & $0.14 \pm 0.02$ & $0.12 \pm 0.01$ & $0.13 \pm 0.02$ & $0.17 \pm 0.02$ \\
\hline Day 22 & $0.13 \pm 0.02$ & $0.13 \pm 0.01$ & $0.13 \pm 0.02$ & $0.09 \pm 0.01$ & $0.09 \pm 0.01$ & $0.09 \pm 0.01$ \\
\hline
\end{tabular}




\begin{tabular}{lcccccc}
\hline & $\mathbf{0 ~ p p m}$ & $\mathbf{1 0} \mathbf{~ p p m}$ & $\mathbf{3 0} \mathbf{~ p p m}$ & $\mathbf{1 0 0} \mathbf{~ p p m}$ & $\mathbf{3 0 0} \mathbf{~ p p m}$ & $\mathbf{1 , 0 0 0} \mathbf{~ p p m}$ \\
\hline Week 14 & $0.13 \pm 0.02$ & $0.14 \pm 0.02$ & $0.17 \pm 0.02$ & $0.15 \pm 0.02$ & $0.15 \pm 0.03$ & $0.14 \pm 0.01$ \\
Basophils $\left(10^{3} / \mu \mathrm{L}\right)$ & & & & & & \\
$\quad$ Day 4 & $0.113 \pm 0.007$ & $0.096 \pm 0.010$ & $0.117 \pm 0.019$ & $0.102 \pm 0.011$ & $0.124 \pm 0.015$ & $0.184 \pm 0.015^{* *}$ \\
Day 22 & $0.147 \pm 0.013$ & $0.138 \pm 0.009$ & $0.164 \pm 0.028$ & $0.116 \pm 0.013$ & $0.127 \pm 0.016$ & $0.117 \pm 0.011$ \\
Week 14 & $0.073 \pm 0.018$ & $0.065 \pm 0.007$ & $0.085 \pm 0.023$ & $0.067 \pm 0.006$ & $0.061 \pm 0.012$ & $0.058 \pm 0.005$ \\
Eosinophils $\left(10^{3} / \mu \mathrm{L}\right)$ & & & & & & \\
Day 4 & $0.02 \pm 0.00$ & $0.01 \pm 0.00$ & $0.02 \pm 0.01$ & $0.02 \pm 0.00$ & $0.02 \pm 0.00$ & $0.02 \pm 0.00$ \\
Day 22 & $0.02 \pm 0.00$ & $0.02 \pm 0.00$ & $0.02 \pm 0.01$ & $0.01 \pm 0.00$ & $0.02 \pm 0.00$ & $0.01 \pm 0.00$ \\
Week 14 & $0.02 \pm 0.01$ & $0.02 \pm 0.01$ & $0.03 \pm 0.01$ & $0.02 \pm 0.00$ & $0.02 \pm 0.01$ & $0.02 \pm 0.00$ \\
\hline
\end{tabular}

\section{Clinical Chemistry}

10

10

10

10

10

Urea nitrogen $(\mathrm{mg} / \mathrm{dL})$

$\begin{array}{lcccccc}\text { Day } 4 & 14.1 \pm 0.3 & 13.7 \pm 0.8 & 13.5 \pm 0.9 & 14.3 \pm 0.6 & 15.4 \pm 0.8 & 16.7 \pm 0.6^{* *} \\ \text { Day 22 } & 18.9 \pm 0.6 & 18.9 \pm 0.8 & 18.3 \pm 0.7 & 18.9 \pm 0.6 & 18.8 \pm 0.9 & 19.4 \pm 0.7 \\ \text { Week 14 } & 18.3 \pm 0.8 & 18.9 \pm 0.5 & 20.0 \pm 0.7 & 18.0 \pm 0.7 & 18.5 \pm 0.5 & 17.5 \pm 0.6\end{array}$

Creatinine (mg/dL)

Day 4

$0.30 \pm 0.00$

$0.29 \pm 0.01$

$0.32 \pm 0.01$

$0.30 \pm 0.01$

$0.30 \pm 0.00$

$0.27 \pm 0.02$

Day 22

$0.38 \pm 0.01$

$0.43 \pm 0.02$

$0.37 \pm 0.02$

$0.38 \pm 0.01$

$0.38 \pm 0.01$

$0.32 \pm 0.02$

Week 14

$0.51 \pm 0.02$

$0.51 \pm 0.01$

$0.58 \pm 0.01 * \quad 0.52 \pm 0.01$

$0.49 \pm 0.02$

$0.48 \pm 0.01$

Total protein $(\mathrm{g} / \mathrm{dL})$

Day 4

$5.8 \pm 0.0$

$6.0 \pm 0.1$

$5.9 \pm 0.0$

$5.9 \pm 0.1$

$5.8 \pm 0.1$

Day 22

$6.3 \pm 0.1$

$6.6 \pm 0.1$

$6.5 \pm 0.2$

$6.5 \pm 0.1$

$6.5 \pm 0.1$

$6.6 \pm 0.1$

Week 14

$6.9 \pm 0.1$

$7.2 \pm 0.1$

$7.5 \pm 0.1 * *$

$7.4 \pm 0.1 * *$

$7.5 \pm 0.1 * *$

$7.8 \pm 0.1 * *$

Albumin (g/dL)

Day 4

$$
4.3 \pm 0.0
$$

$4.3 \pm 0.0$

$4.5 \pm 0.0^{*}$

$4.3 \pm 0.0$

$4.3 \pm 0.0$

$4.4 \pm 0.1$

Day 22

$4.6 \pm 0.1$

$4.7 \pm 0.0$

$4.7 \pm 0.1$

$4.8 \pm 0.1^{* *}$

$4.8 \pm 0.1^{*}$

$4.9 \pm 0.1 * *$

Week 14

$5.1 \pm 0.2$

$5.1 \pm 0.1$

$5.3 \pm 0.1 *$

$5.4 \pm 0.1 * *$

$5.4 \pm 0.1^{* *}$

$5.7 \pm 0.1 * *$

Alanine aminotransferase (IU/L)

Day 4

$$
46 \pm 1
$$

Day 22

$35 \pm 1$

$45 \pm 1$

$48 \pm 2$

$$
47 \pm 1
$$

$50 \pm 1$

$44 \pm 2$

Week 14

$72 \pm 6$

$38 \pm 1$

$37 \pm 2$

$36 \pm 1$

$34 \pm 1$

$35 \pm 1$

$75 \pm 9$

$85 \pm 8$

$45 \pm 2 * *$

$43 \pm 2 * *$

$31 \pm 1 * *$

Alkaline phosphatase (IU/L)

\section{Day 4}

Day 22

Week 14

$541 \pm 8$

$416 \pm 9$

$207 \pm 10$

Creatine kinase (IU/L)

$$
\text { Day } 4
$$

Day 22

$270 \pm 44$

$275 \pm 39$

$544 \pm 5$

$557 \pm 9$

$421 \pm 9$

$399 \pm 8$

$188 \pm 5$

$177 \pm 3^{* *}$

$255 \pm 32 \quad 298 \pm 47^{\mathrm{b}}$

$292 \pm 59 \quad 182 \pm 26$

$510 \pm 156$

$388 \pm 37 \quad 362 \pm 57$

Week 14
$554 \pm 9$

$567 \pm 19$

$337 \pm 13 * *$

$575 \pm 21$

$392 \pm 7$

$165 \pm 4 * *$

$170 \pm 10 * *$

$356 \pm 13 * *$

$146 \pm 3^{* *}$

$\begin{array}{lll}321 \pm 55 & 272 \pm 27 & 374 \pm 35 \\ 227 \pm 58 & 366 \pm 77 & 302 \pm 69 \\ 328 \pm 74 & 526 \pm 159 & 281 \pm 42\end{array}$


$o$-Chloropyridine, NTP TOX 83

\begin{tabular}{lcccccc}
\hline & $\mathbf{0 ~} \mathbf{~ p p m}$ & $\mathbf{1 0} \mathbf{~ p p m}$ & $\mathbf{3 0} \mathbf{~ p p m}$ & $\mathbf{1 0 0} \mathbf{~ p p m}$ & $\mathbf{3 0 0} \mathbf{~ p p m}$ & $\mathbf{1 , 0 0 0} \mathbf{~ p p m}$ \\
\hline \multicolumn{2}{l}{ Sorbitol dehydrogenase (IU/L) } & & & & & \\
$\quad$ Day 4 & $14 \pm 1$ & $15 \pm 1$ & $16 \pm 1$ & $15 \pm 1$ & $16 \pm 0$ & $16 \pm 1$ \\
Day 22 & $19 \pm 2$ & $17 \pm 3$ & $20 \pm 2$ & $20 \pm 3$ & $19 \pm 3$ & $18 \pm 2$ \\
Week 14 & $35 \pm 3$ & $38 \pm 1$ & $41 \pm 1$ & $35 \pm 3$ & $34 \pm 2$ & $35 \pm 2$ \\
Bile acids $(\mu \mathrm{mol} / \mathrm{L})$ & & & & & & \\
Day 4 & $26.4 \pm 1.3$ & $24.6 \pm 0.8$ & $27.3 \pm 2.2$ & $26.9 \pm 1.6$ & $40.3 \pm 3.8^{* *}$ & $31.7 \pm 2.8^{*}$ \\
Day 22 & $24.0 \pm 0.8$ & $30.1 \pm 1.5^{*}$ & $27.0 \pm 1.4$ & $23.1 \pm 1.1$ & $29.1 \pm 1.7$ & $27.2 \pm 1.0$ \\
Week 14 & $27.0 \pm 1.7$ & $34.3 \pm 2.9$ & $32.6 \pm 1.4$ & $34.8 \pm 3.3$ & $30.8 \pm 1.5$ & $36.4 \pm 3.2^{*}$ \\
\hline
\end{tabular}

*Significantly different $(\mathrm{P} \leq 0.05)$ from the control group by Dunn's or Shirley's test.

$* * \mathrm{P} \leq 0.01$.

${ }^{a}$ Data are presented as mean \pm standard error. Statistical tests were performed on unrounded data. ${ }^{b} \mathrm{n}=9$. 
$o$-Chloropyridine, NTP TOX 83

Table B-2. Hematology Data for Mice in the Three-month Drinking Water Study of $o$-Chloropyridine ${ }^{\text {a }}$

\begin{tabular}{|c|c|c|c|c|c|c|}
\hline & 0 ppm & 10 ppm & 30 ppm & 100 ppm & 300 ppm & 1,000 ppm \\
\hline \multicolumn{7}{|l|}{ Male } \\
\hline $\mathbf{n}$ & 10 & 10 & 10 & 10 & 10 & 10 \\
\hline Hematocrit (\%) & $51.2 \pm 0.4$ & $51.4 \pm 0.5$ & $50.4 \pm 0.7$ & $50.2 \pm 0.4$ & $50.3 \pm 0.6$ & $50.5 \pm 0.6$ \\
\hline Hemoglobin (g/dL) & $16.4 \pm 0.1$ & $16.5 \pm 0.1$ & $16.1 \pm 0.2$ & $16.1 \pm 0.1$ & $16.2 \pm 0.2$ & $16.2 \pm 0.2$ \\
\hline Erythrocytes $\left(10^{6} / \mu \mathrm{L}\right)$ & $10.63 \pm 0.09$ & $10.78 \pm 0.10$ & $10.46 \pm 0.13$ & $10.65 \pm 0.07$ & $10.67 \pm 0.11$ & $10.69 \pm 0.08$ \\
\hline Reticulocytes $\left(10^{6} / \mu \mathrm{L}\right)$ & $0.25 \pm 0.02$ & $0.27 \pm 0.02$ & $0.29 \pm 0.03$ & $0.28 \pm 0.02$ & $0.28 \pm 0.03$ & $0.26 \pm 0.03$ \\
\hline Mean cell volume (fL) & $47.3 \pm 0.3$ & $47.3 \pm 0.3$ & $47.5 \pm 0.2$ & $46.8 \pm 0.1$ & $47.0 \pm 0.4$ & $46.7 \pm 0.2$ \\
\hline $\begin{array}{l}\text { Mean cell hemoglobin } \\
\quad(p g)\end{array}$ & $15.2 \pm 0.1$ & $15.2 \pm 0.1$ & $15.2 \pm 0.1$ & $15.1 \pm 0.1$ & $15.1 \pm 0.1$ & $14.9 \pm 0.1$ \\
\hline $\begin{array}{l}\text { Mean cell hemoglobin } \\
\text { concentration }(\mathrm{g} / \mathrm{dL})\end{array}$ & $32.1 \pm 0.2$ & $32.1 \pm 0.1$ & $32.0 \pm 0.1$ & $32.2 \pm 0.1$ & $32.2 \pm 0.1$ & $32.1 \pm 0.1$ \\
\hline Platelets $\left(10^{3} / \mu \mathrm{L}\right)$ & $585.6 \pm 24.8$ & $569.5 \pm 15.9$ & $580.9 \pm 17.8$ & $572.3 \pm 17.3$ & $549.7 \pm 21.3$ & $549.8 \pm 17.9$ \\
\hline Leukocytes $\left(10^{3} / \mu \mathrm{L}\right)$ & $6.13 \pm 0.35$ & $6.39 \pm 0.56$ & $5.91 \pm 0.34$ & $6.07 \pm 0.31$ & $5.88 \pm 0.28$ & $5.95 \pm 0.39$ \\
\hline $\begin{array}{l}\text { Segmented neutrophils } \\
\left(10^{3} / \mu \mathrm{L}\right)\end{array}$ & $0.25 \pm 0.02$ & $0.28 \pm 0.05$ & $0.25 \pm 0.03$ & $0.28 \pm 0.03$ & $0.26 \pm 0.03$ & $0.27 \pm 0.04$ \\
\hline Lymphocytes $\left(10^{3} / \mu \mathrm{L}\right)$ & $5.68 \pm 0.33$ & $5.86 \pm 0.49$ & $5.48 \pm 0.30$ & $5.56 \pm 0.29$ & $5.39 \pm 0.29$ & $5.44 \pm 0.34$ \\
\hline Monocytes $\left(10^{3} / \mu \mathrm{L}\right)$ & $0.14 \pm 0.01$ & $0.15 \pm 0.01$ & $0.15 \pm 0.02$ & $0.14 \pm 0.01$ & $0.17 \pm 0.05$ & $0.14 \pm 0.02$ \\
\hline Basophils $\left(10^{3} / \mu \mathrm{L}\right)$ & $0.031 \pm 0.006$ & $0.065 \pm 0.025$ & $0.033 \pm 0.005$ & $0.041 \pm 0.005$ & $0.034 \pm 0.004$ & $0.067 \pm 0.023$ \\
\hline Eosinophils $\left(10^{3} / \mu \mathrm{L}\right)$ & $0.02 \pm 0.00$ & $0.04 \pm 0.02$ & $0.02 \pm 0.00$ & $0.03 \pm 0.00$ & $0.02 \pm 0.00$ & $0.04 \pm 0.01$ \\
\hline
\end{tabular}

Female

\begin{tabular}{|c|c|c|c|c|c|c|}
\hline $\mathbf{n}$ & 9 & 10 & 10 & 10 & 10 & 10 \\
\hline Hematocrit (\%) & $50.9 \pm 1.1$ & $50.5 \pm 0.7$ & $48.9 \pm 0.4$ & $50.5 \pm 0.4$ & $48.8 \pm 0.6$ & $46.6 \pm 0.4$ \\
\hline Hemoglobin (g/dL) & $16.2 \pm 0.3$ & $16.0 \pm 0.2$ & $15.7 \pm 0.1$ & $16.2 \pm 0.1$ & $15.7 \pm 0.2$ & $15.1 \pm 0.1 * *$ \\
\hline Erythrocytes $\left(10^{6} / \mu \mathrm{L}\right)$ & $10.56 \pm 0.20$ & $10.42 \pm 0.12$ & $10.23 \pm 0.09$ & $10.54 \pm 0.07$ & $10.22 \pm 0.13$ & $9.96 \pm 0.09 * *$ \\
\hline Reticulocytes $\left(10^{6} / \mu \mathrm{L}\right)$ & $0.40 \pm 0.03$ & $0.33 \pm 0.03$ & $0.32 \pm 0.03$ & $0.37 \pm 0.03$ & $0.37 \pm 0.03$ & $0.31 \pm 0.03$ \\
\hline Mean cell volume (fL) & $48.2 \pm 0.2$ & $48.2 \pm 0.1$ & $47.9 \pm 0.2$ & $47.9 \pm 0.2$ & $47.7 \pm 0.2$ & $46.9 \pm 0.2 * *$ \\
\hline $\begin{array}{l}\text { Mean cell hemoglobin } \\
\quad(\mathrm{pg})\end{array}$ & $15.3 \pm 0.1$ & $15.3 \pm 0.0$ & $15.3 \pm 0.1$ & $15.4 \pm 0.1$ & $15.4=$ & $15.2 \pm 0.1$ \\
\hline $\begin{array}{l}\text { Mean cell hemoglobin } \\
\text { concentration }(\mathrm{g} / \mathrm{dL})\end{array}$ & 0.1 & $.7 \pm 0.1$ & $.0 \pm 0.1$ & $32.0 \pm 0.1$ & 0.1 & $.4 \pm 0.1 * *$ \\
\hline Platelets $\left(10^{3} / \mu \mathrm{L}\right)$ & $461.3 \pm 32.5$ & $485.2 \pm 28.8$ & $518.3 \pm 26.1$ & $491.6 \pm 24.8$ & $552.1 \pm 20.5^{*}$ & $569.4 \pm 23.2 *$ \\
\hline Leukocytes $\left(10^{3} / \mu \mathrm{L}\right)$ & $4.18 \pm 0.41$ & $4.10 \pm 0.24$ & $4.07 \pm 0.27$ & $4.22 \pm 0.31$ & $4.16 \pm 0.19$ & $4.56 \pm 0.27$ \\
\hline $\begin{array}{l}\text { Segmented neutrophils } \\
\left(10^{3} / \mu \mathrm{L}\right)\end{array}$ & $0.21 \pm 0.04$ & $0.14 \pm 0.02$ & $0.21 \pm 0.07$ & $0.16 \pm 0.02$ & $0.13 \pm 0.01$ & $0.15 \pm 0.02$ \\
\hline Lymphocytes $\left(10^{3} / \mu \mathrm{L}\right)$ & $3.80 \pm 0.34$ & $3.87 \pm 0.23$ & $3.73 \pm 0.22$ & $3.96 \pm 0.29$ & $3.93 \pm 0.19$ & $4.31 \pm 0.27$ \\
\hline Monocytes $\left(10^{3} / \mu \mathrm{L}\right)$ & $0.08 \pm 0.01$ & $0.09 \pm 0.01$ & $0.09 \pm 0.02$ & $0.08 \pm 0.01$ & $0.08 \pm 0.01$ & $0.09 \pm 0.01$ \\
\hline Basophils $\left(10^{3} / \mu \mathrm{L}\right)$ & $0.050 \pm 0.017$ & $0.019 \pm 0.006$ & $0.020 \pm 0.007$ & $0.024 \pm 0.008$ & $0.019 \pm 0.007$ & $0.011 \pm 0.002$ \\
\hline Eosinophils $\left(10^{3} / \mu \mathrm{L}\right)$ & $0.03 \pm 0.01$ & $0.01 \pm 0.00$ & $0.01 \pm 0.00$ & $0.01 \pm 0.01$ & $0.01 \pm 0.00$ & $0.01 \pm 0.00$ \\
\hline
\end{tabular}

*Significantly different $(\mathrm{P} \leq 0.05)$ from the control group by Shirley’s test.

$* * \mathrm{P} \leq 0.01$.

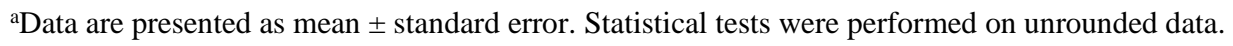




\section{Appendix C. Organ Weights and Organ-Weight-to-Body- Weight Ratios}

\section{Tables}

Table C-1. Organ Weights and Organ-Weight-to-Body-Weight Ratios for Rats in the Two-week Dermal Study of $o$-Chloropyridine

Table C-2. Organ Weights and Organ-Weight-to-Body-Weight Ratios for Rats in the Three-month Drinking Water Study of $o$-Chloropyridine.

Table C-3. Organ Weights and Organ-Weight-to-Body-Weight Ratios for Mice in the Two-week Dermal Study of $o$-Chloropyridine

Table C-4. Organ Weights and Organ-Weight-to-Body-Weight Ratios for Mice in the Three-month Drinking Water Study of $o$-Chloropyridine. C-6 
Table C-1. Organ Weights and Organ-Weight-to-Body-Weight Ratios for Rats in the Two-week Dermal Study of $o$-Chloropyridine ${ }^{\text {a }}$

\begin{tabular}{|c|c|c|c|c|c|c|}
\hline & $\begin{array}{l}\text { Vehicle } \\
\text { Control }\end{array}$ & $6.25 \mathrm{mg} / \mathrm{kg}$ & $12.5 \mathrm{mg} / \mathrm{kg}$ & $25 \mathrm{mg} / \mathrm{kg}$ & $50 \mathrm{mg} / \mathrm{kg}$ & $100 \mathrm{mg} / \mathrm{kg}$ \\
\hline n & 5 & 5 & 5 & 5 & 5 & 5 \\
\hline \multicolumn{7}{|l|}{ Male } \\
\hline Necropsy body wt & $157 \pm 6$ & $159 \pm 6$ & $157 \pm 4$ & $153 \pm 3$ & $158 \pm 5$ & $158 \pm 4$ \\
\hline \multicolumn{7}{|l|}{ Heart } \\
\hline Absolute & $0.60 \pm 0.02$ & $0.61 \pm 0.02$ & $0.59 \pm 0.02$ & $0.60 \pm 0.01$ & $0.65 \pm 0.04$ & $0.60 \pm 0.01$ \\
\hline Relative & $3.819 \pm 0.079$ & $3.829 \pm 0.074$ & $3.752 \pm 0.080$ & $3.920 \pm 0.045$ & $4.131 \pm 0.250$ & $3.823 \pm 0.100$ \\
\hline \multicolumn{7}{|l|}{ R. Kidney } \\
\hline Absolute & $0.69 \pm 0.03$ & $0.71 \pm 0.03$ & $0.72 \pm 0.02$ & $0.68 \pm 0.03$ & $0.73 \pm 0.03$ & $0.73 \pm 0.02$ \\
\hline Relative & $4.408 \pm 0.089$ & $4.439 \pm 0.073$ & $4.572 \pm 0.059$ & $4.436 \pm 0.152$ & $4.623 \pm 0.084$ & $4.604 \pm 0.104$ \\
\hline \multicolumn{7}{|l|}{ Liver } \\
\hline Absolute & $8.02 \pm 0.28$ & $8.13 \pm 0.18$ & $8.25 \pm 0.23$ & $8.03 \pm 0.16$ & $8.87 \pm 0.35^{*}$ & $8.79 \pm 0.11 *$ \\
\hline Relative & $51.083 \pm 0.188$ & $51.251 \pm 0.857$ & $52.473 \pm 1.018$ & $52.428 \pm 0.422$ & $55.961 \pm 0.567^{* *}$ & $55.732 \pm 0.777 * *$ \\
\hline \multicolumn{7}{|l|}{ Lung } \\
\hline Absolute & $1.00 \pm 0.05$ & $1.13 \pm 0.16$ & $1.06 \pm 0.06$ & $0.98 \pm 0.04$ & $1.09 \pm 0.07$ & $0.79 \pm 0.18$ \\
\hline Relative & $6.353 \pm 0.107$ & $7.001 \pm 0.731$ & $6.725 \pm 0.277$ & $6.413 \pm 0.420$ & $6.866 \pm 0.421$ & $5.088 \pm 1.133$ \\
\hline \multicolumn{7}{|l|}{ R. Testis } \\
\hline Absolute & $0.996 \pm 0.035$ & $1.001 \pm 0.034$ & $1.028 \pm 0.022$ & $0.970 \pm 0.036$ & $1.011 \pm 0.031$ & $0.983 \pm 0.047$ \\
\hline Relative & $6.346 \pm 0.134$ & $6.296 \pm 0.096$ & $6.559 \pm 0.269$ & $6.324 \pm 0.128$ & $6.391 \pm 0.136$ & $6.217 \pm 0.180$ \\
\hline \multicolumn{7}{|l|}{ Thymus } \\
\hline Absolute & $0.471 \pm 0.022$ & $0.463 \pm 0.018$ & $0.455 \pm 0.010$ & $0.483 \pm 0.015$ & $0.475 \pm 0.016$ & $0.466 \pm 0.021$ \\
\hline Relative & $2.999 \pm 0.099$ & $2.912 \pm 0.068$ & $2.898 \pm 0.054$ & $3.151 \pm 0.038$ & $3.012 \pm 0.127$ & $2.958 \pm 0.148$ \\
\hline \multicolumn{7}{|l|}{ Female } \\
\hline Necropsy body wt & $120 \pm 2$ & $121 \pm 1$ & $120 \pm 3$ & $122 \pm 4$ & $120 \pm 2$ & $120 \pm 2$ \\
\hline \multicolumn{7}{|l|}{ Heart } \\
\hline Absolute & $0.48 \pm 0.01$ & $0.50 \pm 0.02$ & $0.51 \pm 0.01$ & $0.49 \pm 0.02$ & $0.48 \pm 0.01$ & $0.50 \pm 0.01$ \\
\hline Relative & $4.007 \pm 0.098$ & $4.094 \pm 0.135$ & $4.205 \pm 0.060$ & $4.041 \pm 0.054$ & $4.009 \pm 0.112$ & $4.120 \pm 0.059$ \\
\hline \multicolumn{7}{|l|}{ R. Kidney } \\
\hline Absolute & $0.57 \pm 0.02$ & $0.61 \pm 0.01$ & $0.59 \pm 0.01$ & $0.60 \pm 0.02$ & $0.58 \pm 0.01$ & $0.58 \pm 0.01$ \\
\hline Relative & $4.729 \pm 0.050$ & $4.994 \pm 0.095$ & $4.929 \pm 0.066$ & $4.945 \pm 0.051$ & $4.844 \pm 0.060$ & $4.798 \pm 0.109$ \\
\hline \multicolumn{7}{|l|}{ Liver } \\
\hline Absolute & $5.64 \pm 0.14$ & $5.85 \pm 0.17$ & $5.91 \pm 0.17$ & $6.03 \pm 0.30$ & $5.69 \pm 0.10$ & $6.20 \pm 0.11 b$ \\
\hline Relative & $47.053 \pm 1.335$ & $48.238 \pm 1.369$ & $49.157 \pm 1.602$ & $49.495 \pm 0.914$ & $47.564 \pm 0.707$ & $51.537 \pm 0.476^{b}$ \\
\hline \multicolumn{7}{|l|}{ Lung } \\
\hline Absolute & $0.81 \pm 0.02$ & $0.84 \pm 0.02$ & $0.85 \pm 0.05$ & $0.82 \pm 0.02$ & $0.83 \pm 0.02$ & $0.84 \pm 0.03$ \\
\hline Relative & $6.716 \pm 0.216$ & $6.955 \pm 0.174$ & $7.036 \pm 0.365$ & $6.754 \pm 0.120$ & $6.901 \pm 0.210$ & $6.979 \pm 0.222$ \\
\hline
\end{tabular}


$o$-Chloropyridine, NTP TOX 83

\begin{tabular}{|c|c|c|c|c|c|c|}
\hline & $\begin{array}{l}\text { Vehicle } \\
\text { Control }\end{array}$ & $6.25 \mathrm{mg} / \mathrm{kg}$ & 12.5 mg/kg & $25 \mathrm{mg} / \mathrm{kg}$ & $50 \mathrm{mg} / \mathrm{kg}$ & $100 \mathrm{mg} / \mathrm{kg}$ \\
\hline \multicolumn{7}{|l|}{ Thymus } \\
\hline Absolute & $0.374 \pm 0.012$ & $0.411 \pm 0.025$ & $0.385 \pm 0.011$ & $0.379 \pm 0.027$ & $0.390 \pm 0.008$ & $0.371 \pm 0.018$ \\
\hline Relative & $3.115 \pm 0.104$ & $3.389 \pm 0.212$ & $3.202 \pm 0.090$ & $3.105 \pm 0.122$ & $3.258 \pm 0.076$ & $3.080 \pm 0.097$ \\
\hline
\end{tabular}

*Significantly different $(\mathrm{P} \leq 0.05)$ from the vehicle control group by Williams' test.

$* * \mathrm{P} \leq 0.01$

${ }^{a}$ Organ weights (absolute weights) and body weights are given in grams; organ-weight-to-body-weight ratios (relative weights) are given as $\mathrm{mg}$ organ weight/g body weight (mean \pm standard error).

${ }^{b} \mathrm{n}=4$. 
Table C-2. Organ Weights and Organ-Weight-to-Body-Weight Ratios for Rats in the Three-month Drinking Water Study of $o$-Chloropyridine ${ }^{a}$

\begin{tabular}{|c|c|c|c|c|c|c|}
\hline & O ppm & $10 \mathrm{ppm}$ & $30 \mathrm{ppm}$ & 100 ppm & 300 ppm & $1,000 \mathrm{ppm}$ \\
\hline $\mathbf{n}$ & 10 & 10 & 10 & 10 & 10 & 10 \\
\hline \multicolumn{7}{|l|}{ Male } \\
\hline Necropsy body wt & $320 \pm 6$ & $326 \pm 7$ & $326 \pm 8$ & $326 \pm 8$ & $309 \pm 7$ & $268 \pm 6^{* *}$ \\
\hline \multicolumn{7}{|l|}{ Heart } \\
\hline Absolute & $0.90 \pm 0.02$ & $0.93 \pm 0.03$ & $0.90 \pm 0.03^{b}$ & $0.94 \pm 0.03$ & $0.90 \pm 0.02$ & $0.83 \pm 0.01$ \\
\hline Relative & $2.806 \pm 0.032$ & $2.854 \pm 0.048$ & $2.791 \pm 0.022^{\mathrm{b}}$ & $2.890 \pm 0.079$ & $2.898 \pm 0.032$ & $3.089 \pm 0.046^{* *}$ \\
\hline \multicolumn{7}{|l|}{ R. Kidney } \\
\hline Absolute & $0.95 \pm 0.01$ & $1.02 \pm 0.02 *$ & $1.04 \pm 0.03 *$ & $1.01 \pm 0.02 *$ & $1.02 \pm 0.03 *$ & $1.07 \pm 0.02 * *$ \\
\hline Relative & $2.978 \pm 0.065$ & $3.144 \pm 0.036^{*}$ & $3.172 \pm 0.052^{*}$ & $3.110 \pm 0.081$ & $3.304 \pm 0.030 * *$ & $4.012 \pm 0.052 * *$ \\
\hline \multicolumn{7}{|l|}{ Liver } \\
\hline Absolute & $10.91 \pm 0.29$ & $11.76 \pm 0.29$ & $10.87 \pm 0.78$ & $13.17 \pm 0.35^{* *}$ & $13.51 \pm 0.40 * *$ & $14.33 \pm 0.36^{* *}$ \\
\hline Relative & $34.101 \pm 0.737$ & $36.114 \pm 0.615$ & $33.092 \pm 1.788$ & $40.530 \pm 1.053 * *$ & $43.672 \pm 0.620 * *$ & $53.553 \pm 0.653 * *$ \\
\hline \multicolumn{7}{|l|}{ Lung } \\
\hline Absolute & $1.34 \pm 0.05$ & $1.35 \pm 0.04$ & $1.45 \pm 0.09$ & $1.32 \pm 0.05$ & $1.32 \pm 0.04$ & $1.21 \pm 0.05$ \\
\hline Relative & $4.186 \pm 0.150$ & $4.124 \pm 0.069$ & $4.422 \pm 0.185$ & $4.061 \pm 0.154$ & $4.261 \pm 0.113$ & $4.493 \pm 0.114$ \\
\hline \multicolumn{7}{|l|}{ R. Testis } \\
\hline Absolute & $1.344 \pm 0.030$ & $1.394 \pm 0.029$ & $1.404 \pm 0.021$ & $1.457 \pm 0.028 *$ & $1.421 \pm 0.025$ & $1.423 \pm 0.022$ \\
\hline Relative & $4.212 \pm 0.118$ & $4.282 \pm 0.051$ & $4.323 \pm 0.100$ & $4.490 \pm 0.121$ & $4.611 \pm 0.090 * *$ & $5.330 \pm 0.091 * *$ \\
\hline \multicolumn{7}{|l|}{ Thymus } \\
\hline Absolute & $0.317 \pm 0.014$ & $0.327 \pm 0.009$ & $0.317 \pm 0.010$ & $0.314 \pm 0.011$ & $0.309 \pm 0.014$ & $0.256 \pm 0.008 * *$ \\
\hline Relative & $0.990 \pm 0.040$ & $1.005 \pm 0.032$ & $0.979 \pm 0.043$ & $0.968 \pm 0.039$ & $0.999 \pm 0.038$ & $0.959 \pm 0.030$ \\
\hline
\end{tabular}

\section{Female}

Necropsy body wt

$195 \pm 4$

$200 \pm 2$

$200 \pm 4$

$196 \pm 2$

$190 \pm 3$

$174 \pm 2 * *$

Heart

$\begin{array}{lcccccc}\text { Absolute } & 0.57 \pm 0.01 & 0.58 \pm 0.01 & 0.61 \pm 0.02 & 0.60 \pm 0.02 & 0.59 \pm 0.01 & 0.57 \pm 0.01 \\ \text { Relative } & 2.915 \pm 0.034 & 2.907 \pm 0.035 & 3.043 \pm 0.065 & 3.074 \pm 0.083 & 3.093 \pm 0.064 * & 3.249 \pm 0.031 * *\end{array}$

R. Kidney

Absolute

$0.65 \pm 0.02$

$0.67 \pm 0.01$

$0.70 \pm 0.01 *$

$0.69 \pm 0.02 *$

$0.71 \pm 0.01 * *$

$0.74 \pm 0.01 * *$

Relative

$3.317 \pm 0.047$

$3.347 \pm 0.032$

$3.472 \pm 0.050 *$

$3.530 \pm 0.051 * *$

$3.714 \pm 0.058 * * \quad 4.259 \pm 0.040 * *$

Liver

\begin{tabular}{lcccccc} 
Absolute & $5.80 \pm 0.22$ & $6.14 \pm 0.14$ & $6.77 \pm 0.17 * *$ & $6.89 \pm 0.12 * *$ & $7.94 \pm 0.34 * *$ & $9.06 \pm 0.21 * *$ \\
Relative & $29.632 \pm 0.735$ & $30.698 \pm 0.619$ & $33.784 \pm 0.410^{* *}$ & $35.152 \pm 0.373 * *$ & $41.684 \pm 1.375 * * 2.086 \pm 0.597 * *$ \\
Lung & & & & & \\
Absolute & $0.93 \pm 0.02$ & $0.95 \pm 0.02$ & $0.96 \pm 0.02$ & $0.96 \pm 0.02$ & $0.95 \pm 0.02$ & $0.87 \pm 0.01$ \\
Relative & $4.760 \pm 0.095$ & $4.745 \pm 0.118$ & $4.818 \pm 0.118$ & $4.914 \pm 0.074$ & $5.002 \pm 0.103$ & $5.027 \pm 0.091$ \\
Thymus & & & & & & \\
Absolute & $0.235 \pm 0.008$ & $0.242 \pm 0.009$ & $0.240 \pm 0.006$ & $0.247 \pm 0.016$ & $0.243 \pm 0.010$ & $0.206 \pm 0.006$ \\
Relative & $1.206 \pm 0.037$ & $1.211 \pm 0.044$ & $1.198 \pm 0.031$ & $1.265 \pm 0.087$ & $1.276 \pm 0.046$ & $1.189 \pm 0.037$ \\
\hline
\end{tabular}

*Significantly different $(\mathrm{P} \leq 0.05)$ from the control group by Williams' test.

$* * \mathrm{P} \leq 0.01$.

${ }^{a}$ Organ weights (absolute weights) and body weights are given in grams; organ-weight-to-body-weight ratios (relative weights) are given as $\mathrm{mg}$ organ weight/g body weight (mean \pm standard error).

${ }^{b} \mathrm{n}=9$. 
Table C-3. Organ Weights and Organ-Weight-to-Body-Weight Ratios for Mice in the Two-week Dermal Study of $o$-Chloropyridine ${ }^{\text {a }}$

\begin{tabular}{|c|c|c|c|c|c|c|}
\hline & $\begin{array}{l}\text { Vehicle } \\
\text { Control }\end{array}$ & $6.25 \mathrm{mg} / \mathrm{kg}$ & $12.5 \mathrm{mg} / \mathrm{kg}$ & $25 \mathrm{mg} / \mathrm{kg}$ & $50 \mathrm{mg} / \mathrm{kg}$ & $100 \mathrm{mg} / \mathrm{kg}$ \\
\hline $\mathbf{n}$ & 5 & 5 & 5 & 5 & 5 & 5 \\
\hline \multicolumn{7}{|l|}{ Male } \\
\hline Necropsy body wt & $25.7 \pm 0.3$ & $26.4 \pm 0.5$ & $26.8 \pm 0.4$ & $26.1 \pm 0.5$ & $25.7 \pm 0.3$ & $25.6 \pm 0.7$ \\
\hline \multicolumn{7}{|l|}{ Heart } \\
\hline Absolute & $0.13 \pm 0.01$ & $0.13 \pm 0.00$ & $0.14 \pm 0.00$ & $0.14 \pm 0.01$ & $0.13 \pm 0.00$ & $0.13 \pm 0.01$ \\
\hline Relative & $5.009 \pm 0.181$ & $5.046 \pm 0.070$ & $5.088 \pm 0.079$ & $5.218 \pm 0.136$ & $5.230 \pm 0.069$ & $5.182 \pm 0.129$ \\
\hline \multicolumn{7}{|l|}{ R. Kidney } \\
\hline Absolute & $0.26 \pm 0.00$ & $0.27 \pm 0.01$ & $0.28 \pm 0.00$ & $0.28 \pm 0.01$ & $0.28 \pm 0.01$ & $0.28 \pm 0.01$ \\
\hline Relative & $10.059 \pm 0.254$ & $10.379 \pm 0.134$ & $10.608 \pm 0.230$ & $10.544 \pm 0.316$ & $10.940 \pm 0.277 *$ & $10.921 \pm 0.248^{*}$ \\
\hline \multicolumn{7}{|l|}{ Liver } \\
\hline Absolute & $1.48 \pm 0.02$ & $1.51 \pm 0.02$ & $1.51 \pm 0.03$ & $1.50 \pm 0.04$ & $1.50 \pm 0.01$ & $1.53 \pm 0.04$ \\
\hline Relative & $57.443 \pm 0.881$ & $57.168 \pm 0.753$ & $56.504 \pm 1.101$ & $57.429 \pm 1.473$ & $58.181 \pm 0.698$ & $59.948 \pm 1.673$ \\
\hline \multicolumn{7}{|l|}{ Lung } \\
\hline Absolute & $0.16 \pm 0.01$ & $0.20 \pm 0.02$ & $0.18 \pm 0.02$ & $0.18 \pm 0.01$ & $0.17 \pm 0.01$ & $0.18 \pm 0.01$ \\
\hline Relative & $6.095 \pm 0.472$ & $7.599 \pm 0.625$ & $6.870 \pm 0.831$ & $6.827 \pm 0.514$ & $6.716 \pm 0.303$ & $6.926 \pm 0.305$ \\
\hline \multicolumn{7}{|l|}{ R. Testis } \\
\hline Absolute & $0.110 \pm 0.003$ & $0.105 \pm 0.004$ & $0.106 \pm 0.004$ & $0.108 \pm 0.003$ & $0.113 \pm 0.003$ & $0.103 \pm 0.004$ \\
\hline Relative & $4.265 \pm 0.131$ & $3.974 \pm 0.153$ & $3.979 \pm 0.158$ & $4.130 \pm 0.068$ & $4.390 \pm 0.118$ & $4.042 \pm 0.084$ \\
\hline \multicolumn{7}{|l|}{ Thymus } \\
\hline Absolute & $0.054 \pm 0.003$ & $0.054 \pm 0.001$ & $0.052 \pm 0.003$ & $0.046 \pm 0.001$ & $0.053 \pm 0.002$ & $0.054 \pm 0.005$ \\
\hline Relative & $2.101 \pm 0.104$ & $2.036 \pm 0.040$ & $1.940 \pm 0.090$ & $1.774 \pm 0.067$ & $2.066 \pm 0.096$ & $2.103 \pm 0.150$ \\
\hline \multicolumn{7}{|l|}{ Female } \\
\hline Necropsy body wt & $23.2 \pm 0.6$ & $23.1 \pm 0.4$ & $22.8 \pm 0.6$ & $23.0 \pm 0.6$ & $22.7 \pm 0.6$ & $23.3 \pm 0.4$ \\
\hline \multicolumn{7}{|l|}{ Heart } \\
\hline Absolute & $0.13 \pm 0.01$ & $0.13 \pm 0.01$ & $0.13 \pm 0.01$ & $0.13 \pm 0.00$ & $0.13 \pm 0.00$ & $0.13 \pm 0.00$ \\
\hline Relative & $5.411 \pm 0.164$ & $5.498 \pm 0.130$ & $5.589 \pm 0.091$ & $5.679 \pm 0.052$ & $5.646 \pm 0.140$ & $5.595 \pm 0.099$ \\
\hline \multicolumn{7}{|l|}{ R. Kidney } \\
\hline Absolute & $0.20 \pm 0.01$ & $0.20 \pm 0.01$ & $0.20 \pm 0.00$ & $0.21 \pm 0.01$ & $0.20 \pm 0.01$ & $0.21 \pm 0.01$ \\
\hline Relative & $8.383 \pm 0.185$ & $8.835 \pm 0.119$ & $8.598 \pm 0.185$ & $9.074 \pm 0.133^{*}$ & $8.613 \pm 0.194$ & $8.849 \pm 0.172$ \\
\hline \multicolumn{7}{|l|}{ Liver } \\
\hline Absolute & $1.37 \pm 0.05$ & $1.36 \pm 0.06$ & $1.28 \pm 0.04$ & $1.38 \pm 0.08$ & $1.32 \pm 0.04$ & $1.47 \pm 0.01$ \\
\hline Relative & $58.806 \pm 0.979$ & $58.610 \pm 1.496$ & $56.121 \pm 1.307$ & $60.054 \pm 2.197$ & $58.148 \pm 0.773$ & $63.336 \pm 1.070$ \\
\hline \multicolumn{7}{|l|}{ Lung } \\
\hline Absolute & $0.18 \pm 0.01$ & $0.21 \pm 0.01$ & $0.18 \pm 0.01$ & $0.19 \pm 0.01$ & $0.18 \pm 0.01$ & $0.18 \pm 0.01$ \\
\hline Relative & $7.763 \pm 0.474$ & $8.875 \pm 0.383$ & $8.060 \pm 0.386$ & $8.176 \pm 0.604$ & $7.969 \pm 0.324$ & $7.576 \pm 0.297$ \\
\hline \multicolumn{7}{|l|}{ Thymus } \\
\hline Absolute & $0.075 \pm 0.003$ & $0.070 \pm 0.002$ & $0.066 \pm 0.002$ & $0.071 \pm 0.003$ & $0.079 \pm 0.004$ & $0.077 \pm 0.006$ \\
\hline Relative & $3.231 \pm 0.083$ & $3.056 \pm 0.136$ & $2.900 \pm 0.079$ & $3.107 \pm 0.202$ & $3.475 \pm 0.089$ & $3.316 \pm 0.194$ \\
\hline
\end{tabular}

*Significantly different $(\mathrm{P} \leq 0.05)$ from the vehicle control group by Williams' or Dunnett's test.

${ }^{a}$ Organ weights (absolute weights) and body weights are given in grams; organ-weight-to-body-weight ratios (relative weights) are given as $\mathrm{mg}$ organ weight/g body weight (mean \pm standard error). 
Table C-4. Organ Weights and Organ-Weight-to-Body-Weight Ratios for Mice in the Three-month Drinking Water Study of $o$-Chloropyridine ${ }^{a}$

\begin{tabular}{|c|c|c|c|c|c|c|}
\hline & O ppm & 10 ppm & 30 ppm & 100 ppm & 300 ppm & 1,000 ppm \\
\hline \multicolumn{7}{|l|}{ Male } \\
\hline $\mathbf{n}$ & 10 & 10 & 10 & 10 & 10 & 10 \\
\hline $\begin{array}{l}\text { Necropsy body } \\
\text { wt }\end{array}$ & $40.8 \pm 0.9$ & $42.4 \pm 1.1$ & $42.4 \pm 0.6$ & $40.2 \pm 1.2$ & $40.5 \pm 1.2$ & $33.0 \pm 0.5^{* *}$ \\
\hline \multicolumn{7}{|l|}{ Heart } \\
\hline Absolute & $0.17 \pm 0.00$ & $0.17 \pm 0.00$ & $0.18 \pm 0.00$ & $0.17 \pm 0.00$ & $0.17 \pm 0.00$ & $0.15 \pm 0.00 * *$ \\
\hline Relative & $4.116 \pm 0.070$ & $4.108 \pm 0.112$ & $4.293 \pm 0.089$ & $4.169 \pm 0.091$ & $4.129 \pm 0.105$ & $4.573 \pm 0.059 * *$ \\
\hline \multicolumn{7}{|l|}{ R. Kidney } \\
\hline Absolute & $0.34 \pm 0.01$ & $0.35 \pm 0.01$ & $0.35 \pm 0.01$ & $0.35 \pm 0.01$ & $0.35 \pm 0.01$ & $0.31 \pm 0.01$ \\
\hline Relative & $8.237 \pm 0.218$ & $8.203 \pm 0.090$ & $8.193 \pm 0.244$ & $8.631 \pm 0.217$ & $8.556 \pm 0.229$ & $9.242 \pm 0.369 *$ \\
\hline \multicolumn{7}{|l|}{ Liver } \\
\hline Absolute & $1.70 \pm 0.06$ & $1.89 \pm 0.07 *$ & $1.95 \pm 0.04^{*}$ & $1.84 \pm 0.07 *$ & $2.01 \pm 0.08 * *$ & $1.95 \pm 0.05 * *$ \\
\hline Relative & $41.624 \pm 0.778$ & $44.531 \pm 0.718^{*}$ & $45.851 \pm 0.819^{* *}$ & $45.766 \pm 0.974 * *$ & $49.467 \pm 0.796^{* *}$ & $59.121 \pm 0.830^{* *}$ \\
\hline \multicolumn{7}{|l|}{ Lung } \\
\hline Absolute & $0.28 \pm 0.02$ & $0.27 \pm 0.02$ & $0.26 \pm 0.02$ & $0.27 \pm 0.02$ & $0.29 \pm 0.02$ & $0.29 \pm 0.01$ \\
\hline Relative & $6.824 \pm 0.375$ & $6.479 \pm 0.445$ & $6.230 \pm 0.489$ & $6.806 \pm 0.423$ & $7.248 \pm 0.507$ & $8.676 \pm 0.441 * *$ \\
\hline \multicolumn{7}{|l|}{ R. Testis } \\
\hline Absolute & $0.119 \pm 0.001$ & $0.119 \pm 0.002$ & $0.121 \pm 0.002$ & $0.117 \pm 0.002$ & $0.120 \pm 0.003$ & $0.116 \pm 0.004$ \\
\hline Relative & $2.926 \pm 0.047$ & $2.810 \pm 0.067$ & $2.857 \pm 0.046$ & $2.945 \pm 0.102$ & $2.969 \pm 0.079$ & $3.502 \pm 0.093 * *$ \\
\hline \multicolumn{7}{|l|}{ Thymus } \\
\hline Absolute & $0.054 \pm 0.004$ & $0.061 \pm 0.006^{\mathrm{b}}$ & $0.054 \pm 0.002$ & $0.052 \pm 0.002$ & $0.057 \pm 0.004$ & $0.043 \pm 0.002$ \\
\hline Relative & $1.328 \pm 0.075$ & $1.428 \pm 0.123^{\mathrm{b}}$ & $1.264 \pm 0.054$ & $1.293 \pm 0.047$ & $1.401 \pm 0.097$ & $1.308 \pm 0.069$ \\
\hline
\end{tabular}

Female

\begin{tabular}{lcccccc}
$\mathbf{n}$ & 9 & 10 & 10 & 10 & 10 & 10 \\
\hline $\begin{array}{l}\text { Necropsy body } \\
\text { wt }\end{array}$ & $28.5 \pm 1.4$ & $31.8 \pm 1.0$ & $31.2 \pm 1.3$ & $30.3 \pm 1.1$ & $31.9 \pm 0.8$ & $28.4 \pm 0.6$
\end{tabular}

Heart

\begin{tabular}{ccccccc} 
Absolute & $0.13 \pm 0.01$ & $0.13 \pm 0.00$ & $0.13 \pm 0.00$ & $0.12 \pm 0.00$ & $0.13 \pm 0.00$ & $0.13 \pm 0.00$ \\
Relative & $4.511 \pm 0.153$ & $4.038 \pm 0.143^{*}$ & $4.181 \pm 0.134$ & $4.130 \pm 0.131$ & $4.102 \pm 0.092$ & $4.494 \pm 0.063$ \\
R. Kidney & & & & & & \\
Absolute & $0.19 \pm 0.01$ & $0.20 \pm 0.00$ & $0.19 \pm 0.01$ & $0.19 \pm 0.01$ & $0.20 \pm 0.00$ & $0.22 \pm 0.01^{* *}$ \\
Relative & $6.866 \pm 0.352$ & $6.203 \pm 0.206$ & $6.212 \pm 0.170$ & $6.232 \pm 0.174$ & $6.321 \pm 0.154$ & $7.676 \pm 0.156^{*}$ \\
Liver & & & & & & \\
Absolute & $1.22 \pm 0.09$ & $1.37 \pm 0.04$ & $1.34 \pm 0.05$ & $1.35 \pm 0.07$ & $1.62 \pm 0.05^{* *}$ & $1.94 \pm 0.0 .05^{* *}$ \\
Relative & $42.778 \pm 1.560$ & $43.065 \pm 1.066$ & $43.266 \pm 0.645$ & $44.466 \pm 1.122$ & $50.818 \pm 1.290^{* *}$ & $68.154 \pm 1.026^{* *}$ \\
\hline
\end{tabular}


$o$-Chloropyridine, NTP TOX 83

\begin{tabular}{|c|c|c|c|c|c|c|}
\hline & O ppm & 10 ppm & 30 ppm & 100 ppm & 300 ppm & 1,000 ppm \\
\hline \multicolumn{7}{|l|}{ Lung } \\
\hline Absolute & $0.25 \pm 0.02$ & $0.27 \pm 0.02$ & $0.25 \pm 0.02$ & $0.23 \pm 0.01$ & $0.24 \pm 0.01$ & $0.23 \pm 0.01$ \\
\hline Relative & $8.952 \pm 0.699$ & $8.502 \pm 0.795$ & $7.981 \pm 0.537$ & $7.523 \pm 0.440$ & $7.535 \pm 0.324$ & $7.987 \pm 0.380$ \\
\hline \multicolumn{7}{|l|}{ Thymus } \\
\hline Absolute & $0.049 \pm 0.004$ & $0.056 \pm 0.003$ & $0.054 \pm 0.003$ & $0.054 \pm 0.002$ & $0.053 \pm 0.002$ & $0.045 \pm 0.002$ \\
\hline Relative & $1.715 \pm 0.111$ & $1.762 \pm 0.123$ & $1.722 \pm 0.077$ & $1.812 \pm 0.073$ & $1.679 \pm 0.072$ & $1.578 \pm 0.077$ \\
\hline
\end{tabular}

*Significantly different $(\mathrm{P} \leq 0.05)$ from the control group by Williams' or Dunnett's test. $* * \mathrm{P} \leq 0.01$.

${ }^{\mathrm{a}}$ Organ weights (absolute weights) and body weights are given in grams; organ-weight-to-body-weight ratios (relative weights) are given as $\mathrm{mg}$ organ weight/g body weight (mean \pm standard error).

$\mathrm{b}_{\mathrm{n}}=9$. 


\section{Appendix D. Reproductive Tissue Evaluations and Estrous Cycle Characterization}

\section{Tables}

Table D-1. Summary of Reproductive Tissue Evaluations for Male Rats in the Three-month Drinking Water Study of $o$-Chloropyridine.

Table D-2. Estrous Cycle Characterization for Female Rats in the Three-month Drinking Water Study of $o$-Chloropyridine

Table D-3. Results of Vaginal Cytology Study Using the Transition Matrix Approach for Female Rats in the Three-month Drinking Water Study of $o$-Chloropyridine

Table D-4. Summary of Reproductive Tissue Evaluations for Male Mice in the Three-month Drinking Water Study of $o$-Chloropyridine.

Table D-5. Estrous Cycle Characterization for Female Mice in the Three-month Drinking Water Study of $o$-Chloropyridine

Table D-6. Results of Vaginal Cytology Study Using the Transition Matrix Approach for Female Mice in the Three-month Drinking Water Study of $o$-Chloropyridine D-5

\section{Figures}

Figure D-1. Vaginal Cytology Plots for Female Rats in the Three-month Drinking Water Study of $o$-Chloropyridine D-6

Figure D-2. Vaginal Cytology Plots for Female Mice in the Three-month Drinking Water Study of $o$-Chloropyridine 
Table D-1. Summary of Reproductive Tissue Evaluations for Male Rats in the Three-month Drinking Water Study of $o$-Chloropyridine ${ }^{a}$

\begin{tabular}{lcccc}
\hline & $\mathbf{0 ~} \mathbf{~ p m}$ & $\mathbf{1 0 0} \mathbf{~ p p m}$ & $\mathbf{3 0 0} \mathbf{~ p p m}$ & $\mathbf{1 , 0 0 0} \mathbf{~ p p m}$ \\
\hline $\mathbf{n}$ & 10 & 10 & 10 & 10 \\
\hline Weights (g) & & & & \\
$\quad$ Necropsy body wt & $320 \pm 6$ & $326 \pm 8$ & $309 \pm 7$ & $268 \pm 6^{* *}$ \\
$\quad$ L. Cauda epididymis & $0.1510 \pm 0.0067$ & $0.1563 \pm 0.0040$ & $0.1548 \pm 0.0041$ & $0.1503 \pm 0.0037$ \\
L. Epididymis & $0.4239 \pm 0.0177$ & $0.4579 \pm 0.0097$ & $0.4457 \pm 0.0063$ & $0.4222 \pm 0.0079$ \\
L. Testis & $1.4547 \pm 0.0387$ & $1.5359 \pm 0.0227$ & $1.4795 \pm 0.0269$ & $1.4671 \pm 0.0212$ \\
Spermatid measurements & & & & \\
$\quad$ Spermatid heads (10\%/testis) & $169.75 \pm 7.20$ & $182.13 \pm 5.88$ & $170.88 \pm 4.14$ & $166.75 \pm 4.25$ \\
$\quad$ Spermatid heads (10\%/g testis) & $129.7 \pm 3.6$ & $131.9 \pm 4.6$ & $126.8 \pm 2.8$ & $125.6 \pm 3.0$ \\
Epididymal spermatozoal measurements & & & \\
$\quad$ Sperm motility (\%) & $77.6 \pm 1.0$ & $71.7 \pm 8.0$ & $76.9 \pm 1.4$ & $77.4 \pm 1.0$ \\
Sperm (10\%/cauda epididymis) & $60.00 \pm 4.43$ & $68.75 \pm 6.84$ & $62.15 \pm 5.53$ & $42.40 \pm 2.02^{*}$ \\
$\quad$ Sperm (10\%/g cauda epididymis) & $402 \pm 29$ & $443 \pm 46$ & $400 \pm 32$ & $283 \pm 13^{*}$ \\
\hline
\end{tabular}

*Significantly different $(\mathrm{P} \leq 0.05)$ from the control group by Shirley's or Dunn's test.

**Significantly different $(\mathrm{P} \leq 0.01)$ from the control group by Williams' test.

aData are presented as mean \pm standard error. Differences from the control group are not significant by Dunnett's test (tissue weights) or Dunn's test (spermatid measurements and sperm motility).

Table D-2. Estrous Cycle Characterization for Female Rats in the Three-month Drinking Water Study of $o$-Chloropyridine ${ }^{\text {a }}$

\begin{tabular}{lcccc}
\hline & $\mathbf{0 ~} \mathbf{~ p m}$ & $\mathbf{1 0 0} \mathbf{~ p p m}$ & $\mathbf{3 0 0} \mathbf{~ p p m}$ & $\mathbf{1 , 0 0 0} \mathbf{~ p p m}$ \\
\hline Number weighed at necropsy & 10 & 10 & 10 & 10 \\
$\quad$ Necropsy body wt (g) & $195 \pm 4$ & $196 \pm 2$ & $190 \pm 3$ & $174 \pm 2^{* *}$ \\
Proportion of regular cycling females ${ }^{\mathrm{b}}$ & $9 / 10$ & $10 / 10$ & $8 / 10$ & $9 / 10$ \\
Estrous cycle length (days) & $5.50 \pm 0.27$ & $5.00 \pm 0.15$ & $5.50 \pm 0.24^{\mathrm{c}}$ & $5.90 \pm 0.18$ \\
Estrous stages (\% of cycle) & & & & \\
Diestrus & 53.3 & 54.2 & 62.5 & 61.7 \\
Proestrus & 15.0 & 14.2 & 11.7 & 13.3 \\
Estrus & 23.3 & 20.8 & 20.8 & 19.2 \\
Metestrus & 8.3 & 10.8 & 5.0 & 5.8 \\
\hline
\end{tabular}

**Significantly different $(\mathrm{P} \leq 0.01)$ from the control group by Williams' test.

${ }^{a}$ Necropsy body weights and estrous cycle length data are presented as mean \pm standard error. Differences from the control group are not significant by Dunn's test (estrous cycle length). By multivariate analysis of variance, exposed females do not differ significantly from the control females in the relative length of time spent in the estrous stages. Tests for equality of transition probability matrices among all groups and between the control group and each exposed group indicated 300 ppm females had a higher proportion of extended diestrus than control females.

${ }^{b}$ Number of females with a regular cycle/number of females cycling.

'Estrous cycle was longer than 12 days or unclear in 1 of 10 animals. 
Table D-3. Results of Vaginal Cytology Study Using the Transition Matrix Approach for Female Rats in the Three-month Drinking Water Study of $o$-Chloropyridine

\begin{tabular}{|c|c|c|c|}
\hline Stage & Comparison & P Value & Trend $^{\mathrm{a}}$ \\
\hline Overall tests & Overall & 0.024 & \\
\hline Overall tests & 100 ppm vs. controls & 0.257 & $\mathrm{~N}$ \\
\hline Overall tests & 300 ppm vs. controls & 0.009 & - \\
\hline Overall tests & 1,000 vs. controls & 0.32 & - \\
\hline Extended estrus & Overall & 0.721 & \\
\hline Extended estrus & 100 ppm vs. controls & 0.305 & $\mathrm{~N}$ \\
\hline Extended estrus & 300 ppm vs. controls & 0.595 & $\mathrm{~N}$ \\
\hline Extended estrus & 1,000 vs. controls & 0.878 & $\mathrm{~N}$ \\
\hline Extended diestrus & Overall & 0.039 & \\
\hline Extended diestrus & 100 ppm vs. controls & 0.695 & $\mathrm{~N}$ \\
\hline Extended diestrus & 300 ppm vs. controls & 0.008 & - \\
\hline Extended diestrus & 1,000 vs. controls & 0.249 & - \\
\hline Extended metestrus & Overall & 1 & \\
\hline Extended metestrus & 100 ppm vs. controls & 1 & - \\
\hline Extended metestrus & 300 ppm vs. controls & 1 & - \\
\hline Extended metestrus & 1,000 vs. controls & 1 & - \\
\hline Extended proestrus & Overall & 1 & \\
\hline Extended proestrus & 100 ppm vs. controls & 1 & - \\
\hline Extended proestrus & 300 ppm vs. controls & 1 & - \\
\hline Extended Proestrus & 1,000 vs. controls & 1 & - \\
\hline Skipped estrus & Overall & 1 & \\
\hline Skipped estrus & 100 ppm vs. controls & 1 & - \\
\hline Skipped estrus & 300 ppm vs. controls & 1 & - \\
\hline Skipped estrus & 1,000 vs. controls & 1 & - \\
\hline Skipped diestrus & Overall & 1 & \\
\hline Skipped diestrus & 100 ppm vs. controls & 1 & - \\
\hline Skipped diestrus & 300 ppm vs. controls & 1 & - \\
\hline Skipped diestrus & 1,000 vs. controls & 1 & - \\
\hline Overall tests & 300 ppm vs. controls & 0.009 & - \\
\hline Extended diestrus & 300 ppm vs. controls & 0.008 & - \\
\hline
\end{tabular}

${ }^{\mathrm{a}} \mathrm{N}$ means that the exposed group had a lower probability of transitioning to and/or from the relevant abnormal state (extended estrus, extended metestrus, extended proestrus, skipped estrus, or skipped diestrus) than did the control group. 
Table D-4. Summary of Reproductive Tissue Evaluations for Male Mice in the Three-month Drinking Water Study of $o$-Chloropyridine ${ }^{a}$

\begin{tabular}{lcccc}
\hline & $\mathbf{0 ~} \mathbf{~ p m}$ & $\mathbf{1 0 0} \mathbf{~ p p m}$ & $\mathbf{3 0 0} \mathbf{~ p p m}$ & $\mathbf{1 , 0 0 0} \mathbf{~ p p m}$ \\
\hline $\mathbf{n}$ & 10 & 10 & 10 & 10 \\
\hline Weights (g) & & & & \\
$\quad$ Necropsy body wt & $40.8 \pm 0.9$ & $40.2 \pm 1.2$ & $40.5 \pm 1.2$ & $33.0 \pm 0.5^{* *}$ \\
$\quad$ L. Cauda epididymis & $0.0200 \pm 0.0010$ & $0.0194 \pm 0.0012$ & $0.0173 \pm 0.0008$ & $0.0201 \pm 0.0009$ \\
L. Epididymis & $0.0509 \pm 0.0019$ & $0.0545 \pm 0.0017$ & $0.0548 \pm 0.0019$ & $0.0530 \pm 0.0020$ \\
L. Testis & $0.1133 \pm 0.0020$ & $0.1132 \pm 0.0012$ & $0.1158 \pm 0.0022$ & $0.1101 \pm 0.0022$ \\
Spermatid measurements & & & & \\
$\quad$ Spermatid heads (10\%/testis) & $19.87 \pm 0.88$ & $19.39 \pm 0.91$ & $20.32 \pm 0.60$ & $19.84 \pm 0.70$ \\
$\quad$ Spermatid heads (10\%/g testis) & $195.3 \pm 9.0$ & $186.4 \pm 8.0$ & $190.1 \pm 2.2$ & $194.8 \pm 5.2$ \\
Epididymal spermatozoal measurements & & & & \\
$\quad$ Sperm motility (\%) & $74.1 \pm 1.3$ & $72.8 \pm 3.7$ & $72.0 \pm 1.7$ & $69.6 \pm 2.7$ \\
Sperm (10\%/cauda epididymis) & $8.99 \pm 1.25$ & $10.29 \pm 0.95$ & $6.92 \pm 0.80$ & $8.99 \pm 1.36$ \\
$\quad$ Sperm (10\%/g cauda epididymis) & $460 \pm 68$ & $554 \pm 64$ & $403 \pm 43$ & $444 \pm 61$ \\
\hline
\end{tabular}

**Significantly different $(\mathrm{P} \leq 0.01)$ from the control group by Williams' test.

aData are presented as mean \pm standard error. Differences from the control group are not significant by Dunnett's test (tissue weights) or Dunn's test (spermatid and epididymal spermatozoal measurements).

Table D-5. Estrous Cycle Characterization for Female Mice in the Three-month Drinking Water Study of $o$-Chloropyridine ${ }^{\text {a }}$

\begin{tabular}{lcccc}
\hline & $\mathbf{0 ~} \mathbf{~ p m}$ & $\mathbf{1 0 0} \mathbf{~ p p m}$ & $\mathbf{3 0 0} \mathbf{~ p p m}$ & $\mathbf{1 , 0 0 0} \mathbf{~ p p m}$ \\
\hline Number weighed at necropsy & 9 & 10 & 10 & 10 \\
$\quad$ Necropsy body wt (g) & $28.5 \pm 1.4$ & $30.3 \pm 1.1$ & $31.9 \pm 0.8$ & $28.4 \pm 0.6$ \\
Proportion of regular cycling females $^{\mathrm{b}}$ & $6 / 9$ & $6 / 10$ & $8 / 10$ & $6 / 10$ \\
Estrous cycle length (days) & $3.86 \pm 0.14^{\mathrm{c}}$ & $4.45 \pm 0.17$ & $3.91 \pm 0.14$ & $5.05 \pm 0.59$ \\
Estrous stages (\% of cycle) & & & & \\
Diestrus & 42.6 & 30.8 & 32.5 & 36.7 \\
Proestrus & 0.0 & 0.0 & 0.0 & 0.0 \\
Estrus & 39.8 & 47.5 & 46.7 & 42.5 \\
Metestrus & 17.6 & 21.7 & 20.8 & 20.8
\end{tabular}

${ }^{a}$ Necropsy body weights and estrous cycle length data are presented as mean \pm standard error. Differences from the control group are not significant by Dunnett's test (body weight) or Dunn's test (estrous cycle length). By multivariate analysis of variance, exposed females do not differ significantly from the control females in the relative length of time spent in the estrous stages. Tests for equality of transition probability matrices among all groups and between the control group and each exposed group indicated exposed females did not have significantly higher proportions of extended estrus or diestrus than control females.

${ }^{b}$ Number of females with a regular cycle/number of females cycling.

'Estrous cycle was longer than 12 days or unclear in 2 of 9 animals. 
Table D-6. Results of Vaginal Cytology Study Using the Transition Matrix Approach for Female Mice in the Three-month Drinking Water Study of $o$-Chloropyridine

\begin{tabular}{|c|c|c|c|}
\hline Stage & Comparison & P Value & Trend $^{\mathrm{a}}$ \\
\hline Overall tests & Overall & $<0.001$ & \\
\hline Overall tests & 100 ppm vs. controls & $<0.001$ & - \\
\hline Overall tests & 300 ppm vs. controls & 0.088 & $\mathrm{~N}$ \\
\hline Overall tests & 1,000 vs. controls & 0.048 & - \\
\hline Extended estrus & Overall & 0.236 & \\
\hline Extended estrus & 100 ppm vs. controls & 0.234 & - \\
\hline Extended estrus & 300 ppm vs. controls & 0.361 & - \\
\hline Extended estrus & 1,000 vs. controls & 0.214 & - \\
\hline Extended diestrus & Overall & 0.074 & \\
\hline Extended diestrus & 100 ppm vs. controls & 0.033 & $\mathrm{~N}$ \\
\hline Extended diestrus & 300 ppm vs. controls & 0.131 & $\mathrm{~N}$ \\
\hline Extended diestrus & 1,000 vs. controls & 0.748 & $\mathrm{~N}$ \\
\hline Extended metestrus & Overall & 1 & \\
\hline Extended metestrus & 100 ppm vs. controls & 1 & - \\
\hline Extended metestrus & 300 ppm vs. controls & 1 & - \\
\hline Extended metestrus & 1,000 vs. controls & 1 & - \\
\hline Extended proestrus & Overall & 1 & \\
\hline Extended proestrus & 100 ppm vs. controls & 1 & - \\
\hline Extended proestrus & 300 ppm vs. controls & 1 & - \\
\hline Extended Proestrus & 1,000 vs. controls & 1 & - \\
\hline Skipped estrus & Overall & 1 & \\
\hline Skipped estrus & 100 ppm vs. controls & 1 & - \\
\hline Skipped estrus & 300 ppm vs. controls & 1 & - \\
\hline Skipped estrus & 1,000 vs. controls & 1 & - \\
\hline Skipped diestrus & Overall & 0.521 & \\
\hline Skipped diestrus & 100 ppm vs. controls & 0.289 & $\mathrm{~N}$ \\
\hline Skipped diestrus & 300 ppm vs. controls & 0.289 & $\mathrm{~N}$ \\
\hline Skipped diestrus & 1,000 vs. controls & 0.916 & $\mathrm{~N}$ \\
\hline Overall tests & 100 ppm vs. controls & $<0.001$ & - \\
\hline Overall tests & 1,000 ppm vs. controls & 0.048 & - \\
\hline Extended diestrus & 100 ppm vs. controls & 0.033 & $\mathrm{~N}$ \\
\hline
\end{tabular}

${ }^{\mathrm{a} N}$ means that the exposed group had a lower probability of transitioning to and/or from the relevant abnormal state (extended estrus, extended metestrus, extended proestrus, skipped estrus, or skipped diestrus) than did the control group. 


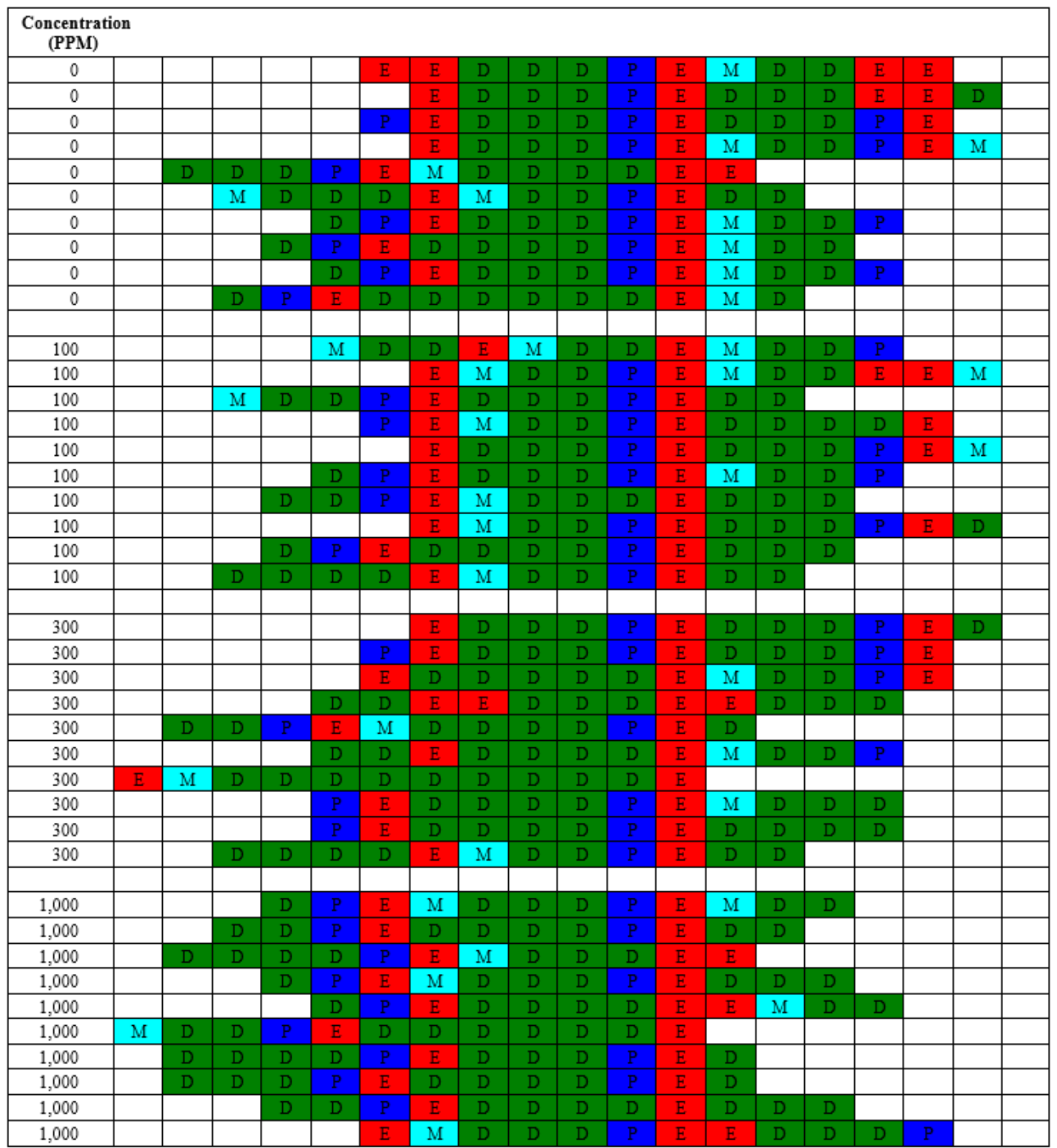

Figure D-1. Vaginal Cytology Plots for Female Rats in the Three-month Drinking Water Study of $o$-Chloropyridine

Daily vaginal lavage samples were collected from each animal and estrous stage determined based on vaginal cytology. Individual females are aligned by their second estrus, and color coded based on estrous stage to aid in visual comparisons amongst the groups. $\mathrm{D}=$ diestrus, $\mathrm{P}=$ proestrus, $\mathrm{E}=$ estrus, $\mathrm{M}=$ metestrus. 


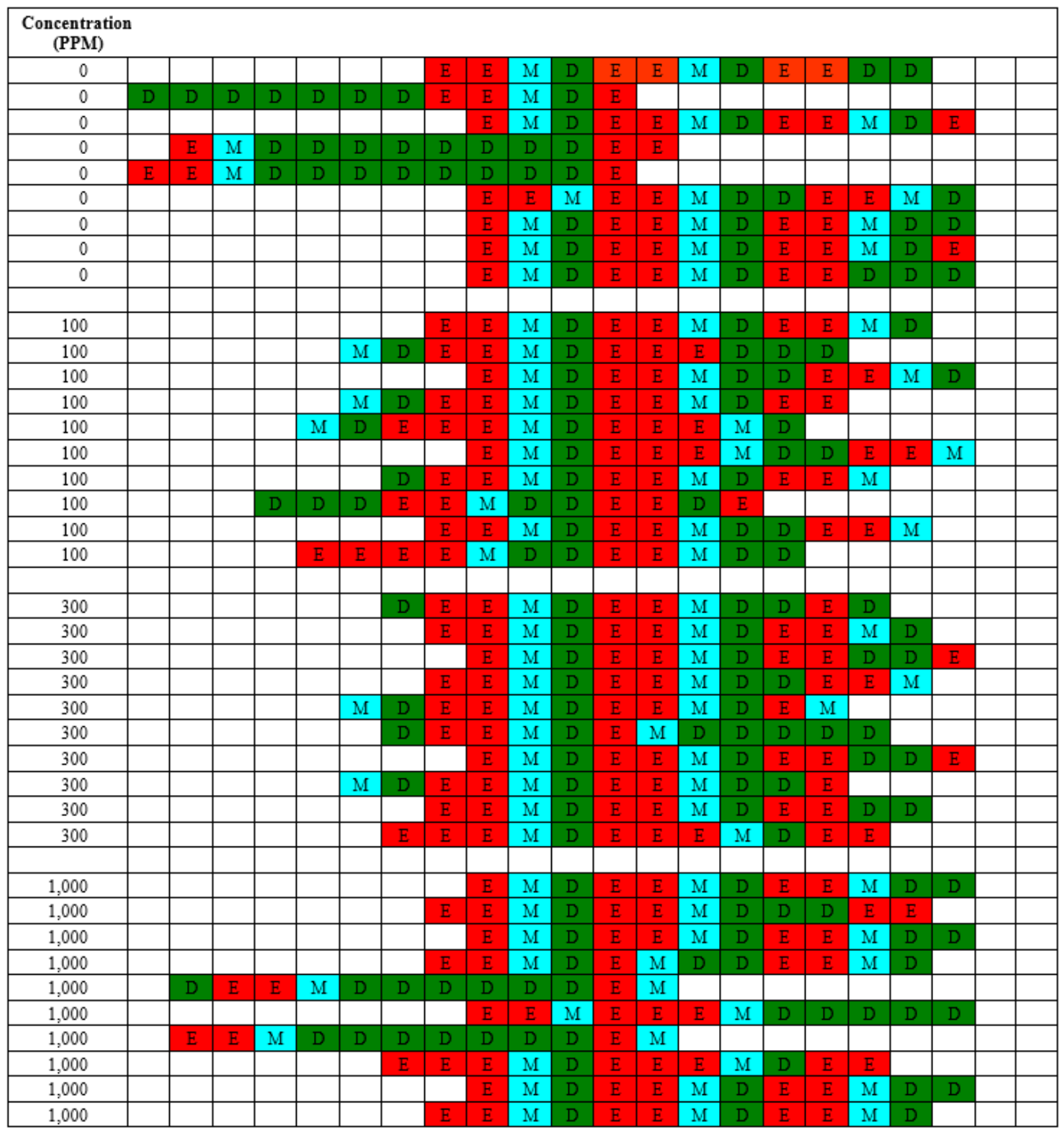

Figure D-2. Vaginal Cytology Plots for Female Mice in the Three-month Drinking Water Study of $o$-Chloropyridine

Daily vaginal lavage samples were collected from each animal and estrous stage determined based on vaginal cytology. Individual females are aligned by their second estrus, and color coded based on estrous stage to aid in visual comparisons amongst the groups. $\mathrm{D}=$ diestrus, $\mathrm{P}=$ proestrus, $\mathrm{E}=$ estrus, $\mathrm{M}=$ metestrus. 


\section{Appendix E. Genetic Toxicology}

\section{Tables}

Table E-1. Mutagenicity of $o$-Chloropyridine in Salmonella typhimurium.

E-2

Table E-2. Frequency of Micronuclei in Peripheral Blood Erythrocytes of Mice Following Administration of $o$-Chloropyridine in Drinking Water for Three Months. 
$o$-Chloropyridine, NTP TOX 83

Table E-1. Mutagenicity of $o$-Chloropyridine in Salmonella typhimurium ${ }^{\text {a }}$

\begin{tabular}{|c|c|c|c|c|c|c|}
\hline Strain & $\begin{array}{c}\text { Dose } \\
\text { ( } \mu \mathrm{g} / \text { plate })\end{array}$ & Without S9 & $\begin{array}{c}\text { With } 30 \% \\
\text { Hamster S9 }\end{array}$ & $\begin{array}{c}\text { With } 30 \% \\
\text { Hamster S9 }\end{array}$ & $\begin{array}{c}\text { With 30\% } \\
\text { Rat S9 }\end{array}$ & $\begin{array}{c}\text { With 30\% } \\
\text { Rat S9 }\end{array}$ \\
\hline \multirow[t]{12}{*}{ TA100 } & 0 & $116 \pm 6$ & $111 \pm 9$ & $127 \pm 6$ & $151 \pm 4$ & $128 \pm 6$ \\
\hline & 10 & & & $127 \pm 1$ & & \\
\hline & 33 & & & $155 \pm 13$ & & \\
\hline & 100 & $130 \pm 2$ & $220 \pm 8$ & $231 \pm 18$ & $153 \pm 7$ & \\
\hline & 166 & & & $362 \pm 13$ & & \\
\hline & 333 & $137 \pm 4$ & $461 \pm 32$ & $496 \pm 18$ & $168 \pm 10$ & $159 \pm 6$ \\
\hline & 666 & & & & & $180 \pm 8$ \\
\hline & 1,000 & $114 \pm 9$ & $1,254 \pm 8$ & & $294 \pm 4$ & $251 \pm 19$ \\
\hline & 1,666 & & & & & $358 \pm 17$ \\
\hline & 3,333 & $126 \pm 5$ & $1,146 \pm 24$ & & $557 \pm 21$ & $554 \pm 20$ \\
\hline & 6,666 & $117 \pm 1$ & & & & \\
\hline & 10,000 & & Toxic & & $157 \pm 14^{\mathrm{b}}$ & \\
\hline Trial summary & & Negative & Positive & Positive & Positive & Positive \\
\hline Positive control $^{c}$ & & $850 \pm 20$ & $645 \pm 18$ & $661 \pm 19$ & $541 \pm 19$ & $626 \pm 13$ \\
\hline \multirow[t]{10}{*}{ TA98 } & 0 & $22 \pm 2$ & $21 \pm 2$ & $24 \pm 3$ & $16 \pm 3$ & $22 \pm 2$ \\
\hline & 100 & $20 \pm 1$ & $27 \pm 1$ & $30 \pm 1$ & $21 \pm 4$ & \\
\hline & 166 & & & $45 \pm 2$ & & \\
\hline & 333 & $19 \pm 3$ & $57 \pm 7$ & $71 \pm 2$ & $19 \pm 3$ & $25 \pm 2$ \\
\hline & 666 & & & $106 \pm 4$ & & $34 \pm 3$ \\
\hline & 1,000 & $22 \pm 1$ & $256 \pm 6$ & $251 \pm 20$ & $43 \pm 3$ & $48 \pm 2$ \\
\hline & 1,666 & & & & & $52 \pm 1$ \\
\hline & 3,333 & $21 \pm 4$ & $286 \pm 4$ & & $68 \pm 3$ & $64 \pm 3$ \\
\hline & 6,666 & $17 \pm 1$ & & & & \\
\hline & 10,000 & & Toxic & & $6 \pm 1^{\mathrm{d}}$ & \\
\hline Trial summary & & Negative & Positive & Positive & Positive & Positive \\
\hline Positive control & & $421 \pm 28$ & $311 \pm 6$ & $457 \pm 15$ & $251 \pm 13$ & $410 \pm 6$ \\
\hline
\end{tabular}


Table E-2. Frequency of Micronuclei in Peripheral Blood Erythrocytes of Mice Following Administration of $o$-Chloropyridine in Drinking Water for Three Months ${ }^{\text {a }}$

\begin{tabular}{|c|c|c|c|c|c|}
\hline & $\begin{array}{c}\text { Exposure } \\
\text { Concentration } \\
(\text { ppm })\end{array}$ & $\begin{array}{l}\text { Number of Mice } \\
\text { with Erythrocytes } \\
\text { Scored }\end{array}$ & $\begin{array}{l}\text { Micronucleated } \\
\text { NCEs/1,000 NCEs }\end{array}$ & P Value ${ }^{c}$ & $\operatorname{PCEs}^{\mathrm{b}}(\%)$ \\
\hline \multicolumn{6}{|l|}{ Male } \\
\hline Water $^{\mathrm{d}}$ & 0 & 5 & $4.00 \pm 0.35$ & & $3.92 \pm 0.35$ \\
\hline \multirow[t]{6}{*}{$o$-Chloropyridine } & 10 & 5 & $3.90 \pm 0.60$ & 0.5449 & $4.34 \pm 0.55$ \\
\hline & 30 & 5 & $3.80 \pm 0.37$ & 0.5897 & $3.66 \pm 0.46$ \\
\hline & 100 & 5 & $3.10 \pm 0.33$ & 0.8577 & $3.20 \pm 0.28$ \\
\hline & 300 & 5 & $2.70 \pm 0.41$ & 0.9442 & $3.56 \pm 0.19$ \\
\hline & 1,000 & 5 & $2.70 \pm 0.20$ & 0.9442 & $3.80 \pm 0.22$ \\
\hline & & & $\mathrm{P}=0.959^{\mathrm{e}}$ & & \\
\hline \multicolumn{6}{|l|}{ Female } \\
\hline Water & 0 & 5 & $2.40 \pm 0.24$ & & $3.52 \pm 0.57$ \\
\hline \multirow[t]{6}{*}{$o$-Chloropyridine } & 10 & 5 & $1.60 \pm 0.29$ & 0.8973 & $3.72 \pm 0.23$ \\
\hline & 30 & 5 & $2.90 \pm 0.64$ & 0.2458 & $4.54 \pm 0.39$ \\
\hline & 100 & 5 & $1.50 \pm 0.32$ & 0.9254 & $3.94 \pm 0.53$ \\
\hline & 300 & 5 & $2.60 \pm 0.29$ & 0.3885 & $3.88 \pm 0.56$ \\
\hline & 1,000 & 5 & $2.10 \pm 0.48$ & 0.6728 & $3.50 \pm 0.24$ \\
\hline & & & $P=0.511$ & & \\
\hline
\end{tabular}

aStudy was performed at ILS, Inc. The detailed protocol is presented by MacGregor et al. ${ }^{48}$. NCE $=$ normochromatic erythrocyte; $\mathrm{PCE}=$ polychromatic erythrocyte.

${ }^{b}$ Mean \pm standard error.

'Pairwise comparison with the untreated control group; exposed group values are significant at $\mathrm{P} \leq 0.005$.

${ }^{\mathrm{d}}$ Untreated control.

${ }^{\mathrm{e}}$ Significance of micronucleated NCEs/1,000 NCEs tested by the one-tailed trend test; significant at $\mathrm{P} \leq 0.025$. 


\section{Appendix F. Chemical Characterization and Dose Formulation Studies}

\section{Table of Contents}

F.1. Procurement and Characterization.

F.2. Preparation and Analysis of Dose Formulations

\section{Tables}

Table F-1. Gas Chromatography Systems Used in the Studies of $o$-Chloropyridine F-4

Table F-2. Preparation and Storage of Dose Formulations in the Studies of $o$-Chloropyridine

Table F-3. Results of Analyses of Dose Formulations Administered to Rats and Mice in the Two-week Dermal Studies of $o$-Chloropyridine

Table F-4. Results of Analyses of Dose Formulations Administered to Rats and Mice in the Three-month Drinking Water Studies of $o$-Chloropyridine

\section{Figures}

Figure F-1. Infrared Absorption Spectrum of $o$-Chloropyridine F-8

Figure F-2. Proton Nuclear Magnetic Resonance Spectrum of $o$-Chloropyridine F-9

Figure F-3 Carbon-13 Nuclear Magnetic Resonance Spectrum of $o$-Chloropyridine F-10 
$o$-Chloropyridine, NTP TOX 83

\section{F.1. Procurement and Characterization}

\section{F.1.1. 0 -Chloropyridine}

$o$-Chloropyridine was obtained from Aldrich Chemical Company (Milwaukee, WI) in one lot (15306CN) that was used in the 2-week dermal and 3-month drinking water studies. Identity and purity analyses were conducted by the analytical chemistry laboratory at Battelle (Columbus, $\mathrm{OH}$, and Richland, WA), Chemir/Polytech Laboratories, Inc. (Maryland Heights, MO), Galbraith Laboratories, Inc. (Knoxville, TN), and the study laboratory, BioReliance Corporation (Rockville, MD). Reports on analyses performed in support of the $o$-chloropyridine studies are on file at the National Institute of Environmental Health Sciences.

The chemical, a clear colorless liquid, was identified as $o$-chloropyridine by the analytical chemistry laboratory, Chemir/Polytech Laboratories, Inc., and the study laboratory using infrared (IR) spectroscopy and by the analytical chemistry laboratory and Chemir/Polytech Laboratories, Inc., using proton and carbon-13 nuclear magnetic resonance (NMR) spectroscopy. All spectra were consistent with the literature spectra ${ }^{55 ; 56}$ of $o$-chloropyridine. The IR and NMR spectra are presented in Figure F-1, Figure F-2, and Figure F-3.

The purity of lot $15306 \mathrm{CN}$ was determined by Galbraith Laboratories, Inc., using elemental analyses and by the analytical chemistry laboratory using gas chromatography (GC). Elemental analyses for carbon, hydrogen, nitrogen, and chlorine were in agreement with the theoretical values for $o$-chloropyridine. GC using system A (Table F-1) indicated one major peak and two impurities with areas at least $0.1 \%$ relative to the major peak area; the purity of the bulk chemical was determined to be greater than $99 \%$. GC using system B indicated one major peak and two impurity peaks with areas at least $0.1 \%$ of the major peak area; the purity of the test chemical was determined to be greater than $99.5 \%$. The overall purity of lot $15306 \mathrm{CN}$ was determined to be $99 \%$ or greater.

To ensure stability, the bulk chemical was stored at room temperature in sealed amber glass bottles under a headspace of inert gas, protected from light and moisture. Periodic reanalyses of the bulk chemical were performed during the 2-week and 3-month studies by the study laboratory using GC by system $\mathrm{C}$, and no degradation of the bulk chemical was detected.

\section{F.1.2. Ethanol}

Ninety-five percent ethanol was obtained from Clear Spring Distilling Company (Clermont, KY) in one lot (21049312) that was used as the dosing vehicle in the 2-week dermal studies. Lot 21049312, a clear liquid, was identified as ethanol by the study laboratory using IR spectroscopy; the IR spectrum was consistent with a literature spectrum ${ }^{57}$ of ethanol.

The purity of lot 21049312 was determined by the study laboratory using GC by system D (Table F-1). Analysis indicated one major peak and three impurities each with a relative concentration of less than or equal to $0.0001 \%$. 
$o$-Chloropyridine, NTP TOX 83

\section{F.2. Preparation and Analysis of Dose Formulations}

\section{F.2.1. Dermal Studies}

The dose formulations were prepared once during the 2 -week studies by mixing $o$-chloropyridine with 95\% ethanol to give the required concentrations (Table F-2). The dose formulations were stored under a headspace of inert gas in refrigerated amber glass vials with Teflon ${ }^{\circledR}$-lined lids for up to 23 days.

Stability studies of a $3.125 \mathrm{mg} / \mathrm{mL}$ dose formulation were performed by the analytical chemistry laboratory with GC by system E (Table F-1). Stability was confirmed for at least 43 days for dose formulations stored in sealed amber glass vials at room temperature, $5^{\circ} \mathrm{C}$, or $-20^{\circ} \mathrm{C}$. Data from a simulated animal room stability study indicated that $o$-chloropyridine was stable dissolved in $95 \%$ ethanol when exposed to light for up to 3 hours at room temperature.

The dose formulations were analyzed on the day they were prepared by the study laboratory using GC by system C. All seven dose formulations analyzed for rats and mice were within 10\% of the target concentrations (Table F-3). Animal room samples of these dose formulations were also analyzed; one of five formulations for rats and all five formulations for mice were within $10 \%$ of the target concentrations. High concentrations measured for some of the rat animal room samples were attributed to improper sealing of the vials after dosing and possible solvent evaporation.

\section{F.2.2. Drinking Water Studies}

The dose formulations were prepared nine times during the 3-month studies by mixing $o$ chloropyridine with tap water to give the required concentrations (Table F-2). The dose formulations were stored refrigerated in Cubitainers ${ }^{\circledR}$ with taps, protected from light, for up to 21 days.

Because all dose formulations in these studies were determined to be solutions, no homogeneity studies were required. Stability studies of the $10 \mathrm{ppm}$ dose formulation were performed by the analytical chemistry laboratory with high-performance liquid chromatography (HPLC). The analytical system consisted of a Waters (Waters Corporation, Milford, MA) or Spectra-Physics (Spectra-Physics, Inc., Mountain View, CA) liquid chromatograph, a Luna ${ }^{\circledR}$ (5 $\mu \mathrm{m}$ particle size, C18 $150 \mathrm{~mm} \times 4.6 \mathrm{~mm}$ ) column (Phenomenex, Inc., Torrance, CA), an isocratic mobile phase of acetonitrile:Milli- $\mathrm{Q}^{\circledR}$ water:glacial acetic acid $(33: 66: 1)$ at a flow rate of $1.0 \mathrm{~mL} / \mathrm{minute}$, and ultraviolet detection at $254 \mathrm{~nm}$. Stability was confirmed for at least 43 days for dose formulations stored in sealed polyethylene bottles protected from light at $5^{\circ} \mathrm{C}$ and for at least 8 days under simulated animal room conditions.

The dose formulations were analyzed at the beginning, midpoint, and end of the studies by the study laboratory using the HPLC system described above for the dose formulation stability studies; animal room samples of these dose formulations were also analyzed. All 15 dose formulations analyzed and used for rats and mice were within $10 \%$ of the target concentrations (Table F-4). Of the animal room samples analyzed, all 15 for rats and 12 of 15 for mice were within $10 \%$ of the target concentrations, and the remaining three were less than $12 \%$ of the target concentrations. 
Table F-1. Gas Chromatography Systems Used in the Studies of $o$-Chloropyridine ${ }^{\text {a }}$

\begin{tabular}{|c|c|c|}
\hline Detection System & Column & Oven Temperature Program \\
\hline \multicolumn{3}{|l|}{ System A } \\
\hline Flame ionization & $\begin{array}{l}\text { Stabilwax }{ }^{\circledR}, 30 \mathrm{~m} \times 0.53 \mathrm{~mm}, \\
1.0 \mu \mathrm{m} \text { film (Restek, Bellefonte, PA) }\end{array}$ & $\begin{array}{l}65^{\circ} \mathrm{C} \text { for } 1 \text { minute, then } 5^{\circ} \mathrm{C} / \text { minute to } \\
230^{\circ} \mathrm{C} \text {, held for } 1 \text { minute }\end{array}$ \\
\hline \multicolumn{3}{|l|}{ System B } \\
\hline Flame ionization & $\begin{array}{l}\text { Stabilwax }{ }^{\circledR}-\mathrm{DB}, 30 \mathrm{~m} \times 0.25 \mathrm{~mm}, \\
0.5 \mu \mathrm{m} \text { film }(\text { Restek })\end{array}$ & $\begin{array}{l}50^{\circ} \mathrm{C} \text { for } 1 \text { minute, then } 10^{\circ} \mathrm{C} / \text { minute to } \\
200^{\circ} \mathrm{C} \text {, held for } 13 \text { minutes }\end{array}$ \\
\hline \multicolumn{3}{|l|}{ System C } \\
\hline Flame ionization & $\begin{array}{l}\mathrm{DB}^{\mathrm{TM}}-\mathrm{WAX}, 30 \mathrm{~m} \times 0.53 \mathrm{~mm}, \\
1.0 \mu \mathrm{m} \text { film }(\mathrm{J} \& \mathrm{~W} \text { Scientific, } \\
\text { Folsom, CA })\end{array}$ & $\begin{array}{l}75^{\circ} \mathrm{C} \text { for } 1 \text { minute, then } 7^{\circ} \mathrm{C} / \text { minute to } \\
180^{\circ} \mathrm{C} \text {, then } 15^{\circ} \mathrm{C} / \text { minute to } 230^{\circ} \mathrm{C} \text {, held } \\
\text { for } 1 \text { minute }\end{array}$ \\
\hline \multicolumn{3}{|l|}{ System D } \\
\hline Flame ionization & $\begin{array}{l}\mathrm{DB}^{\mathrm{TM}}-\mathrm{WAX}, 30 \mathrm{~m} \times 0.53 \mathrm{~mm} \\
1.0 \mu \mathrm{m} \text { film, J\&W Scientific }\end{array}$ & $\begin{array}{l}40^{\circ} \mathrm{C} \text { for } 5 \text { minutes, then } 10^{\circ} \mathrm{C} / \text { minute to } \\
220^{\circ} \mathrm{C} \text {, held for } 5 \text { minutes }\end{array}$ \\
\hline \multicolumn{3}{|l|}{ System E } \\
\hline Flame ionization & $\begin{array}{l}\text { Stabilwax }{ }^{\circledR}-\mathrm{DB}, 30 \mathrm{~m} \times 0.25 \mathrm{~mm}, \\
0.5 \mu \mathrm{m} \text { film }(\text { Restek })\end{array}$ & $\begin{array}{l}75^{\circ} \mathrm{C} \text { for } 1 \text { minute, then } 7^{\circ} \mathrm{C} / \text { minute to } \\
180^{\circ} \mathrm{C} \text {, then } 15^{\circ} \mathrm{C} / \text { minute to } 230^{\circ} \mathrm{C} \text {, held } \\
\text { for } 1 \text { minute }\end{array}$ \\
\hline
\end{tabular}

${ }^{\mathrm{a} A l l}$ gas chromatographs were manufactured by Hewlett-Packard, (Palo Alto, CA).

Table F-2. Preparation and Storage of Dose Formulations in the Studies of $o$-Chloropyridine

Two-week Dermal Studies

\section{Preparation}

Each dose formulation was prepared by diluting a weighed amount of $o$-chloropyridine with a specified volume of $95 \%$ ethanol in a graduated cylinder. The dose formulations were stirred on a magnetic stirrer until the mixtures were homogenized. Dose formulations were prepared once during the studies.

\section{Chemical Lot Number}

$15306 \mathrm{CN}$

\section{Maximum Storage Time}

23 days

\section{Storage Conditions}

The dose formulations were stored in amber glass vials under a headspace of inert gas; the vials were sealed with Teflon ${ }^{\circledR}$-lined septa and crimped aluminum caps and were stored refrigerated.

\section{Study Laboratory}

BioReliance Corporation (Rockville, MD)

\section{Three-month Drinking Water Studies}

Tap water was weighed to a volume of $1 \mathrm{~L}$ less than the final volume of the formulation into a Cubitainer ${ }^{\circledR}$. Water was removed from the Cubitainer ${ }^{\circledR}$ to account for the volume of $o$-chloropyridine to be added.

$o$-Chloropyridine was weighed into a tared glass beaker and the contents were quantitatively transferred to the Cubitainer ${ }^{\circledR}$ using $1 \mathrm{~L}$ of tap water measured in a beaker. The final mixture was agitated by vigorous shaking. Dose formulations were prepared nine times.

\section{$15306 \mathrm{CN}$}

21 days

Dose formulations were stored in 20 L Cubitainers ${ }^{\circledR}$ with taps, refrigerated and protected from light. 
Table F-3. Results of Analyses of Dose Formulations Administered to Rats and Mice in the Two-week Dermal Studies of $o$-Chloropyridine

\begin{tabular}{|c|c|c|c|c|}
\hline Date Prepared & Date Analyzed & $\begin{array}{c}\text { Target } \\
\text { Concentration } \\
(\mathrm{mg} / \mathrm{mL})\end{array}$ & $\begin{array}{c}\text { Determined } \\
\text { Concentration } \\
(\mathbf{m g} / \mathrm{mL})\end{array}$ & $\begin{array}{c}\text { Difference from } \\
\text { Target }(\%)\end{array}$ \\
\hline \multirow[t]{17}{*}{ September 12, 2000} & September 12, 2000 & 3.13 & 3.20 & +7 \\
\hline & & 6.25 & 6.31 & +1 \\
\hline & & 12.5 & 12.4 & -1 \\
\hline & & 25 & 25.1 & 0 \\
\hline & & 50 & 49.0 & -2 \\
\hline & & 100 & 98.0 & -2 \\
\hline & & 200 & 194 & -3 \\
\hline & October $11,2000^{c}$ & 12.5 & 13.5 & +8 \\
\hline & & 25 & 29.2 & +17 \\
\hline & & 50 & 55.3 & +11 \\
\hline & & 100 & 111 & +11 \\
\hline & & 200 & 236 & +18 \\
\hline & October $11,2000^{\mathrm{d}}$ & 3.13 & 3.22 & +3 \\
\hline & & 6.25 & 6.38 & +2 \\
\hline & & 12.5 & 12.9 & +3 \\
\hline & & 25 & 26.5 & +6 \\
\hline & & 50 & 53.1 & +6 \\
\hline
\end{tabular}

${ }^{\text {a The }} 3.13$ and $6.25 \mathrm{mg} / \mathrm{mL}$ dose formulations were used only for mice, and the 100 and $200 \mathrm{mg} / \mathrm{mL}$ dose formulations were used only for rats.

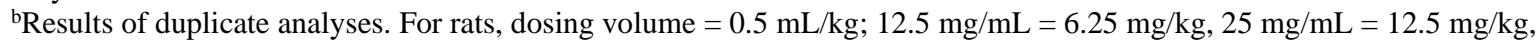

$50 \mathrm{mg} / \mathrm{mL}=25 \mathrm{mg} / \mathrm{kg} ; 100 \mathrm{mg} / \mathrm{mL}=50 \mathrm{mg} / \mathrm{kg} ; 200 \mathrm{mg} / \mathrm{mL}=100 \mathrm{mg} / \mathrm{kg}$. For mice, dosing volume $=2 \mathrm{~mL} / \mathrm{kg}$;

$3.13 \mathrm{mg} / \mathrm{mL}=6.25 \mathrm{mg} / \mathrm{kg}, 6.25 \mathrm{mg} / \mathrm{mL}=12.5 \mathrm{mg} / \mathrm{kg}, 12.5 \mathrm{mg} / \mathrm{mL}=25 \mathrm{mg} / \mathrm{kg} ; 25 \mathrm{mg} / \mathrm{mL}=50 \mathrm{mg} / \mathrm{kg}, 50 \mathrm{mg} / \mathrm{mL}=100 \mathrm{mg} / \mathrm{kg}$.

${ }^{\mathrm{c}}$ Animal room samples for rats.

${ }^{\mathrm{d}}$ Animal room samples for mice. 
Table F-4. Results of Analyses of Dose Formulations Administered to Rats and Mice in the Three-month Drinking Water Studies of $o$-Chloropyridine

\begin{tabular}{|c|c|c|c|c|}
\hline Date Prepared & Date Analyzed & $\begin{array}{c}\text { Target } \\
\text { Concentration } \\
(\text { ppm })\end{array}$ & $\begin{array}{c}\text { Determined } \\
\text { Concentration } \\
(\mathbf{p p m})\end{array}$ & $\begin{array}{c}\text { Difference from } \\
\text { Target }(\%)\end{array}$ \\
\hline \multirow[t]{13}{*}{ July 22, 2003} & July 22, 2003 & 10 & 9.76 & -2 \\
\hline & & 30 & 29.7 & -1 \\
\hline & & 100 & 97.9 & -2 \\
\hline & & 300 & 300 & 0 \\
\hline & & 1,000 & $897^{\mathrm{b}}$ & -10 \\
\hline & August $7,2003^{c}$ & 10 & 9.23 & -8 \\
\hline & & 30 & 27.9 & -7 \\
\hline & & 100 & 94.2 & -6 \\
\hline & & 300 & 278 & -7 \\
\hline & August $7,2003^{\mathrm{d}}$ & 10 & 8.79 & -12 \\
\hline & & 30 & 26.7 & -11 \\
\hline & & 100 & 93.0 & -7 \\
\hline & & 300 & 270 & -10 \\
\hline \multirow[t]{3}{*}{ July 25, 2003} & July 25, 2003 & 1,000 & $982^{\mathrm{e}}$ & -2 \\
\hline & August $7,2003^{c}$ & 1,000 & 936 & -6 \\
\hline & August $7,2003^{\mathrm{d}}$ & 1,000 & 905 & -10 \\
\hline \multirow[t]{15}{*}{ August 25, 2003} & August 26, 2003 & 10 & 9.92 & -1 \\
\hline & & 30 & 28.8 & -4 \\
\hline & & 100 & 98.1 & -2 \\
\hline & & 300 & 296 & -1 \\
\hline & & 1,000 & 992 & -1 \\
\hline & September $17,2003^{c}$ & 10 & 9.33 & -7 \\
\hline & & 30 & 28.0 & -7 \\
\hline & & 100 & 92.0 & -8 \\
\hline & & 300 & 282 & -6 \\
\hline & & 1,000 & 944 & -6 \\
\hline & September $17,2003^{\mathrm{d}}$ & 10 & 9.16 & -8 \\
\hline & & 30 & 27.1 & -10 \\
\hline & & 100 & 92.1 & -8 \\
\hline & & 300 & 279 & -7 \\
\hline & & 1,000 & 889 & -11 \\
\hline \multirow[t]{2}{*}{ October 14, 2003} & October 16, 2003 & 10 & 9.54 & -5 \\
\hline & & 30 & 28.8 & -4 \\
\hline
\end{tabular}


$o$-Chloropyridine, NTP TOX 83

\begin{tabular}{|c|c|c|c|c|}
\hline Date Prepared & Date Analyzed & $\begin{array}{c}\text { Target } \\
\text { Concentration } \\
(\mathbf{p p m})\end{array}$ & $\begin{array}{c}\text { Determined } \\
\text { Concentration } \\
(\mathbf{p p m})\end{array}$ & $\begin{array}{c}\text { Difference from } \\
\text { Target }(\%)\end{array}$ \\
\hline & \multirow{7}{*}{ November $5,2003^{c}$} & 100 & 98.0 & -2 \\
\hline & & 300 & 290 & -3 \\
\hline & & 1,000 & 974 & -3 \\
\hline & & 10 & 9.59 & -4 \\
\hline & & 30 & 28.3 & -6 \\
\hline & & 100 & 94.6 & -5 \\
\hline & & 300 & 278 & -7 \\
\hline & \multirow{6}{*}{ November $5,2003^{\mathrm{d}}$} & 1,000 & 930 & -7 \\
\hline & & 10 & 9.31 & -7 \\
\hline & & 30 & 28.0 & -7 \\
\hline & & 100 & 95.1 & -5 \\
\hline & & 300 & 269 & -10 \\
\hline & & 1,000 & 914 & -9 \\
\hline
\end{tabular}

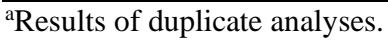

${ }^{b}$ Remixed; not used in studies.

${ }^{\mathrm{c}}$ Animal room samples for rats.

${ }^{\mathrm{d}}$ Animal room samples for mice.

${ }^{\mathrm{e}}$ Results of remix. 


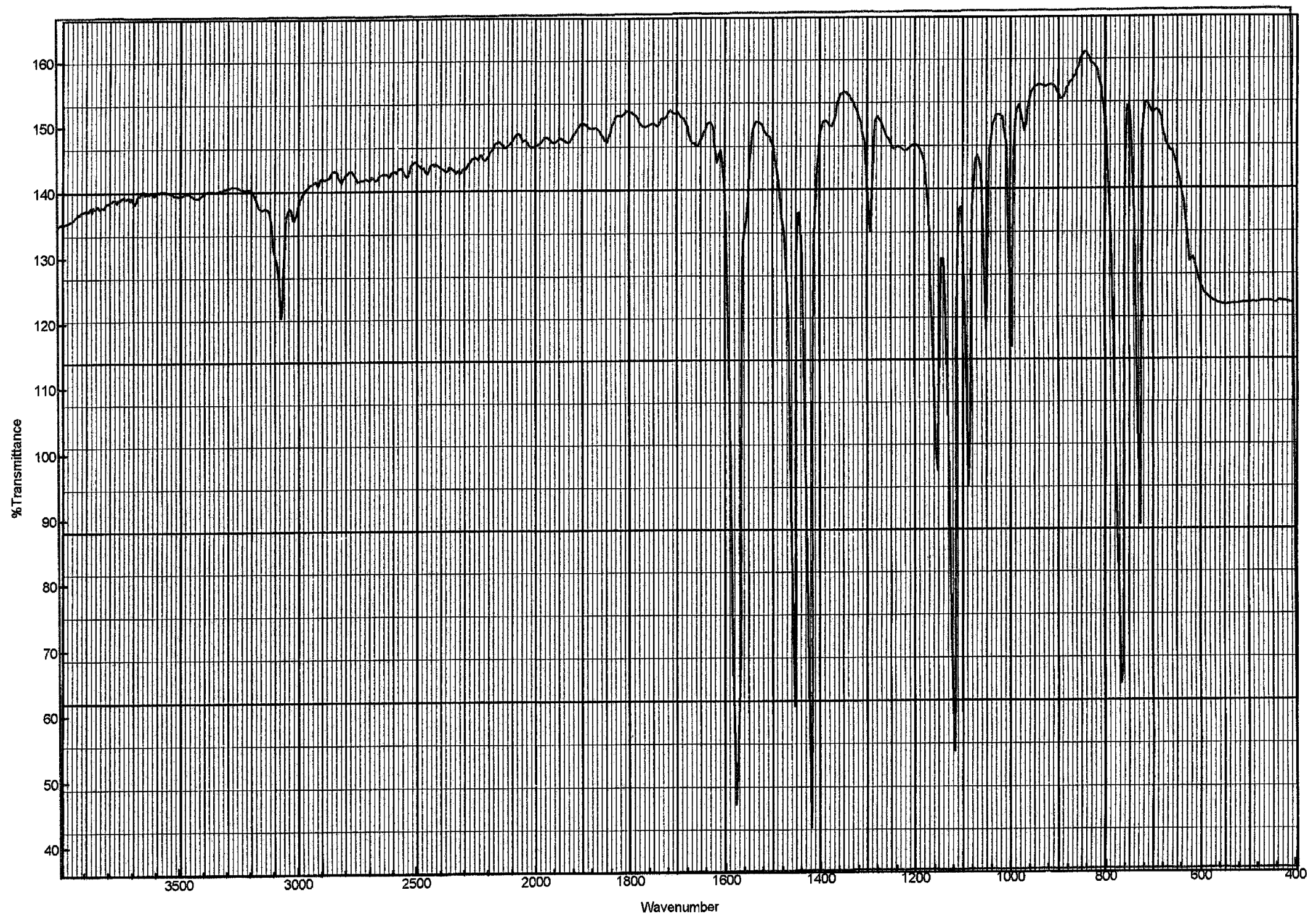

Figure F-1. Infrared Absorption Spectrum of o-Chloropyridine 


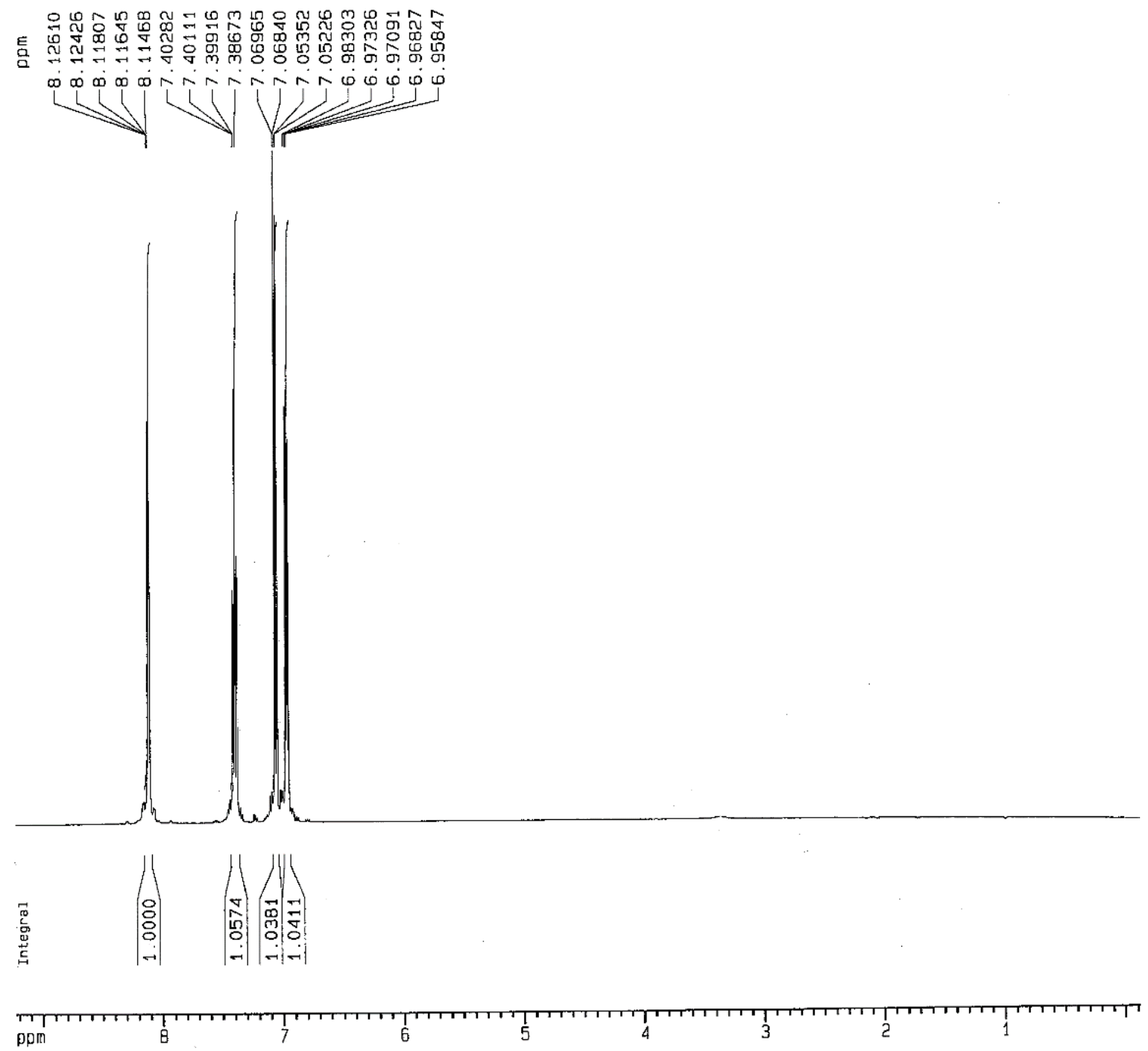

Figure F-2. Proton Nuclear Magnetic Resonance Spectrum of $o$-Chloropyridine 
$o$-Chloropyridine, NTP TOX 83

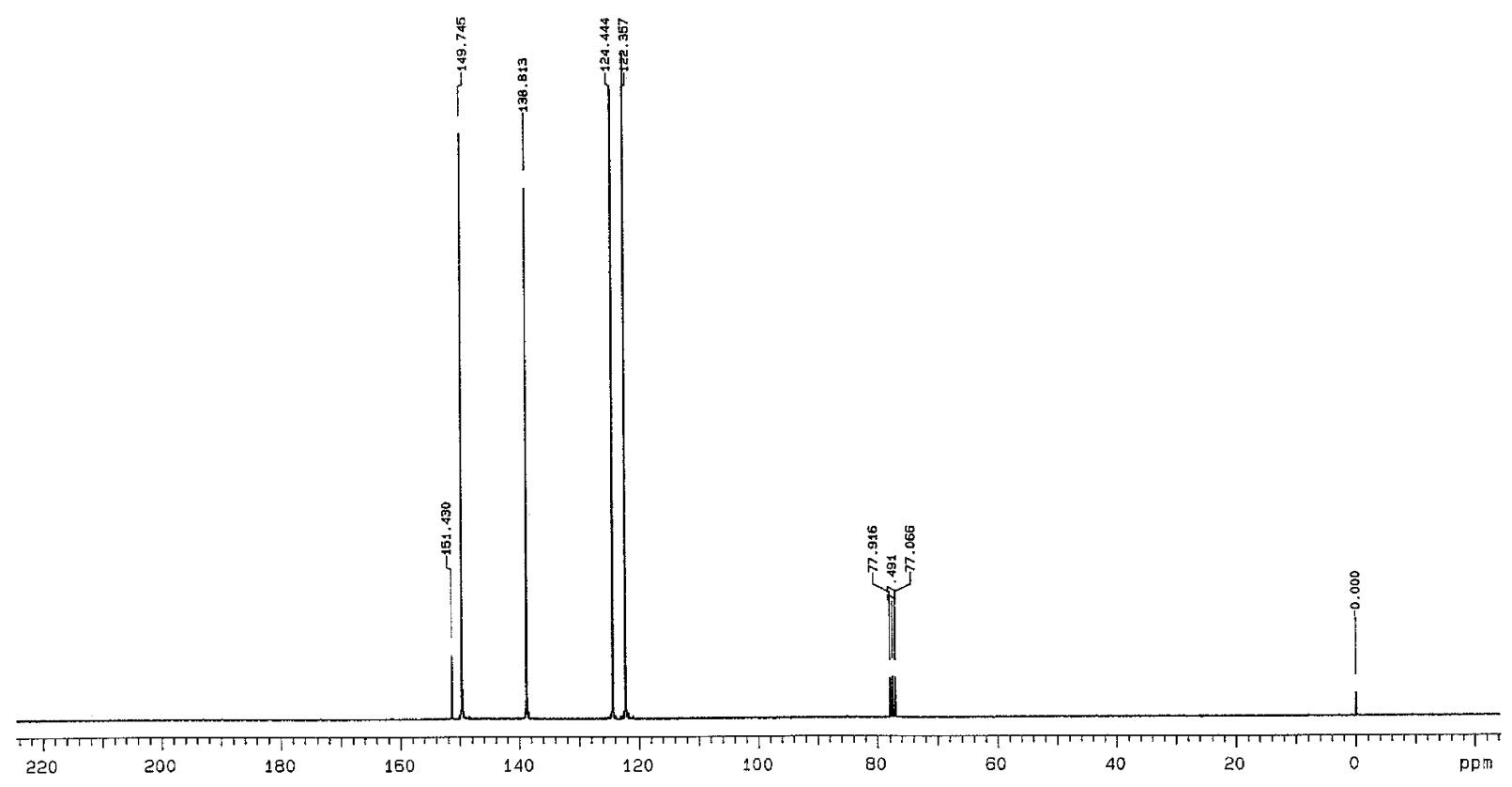

Figure F-3 Carbon-13 Nuclear Magnetic Resonance Spectrum of o-Chloropyridine 


\section{Appendix G. Water and Compound Consumption in the Three-month Drinking Water Studies of o-Chloropyridine}

\section{Tables}

Table G-1. Water and Compound Consumption by Male Rats in the Three-month

Drinking Water Study of $o$-Chloropyridine . G-2

Table G-2. Water and Compound Consumption by Female Rats in the Three-month

Drinking Water Study of $o$-Chloropyridine G-3

Table G-3. Water and Compound Consumption by Male Mice in the Three-month Drinking Water Study of $o$-Chloropyridine G-4

Table G-4. Water and Compound Consumption by Female Mice in the Three-month Drinking Water Study of $o$-Chloropyridine G-5 
$o$-Chloropyridine, NTP TOX 83

Table G-1. Water and Compound Consumption by Male Rats in the Three-month Drinking Water Study of $o$-Chloropyridine

\begin{tabular}{|c|c|c|c|c|c|c|c|c|c|c|c|}
\hline \multirow[b]{2}{*}{ Week } & \multicolumn{2}{|c|}{0 ppm } & \multicolumn{3}{|c|}{10 ppm } & \multicolumn{3}{|c|}{30 ppm } & \multicolumn{3}{|c|}{100 ppm } \\
\hline & $\begin{array}{l}\text { Water } \\
\text { (g/day) }\end{array}$ & $\begin{array}{c}\text { Body } \\
\text { Weight (g) }\end{array}$ & $\begin{array}{c}\text { Water } \\
\text { (g/day) }\end{array}$ & $\begin{array}{c}\text { Body } \\
\text { Weight (g) }\end{array}$ & $\begin{array}{c}\text { Dose }^{\mathrm{b}} \\
(\mathrm{mg} / \mathrm{kg})\end{array}$ & $\begin{array}{l}\text { Water } \\
\text { (g/day) }\end{array}$ & $\begin{array}{c}\text { Body } \\
\text { Weight (g) }\end{array}$ & $\begin{array}{c}\text { Dose } \\
(\mathrm{mg} / \mathrm{kg})\end{array}$ & $\begin{array}{r}\text { Water } \\
\text { (g/day) }\end{array}$ & $\begin{array}{c}\text { Body } \\
\text { Weight (g) }\end{array}$ & $\begin{array}{c}\text { Dose } \\
(\mathrm{mg} / \mathrm{kg})\end{array}$ \\
\hline 1 & 14.7 & 90 & 15.5 & 93 & 1.7 & 15.1 & 92 & 4.9 & 15.4 & 91 & 16.9 \\
\hline 2 & 14.9 & 119 & 16.6 & 123 & 1.3 & 15.8 & 123 & 3.8 & 15.4 & 120 & 12.9 \\
\hline 3 & 16.6 & 146 & 17.7 & 152 & 1.2 & 18.3 & 152 & 3.6 & 15.7 & 148 & 10.6 \\
\hline 4 & 17.2 & 175 & 18.3 & 182 & 1.0 & 18.8 & 184 & 3.1 & 16.6 & 174 & 9.6 \\
\hline 5 & 17.3 & 199 & 17.6 & 205 & 0.9 & 18.4 & 209 & 2.6 & 18.8 & 195 & 9.6 \\
\hline 6 & 15.7 & 217 & 16.6 & 225 & 0.7 & 17.2 & 230 & 2.2 & 20.2 & 213 & 9.5 \\
\hline 7 & 16.5 & 234 & 19.3 & 239 & 0.8 & 18.9 & 247 & 2.3 & 16.8 & 233 & 7.2 \\
\hline 8 & 16.2 & 247 & 17.1 & 254 & 0.7 & 21.7 & 263 & 2.5 & 17.1 & 247 & 6.9 \\
\hline 9 & 16.4 & 262 & 16.5 & 268 & 0.6 & 18.0 & 278 & 1.9 & 18.0 & 260 & 6.9 \\
\hline 10 & 16.0 & 275 & 15.9 & 281 & 0.6 & 19.0 & 295 & 1.9 & 19.5 & 277 & 7.0 \\
\hline 11 & 16.3 & 285 & 16.0 & 291 & 0.6 & 17.3 & 305 & 1.7 & 19.7 & 289 & 6.8 \\
\hline 12 & 16.9 & 297 & 17.0 & 302 & 0.6 & 17.3 & 316 & 1.6 & 19.6 & 305 & 6.4 \\
\hline 13 & 16.8 & 306 & 16.2 & 311 & 0.5 & 18.8 & 324 & 1.7 & 18.1 & 312 & 5.8 \\
\hline \multirow[t]{3}{*}{14} & 17.3 & 320 & 17.7 & 326 & 0.5 & 13.6 & 326 & 1.3 & 18.1 & 326 & 5.6 \\
\hline & & & & & & \multicolumn{3}{|c|}{300 ppm } & \multicolumn{3}{|c|}{1,000 ppm } \\
\hline & & & & & & $\begin{array}{c}\text { Water } \\
\text { (g/day) }\end{array}$ & $\begin{array}{c}\text { Body } \\
\text { Weight (g) }\end{array}$ & $\begin{array}{c}\text { Dose } \\
\text { (mg/kg) }\end{array}$ & $\begin{array}{r}\text { Water } \\
\text { (g/day) }\end{array}$ & $\begin{array}{c}\text { Body } \\
\text { Weight (g) }\end{array}$ & $\begin{array}{c}\text { Dose } \\
(\mathrm{mg} / \mathrm{kg})\end{array}$ \\
\hline 1 & & & & & & 13.6 & 91 & 44.9 & 6.5 & 94 & 68.9 \\
\hline 2 & & & & & & 15.2 & 118 & 38.6 & 8.8 & 98 & 89.6 \\
\hline 3 & & & & & & 16.6 & 145 & 34.3 & 10.2 & 121 & 84.5 \\
\hline 4 & & & & & & 16.9 & 172 & 29.5 & 11.3 & 145 & 77.9 \\
\hline 5 & & & & & & 16.5 & 192 & 25.8 & 12.0 & 164 & 73.0 \\
\hline 6 & & & & & & 15.2 & 210 & 21.7 & 11.3 & 182 & 62.1 \\
\hline 7 & & & & & & 17.7 & 222 & 24.0 & 11.7 & 195 & 60.0 \\
\hline 8 & & & & & & 16.9 & 239 & 21.2 & 11.6 & 209 & 55.4 \\
\hline 9 & & & & & & 16.0 & 255 & 18.9 & 12.8 & 222 & 57.7 \\
\hline 10 & & & & & & 15.8 & 267 & 17.8 & 13.0 & 219 & 59.4 \\
\hline 11 & & & & & & 16.1 & 275 & 17.6 & 12.7 & 235 & 54.0 \\
\hline 12 & & & & & & 16.4 & 286 & 17.2 & 12.8 & 249 & 51.4 \\
\hline 13 & & & & & & 16.1 & 293 & 16.5 & 13.0 & 258 & 50.4 \\
\hline 14 & & & & & & 17.5 & 309 & 17.0 & 15.1 & 268 & 56.4 \\
\hline
\end{tabular}

${ }^{\mathrm{a}}$ Grams of water consumed per animal per day.

${ }^{b}$ Milligrams of $o$-chloropyridine consumed per kilogram body weight per day. 
$o$-Chloropyridine, NTP TOX 83

Table G-2. Water and Compound Consumption by Female Rats in the Three-month Drinking Water Study of $o$-Chloropyridine

\begin{tabular}{|c|c|c|c|c|c|c|c|c|c|c|c|}
\hline \multirow[b]{2}{*}{ Week } & \multicolumn{2}{|c|}{ 0 ppm } & \multicolumn{3}{|c|}{$10 \mathrm{ppm}$} & \multicolumn{3}{|c|}{30 ppm } & \multicolumn{3}{|c|}{100 ppm } \\
\hline & $\begin{array}{l}\text { Water } \\
\text { (g/day) }\end{array}$ & $\begin{array}{c}\text { Body } \\
\text { Weight } \\
\text { (g) }\end{array}$ & $\begin{array}{l}\text { Water } \\
\text { (g/day) }\end{array}$ & $\begin{array}{l}\text { Body } \\
\text { Weight } \\
\text { (g) }\end{array}$ & $\begin{array}{c}\text { Dose }^{\mathrm{b}} \\
(\mathrm{mg} / \mathrm{kg})\end{array}$ & $\begin{array}{l}\text { Water } \\
\text { (g/day) }\end{array}$ & $\begin{array}{c}\text { Body } \\
\text { Weight } \\
\text { (g) }\end{array}$ & $\begin{array}{c}\text { Dose } \\
(\mathrm{mg} / \mathrm{kg})\end{array}$ & $\begin{array}{l}\text { Water } \\
\text { (g/day) }\end{array}$ & $\begin{array}{c}\text { Body } \\
\text { Weight } \\
\text { (g) }\end{array}$ & $\begin{array}{c}\text { Dose } \\
(\mathrm{mg} / \mathrm{kg})\end{array}$ \\
\hline 1 & 12.5 & 86 & 13.8 & 88 & 1.6 & 13.0 & 87 & 4.5 & 12.6 & 88 & 14.3 \\
\hline 2 & 13.4 & 106 & 13.8 & 108 & 1.3 & 13.6 & 108 & 3.8 & 13.4 & 108 & 12.5 \\
\hline 3 & 13.9 & 120 & 14.1 & 122 & 1.2 & 15.8 & 123 & 3.8 & 13.5 & 122 & 11.1 \\
\hline 4 & 14.4 & 135 & 14.8 & 137 & 1.1 & 15.5 & 139 & 3.3 & 13.8 & 137 & 10.1 \\
\hline 5 & 15.5 & 146 & 15.7 & 149 & 1.1 & 15.7 & 150 & 3.1 & 12.4 & 149 & 8.3 \\
\hline 6 & 14.0 & 158 & 15.7 & 161 & 1.0 & 14.9 & 160 & 2.8 & 15.2 & 161 & 9.5 \\
\hline 7 & 14.0 & 168 & 14.4 & 171 & 0.8 & 16.2 & 169 & 2.9 & 13.8 & 170 & 8.1 \\
\hline 8 & 13.5 & 175 & 14.0 & 177 & 0.8 & 16.3 & 176 & 2.8 & 13.3 & 176 & 7.6 \\
\hline 9 & 12.4 & 179 & 13.4 & 181 & 0.7 & 13.8 & 180 & 2.3 & 12.9 & 179 & 7.2 \\
\hline 10 & 13.0 & 184 & 13.6 & 185 & 0.7 & 14.4 & 185 & 2.3 & 12.7 & 183 & 6.9 \\
\hline 11 & 14.3 & 188 & 13.5 & 190 & 0.7 & 14.6 & 190 & 2.3 & 14.5 & 189 & 7.7 \\
\hline 12 & 14.2 & 191 & 13.9 & 194 & 0.7 & 14.1 & 195 & 2.2 & 14.5 & 192 & 7.6 \\
\hline 13 & 14.1 & 192 & 13.8 & 196 & 0.7 & 15.2 & 195 & 2.3 & 12.8 & 194 & 6.6 \\
\hline \multirow[t]{3}{*}{14} & 13.4 & 195 & 17.7 & 200 & 0.9 & 14.8 & 200 & 2.2 & 17.4 & 196 & 8.9 \\
\hline & & & & & & \multicolumn{3}{|c|}{$300 \mathrm{ppm}$} & \multicolumn{3}{|c|}{1,000 ppm } \\
\hline & & & & & & $\begin{array}{l}\text { Water } \\
\text { (g/day) }\end{array}$ & $\begin{array}{l}\text { Body } \\
\text { Weight } \\
\text { (g) }\end{array}$ & $\begin{array}{c}\text { Dose } \\
(\mathrm{mg} / \mathrm{kg})\end{array}$ & $\begin{array}{l}\text { Water } \\
\text { (g/day) }\end{array}$ & $\begin{array}{l}\text { Body } \\
\text { Weight } \\
\text { (g) }\end{array}$ & $\begin{array}{c}\text { Dose } \\
(\mathrm{mg} / \mathrm{kg})\end{array}$ \\
\hline 1 & & & & & & 12.2 & 86 & 42.6 & 6.1 & 87 & 70.1 \\
\hline 2 & & & & & & 13.1 & 105 & 37.6 & 7.7 & 89 & 86.1 \\
\hline 3 & & & & & & 13.3 & 119 & 33.7 & 8.7 & 105 & 83.1 \\
\hline 4 & & & & & & 13.6 & 132 & 30.9 & 9.4 & 119 & 78.9 \\
\hline 5 & & & & & & 13.7 & 145 & 28.4 & 10.0 & 130 & 77.0 \\
\hline 6 & & & & & & 13.1 & 155 & 25.4 & 10.0 & 139 & 71.9 \\
\hline 7 & & & & & & 12.7 & 163 & 23.4 & 9.8 & 148 & 66.4 \\
\hline 8 & & & & & & 12.8 & 168 & 22.8 & 9.8 & 154 & 63.7 \\
\hline 9 & & & & & & 12.2 & 173 & 21.2 & 9.8 & 158 & 62.0 \\
\hline 10 & & & & & & 12.6 & 176 & 21.5 & 10.1 & 163 & 61.9 \\
\hline 11 & & & & & & 12.2 & 182 & 20.1 & 10.3 & 167 & 61.6 \\
\hline 12 & & & & & & 12.3 & 185 & 20.0 & 10.5 & 170 & 61.6 \\
\hline 13 & & & & & & 13.0 & 187 & 20.8 & 10.4 & 173 & 60.2 \\
\hline 14 & & & & & & 12.7 & 190 & 20.0 & 15.8 & 174 & 90.9 \\
\hline
\end{tabular}

${ }^{\mathrm{a}}$ Grams of water consumed per animal per day.

${ }^{b}$ Milligrams of $o$-chloropyridine consumed per kilogram body weight per day. 
$o$-Chloropyridine, NTP TOX 83

Table G-3. Water and Compound Consumption by Male Mice in the Three-month Drinking Water Study of $o$-Chloropyridine

\begin{tabular}{|c|c|c|c|c|c|c|c|c|c|c|c|}
\hline \multirow[b]{2}{*}{ Week } & \multicolumn{2}{|c|}{ O ppm } & \multicolumn{3}{|c|}{10 ppm } & \multicolumn{3}{|c|}{30 ppm } & \multicolumn{3}{|c|}{100 ppm } \\
\hline & $\begin{array}{l}\text { Water } \\
\text { (g/day) }\end{array}$ & $\begin{array}{c}\text { Body } \\
\text { Weight (g) }\end{array}$ & $\begin{array}{c}\text { Water } \\
\text { (g/day) }\end{array}$ & $\begin{array}{c}\text { Body } \\
\text { Weight (g) }\end{array}$ & $\begin{array}{c}\text { Dose }^{\mathrm{b}} \\
(\mathrm{mg} / \mathrm{kg})\end{array}$ & $\begin{array}{c}\text { Water } \\
\text { (g/day) }\end{array}$ & $\begin{array}{c}\text { Body } \\
\text { Weight (g) }\end{array}$ & $\begin{array}{c}\text { Dose } \\
(\mathrm{mg} / \mathrm{kg})\end{array}$ & $\begin{array}{l}\text { Water } \\
\text { (g/day) }\end{array}$ & $\begin{array}{c}\text { Body } \\
\text { Weight (g) }\end{array}$ & $\begin{array}{c}\text { Dose } \\
(\mathrm{mg} / \mathrm{kg})\end{array}$ \\
\hline 1 & 4.8 & 23.2 & 5.1 & 23.2 & 2.2 & 5.4 & 23.3 & 7.0 & 5.3 & 23.3 & 22.7 \\
\hline 2 & 4.9 & 25.9 & 5.2 & 26.2 & 2.0 & 5.4 & 26.2 & 6.2 & 5.1 & 26.0 & 19.6 \\
\hline 3 & 4.6 & 27.5 & 4.8 & 27.6 & 1.7 & 5.0 & 28.1 & 5.3 & 4.5 & 27.7 & 16.2 \\
\hline 4 & 4.5 & 29.0 & 4.8 & 29.4 & 1.6 & 4.5 & 29.9 & 4.5 & 4.5 & 29.4 & 15.3 \\
\hline 5 & 4.9 & 30.5 & 5.1 & 31.1 & 1.6 & 5.0 & 31.6 & 4.7 & 4.7 & 30.9 & 15.2 \\
\hline 6 & 4.3 & 31.9 & 4.6 & 32.9 & 1.4 & 4.7 & 33.3 & 4.2 & 4.8 & 32.1 & 15.0 \\
\hline 7 & 4.8 & 33.9 & 4.8 & 34.4 & 1.4 & 4.9 & 34.8 & 4.2 & 4.5 & 33.4 & 13.5 \\
\hline 8 & 5.1 & 35.4 & 5.1 & 36.2 & 1.4 & 4.9 & 36.4 & 4.0 & 5.1 & 34.8 & 14.7 \\
\hline 9 & 4.1 & 36.5 & 4.8 & 37.4 & 1.3 & 4.8 & 37.6 & 3.8 & 4.8 & 36.1 & 13.3 \\
\hline 10 & 4.6 & 37.5 & 4.5 & 38.4 & 1.2 & 4.6 & 38.6 & 3.6 & 4.5 & 37.1 & 12.1 \\
\hline 11 & 4.7 & 37.5 & 4.8 & 38.9 & 1.2 & 4.4 & 38.7 & 3.4 & 4.5 & 37.5 & 12.0 \\
\hline 12 & 5.0 & 38.8 & 5.1 & 40.3 & 1.3 & 4.9 & 40.1 & 3.7 & 5.0 & 38.2 & 13.1 \\
\hline 13 & 5.1 & 40.1 & 5.2 & 41.7 & 1.2 & 5.2 & 41.2 & 3.8 & 4.8 & 39.3 & 12.2 \\
\hline \multirow[t]{3}{*}{14} & 4.5 & 40.8 & 4.6 & 42.4 & 1.1 & 4.9 & 42.4 & 3.5 & 5.4 & 40.2 & 13.4 \\
\hline & & & & & & \multicolumn{3}{|c|}{300 ppm } & \multicolumn{3}{|c|}{$1,000 \mathrm{ppm}$} \\
\hline & & & & & & $\begin{array}{c}\text { Water } \\
\text { (g/day) }\end{array}$ & $\begin{array}{c}\text { Body } \\
\text { Weight (g) }\end{array}$ & $\begin{array}{c}\text { Dose } \\
(\mathrm{mg} / \mathrm{kg})\end{array}$ & $\begin{array}{l}\text { Water } \\
\text { (g/day) }\end{array}$ & $\begin{array}{c}\text { Body } \\
\text { Weight (g) }\end{array}$ & $\begin{array}{c}\text { Dose } \\
(\mathrm{mg} / \mathrm{kg})\end{array}$ \\
\hline 1 & & & & & & 4.4 & 23.1 & 57.1 & 3.2 & 23.1 & 138.3 \\
\hline 2 & & & & & & 4.5 & 25.9 & 52.1 & 2.8 & 24.6 & 113.8 \\
\hline 3 & & & & & & 4.4 & 27.8 & 47.4 & 3.0 & 26.0 & 115.4 \\
\hline 4 & & & & & & 4.3 & 29.4 & 43.9 & 2.9 & 26.8 & 108.4 \\
\hline 5 & & & & & & 4.4 & 30.8 & 42.8 & 3.2 & 27.7 & 115.5 \\
\hline 6 & & & & & & 4.2 & 32.5 & 38.8 & 3.2 & 28.8 & 111.3 \\
\hline 7 & & & & & & 4.2 & 34.0 & 37.1 & 3.3 & 29.7 & 111.1 \\
\hline 8 & & & & & & 4.7 & 35.6 & 39.6 & 3.4 & 30.5 & 111.6 \\
\hline 9 & & & & & & 4.3 & 36.9 & 34.9 & 3.3 & 31.0 & 106.6 \\
\hline 10 & & & & & & 4.2 & 37.7 & 33.4 & 3.2 & 31.4 & 101.9 \\
\hline 11 & & & & & & 4.1 & 37.8 & 32.5 & 3.0 & 31.5 & 95.4 \\
\hline 12 & & & & & & 4.3 & 38.7 & 33.3 & 3.3 & 32.0 & 103.0 \\
\hline 13 & & & & & & 4.9 & 40.0 & 36.7 & 3.4 & 32.5 & 104.6 \\
\hline 14 & & & & & & 4.7 & 40.5 & 34.8 & 3.3 & 33.0 & 100.1 \\
\hline
\end{tabular}

${ }^{\mathrm{a}}$ Grams of water consumed per animal per day.

${ }^{\mathrm{b}}$ Milligrams of $o$-chloropyridine consumed per kilogram body weight per day. 
Table G-4. Water and Compound Consumption by Female Mice in the Three-month Drinking Water Study of $o$-Chloropyridine

\begin{tabular}{|c|c|c|c|c|c|c|c|c|c|c|c|}
\hline \multirow[b]{2}{*}{ Week } & \multicolumn{2}{|c|}{0 ppm } & \multicolumn{3}{|c|}{10 ppm } & \multicolumn{3}{|c|}{30 ppm } & \multicolumn{3}{|c|}{$100 \mathrm{ppm}$} \\
\hline & $\begin{array}{l}\text { Watera } \\
\text { (g/day) }\end{array}$ & $\begin{array}{c}\text { Body } \\
\text { Weight (g) }\end{array}$ & $\begin{array}{l}\text { Water } \\
\text { (g/day) }\end{array}$ & $\begin{array}{c}\text { Body } \\
\text { Weight (g) }\end{array}$ & $\begin{array}{c}\text { Dose }^{b} \\
(\mathrm{mg} / \mathrm{kg})\end{array}$ & $\begin{array}{l}\text { Water } \\
\text { (g/day) }\end{array}$ & $\begin{array}{c}\text { Body } \\
\text { Weight (g) }\end{array}$ & $\begin{array}{c}\text { Dose } \\
(\mathrm{mg} / \mathrm{kg})\end{array}$ & $\begin{array}{l}\text { Water } \\
\text { (g/day) }\end{array}$ & $\begin{array}{c}\text { Body } \\
\text { Weight (g) }\end{array}$ & $\begin{array}{c}\text { Dose } \\
(\mathrm{mg} / \mathrm{kg})\end{array}$ \\
\hline 1 & 2.8 & 18.6 & 3.0 & 19.1 & 1.6 & 2.7 & 18.8 & 4.3 & 2.3 & 19.0 & 12.1 \\
\hline 2 & 3.1 & 19.9 & 2.9 & 20.6 & 1.4 & 2.9 & 20.0 & 4.3 & 2.7 & 19.7 & 13.7 \\
\hline 3 & 2.9 & 19.7 & 2.5 & 20.6 & 1.2 & 3.0 & 20.6 & 4.4 & 2.9 & 20.3 & 14.3 \\
\hline 4 & 3.3 & 21.4 & 2.6 & 19.5 & 1.3 & 2.5 & 21.4 & 3.5 & 2.8 & 21.5 & 13.0 \\
\hline 5 & 3.7 & 23.3 & 3.7 & 23.1 & 1.6 & 3.4 & 22.6 & 4.5 & 3.0 & 22.8 & 13.1 \\
\hline 6 & 3.0 & 23.2 & 3.1 & 23.3 & 1.3 & 3.0 & 23.8 & 3.8 & 2.5 & 23.0 & 10.9 \\
\hline 7 & 3.1 & 25.2 & 3.5 & 25.4 & 1.4 & 3.1 & 25.5 & 3.7 & 3.1 & 25.4 & 12.2 \\
\hline 8 & 2.8 & 25.6 & 3.1 & 26.5 & 1.2 & 2.8 & 26.1 & 3.2 & 2.5 & 26.3 & 9.5 \\
\hline 9 & 3.3 & 27.4 & 3.4 & 27.0 & 1.3 & 3.4 & 26.3 & 3.9 & 3.0 & 27.3 & 11.0 \\
\hline 10 & 3.1 & 27.0 & 3.2 & 27.7 & 1.2 & 3.3 & 27.7 & 3.6 & 3.1 & 27.8 & 11.1 \\
\hline 11 & 3.0 & 28.3 & 3.3 & 28.6 & 1.2 & 3.1 & 28.5 & 3.3 & 3.1 & 28.6 & 10.9 \\
\hline 12 & 3.7 & 29.3 & 3.7 & 29.2 & 1.3 & 3.2 & 29.5 & 3.3 & 3.1 & 29.7 & 10.4 \\
\hline 13 & 3.0 & 29.2 & 4.3 & 30.1 & 1.4 & 2.4 & 30.6 & 2.4 & 2.6 & 29.6 & 8.8 \\
\hline \multirow[t]{3}{*}{14} & 3.5 & 28.5 & 3.3 & 31.8 & 1.0 & 3.4 & 31.2 & 3.3 & 3.4 & 30.3 & 11.2 \\
\hline & & & & & & \multicolumn{3}{|c|}{300 ppm } & \multicolumn{3}{|c|}{1,000 ppm } \\
\hline & & & & & & $\begin{array}{l}\text { Water } \\
\text { (g/day) }\end{array}$ & $\begin{array}{c}\text { Body } \\
\text { Weight (g) }\end{array}$ & $\begin{array}{c}\text { Dose } \\
(\mathrm{mg} / \mathrm{kg})\end{array}$ & $\begin{array}{l}\text { Water } \\
\text { (g/day) }\end{array}$ & $\begin{array}{c}\text { Body } \\
\text { Weight (g) }\end{array}$ & $\begin{array}{c}\text { Dose } \\
(\mathrm{mg} / \mathrm{kg})\end{array}$ \\
\hline 1 & & & & & & 3.3 & 18.1 & 54.7 & 1.6 & 18.5 & 86.3 \\
\hline 2 & & & & & & 2.7 & 20.3 & 40.0 & 3.8 & 18.9 & 200.3 \\
\hline 3 & & & & & & 3.3 & 22.1 & 44.7 & 1.4 & 18.9 & 74.0 \\
\hline 4 & & & & & & 2.6 & 22.3 & 34.9 & 1.1 & 21.0 & 52.4 \\
\hline 5 & & & & & & 3.7 & 23.9 & 46.4 & 1.3 & 23.0 & 56.5 \\
\hline 6 & & & & & & 2.9 & 25.0 & 34.8 & 2.4 & 22.9 & 104.7 \\
\hline 7 & & & & & & 2.9 & 26.4 & 33.0 & 2.1 & 24.7 & 85.1 \\
\hline 8 & & & & & & 2.7 & 26.6 & 30.5 & 2.2 & 25.2 & 87.3 \\
\hline 9 & & & & & & 3.2 & 28.4 & 33.8 & 2.3 & 25.8 & 89.3 \\
\hline 10 & & & & & & 3.3 & 29.3 & 33.8 & 2.6 & 26.8 & 96.9 \\
\hline 11 & & & & & & 4.3 & 30.0 & 43.0 & 2.5 & 27.5 & 91.0 \\
\hline 12 & & & & & & 3.4 & 31.3 & 32.6 & 2.9 & 28.3 & 102.6 \\
\hline 13 & & & & & & 3.2 & 31.2 & 30.8 & 1.8 & 27.6 & 65.2 \\
\hline 14 & & & & & & 3.4 & 31.9 & 32.0 & 2.8 & 28.4 & 98.6 \\
\hline
\end{tabular}

${ }^{\mathrm{a}}$ Grams of water consumed per animal per day.

${ }^{\mathrm{b}}$ Milligrams of $o$-chloropyridine consumed per kilogram body weight per day. 


\section{Appendix H. Ingredients, Nutrient Composition, and Contaminant Levels in NTP-2000 Rat and Mouse Ration}

\section{Tables}

Table H-1. Ingredients of NTP-2000 Rat and Mouse Ration .....

H-2

Table H-2. Vitamins and Minerals in NTP-2000 Rat and Mouse Ration................................ H-3

Table H-3. Nutrient Composition of NTP-2000 Rat and Mouse Ration .................................. H-4

Table H-4. Contaminant Levels in NTP-2000 Rat and Mouse Ration .................................. H-6 
Table H-1. Ingredients of NTP-2000 Rat and Mouse Ration

\begin{tabular}{lc}
\hline \multicolumn{1}{c}{ Ingredients } & Percent by Weight \\
\hline Ground hard winter wheat & 22.26 \\
Ground \#2 yellow shelled corn & 22.18 \\
Wheat middlings & 15.0 \\
Oat hulls & 8.5 \\
Alfalfa meal (dehydrated, 17\% protein) & 7.5 \\
Purified cellulose & 5.5 \\
Soybean meal (49\% protein) & 5.0 \\
Fish meal (60\% protein) & 4.0 \\
Corn oil (without preservatives) & 3.0 \\
Soy oil (without preservatives) & 3.0 \\
Dried brewer's yeast & 1.0 \\
Calcium carbonate (USP) & 0.9 \\
Vitamin premix & 0.5 \\
Mineral premix & b \\
Calcium phosphate, dibasic (USP) & 0.5 \\
Sodium chloride & 0.4 \\
Choline chloride (70\% choline) & 0.3 \\
Methionine & 0.26 \\
\hline
\end{tabular}

${ }^{\mathrm{a}}$ Wheat middlings as carrier.

${ }^{\mathrm{b}}$ Calcium carbonate as carrier. 
$o$-Chloropyridine, NTP TOX 83

Table H-2. Vitamins and Minerals in NTP-2000 Rat and Mouse Ration ${ }^{\text {a }}$

\begin{tabular}{lcl}
\hline & Amount & \\
\hline Vitamins & & Source \\
A & $4,000 \mathrm{IU}$ & Stabilized vitamin A palmitate or acetate \\
$\mathrm{D}$ & $1,000 \mathrm{IU}$ & D-activated animal sterol \\
$\mathrm{K}$ & $1.0 \mathrm{mg}$ & Menadione sodium bisulfite complex \\
$\alpha$-Tocopheryl acetate & $100 \mathrm{IU}$ & - \\
Niacin & $23 \mathrm{mg}$ & - \\
Folic acid & $1.1 \mathrm{mg}$ & - \\
d-Pantothenic acid & $10 \mathrm{mg}$ & d-Calcium pantothenate \\
Riboflavin & $3.3 \mathrm{mg}$ & - \\
Thiamine & $4 \mathrm{mg}$ & Thiamine mononitrate \\
B12 & $5 \mu \mathrm{g}$ & - \\
Pyridoxine & $6.3 \mathrm{mg}$ & Pyridoxine hydrochloride \\
Biotin & $0.2 \mathrm{mg}$ & d-Biotin \\
\hline Minerals & & \\
Magnesium & $514 \mathrm{mg}$ & Magnesium oxide \\
Iron & $35 \mathrm{mg}$ & Iron sulfate \\
Zinc & $12 \mathrm{mg}$ & Zinc oxide \\
Manganese & $10 \mathrm{mg}$ & Manganese oxide \\
Copper & $2.0 \mathrm{mg}$ & Copper sulfate \\
Iodine & $0.2 \mathrm{mg}$ & Calcium iodate \\
Chromium & $0.2 \mathrm{mg}$ & Chromium acetate \\
\hline Per kg of finished product. & &
\end{tabular}


$o$-Chloropyridine, NTP TOX 83

Table H-3. Nutrient Composition of NTP-2000 Rat and Mouse Ration

\begin{tabular}{|c|c|c|c|}
\hline Nutrient & $\begin{array}{c}\text { Mean } \pm \text { Standard } \\
\text { Deviation }\end{array}$ & Range & Number of Samples \\
\hline Protein (\% by weight) & 15.2 & 15.2 & 1 \\
\hline Crude fat ( $\%$ by weight) & 8.0 & 8.0 & 1 \\
\hline Crude fiber ( $\%$ by weight) & 943 & 9.4 & 1 \\
\hline Ash (\% by weight) & 5.3 & 5.3 & 1 \\
\hline \multicolumn{4}{|c|}{ Amino Acids (\% of total diet) } \\
\hline Arginine & $0.783 \pm 0.070$ & $0.670-0.970$ & 22 \\
\hline Cystine & $0.220 \pm 0.024$ & $0.150-0.250$ & 22 \\
\hline Glycine & $0.701 \pm 0.041$ & $0.620-0.800$ & 22 \\
\hline Histidine & $0.352 \pm 0.077$ & $0.270-0.680$ & 22 \\
\hline Isoleucine & $0.546 \pm 0.044$ & $0.430-0.660$ & 22 \\
\hline Leucine & $1.095 \pm 0.067$ & $0.960-1.240$ & 22 \\
\hline Lysine & $0.711 \pm 0.114$ & $0.310-0.860$ & 22 \\
\hline Methionine & $0.409 \pm 0.046$ & $0.260-0.490$ & 22 \\
\hline Phenylalanine & $0.628 \pm 0.040$ & $0.540-0.720$ & 22 \\
\hline Threonine & $0.505 \pm 0.043$ & $0.430-0.610$ & 22 \\
\hline Tryptophan & $0.150 \pm 0.028$ & $0.110-0.200$ & 22 \\
\hline Tyrosine & $0.401 \pm 0.061$ & $0.280-0.540$ & 22 \\
\hline Valine & $0.665 \pm 0.043$ & $0.550-0.730$ & 22 \\
\hline \multicolumn{4}{|c|}{ Essential Fatty Acids (\% of total diet) } \\
\hline Linoleic & $3.95 \pm 0.259$ & $3.49-4.55$ & 22 \\
\hline Linolenic & $0.30 \pm 0.032$ & $0.21-0.35$ & 22 \\
\hline \multicolumn{4}{|l|}{ Vitamins } \\
\hline Vitamin A (IU/kg) & 6,920 & 6,920 & 1 \\
\hline Vitamin D (IU/kg) & $1,000^{\mathrm{a}}$ & - & - \\
\hline$\alpha$-Tocopherol (ppm) & $80.6 \pm 22.03$ & $27.0-124.0$ & 22 \\
\hline Thiamine (ppm) $)^{\mathrm{b}}$ & 8.8 & 8.8 & 1 \\
\hline Riboflavin (ppm) & $7.6 \pm 2.89$ & $4.20-17.50$ & 22 \\
\hline Niacin (ppm) & $78.9 \pm 9.08$ & $66.4-98.2$ & 22 \\
\hline Pantothenic acid (ppm) & $26.9 \pm 12.63$ & $17.4-81.0$ & 22 \\
\hline Pyridoxine $(\mathrm{ppm})^{\mathrm{b}}$ & $9.54 \pm 1.99$ & $6.44-13.7$ & 22 \\
\hline Folic acid (ppm) & $1.62 \pm 0.48$ & $1.15-3.27$ & 22 \\
\hline Biotin (ppm) & $0.32 \pm 0.10$ & $0.20-0.704$ & 22 \\
\hline Vitamin $B_{12}(\mathrm{ppb})$ & $53.6 \pm 39.6$ & $18.3-174.0$ & 22 \\
\hline Choline $(\mathrm{ppm})^{\mathrm{b}}$ & $2,846 \pm 485$ & $1,820-3,790$ & 22 \\
\hline
\end{tabular}


$o$-Chloropyridine, NTP TOX 83

\begin{tabular}{lccc}
\hline \multicolumn{1}{c}{ Nutrient } & $\begin{array}{c}\text { Mean } \pm \text { Standard } \\
\text { Deviation }\end{array}$ & Range & Number of Samples \\
\hline Minerals & & & 1 \\
Calcium (\%) & 0.959 & 0.959 & 1 \\
Phosphorus (\%) & 0.589 & 0.589 & 22 \\
Potassium (\%) & $0.666 \pm 0.030$ & $0.626-0.733$ & 22 \\
Chloride (\%) & $0.386 \pm 0.039$ & $0.300-0.474$ & 22 \\
Sodium (\%) & $0.189 \pm 0.016$ & $0.160-0.222$ & 22 \\
Magnesium (\%) & $0.216 \pm 0.062$ & $0.185-0.490$ & 14 \\
Sulfur (\%) & $0.170 \pm 0.029$ & $0.116-0.209$ & 22 \\
Iron (ppm) & $186 \pm 39.2$ & $135-311$ & 22 \\
Manganese (ppm) & $51.4 \pm 10.28$ & $21.0-73.1$ & 22 \\
Zinc (ppm) & $53.4 \pm 8.46$ & $43.3-78.5$ & 22 \\
Copper (ppm) & $7.01 \pm 2.562$ & $3.21-16.3$ & 22 \\
Iodine (ppm) & $0.503 \pm 0.206$ & $0.158-0.972$ & 22 \\
Chromium (ppm) & $0.694 \pm 0.276$ & $0.330-1.380$ & 22 \\
Cobalt (ppm) & $0.256 \pm 0.164$ & $0.098-0.864$ & \\
\hline
\end{tabular}

aFrom formulation.

${ }^{\mathrm{b}}$ As hydrochloride (thiamine and pyridoxine) or chloride (choline). 
$o$-Chloropyridine, NTP TOX 83

Table H-4. Contaminant Levels in NTP-2000 Rat and Mouse Ration ${ }^{\text {a }}$

\begin{tabular}{|c|c|c|c|}
\hline & Mean $^{b}$ & Range & Number of Samples \\
\hline \multicolumn{4}{|l|}{ Contaminants } \\
\hline Arsenic (ppm) & 0.50 & 0.50 & 1 \\
\hline Cadmium (ppm) & 0.08 & 0.08 & 1 \\
\hline Lead (ppm) & 0.05 & 0.05 & 1 \\
\hline Mercury (ppm) & $<0.02$ & - & 1 \\
\hline Selenium (ppm) & 0.16 & 016 & 1 \\
\hline Aflatoxins (ppb) & $<5.00$ & - & 1 \\
\hline Nitrate nitrogen $(\mathrm{ppm})^{\mathrm{c}}$ & 17.7 & 17.7 & 1 \\
\hline Nitrite nitrogen $(\mathrm{ppm})^{\mathrm{c}}$ & $<0.61$ & - & 1 \\
\hline BHA $(\mathrm{ppm})^{\mathrm{d}}$ & $<1.0$ & - & 1 \\
\hline BHT $(\mathrm{ppm})^{\mathrm{d}}$ & $<1.0$ & - & 1 \\
\hline Aerobic plate count (CFU/g) & 10 & 10 & 1 \\
\hline Coliform (MPN/g) & 3.0 & 3.0 & 1 \\
\hline Escherichia coli (MPN/g) & $<10$ & - & 1 \\
\hline Salmonella (MPN/g) & Negative & - & 1 \\
\hline Total nitrosoamines $(\mathrm{ppb})^{\mathrm{e}}$ & 3.5 & 3.5 & 1 \\
\hline$N$-Nitrosodimethylamine $(\mathrm{ppb})^{\mathrm{e}}$ & 1.8 & 1.8 & 1 \\
\hline$N$-Nitrosopyrrolidine $(\mathrm{ppb})^{\mathrm{e}}$ & 1.7 & 1.7 & 1 \\
\hline \multicolumn{4}{|l|}{ Pesticides (ppm) } \\
\hline$\alpha-\mathrm{BHC}$ & $<0.01$ & - & 1 \\
\hline$\beta-\mathrm{BHC}$ & $<0.02$ & - & 1 \\
\hline$\gamma$-BHC & $<0.01$ & - & 1 \\
\hline$\delta$-BHC & $<0.01$ & - & 1 \\
\hline Heptachlor & $<0.01$ & - & 1 \\
\hline Aldrin & $<0.01$ & - & 1 \\
\hline Heptachlor epoxide & $<0.01$ & - & \\
\hline DDE & $<0.01$ & - & 1 \\
\hline DDD & $<0.01$ & - & 1 \\
\hline DDT & $<0.01$ & - & 1 \\
\hline $\mathrm{HCB}$ & $<0.01$ & - & 1 \\
\hline Mirex & $<0.01$ & - & 1 \\
\hline Methoxychlor & $<0.05$ & - & 1 \\
\hline Dieldrin & $<0.01$ & - & 1 \\
\hline Endrin & $<0.01$ & - & 1 \\
\hline Telodrin & $<0.01$ & - & 1 \\
\hline
\end{tabular}


$o$-Chloropyridine, NTP TOX 83

\begin{tabular}{lccc}
\hline & Mean $^{\mathbf{b}}$ & Range & Number of Samples \\
\hline Chlordane & $<0.05$ & - & 1 \\
Toxaphene & $<0.10$ & - & 1 \\
Estimated PCBs & $<0.20$ & - & 1 \\
Ronnel & $<0.01$ & - & 1 \\
Ethion & $<0.02$ & - & 1 \\
Trithion & $<0.05$ & - & 1 \\
Diazinon & $<0.10$ & - & 1 \\
Methyl chlorpyrifos & 0.175 & 0.175 & 1 \\
Methyl parathion & $<0.02$ & - & 1 \\
Ethyl parathion & $<0.02$ & - & 1 \\
Malathion & 0.589 & 0.589 & 1 \\
Endosulfan I & $<0.01$ & - & 1 \\
Endosulfan II & $<0.01$ & - & 1 \\
Endosulfan sulfate & $<0.03$ & - & 1 \\
\hline
\end{tabular}

${ }^{\mathrm{a}}$ All samples were irradiated. $\mathrm{CFU}=$ colony-forming units; MPN $=$ most probable number; $\mathrm{BHC}=$ hexachlorocyclohexane or benzene hexachloride.

${ }^{b}$ For values less than the limit of detection, the detection limit is given as the mean.

'Sources of contamination: alfalfa, grains, and fish meal.

${ }^{\mathrm{d} S}$ Sources of contamination: soy oil and fish meal.

${ }^{\mathrm{e}} \mathrm{All}$ values were corrected for percent recovery. 
o-Chloropyridine, NTP TOX 83

\section{Appendix I. Sentinel Animal Program \\ Table of Contents}

I.1. Methods

$\mathrm{I}-2$

I.2. Results

I-2

\section{Tables}

Table I-1. Laboratory Methods and Agents Tested for in the Sentinel Animal Program............. I-2 


\section{I.1. Methods}

Rodents used in the National Toxicology Program are produced in optimally clean facilities to eliminate potential pathogens that may affect study results. The Sentinel Animal Program is part of the periodic monitoring of animal health that occurs during the toxicologic evaluation of test compounds. Under this program, the disease state of the rodents is monitored via sera from extra (sentinel) or dosed animals in the study rooms. The sentinel animals and the dosed animals are subject to identical environmental conditions. Furthermore, the sentinel animals come from the same production source and weanling groups as the animals used for the studies of test compounds.

For the 3-month studies, blood samples were collected, allowed to clot, and the serum was separated. The serum samples were processed appropriately at BioReliance Corporation (Rockville, MD) for determination of the presence of pathogens. The laboratory methods and agents for which testing was performed are tabulated below; the times at which samples were collected during the studies are also listed.

Table I-1. Laboratory Methods and Agents Tested for in the Sentinel Animal Program

\begin{tabular}{ll}
\hline \multicolumn{1}{c}{ Method and Test } & Time of Collection \\
\hline Rats & \\
ELISA & \\
PVM (pneumonia virus of mice) & Study termination \\
RCV/SDA (rat coronavirus/sialodacryoadenitis virus) & Study termination \\
Sendai & Study termination \\
Immunofluorescence Assay & \\
Parvovirus & Study termination \\
Mice & \\
ELISA & \\
Ectromelia virus & \\
EDIM (epizootic diarrhea of infant mice) & Study termination \\
GDVII (mouse encephalomyelitis virus) & Study termination \\
LCM (lymphocytic choriomeningitis virus) & Study termination \\
MAd-FL (Mouse adenoma virus-)1 & Study termination \\
MHV (mouse hepatitis virus) & Study termination \\
PVM & Study termination \\
Reovirus & Study termination \\
Sendai & Study termination \\
Immunofluorescence Assay & Study termination \\
Parvovirus & \\
\hline
\end{tabular}

\section{I.2. Results}

All results were negative. 


\section{Appendix J. Absorption, Distribution, Metabolism, Excretion, and Toxicokinetics}

\section{Table of Contents}

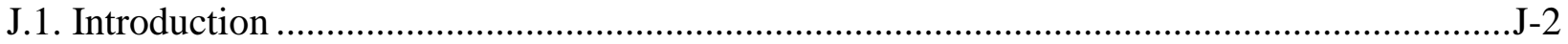

J.2. Materials and Methods .............................................................................................

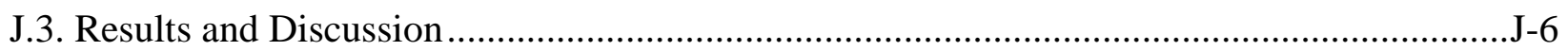

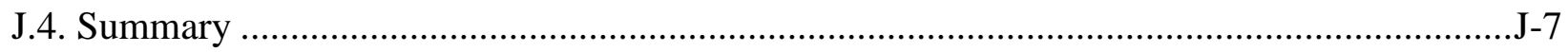

\section{Tables}

Table J-1. Disposition of Radioactivity in Male F344 Rats 72 Hours Following a Single

Oral Gavage Dose of $10 \mathrm{mg} / \mathrm{kg}\left[{ }^{14} \mathrm{C}\right]-o$-Chloropyridine.

Table J-2. Tissue Distribution of Radioactivity in Male F344 Rats 72 Hours Following a Single Oral Gavage Dose of $10 \mathrm{mg} / \mathrm{kg}\left[{ }^{14} \mathrm{C}\right]-o$-Chloropyridine.

Table J-3. Disposition of Radioactivity in Male F344 Rats 72 Hours Following a Single Oral Gavage Dose of $0.1 \mathrm{mg} / \mathrm{kg}\left[{ }^{14} \mathrm{C}\right]-o$-Chloropyridine.

Table J-4. Tissue Distribution of Radioactivity in Male F344 Rats 72 Hours Following a Single Oral Gavage Dose of $0.1 \mathrm{mg} / \mathrm{kg}\left[{ }^{14} \mathrm{C}\right]-o$-Chloropyridine...

Table J-5. Excretion of Radioactivity in Bile in Male F344 Rats Following a Single Intraperitoneal Injection of $50 \mathrm{mg} / \mathrm{kg}\left[{ }^{14} \mathrm{C}\right]-o$-Chloropyridine

Table J-6. Total Radioactive Equivalents in Blood Over Time in Male F344 Rats Following a Single Oral Gavage Dose of $10 \mathrm{mg} / \mathrm{kg}\left[{ }^{14} \mathrm{C}\right]-o$-Chloropyridine.....

Table J-7. Concentration of $o$-Chloropyridine and Total Radioactive Equivalents in Blood Over Time in Male F344 Rats Following a Single Intravenous Injection of $1 \mathrm{mg} / \mathrm{kg}\left[{ }^{14} \mathrm{C}\right]$-o-Chloropyridine.

\section{Figures}

Figure J-1. $\left[{ }^{14} \mathrm{C}\right]$-Labeled Urinary Metabolites in Male F344 Rats Following a Single Oral Gavage Dose of $10 \mathrm{mg} / \mathrm{kg}\left[{ }^{14} \mathrm{C}\right]-o$-Chloropyridine

Figure J-2. Disubstituted Urinary Metabolites of $o$-Chloropyridine in Male F344 Rats $\mathrm{J}-16$

Figure J-3. Blood Timecourse and Toxicokinetic Parameter Estimates of $o$ Chloropyridine in Male F344 Rats Following a Single Oral Gavage Dose of $10 \mathrm{mg} / \mathrm{kg}\left[{ }^{14} \mathrm{C}\right]-o$-Chloropyridine

Figure J-4. Blood Timecourse and Toxicokinetic Parameter Estimates for $o$ Chloropyridine and Radiolabel (o-Chloropyridine Equivalents) in Blood of Male F344 Rats Following a Single Intravenous Injection of $1 \mathrm{mg} / \mathrm{kg}$ $\left[{ }^{14} \mathrm{C}\right]-o$-Chloropyridine 


\section{J.1. Introduction}

The objective of these studies was to determine the disposition and metabolism of $o$-chloropyridine in male F344 rats after a single oral gavage administration of 0.1 or $10 \mathrm{mg} / \mathrm{kg}$ of $o$-chloropyridine. Limited studies were also conducted following intravenous and intraperitoneal dosing. Concentration of parent compound or the total radioactivity was also determined in blood in order to establish basic toxicokinetic parameters. Attempts were also made to identify metabolites of $o$-chloropyridine in rat urine and bile after a single oral or intraperitoneal dose.

\section{J.2. Materials and Methods}

\section{J.2.1. Chemicals}

Nonradiolabeled $o$-chloropyridine (CAS No. 109-09-1, purity 99\%, lot 03721) was purchased from Aldrich Chemical Company, Inc. (Milwaukee, WI). The identity of nonradiolabeled $o$-chloropyridine was confirmed by mass spectrometry (MS). The position of the chloro substituent was confirmed by chromatographic resolution of isomeric mono- and dichloropyridine standards using high-performance liquid chromatography (HPLC).

$o$-Chloropyridine radiolabeled with ${ }^{14} \mathrm{C}$ in the 2 and 6 positions (lot 3228-292, specific activity $58.50 \mathrm{mCi} / \mathrm{mmol}$ ), in an ethanol solution was received from New England Nuclear Corporation (Boston, MA).

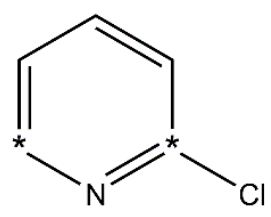

Structure of $\left[{ }^{14} \mathrm{C}\right]-o$-chloropyridine

$\left(*\right.$ denotes position of ${ }^{14} \mathrm{C}$ )

The radiochemical purity of the test material was determined to be equal to or higher than $95 \%$ by HPLC.

Prospective metabolites of $o$-chloropyridine, N-methylpyridine-2-mercapturic acid, pyridine-Noxide-2-mercapturic acid, 2-methylthiopyridine, 2-methylthiopyridine-N-oxide, 2-chloro-5pyridinol, and 2-chloro-4-pyridinol were synthesized in-house using methods of Talik ${ }^{58}$, Beak and Lee $^{59}$, Westland et al. ${ }^{60}$, and Kohrman et al. ${ }^{61}$.

\section{J.2.2. Animals}

Young adult male F344 rats were obtained from Charles River Laboratories, Inc. (Raleigh, NC), and were quarantined for at least 1 week prior to the start of the study. Animals were fed certified Purina Rodent Chow (5002; Purina Mills, Inc., St. Louis, MO) and furnished tap water ad libitum. During acclimation (1 day prior to dosing) and following dosing, metabolism study animals were housed individually in metabolism chambers that provided for separate collection of urine, feces, and volatiles. Biliary excretion study rats were kept on heating pads to maintain body temperature throughout the study. Toxicokinetic study rats were housed individually in 
cages following surgical implantation of the jugular cannulae and throughout the toxicokinetic study. In the animal rooms, air circulation was $100 \%$ fresh filtered, room temperature was maintained at $64^{\circ}$ to $79^{\circ} \mathrm{F}$, and relative humidity ranged from $30 \%$ to $70 \%$. Light and darkness were cycled at 12-hour intervals.

Anesthesia was used to avoid undue pain or distress. Rats were anesthetized with ketamine/xylazine $(7: 1,50$ to $80 \mathrm{mg} / \mathrm{kg}$ intramuscular or to effect) prior to surgical implantation of the jugular cannula ${ }^{62}$ for toxicokinetic studies. Biliary excretion study rats were anesthetized with sodium pentobarbital, orally ( $35 \mathrm{mg} / \mathrm{kg}$ ) and intraperitoneally ( $45 \mathrm{mg} / \mathrm{kg}$ ), prior to cannulation of the bile duct. A state of anesthesia was maintained throughout the bile collection period by injection of sodium pentobarbital $(5 \mathrm{mg} / \mathrm{kg})$ as needed. At the end of the oral gavage studies, rats were euthanized by exsanguination and section of the diaphragm. Toxicokinetic and biliary excretion study rats were euthanized by $\mathrm{CO}_{2}$ overexposure after the final collection.

\section{J.2.3. Study Design}

Absorption, distribution, metabolism, and excretion of $o$-chloropyridine were investigated in groups of rats following a single oral gavage dose of either 0.1 or $10 \mathrm{mg}\left[{ }^{14} \mathrm{C}\right]-$ $o$-chloropyridine/kg body weight $(10 \mu \mathrm{Ci}$ per animal) and killed 72 hours following dosing. Toxicokinetics of $o$-chloropyridine was investigated in jugular vein-cannulated rats receiving a single oral gavage dose of $10 \mathrm{mg} / \mathrm{kg}$ or a single intravenous injection of $1 \mathrm{mg} / \mathrm{kg}(30 \mu \mathrm{Ci}$ per animal in each case). Biliary excretion and metabolism were investigated in rats receiving a single intraperitoneal injection of $50 \mathrm{mg} / \mathrm{kg}$ ( $35 \mu \mathrm{Ci}$ per animal) for bile collection for up to 4 hours.

For all studies, formulations were prepared by addition of Alkamuls ${ }^{\circledR}$ EL-620/L (no more than $15 \%$ of the dose) (Rhodia, Inc., Cranbury, NJ) to known amounts of $o$-chloropyridine following which distilled deionized water and the appropriate amount of $\left[{ }^{14} \mathrm{C}\right]-O$-chloropyridine were added. Dosing volumes were either $5 \mathrm{~mL} / \mathrm{kg}$ (oral gavage and intraperitoneal injection or $1 \mathrm{~mL} / \mathrm{kg}$ (intravenous injection). Oral doses were administered by intragastic gavage using a syringe equipped with a 16-gauge gavage needle. Intravenous doses were injected into a lateral tail vein using a syringe equipped with a 27 -gauge needle.

\section{J.2.4. Sample Collection}

In mass balance studies, urine and feces were collected separately into containers cooled with dry ice for up to 72 hours. At terminal kill, urine was collected directly from the bladder and added to the final urine collection. After terminal kill, the cages were rinsed well with water and ethanol. Urine, feces, and cage rinse collections were stored in the dark at $-20^{\circ} \mathrm{C}$ until analyzed. Volatiles were collected by passing air from the metabolism chamber through a series of traps containing ethanol and $1 \mathrm{~N} \mathrm{NaOH}$ for collection of volatile organics and $\mathrm{CO}_{2}$, respectively.

At terminal kill, blood from anesthetized rats was collected into a heparinized syringe by cardiac puncture. Duplicate samples of skin (ears), triplicate samples of muscle (hind leg and abdominal) and adipose tissue (perirenal and epididymal), and the entire kidneys, liver, spleen, lungs, testes, bladder, heart, and brain were removed. The stomach, small intestine, cecum, and large intestine (all included contents) were removed and the carcasses were stored. 
Bile was collected via a bile duct cannula extension for up to 4 hours postdosing. Bile collections were stored at $-80^{\circ} \mathrm{C}$ prior to analysis.

In toxicokinetic studies, blood samples were collected from rats via a jugular cannula for up to 72 hours postdosing (oral gavage study) and for up to 4 hours postdosing (intravenous injection study). In the rat intravenous injection study, the volume of blood withdrawn at each time point was replaced with plasma obtained from donor animals. Blood samples in these studies were extracted immediately after collection as described below.

\section{J.2.5. Sample Analysis}

Whole blood samples (approximately $300 \mu \mathrm{L}$ per animal per time point) from toxicokinetic studies were extracted three times with twice the volume of hexanes. The three extracts from each sample were pooled and stored at $-20^{\circ} \mathrm{C}$ prior to analysis for total radioactivity and for parent $o$-chloropyridine (intravenous studies only) by HPLC as described below.

Radioactivity in samples was measured by liquid scintillation spectrophotometry (LSS). Ultima Gold $^{\mathrm{TM}}$ scintillation cocktail (Packard Instrument Company, Meriden, CT) was used in all determinations of radiochemical content. Duplicate aliquots of urine, duplicate aliquots of bile, triplicate aliquots of cage rinse and trapping solutions from breath traps, and duplicate aliquots of blood extracts were directly analyzed. Triplicate samples of feces were weighed, homogenized with equal amounts of water, and solubilized in Soluene ${ }^{\circledR}-350$ (Packard Instrument Company). Tissue samples (from large tissues) and small tissues and organs in their entirety were digested in Soluene ${ }^{\circledR}-350$. Large organs like the liver were homogenized, and weighed aliquots of the homogenate were used. Blood samples were neutralized and decolorized by treatment with perchloric acid and $\mathrm{H}_{2} \mathrm{O}_{2}$ prior to solubilization. Gastrointestinal tract tissues and carcass were digested in $2 \mathrm{~N}$ ethanolic $\mathrm{NaOH}$ prior to analysis of triplicate aliquots for radioactivity.

\section{J.2.6. Metabolic Profiling and Identification}

Aliquots of the 1-hour and 4-hour bile collections were mixed with an equal amount of the initial-condition mobile phase containing $0.1 \%$ trifluoroacetic acid (TFA) and the mixture was centrifuged prior to analysis by HPLC (see below). An aliquot of bile was also incubated with $\beta$ glucuronidase type VII-A, obtained from Escherichia coli for 17 hours at $37^{\circ} \mathrm{C}$, and centrifuged prior to HPLC analysis.

Composite urine samples ( 0 to 24 hours) from the $10 \mathrm{mg} / \mathrm{kg}$ oral gavage study were profiled using HPLC. Urine was subjected to acid hydrolysis to determine whether acid labile conjugates were present. Urine was hydrolyzed by addition of $1 \mathrm{~N} \mathrm{HCl}$ followed by heating overnight between $50^{\circ}$ and $55^{\circ} \mathrm{C}$. After hydrolysis, the samples were neutralized and analyzed by HPLC/LSS.

Radiolabeled urinary metabolites were isolated using HPLC as described below. Fractions containing the same metabolites collected from a number of injections were pooled and concentrated. Other chromatographic conditions were then developed for the separation of the urinary metabolites in the fractions containing multiple radiolabeled components. Selected fractions were analyzed by MS and nuclear magnetic resonance (NMR) spectroscopy for identification of metabolites. 


\section{J.2.7. High-Performance Liquid Chromatography}

The HPLC system consisted of two Waters pumps (models 515, 510, or 6000A; Waters Corporation, Milford, MA) or a Waters 600E solvent delivery system, a Rheodyne Model 7125 injector (Rheodyne, LLC, Rohnert Park, CA), an ABI 759A absorbance detector (set at 230 or 254 nm; Applied Biosystems, Inc., Foster City, CA) or a Waters 2487 dual absorbance detector (set at 250 and $285 \mathrm{~nm}$ ), and a $\beta$ RAM-LS radioactivity detector equipped with a 200-, 250-, or $500-\mu \mathrm{L}$ lithium glass flowthrough solid scintillator flow cell. The flow rate was $1 \mathrm{~mL} / \mathrm{minute}$ unless otherwise specified. Column effluent was collected in timed fractions and assayed for $\left[{ }^{14} \mathrm{C}\right]$ content using LSS.

Blood extracts for $o$-chloropyridine levels were analyzed on an Alltech ${ }^{\circledR}$ Alltima ${ }^{\mathrm{TM}}$ Cyano $100-\AA$ column $(250 \mathrm{~mm} \times 4.6 \mathrm{~mm}, 5 \mu \mathrm{m}$ particle size; Alltech Associates, Inc., Deerfield, IL). The analysis was isocratic with a mobile phase consisting of 95:5 (v:v) hexanes:ethanol.

Profiling of urinary metabolites was done on a Phenomenex ${ }^{\circledR}$ Luna ${ }^{\circledR} \mathrm{C} 18$ column $(150 \mathrm{~mm} \times 4.6 \mathrm{~mm}, 5 \mu \mathrm{m}$ particle size; Phenomenex, Inc., Torrance, CA) using acetonitrile and water, both containing $0.1 \%$ TFA. The acetonitrile gradient was linearly increased as follows: $2 \%$ for 5 minutes, to $10 \%$ over 15 minutes, and then to $50 \%$ over 5 minutes. Acid-hydrolyzed urine samples were analyzed the same way except that the gradient was changed from $2 \%$ to $20 \%$ over 15 minutes.

Further profiling of the urinary metabolites and profiling of bile utilized a Phenomenex ${ }^{\circledR}$ Aqua $^{\circledR}$ C18 column $(250 \mathrm{~mm} \times 4.6 \mathrm{~mm}, 5 \mu \mathrm{m}$ particle size $)$; the mobile phases used were $100 \% 0.05 \mathrm{M}$ ammonium acetate buffer, $\mathrm{pH} 4$, and acetonitrile. Urine was eluted with a linear gradient from $0 \%$ to $75 \%$ acetonitrile over 30 minutes. Bile was eluted with water and acetonitrile, both containing $0.1 \%$ TFA, at a flow rate of $2 \mathrm{~mL} /$ minute. The acetonitrile gradient was linearly increased as follows: $3 \%$ for 2 minutes, to $11 \%$ over 16 minutes, to $25 \%$ over 6 minutes, and then to $90 \%$ over 2 minutes. For the isolation of biliary metabolites, the same mobile phase gradient was run at a flow rate of $4 \mathrm{~mL} /$ minute on a Phenomenex ${ }^{\circledR}$ Aqua $^{\circledR} \mathrm{C} 18$ column $(250 \mathrm{~mm} \times 10 \mathrm{~mm}, 5 \mu \mathrm{m}$ particle size $)$.

Urinary metabolites were isolated using a Phenomenex ${ }^{\circledR}$ Aqua ${ }^{\circledR} \mathrm{C} 18$ column $(250 \mathrm{~mm} \times 10 \mathrm{~mm}$, $5 \mu \mathrm{m}$ particle size) using acetonitrile and water, both containing $0.1 \%$ TFA. The acetonitrile gradient was linearly increased as follows: $3 \%$ for 5 minutes, to $10 \%$ over 20 minutes, to $15 \%$ over 5 minutes, and then to $90 \%$ over 2 minutes. The flow rate was $4 \mathrm{~mL} /$ minute. Variations of this method using isocratic conditions of $8 \%, 10 \%, 20 \%$, or $25 \%$ acetonitrile were used to further purify the urine fractions as necessary.

Analysis of urinary fractions for metabolite identification by LC/MS used a Phenomenex ${ }^{\circledR}$ Aqua ${ }^{\circledR} \mathrm{C} 18$ column $(250 \mathrm{~mm} \times 4.6 \mathrm{~mm}, 5 \mu \mathrm{m}$ particle size $)$. The mobile phases used were acetonitrile and water, both containing $0.1 \%$ TFA at isocratic conditions of $20 \%, 30 \%, 40 \%$, or $60 \%$ acetonitrile and a flow rate of $0.5 \mathrm{~mL} / \mathrm{minute}$.

\section{J.2.8. Other Instrumentation}

NMR spectra were obtained using a Bruker ${ }^{\circledR}$ AMX-500 NMR spectrometer (Bruker BioSpin Corporation, Newark, DE) and a Bruker ${ }^{\circledR}$ AVANCE $^{\mathrm{TM}}-300$ spectrometer. Mass spectra were obtained using a Hewlett Packard 6890 Series capillary gas chromatograph (Hewlett-Packard 
Company, Palo Alto, CA) and a HP 5973 mass selective detector or on a PE SCIEX API 150 EX analyzer (Perkin Elmer Sciex, Wellesley, MA) attached to an Agilent 110 Series Tower (Agilent Technologies) with an IN/US PRAM (IN/US Systems, Inc., Tampa, FL) inline.

\section{J.2.9. Toxicokinetic Analysis}

Blood concentration-time data were analyzed by model-dependent methods using WinNonlin ${ }^{\circledR}$ version 1.0 (Pharsight Corporation, Mountain View, CA). Three different methods of weighting the data points (uniform, 1/y, and $1 / \mathrm{y}^{2}$ ) were utilized. A statistical $\mathrm{F}$ test was used for the selection of the appropriate number of compartments for the best-fit model. The twocompartment model resulted in the best fits to the blood $o$-chloropyridine concentration-time data in rats after intravenous injection. The data for one rat were poorly fitted by the simulation at the last two time points. For this reason, this rat was excluded from the analysis. The twocompartment model is described by the following equation:

$$
C(t)=A \times e^{-\alpha t}+B \times e^{-\beta t}
$$

where constants A and B are intercepts on the $\mathrm{y}$ axis for each exponential segment of the curve and $\beta$ and $\alpha$ are the elimination and distribution rate constants, respectively.

\section{J.3. Results and Discussion}

\section{J.3.1. Metabolism and Disposition of $o$-Chloropyridine}

Disposition data for rats receiving a single oral gavage dose of $10 \mathrm{mg}\left[{ }^{14} \mathrm{C}\right]-O$-chloropyridine/kg body weight are presented in Table J-1. As shown in the table, $81.9 \%$ of the dose was recovered after 72 hours with $35.9 \%$ in the urine, $28.4 \%$ in the feces, and $12.5 \%$ and $1.14 \%$, respectively, as $\mathrm{CO}_{2}$ and as volatile organics. Tissue distribution of radiolabel in the same animals is shown in Table J-2. Approximately $0.5 \%$ and $3.4 \%$ of the dose was found in the residual carcass and selected tissues, respectively, at 72 hours after dosing. The liver and kidney were the only tissues that were found to have appreciable tissue:blood ratios. Data for rats receiving a single oral gavage dose of $0.1 \mathrm{mg} / \mathrm{kg}\left[{ }^{14} \mathrm{C}\right]-o$-chloropyridine show that there are no dose-related differences in the excretion pattern; approximately $38.3 \%, 28 \%, 10.5 \%$, and less than $1 \%$ was excreted in urine, in feces, as $\mathrm{CO}_{2}$, and as volatile organics, respectively (Table $\mathrm{J}-3$ and Table $\mathrm{J}-4$ ).

The biliary excretion study conducted in rats following a single intraperitoneal injection of $50 \mathrm{mg} / \mathrm{kg}\left[{ }^{14} \mathrm{C}\right]$-o-chloropyridine showed that approximately $27 \%$ of the dose was excreted in bile within 4 hours after dosing (Table J-5). Because only $8 \%$ of a $50 \mathrm{mg} / \mathrm{kg}$ intaperitoneal dose of $\left[{ }^{14} \mathrm{C}\right]-O$-chloropyridine was excreted in feces within 24 hours in a pilot study (data not shown), a significant recirculation of the dose from the lower gastrointestinal tract can be expected.

A profile of urine from oral gavage administration is shown in Figure $\mathrm{J}-1$. At least nine peaks were detected of which several were not retained by reverse phase chromatography, but did not cleave by acid hydrolysis. Less than $1 \%$ of the recovered ${ }^{14} \mathrm{C}$ eluted at the retention time for the parent compound suggesting significant metabolism of $o$-chloropyridine following oral gavage administration. Based on the metabolism of other pyridines ${ }^{63-65}$, the urinary excretion of several metabolites was anticipated. Metabolism may include $\mathrm{C}$ - and $\mathrm{N}$-oxidation with some conjugation possible. N-Methylation is a significant route of metabolism of pyridine compounds in certain species, including the rat ${ }^{66}$. 
Urinary metabolite fractions were isolated using semipreparative HPLC for further characterization. Analyses of three major fractions by NMR spectroscopy and MS indicated disubstituted pyridine structures. Data from a proton NMR spectrum substantiated the structure for one metabolite to contain a mercapturic acid as one substituent and a hydroxyl group as the other substituent on the pyridine ring (Figure J-2). However, it was uncertain whether the hydroxyl group was located at the 4 or 5 position on the pyridine ring. Mass spectral and proton NMR data indicated the structures for two other metabolites to be consistent with hydroxylated $o$-chloropyridines. One of these was confirmed to be 2-chloro-5-hydroxypyridine by comparison with a synthetic standard (Figure J-2). For the other metabolite, the position of the hydroxyl substitution could not be confirmed (Figure J-2). The HPLC retention time and proton NMR spectra of the third metabolite did not match with those of a synthetic standard of 2-chloro4-pyridinol (Figure J-2).

Profiling bile after a single intraperitoneal injection of $50 \mathrm{mg} / \mathrm{kg}\left[{ }^{14} \mathrm{C}\right]-\mathrm{o}$-chloropyridine showed that the excretion of metabolites in bile was less complex than in urine following oral exposure potentially due to route and/or matrix differences. Incubation of the chromatographically separated bile fractions with $\beta$-glucuronidase obtained from $E$. coli showed shifts in six of the eight metabolite fractions analyzed, indicating the presence of several glucuronide metabolites (results not shown).

\section{J.3.2. Toxicokinetics}

$o$-Chloropyridine radioactive equivalents in blood (ng-equivalents/g blood) over time in male rats given a single gavage dose of $10 \mathrm{mg}\left[{ }^{14} \mathrm{C}\right]-o$-chloropyridine $/ \mathrm{kg}$ body weight are presented in Table J-6. The blood concentration versus time data and estimated toxicokinetic parameters are shown in Figure J-3. Absorption of $o$-chloropyridine following gavage administration was rapid with a rapid decrease in the concentration of radiolabel ( $o$-chloropyridine equivalents) in blood. The data were best fit using a two-compartment model. The maximum concentration was reached within 30 minutes. The estimated distribution $\left(t_{1 / 2 \alpha}\right)$ and elimination $\left(t_{1 / 2}\right)$ half-lives, based on the total radioactivity, were 1.14 and 46 hours, respectively.

The blood concentrations of $o$-chloropyridine (ng/g blood) versus time in rats after a single intravenous injection of $1 \mathrm{mg} / \mathrm{kg}\left[{ }^{14} \mathrm{C}\right]-O$-chloropyridine are shown in Table $\mathrm{J}-7$ and Figure $\mathrm{J}-4$. The maximum concentration of $o$-chloropyridine in blood, $567 \mathrm{ng} / \mathrm{g}$, was measured at the earliest collection time ( 0.083 hours postdose). By 3 hours postdosing, the concentration of $o$-chloropyridine in the blood was too low to detect. A two-compartment model resulted in the best fits to the blood $o$-chloropyridine concentration-time data. (Data from one rat was poorly fitted by the simulation at the last two time points. For this reason, data for this rat was excluded from the calculation of the mean elimination half-life.) The distribution half-life $\left(\mathrm{t}_{1 / 2 \alpha}\right)$ of $o$-chloropyridine in the blood was a very rapid 0.103 hours. The elimination half-life $\left(\mathrm{t}_{1 / 2 \beta}\right)$ of $o$-chloropyridine in the blood was 1.04 hours (Figure J-4).

\section{J.4. Summary}

The objectives of these studies were to determine disposition and metabolism of $o$ chloropyridine in male rats after a single oral or injected (i.e., intravenous or intraperitoneal) dose and also to obtain basic toxicokinetic parameters following gavage and intravenous administration in male rats. Excretion and disposition studies were conducted in rats with oral 
gavage doses of $0.1 \mathrm{and} 10 \mathrm{mg} / \mathrm{kg}\left[{ }^{14} \mathrm{C}\right]$-o-chloropyridine. Results were comparable between the two doses indicating no dose-related difference in disposition. Absorption of $o$-chloropyridine following gavage administration was rapid with the maximum blood concentration reached within 30 minutes. Approximately $82 \%$ of the radiolabeled dose was recovered within 72 hours in both studies: $36 \%$ to $39 \%$ of the dose was excreted in the urine, $28 \%$ in feces, $10 \%$ to $13 \%$ was eliminated as $\mathrm{CO}_{2}$, and approximately $1 \%$ was excreted as volatile organics. The only tissues with appreciable tissue:blood ratios were the liver and the kidney. The estimated distribution $\left(\mathrm{t}_{1 / 2 \alpha}\right)$ and elimination $\left(\mathrm{t}_{1 / 2 \beta}\right)$ half-lives in blood, based on the total radioactivity, were 1.14 and 46 hours, respectively. Attempts were made to identify three urinary metabolites that comprised a large percentage of total radioactivity eluted in urine. Data from NMR spectroscopy and MS analyses indicated disubstituted pyridine structures. One of the metabolites contained mercapturic acid as one substituent and a hydroxyl group as the other, although it was uncertain as to whether the hydroxyl group was located at the 4 or 5 position on the pyridine ring. Data for the other two metabolites are consistent with hydroxylated $o$-chloropyridines, one of which was confirmed as 2-chloro-5-hydroxypyridine by comparison with a synthetic standard. Approximately 27\% of a single intraperitoneal dose of

$50 \mathrm{mg} / \mathrm{kg}$ was excreted in bile within 4 hours of dosing. Incubation of HPLC-fractionated bile with $\beta$-glucuronidase obtained from E. coli showed shifts in six of the eight metabolite fractions indicating the presence of several glucuronide metabolites. 
Table J-1. Disposition of Radioactivity in Male F344 Rats 72 Hours Following a Single Oral Gavage Dose of $\left.10 \mathrm{mg} / \mathrm{kg}^{14} \mathrm{C}\right]-o$-Chloropyridine ${ }^{\mathrm{a}}$

\begin{tabular}{cccccc}
\hline $\begin{array}{c}\text { End of } \\
\text { Collection } \\
\text { Period (Hours) }\end{array}$ & Urine & Feces & Exhaled CO2 & $\begin{array}{c}\text { Volatile } \\
\text { Organics }\end{array}$ & Total \\
\hline 6 & $16.3 \pm 3.8$ & $-^{\mathrm{b}}$ & $4.38 \pm 0.61$ & $0.954 \pm 0.298$ & $21.6 \pm 4.0$ \\
12 & $26.0 \pm 2.5$ & $3.43 \pm 4.7$ & $9.36 \pm 1.04$ & $1.06 \pm 0.31$ & $39.8 \pm 4.7$ \\
24 & $32.0 \pm 1.8$ & $25.0 \pm 2.3$ & $11.3 \pm 1.2$ & $1.11 \pm 0.33$ & $69.4 \pm 2.6$ \\
48 & $34.8 \pm 1.5$ & $28.0 \pm 2.6$ & $12.1 \pm 1.2$ & $1.13 \pm 0.33$ & $76.0 \pm 2.8$ \\
72 & $35.9 \pm 1.6^{\mathrm{c}}$ & $28.4 \pm 2.5$ & $12.5 \pm 1.2$ & $1.14 \pm 0.33$ & $78.0 \pm 3.0$ \\
\hline Disposition Summary & & & & & \\
Excreta & & & & & $78.0 \pm 3.0$ \\
Residual Carcass & & & & & $0.485 \pm 0.093$ \\
Tissues & & & & & $3.43 \pm 2.9$ \\
Total Dose Recovered & & & & & \\
\hline
\end{tabular}

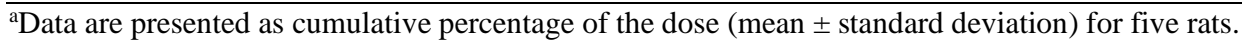

${ }^{b}$ No collection was scheduled for this time interval.

${ }^{\mathrm{c}}$ Includes cage rinse.

${ }^{\mathrm{d}}$ Percent dose recovered in the residual carcass less the percent dose measured in individual tissues: adipose, blood, muscle, and skin.

'Percent dose recovered in the following tissues: adipose, bladder, blood, brain, heart, kidney, liver, lung, muscle, skin, spleen, and testis. Also included were cecum, large and small intestine, and stomach, all with contents. 
Table J-2. Tissue Distribution of Radioactivity in Male F344 Rats 72 Hours Following a Single Oral Gavage Dose of $\left.10 \mathrm{mg} / \mathrm{kg}^{[14} \mathrm{C}\right]-o$-Chloropyridine ${ }^{\mathrm{a}}$

\begin{tabular}{|c|c|c|c|}
\hline Tissue & $\begin{array}{c}o \text {-Chloropyridine } \\
\text { Equivalents in Tissue } \\
\text { (ng-Eq/g Tissue) }\end{array}$ & Tissue:Blood Ratio & $\begin{array}{c}\text { Dose in Total Tissue } \\
(\%)^{\mathbf{b}}\end{array}$ \\
\hline Adipose $^{\mathrm{c}}$ & $140 \pm 84$ & $0.417 \pm 0.270$ & $0.0910 \pm 0.0545$ \\
\hline Bladder & $524 \pm 57$ & $1.53 \pm 0.25$ & $0.00185 \pm 0.00038$ \\
\hline Blood & $346 \pm 35$ & Unity & $0.167 \pm 0.018$ \\
\hline Brain & $101 \pm 10$ & $0.293 \pm 0.033$ & $0.00646 \pm 0.00134$ \\
\hline Cecum $^{\mathrm{d}}$ & NA & NA & $0.0826 \pm 0.0087$ \\
\hline Heart & $364 \pm 39$ & $1.06 \pm 0.16$ & $0.0110 \pm 0.0014$ \\
\hline Intestine, Large ${ }^{\mathrm{d}}$ & NA & NA & $0.0652 \pm 0.0144$ \\
\hline Intestine, Small ${ }^{\mathrm{d}}$ & NA & NA & $0.106 \pm 0.007$ \\
\hline Kidney & $2,500 \pm 309$ & $7.27 \pm 0.94$ & $0.176 \pm 0.007$ \\
\hline Liver & $3,660 \pm 471$ & $10.6 \pm 1.5$ & $1.47 \pm 0.15$ \\
\hline Lung & $442 \pm 34$ & $1.29 \pm 0.15$ & $0.0158 \pm 0.0014$ \\
\hline Muscle $^{\mathrm{c}}$ & $153 \pm 18$ & $0.445 \pm 0.056$ & $0.682 \pm 0.083$ \\
\hline Skin, Ear & $291 \pm 34$ & $0.845 \pm 0.110$ & $0.459 \pm 0.055$ \\
\hline Spleen & $506 \pm 35$ & $1.48 \pm 0.19$ & $0.0108 \pm 0.0014$ \\
\hline Stomach $^{\mathrm{d}}$ & NA & NA & $0.0636 \pm 0.0072$ \\
\hline Testis & $139 \pm 7$ & $0.404 \pm 0.039$ & $0.0158 \pm 0.0010$ \\
\hline Carcass ${ }^{\mathrm{e}}$ & NA & NA & $0.485 \pm 0.093$ \\
\hline
\end{tabular}

NA = Not applicable.

${ }^{\text {aD }}$ Data are presented as mean \pm standard deviation for five rats.

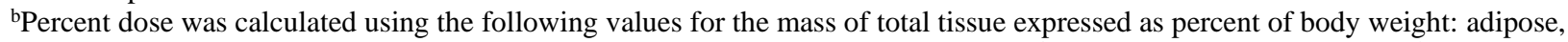
7.0\%; blood, 5.2\%; muscle, 48.0\%; and skin, $17 \%$ (taken from Donaldson ${ }^{67}$; Adolph ${ }^{68}$; Supplee et al. ${ }^{69}$; Caster et al. ${ }^{70}$; Bischoff et al. ${ }^{71}$; and Lutz et al. ${ }^{72}$ ).

${ }^{\mathrm{c}}$ Adipose and muscle values are averaged results for two sampling locations.

${ }^{\mathrm{d}}$ Includes contents.

${ }^{\mathrm{e}}$ Carcass value is based on the residual digested carcass after the removal of the listed tissues (i.e., percent dose measured in adipose, blood, muscle, and skin was subtracted from the total percent dose measured in the carcass). 
Table J-3. Disposition of Radioactivity in Male F344 Rats 72 Hours Following a Single Oral Gavage

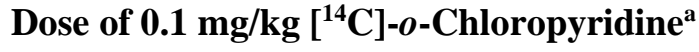

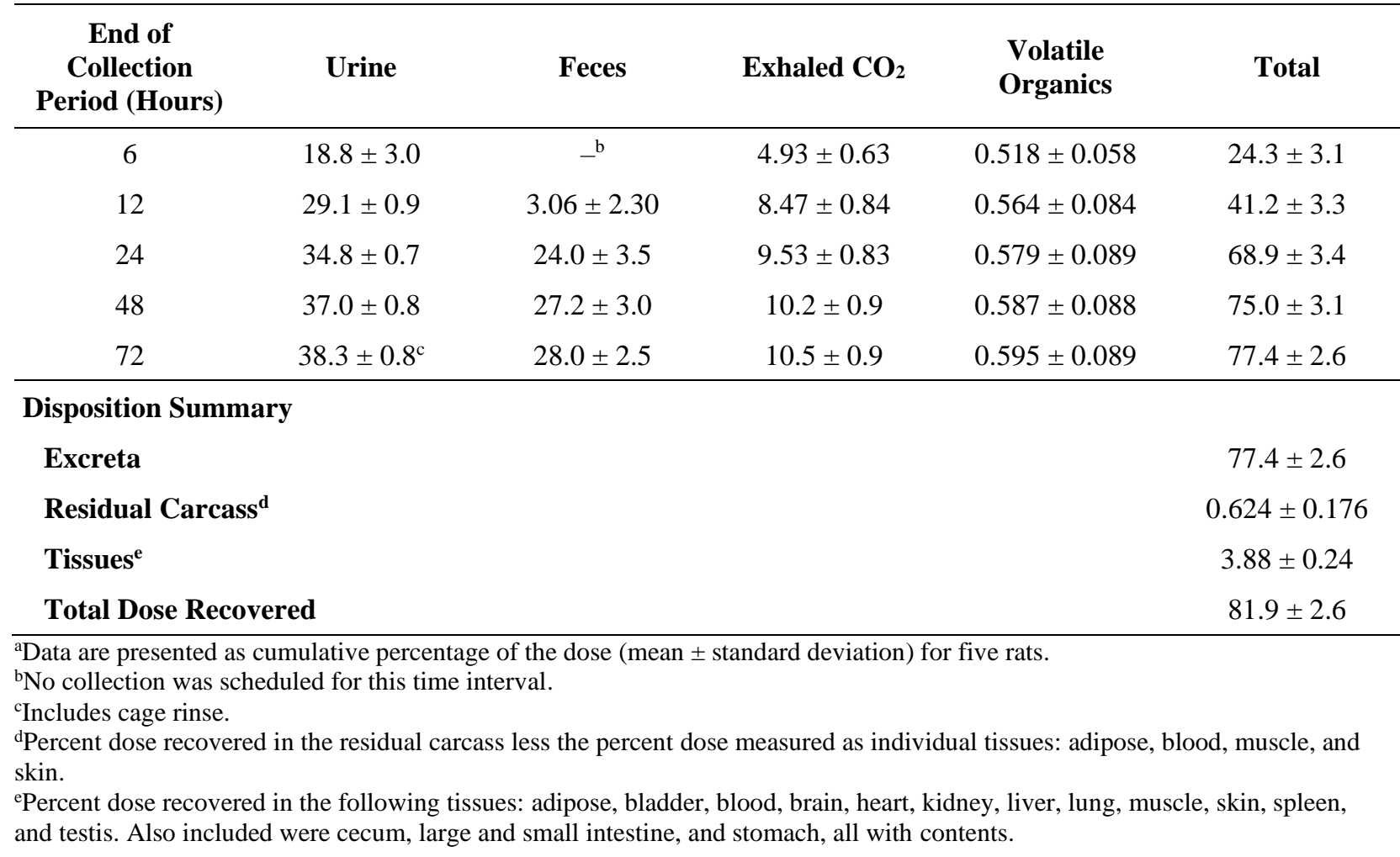


Table J-4. Tissue Distribution of Radioactivity in Male F344 Rats 72 Hours Following a Single Oral Gavage Dose of $\left.0.1 \mathrm{mg} / \mathrm{kg}^{[14} \mathrm{C}\right]-o$-Chloropyridine ${ }^{\mathrm{a}}$

\begin{tabular}{|c|c|c|c|}
\hline Tissue & $\begin{array}{c}o \text {-Chloropyridine } \\
\text { Equivalents in Tissue } \\
\text { (ng-Eq/g Tissue) }\end{array}$ & Tissue:Blood Ratio & $\begin{array}{c}\text { Dose in Total Tissue } \\
(\%)^{\mathbf{b}}\end{array}$ \\
\hline Adipose $^{\mathrm{c}}$ & $1.43 \pm 0.44$ & $0.382 \pm 0.122$ & $0.0867 \pm 0.0253$ \\
\hline Bladder & $7.69 \pm 1.46$ & $7.69 \pm 1.46$ & $0.00221 \pm 0.00055$ \\
\hline Blood & $3.76 \pm 0.07$ & Unity & $0.171 \pm 0.007$ \\
\hline Brain & $1.28 \pm 0.07$ & $0.341 \pm 0.018$ & $0.00854 \pm 0.00076$ \\
\hline Cecum $^{\mathrm{d}}$ & NA & NA & $0.153 \pm 0.040$ \\
\hline Heart & $4.69 \pm 0.23$ & $1.25 \pm 0.069$ & $0.0147 \pm 0.0010$ \\
\hline Intestine, Large ${ }^{\mathrm{d}}$ & NA & NA & $0.0416 \pm 0.0095$ \\
\hline Intestine, Small ${ }^{\mathrm{d}}$ & NA & NA & $0.125 \pm 0.017$ \\
\hline Kidney & $27.1 \pm 3.03$ & $7.22 \pm 0.93$ & $0.185 \pm 0.013$ \\
\hline Liver & $49.0 \pm 4.8$ & $13.0 \pm 1.27$ & $1.44 \pm 0.06$ \\
\hline Lung & $5.68 \pm 0.276$ & $1.51 \pm 0.086$ & $0.0214 \pm 0.0034$ \\
\hline Muscle $^{c}$ & $2.58 \pm 0.37$ & $0.687 \pm 0.109$ & $1.08 \pm 0.15$ \\
\hline Skin, Ear & $3.16 \pm 0.40$ & $0.839 \pm 0.105$ & $0.468 \pm 0.066$ \\
\hline Spleen & $6.33 \pm 0.33$ & $1.68 \pm 0.10$ & $0.0118 \pm 0.0001$ \\
\hline Stomach $^{\mathrm{d}}$ & NA & NA & $0.0578 \pm 0.0157$ \\
\hline Testis & $1.64 \pm 0.06$ & $0.437 \pm 0.015$ & $0.0172 \pm 0.0010$ \\
\hline Carcass $^{\mathrm{e}}$ & NA & NA & $2.42 \pm 0.07$ \\
\hline
\end{tabular}

NA = Not applicable.

${ }^{\text {a}}$ Data are presented as mean \pm standard deviation for five rats.

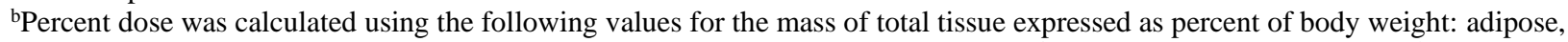
7.0\%; blood, 5.2\%; muscle, 48.0\%; and skin, $17 \%$ (taken from Donaldson ${ }^{67}$; Adolph $^{68}$; Supplee et al. ${ }^{69}$; Caster et al. ${ }^{70}$; Bischoff et al. ${ }^{71}$; and Lutz et al. ${ }^{72}$ ).

${ }^{\mathrm{c}}$ Adipose and muscle values are averaged results for two sampling locations.

${ }^{\mathrm{d}}$ Includes contents.

${ }^{\mathrm{e}}$ Carcass value is based on the residual digested carcass after the removal of the listed tissues (i.e., percent dose measured in adipose, blood, muscle, and skin was subtracted from the total percent dose measured in the carcass). 
Table J-5. Excretion of Radioactivity in Bile in Male F344 Rats Following a Single Intraperitoneal

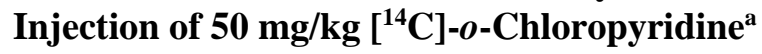

\begin{tabular}{cc}
\hline End of Collection Period (hours) & Bile \\
\hline 0.5 & $1.99 \pm 0.69$ \\
1.0 & $6.10 \pm 2.59$ \\
1.5 & $9.86 \pm 4.55$ \\
2.0 & $13.5 \pm 5.7$ \\
2.5 & $16.6 \pm 6.3$ \\
3.0 & $20.8 \pm 7.9$ \\
3.5 & $23.6 \pm 8.2$ \\
4.0 & $26.9 \pm 8.7$ \\
\hline
\end{tabular}

${ }^{\mathrm{a}}$ Data are presented as cumulative percentage of the dose (mean \pm standard deviation) for five rats.

Table J-6. Total Radioactive Equivalents in Blood Over Time in Male F344 Rats Following a Single Oral Gavage Dose of $10 \mathrm{mg} / \mathrm{kg}\left[{ }^{14} \mathrm{C}\right]-o$-Chloropyridine ${ }^{\mathrm{a}}$

\begin{tabular}{ccc}
\hline $\begin{array}{c}\text { Time After Dosing } \\
\text { (hours) }\end{array}$ & $\begin{array}{c}\boldsymbol{o} \text {-Chloropyridine Equivalents in } \\
\text { Blood } \\
\text { (ng-Eq/g Blood) }\end{array}$ & $\begin{array}{c}\text { Dose in Total Blood } \\
(\boldsymbol{\%})\end{array}$ \\
\hline 0.083 & $2,062 \pm 316$ & $1.07 \pm 0.16$ \\
0.25 & $3,376 \pm 738$ & $1.75 \pm 0.38$ \\
0.50 & $3,807 \pm 462$ & $1.97 \pm 0.24$ \\
1 & $3,278 \pm 570$ & $1.70 \pm 0.29$ \\
1.5 & $2,687 \pm 243$ & $1.39 \pm 0.12$ \\
2 & $2,192 \pm 305$ & $1.14 \pm 0.16$ \\
3 & $1,573 \pm 166$ & $0.814 \pm 0.086$ \\
4 & $1,224 \pm 111$ & $0.634 \pm 0.057$ \\
5 & $1,153 \pm 78$ & $0.597 \pm 0.040$ \\
6 & $1,092 \pm 73$ & $0.565 \pm 0.038$ \\
12 & $917 \pm 102$ & $0.475 \pm 0.053$ \\
24 & $724 \pm 87$ & $0.375 \pm 0.045$ \\
$48^{\mathrm{b}}$ & $475 \pm 58$ & $0.246 \pm 0.030$ \\
$72^{\mathrm{c}}$ & $353 \pm 23$ & $0.183 \pm 0.012$ \\
\hline
\end{tabular}

${ }^{\mathrm{a}}$ Data are presented as mean \pm standard deviation for six rats. 
Table J-7. Concentration of $o$-Chloropyridine and Total Radioactive Equivalents in Blood Over Time in Male F344 Rats Following a Single Intravenous Injectionof $1 \mathrm{mg} / \mathrm{kg}\left[{ }^{14} \mathrm{C}\right]-\mathrm{o}$ Chloropyridine $^{\mathrm{a}}$

\begin{tabular}{cccc}
\hline $\begin{array}{c}\text { Time After Dosing } \\
\text { (Hours) }\end{array}$ & $\begin{array}{c}\text { o-Chloropyridine } \\
\text { (ng/g Blood) }\end{array}$ & $\begin{array}{c}\text { Radiolabel } \\
\text { o-Chloropyridine } \\
\text { Equivalents } \\
\text { (ng-Eq/g Blood) }\end{array}$ & $\begin{array}{c}\text { Radiolabel Dose in Total } \\
\text { Blood (\%) }\end{array}$ \\
\hline 0.083 & $567 \pm 23$ & $632 \pm 146$ & $4.09 \pm 0.99$ \\
0.17 & $437 \pm 31$ & $422 \pm 39$ & $2.76 \pm 0.27$ \\
0.25 & $360 \pm 31$ & $356 \pm 5$ & $2.30 \pm 0.06$ \\
0.50 & $220 \pm 20$ & $286 \pm 35$ & $1.85 \pm 0.26$ \\
1 & $123 \pm 10$ & $209 \pm 37$ & $1.35 \pm 0.24$ \\
1.5 & $100 \pm 43$ & $138 \pm 11$ & $0.893 \pm 0.075$ \\
2 & $73.2 \pm 53.4$ & $100 \pm 9$ & $0.646 \pm 0.069$ \\
3 & $\mathrm{ND}$ & $74.9 \pm 6.1$ & $0.484 \pm 0.041$ \\
4 & $\mathrm{ND}$ & $74.9 \pm 4.8$ & $0.483 \pm 0.025$ \\
\hline
\end{tabular}

$\mathrm{ND}=$ Not determined.

${ }^{\text {aD }}$ ata are presented as mean \pm standard deviation for four rats. 


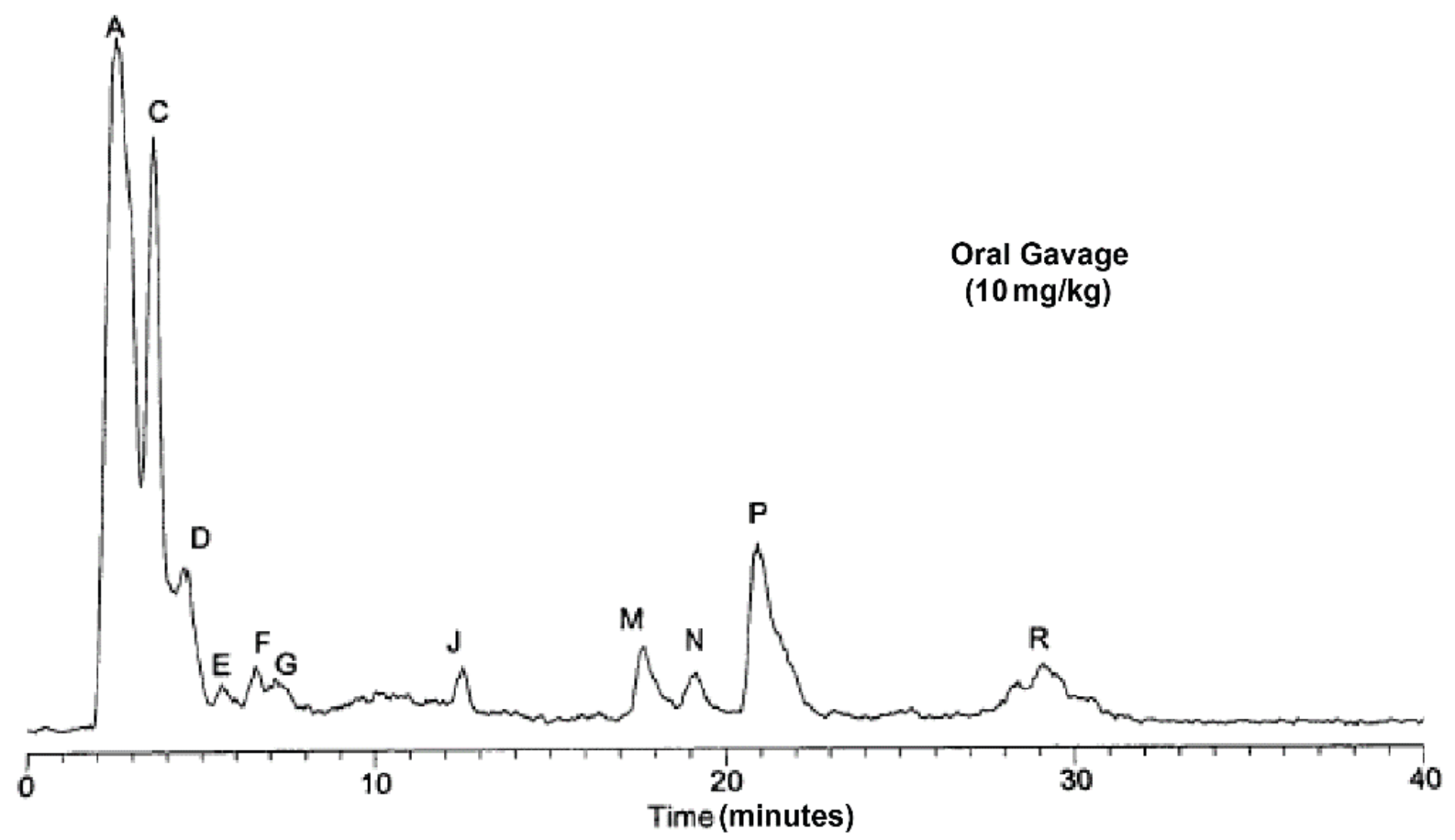

Figure J-1. $\left[{ }^{14} \mathrm{C}\right]$-Labeled Urinary Metabolites in Male F344 Rats Following a Single Oral Gavage Dose of $10 \mathrm{mg} / \mathrm{kg}\left[{ }^{14} \mathrm{C}\right]-o$-Chloropyridine

Metabolites were determined in a composite ( 0 to 24 hours) urine sample from one rat. 
$o$-Chloropyridine, NTP TOX 83
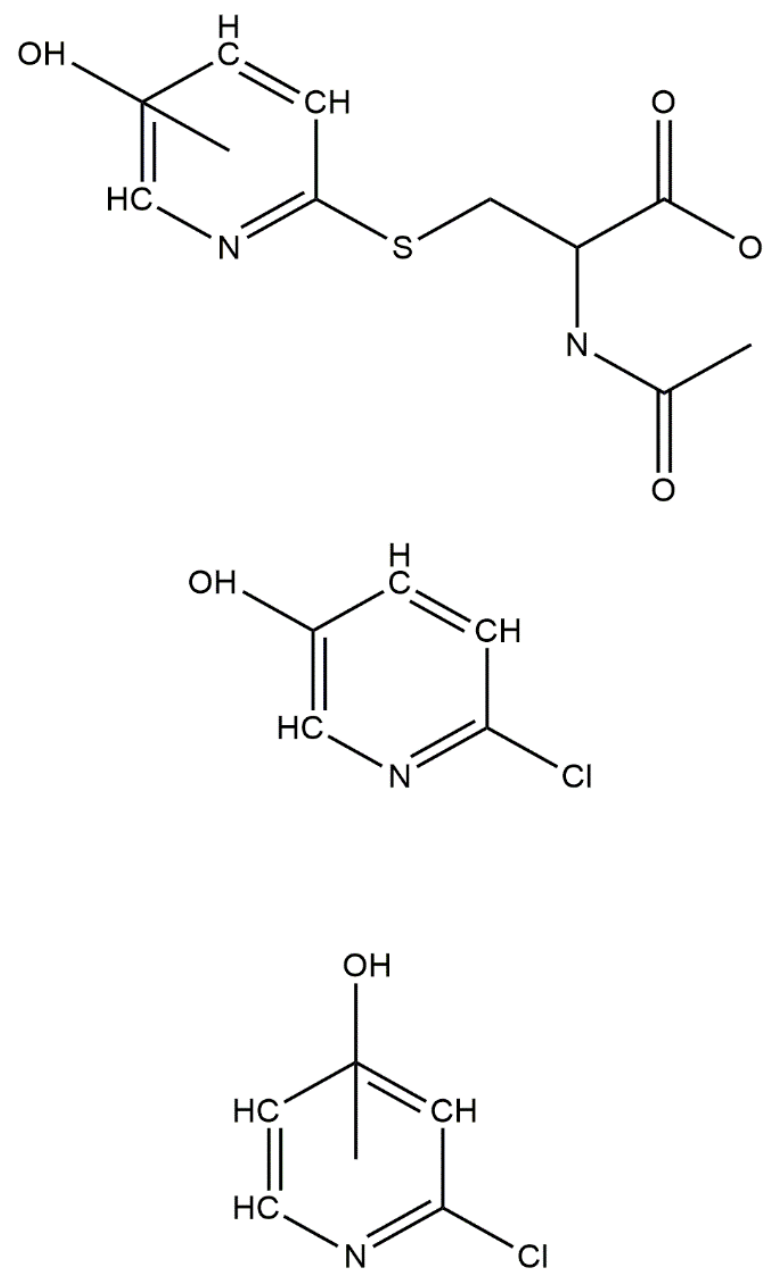

Figure J-2. Disubstituted Urinary Metabolites of $o$-Chloropyridine in Male F344 Rats 


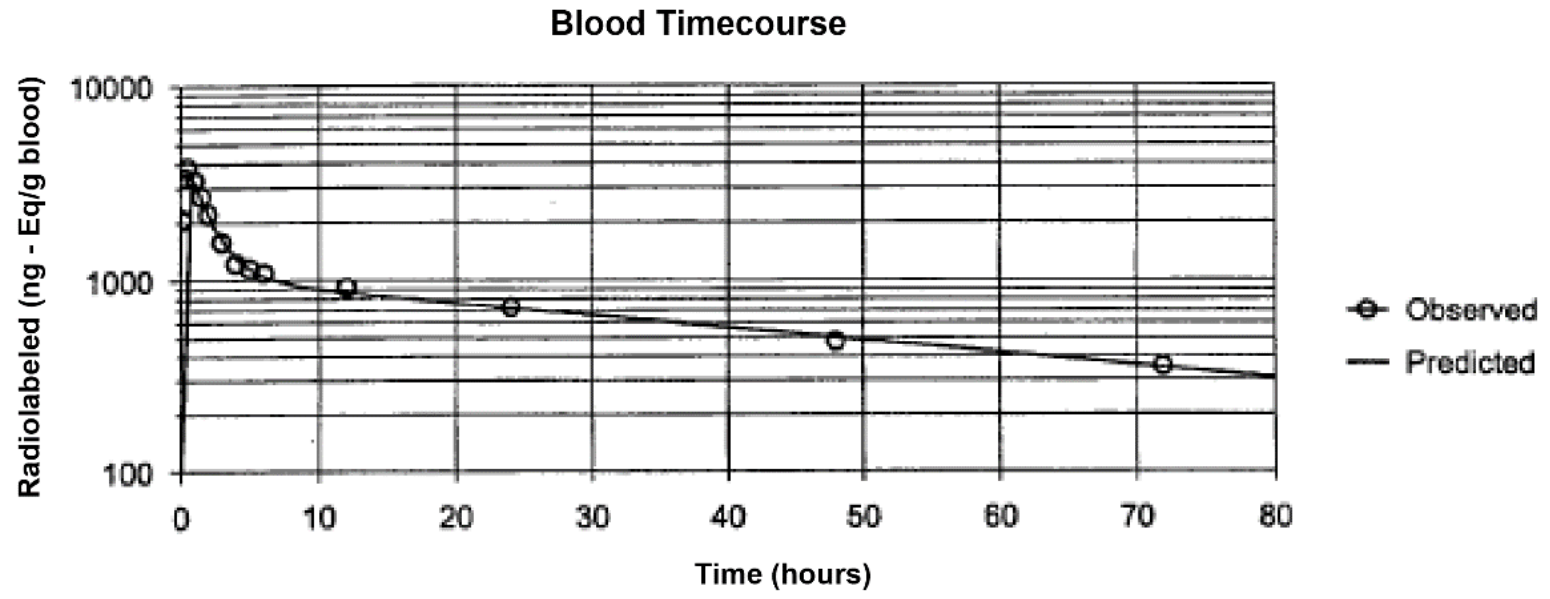

Toxicokinetic Parameters

\begin{tabular}{lccccc}
\hline Parameter & Estimate & Std Error & CV\% & UnivarCl Lower & UnivarCl Upper \\
\hline A & 2129 & 110.1 & 5.17 & 1880 & 2378 \\
B & 546.5 & 51.16 & 9.36 & 430.7 & 662.2 \\
K01 & 6.71 & 0.540 & 8.05 & 5.48 & 7.93 \\
Alpha & 0.607 & 0.0613 & 10.1 & 0.468 & 0.746 \\
Beta & 0.0151 & 0.00354 & 23.5 & 0.00706 & 0.0231 \\
\hline \hline
\end{tabular}

Distribution of half-life of radiolabel: $\quad t_{1 / 2}=0.693$ /alpha $=1.14$ hours

Elimination of half-life of radiolabel: $t_{1 / 2}=0.693 /$ beta $=46.0$ hours

Figure J-3. Blood Timecourse and Toxicokinetic Parameter Estimates of $o$-Chloropyridine in Male F344 Rats Following a Single Oral Gavage Dose of $10 \mathrm{mg} / \mathrm{kg}\left[{ }^{14} \mathrm{C}\right]-o$-Chloropyridine

Data were fitted using a two-compartment model. Constants A and B are intercepts on the y axis for each exponential segment of the curve and $\beta$ and $\alpha$ are the elimination and distribution rate constants, respectively. 


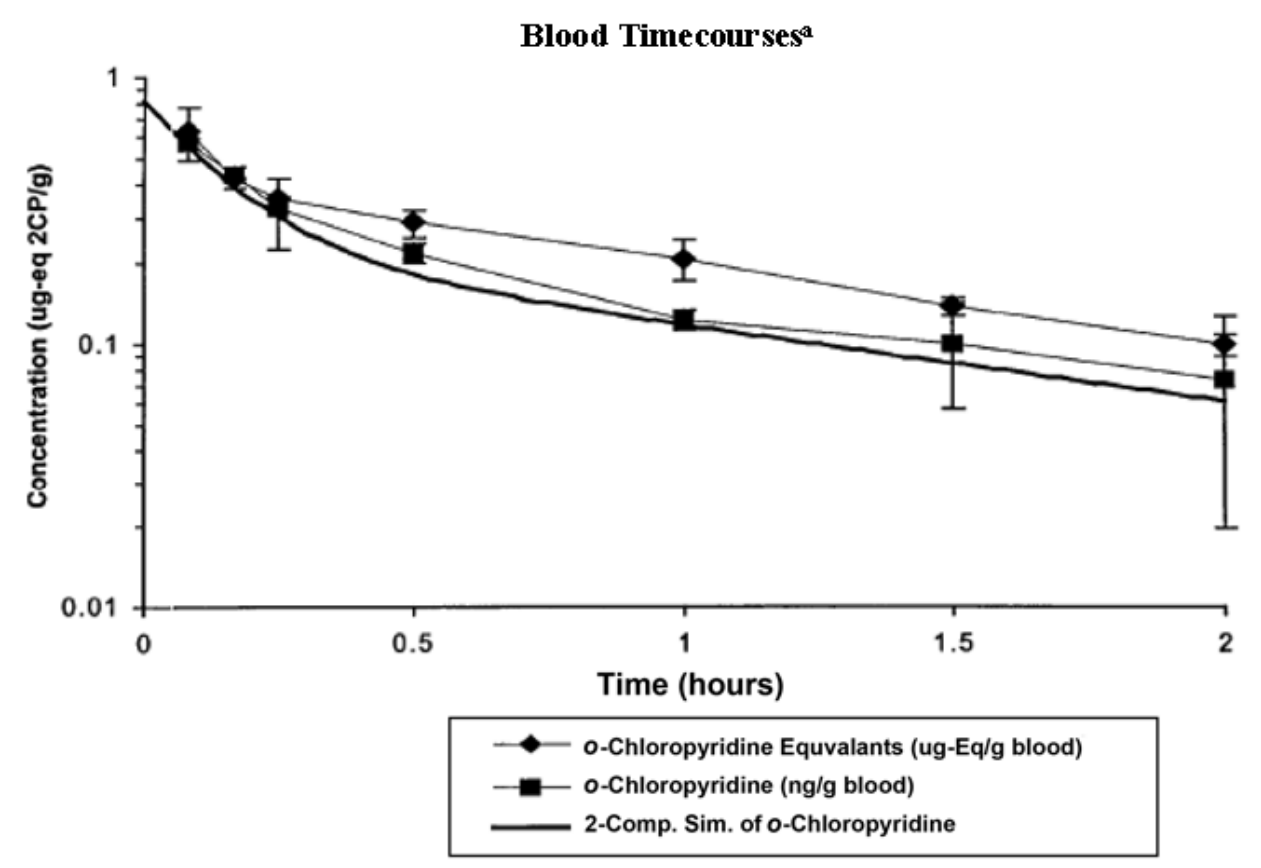

Toxicokinetic Parameters

Values from 2-Compartment Simulation of $a$-Chloropyridine in Blood ${ }^{\mathbf{b}}$

\begin{tabular}{lccc}
\hline \multicolumn{1}{c}{ Parameter } & Mean & $\begin{array}{c}\text { Standard } \\
\text { Deviation }\end{array}$ & CV\% \\
\hline Volume of distribution (mL/kg) & 982 & 221 & 22.5 \\
Alpha (hour-1) & 6.71 & 3.42 & 50.9 \\
Beta (hour-1) & 0.669 & 0.028 & 4.22 \\
Distribution half-life (hours) & 0.103 & $\mathrm{NA}$ & $\mathrm{NA}$ \\
Elimination half-life (hours) & 1.04 & $\mathrm{NA}$ & $\mathrm{NA}$ \\
Clearance (mL/min/kg) & 29.8 & 2.5 & 8.39 \\
\hline
\end{tabular}

NA=Not app licable. Samples beyond 2 hours contained insufficient radioactivity for analysis. avalues are mean \pm standard deviation for four rats.

bValues are mean \pm standard deviation for three rats; rat number 5 was dropped from the simulation due to variability in the concentration of $o$-chloropyridine present at the latest two time points for this animal; the simulation was skewed if rat number 5 was included.

Figure J-4. Blood Timecourse and Toxicokinetic Parameter Estimates for o-Chloropyridine and Radiolabel (o-Chloropyridine Equivalents) in Blood of Male F344 Rats Following a Single Intravenous Injection of $1 \mathrm{mg} / \mathrm{kg}\left[{ }^{14} \mathrm{C}\right]-o$-Chloropyridine 


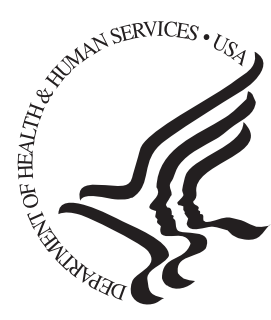

\section{National Toxicology Program}

\section{NTP Central Data Management, MD EC-03}

National Institute of Environmental Health Sciences

P.O. Box 12233

Research Triangle Park, NC 27709

http://ntp.niehs.nih.gov 